\title{
Three-Component Reaction for the Synthesis of Highly Functionalized Propargyl Ethers
}

\author{
Guillaume Pisella, Alec Gagnebin and Jérôme Waser*
}

\begin{abstract}
Multicomponent reactions provide efficient means to access molecular complexity. Herein, we report a copper-catalyzed three-component reaction of diazo compounds, alcohols and ethynyl benziodoxole $(E B X)$ reagents for the synthesis of propargyl ethers. Extensive variations of the three partners of the reaction is possible, leading to highly functionalized and structurally diverse products under mild conditions. Alkynylation of a copper ylide intermediate is postulated as key step for this transformation.
\end{abstract}

Multicomponent reactions are ideally suited for a fast entry into molecular complexity. ${ }^{[1]}$ It is therefore not surprising that classical multicomponent reactions such as the Ugi or the Passerini reactions have had a major impact on both synthetic and medicinal chemistry. ${ }^{[2]}$ Recently, diazo compounds have emerged as ideal partners in multicomponent reactions, with or without the help of transition metal catalysts. ${ }^{[3]}$ In particular, transient ylide intermediates generated from the insertion of protic nucleophiles (alcohols, amines, thiols and aromatic compounds) into metalcarbenes generated from diazo compounds have been intercepted with various electrophiles (carbonyl compounds, imines, Michael acceptors, azodicarboxylates, electrophilic halogen sources,...) to simultaneously generate two new bonds (Scheme 1A). ${ }^{[4]}$ To further develop the use of this strategy in multicomponent reactions, it is now important to extend the scope of compatible nucleophiles/electrophiles for achieving a higher molecular diversity.

In this context, hypervalent iodine reagents in general[ ${ }^{[5]}$ and cyclic derivatives in particular ${ }^{[6]}$ have demonstrated their versatility for the Umpolung of the reactivity of functional groups, giving access to a broad range of non-classical electrophiles. However, their use in multi-component reactions involving diazo compounds is not yet well established. In the absence of a metal-catalyst, Murphy and co-workers have first reported dihalogenation reactions. ${ }^{[7]}$ More recent examples involve acetoxyaminoalkylation ${ }^{[8]}$ and azidoaminoalkylation. ${ }^{[9]}$ The first transformations involving metal carbene intermediates were reported independently by our group ${ }^{[10]}$ and Szabo and co-workers. ${ }^{[11]}$ We developed a coppercatalyzed oxy-alkynylation using ethynylbenziodoxolone (EBX) reagents, whereas Szabo and co-workers successfully implemented a rhodium-catalyzed oxy-fluoro/trifluoromethylation with benziodoxole reagents (Scheme 1B). These two transformations constitute efficient multi-bond forming reactions combining hypervalent iodine reagents and diazo compounds and most probably proceed via ylide intermediates generated from metal carbenes. Nevertheless, they remain strongly limited in the diversity of structures accessible: our work allowed exclusively the introduction of iodo-benzoate derivatives as nucleophilic

[] G. Pisella, A. Gagnebin, Prof. Dr. J. Waser

Laboratory of Catalysis and Organic Synthesis, Ecole Polytechnique

Fédérale de Lausanne, EPFL, SB ISIC LCSO, BCH 4306

1015 Lausanne (Switzerland)

Email: jerome.waser@epfl.ch

Homepage : http://lcso.epfl.ch/

Supporting information for this article is given via a link at the end of the document. partners in the three-component reaction, whereas the work of Szabo-and co-workers was limited to fluoride or trifluoromethyl as one of the partners. Therefore, there is an urgent need for more general three-component reactions of diazo compounds, nucleophiles and hypervalent iodine reagents, allowing for extensive variation of the three partners with diverse functional groups in order to maximize the structural diversity of the products.

Herein, we report the copper-catalyzed three-component reaction of diazo compounds, ethynylbenziodoxoles (EBXs) and alcohols, which overcomes this limitation (Scheme 1C). The three components of the reaction can be extensively varied, leading to a broad range of important propargylic ether building blocks with high structural diversity. ${ }^{[12]}$ In particular, primary, secondary and tertiary alcohols, as well as a broad range of functional groups (including alkene, alkyne, fluoro, chloro, bromo, ether, ester, ketone, carbamate, imide, cyano, boronic ester and heterocyclic groups) were well tolerated, which would be difficult to achieve using traditional etherification methods under strongly basic or acidic conditions. The transformation can be performed using simple copper salts as catalyst, and does not require the use of one of the partners in large excess.

A) Multi-components reactions from diazo compounds via trapping of ylide intermediates

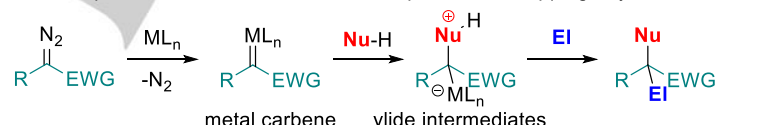

$$
\begin{aligned}
& \text { metal carbene ylide intermediates } \\
& \begin{array}{rl:c}
\mathrm{Nu}-\mathrm{H}=\mathrm{R}^{1} \mathrm{OH}, \mathrm{R}^{1}{ }_{2} \mathrm{NH}, \mathrm{R}^{1} \mathrm{SH}, \mathrm{ArH} & \mathrm{El}=\begin{array}{c}
\text { carbonyls, imines, Michael acceptors } \\
\text { azodicarboxylates, } \mathrm{X}^{+}
\end{array}
\end{array}
\end{aligned}
$$

B) Reactions of diazo with hypervalent iodine reagents via metal carbene intermediates

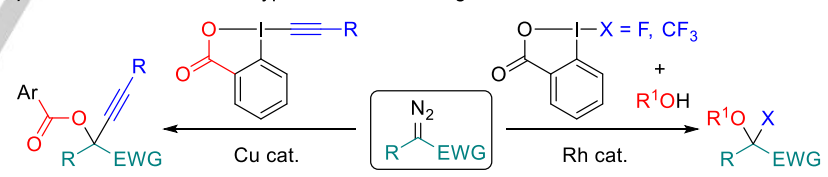
$\mathrm{Ar}=2$-iodobenzoyl Oxyalkynylation ${ }^{[10]}$ Oxytrifluoromethylation ${ }^{[11]}$ and Oxyfluorination

C) This work: Three-component reactions of alcohols, diazo and EBX reagents

$$
\text { 等 }
$$

Scheme 1. Multi-component reactions of diazo compounds involving ylide intermediates and new disconnections enabled by hypervalent iodine reagents.

We first investigated the three-component reaction of TIPS-EBX (1), ethyl diazoacetate (3a) and ethanol (4a) (Table 1). In our previous work on the two-component process, diimine an bisoxazoline ligands on the copper catalyst led to best results. ${ }^{[10]}$ Using $\mathrm{Cu}(\mathrm{MeCN})_{4} \mathrm{BF}_{4}$ as catalyst, diimine $\mathbf{8}$ or tert-butyl bisoxazoline (tBu-BOX (9)) as ligands and ethanol as solvent, the desired product 5 a could be obtained in $50 \%$ and $63 \%$ yield, respectively (entries 1 and 2). However, despite using ethanol as solvent, a significant amount (30-32\%) of the two-component product 7a was still obtained. Furthermore, when only 10 
equivalents of ethanol were used, the yield of 6 a dropped to $22 \%$ (entry 3), showing that these conditions would not be useful to develop a general three-component reaction.

Table 1. Optimization of the three-component reaction

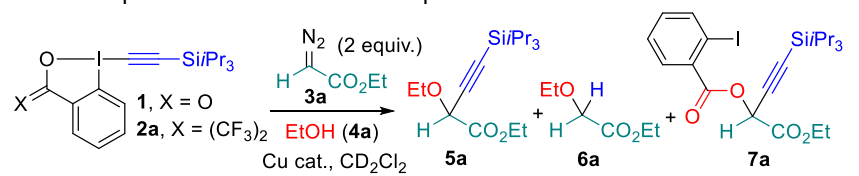

\begin{tabular}{|c|c|c|c|c|}
\hline Entry & EBX & $\begin{array}{c}\text { Equiv } \\
\text { EtOH (4a) }\end{array}$ & Catalyst/Ligand(additive) & $\begin{array}{c}\text { Yield } 5 a / 6 a / 7 a \\
{[\%]^{[a]}}\end{array}$ \\
\hline
\end{tabular}

\begin{tabular}{|c|c|c|c|c|}
\hline 1 & 1 & as solvent & $4 \mathrm{~mol} \% \mathrm{Cu}(\mathrm{MeCN})_{4} \mathrm{BF}_{4} / 8$ & $50 /$ n.d./32 \\
\hline 2 & 1 & as solvent & $4 \mathrm{~mol} \% \mathrm{Cu}(\mathrm{MeCN})_{4} \mathrm{BF}_{4} / \mathbf{9}$ & $63 /$ n.d. $/ 30$ \\
\hline 3 & 1 & 10 & $4 \mathrm{~mol} \% \mathrm{Cu}(\mathrm{MeCN})_{4} \mathrm{BF}_{4} / 9$ & 22/n.d./52 \\
\hline 4 & $2 a$ & as solvent & $4 \mathrm{~mol} \% \mathrm{Cu}(\mathrm{MeCN})_{4} \mathrm{BF}_{4} / \mathbf{9}$ & 62/n.d./- \\
\hline 5 & $2 a$ & as solvent & $4 \mathrm{~mol} \% \mathrm{Cu}(\mathrm{MeCN})_{4} \mathrm{BF}_{4}$ & $100 / 48 /-$ \\
\hline 6 & $2 a$ & 10 & $4 \mathrm{~mol} \% \mathrm{Cu}(\mathrm{MeCN})_{4} \mathrm{BF}_{4}$ & $100 / 47 /-$ \\
\hline 7 & $2 a$ & 4 & $4 \mathrm{~mol} \% \mathrm{Cu}(\mathrm{MeCN})_{4} \mathrm{BF}_{4}$ & $80 / 49 /-$ \\
\hline 8 & $2 a$ & 2 & $4 \mathrm{~mol} \% \mathrm{Cu}(\mathrm{MeCN})_{4} \mathrm{BF}_{4}$ & $53 / 51 /-$ \\
\hline 9 & $2 a$ & 2 & $10 \mathrm{~mol} \% \mathrm{Cu}(\mathrm{MeCN})_{4} \mathrm{BF}_{4}$ & $62 / 38 /-$ \\
\hline 10 & $2 a$ & 2 & $10 \mathrm{~mol} \% \mathrm{Cu}(\mathrm{MeCN})_{4} \mathrm{PF}_{6}$ & $74 /$ \\
\hline 11 & $2 a$ & 2 & 10 mol\% CuOTf-toluene & $60 / 33 /-$ \\
\hline 12 & $2 a$ & 2 & $10 \mathrm{~mol}^{2} \mathrm{CuOTf}_{2}$ & \\
\hline 13 & $2 a$ & 2 & $10 \mathrm{~mol} \% \mathrm{CuBr}$ & \\
\hline 14 & $2 a$ & 2 & $10 \mathrm{~mol}^{2} \mathrm{CuCl}_{2}$ & \\
\hline 15 & $2 a$ & 2 & $10 \mathrm{~mol} \% \mathrm{CuTC}$ & $10 / 40$ \\
\hline $16^{[\mathrm{b}]}$ & $2 a$ & 2 & $10 \mathrm{~mol} \% \mathrm{Cu}(\mathrm{MeCN})_{4} \mathrm{BF}_{4} / \mathrm{NaHCO}_{3}$ & $50 / 36 /-$ \\
\hline 17 & $2 a$ & 4 & $10 \mathrm{~mol} \% \mathrm{Cu}(\mathrm{MeCN})_{4} \mathrm{PF}_{6}$ & $94 / 1$ \\
\hline $18^{[\mathrm{c}]}$ & $2 a$ & 1 & $10 \mathrm{~mol} \% \mathrm{Cu}(\mathrm{MeCN})_{4} \mathrm{PF}_{6}$ & $37 / 17 /-$ \\
\hline
\end{tabular}

[a] Determined by ${ }^{1} \mathrm{H}$ NMR analysis of the crude reaction mixture. The hypervalent iodine reagent $\mathbf{1 / 2} \mathbf{a}$ and the diazo compound $\mathbf{3 a}$ are used as limiting reagents to calculate the yield of $5 a / 7 a$ and $6 a$ respectively. Reactions were run on a $0.08 \mathrm{mmol}$ scale. n.d. $=$ not determined. ${ }^{[b]}$ With 2 equivalents of sodium bicarbonate. ${ }^{[c]}$ One equivalent of diazo compound 3 a was used.

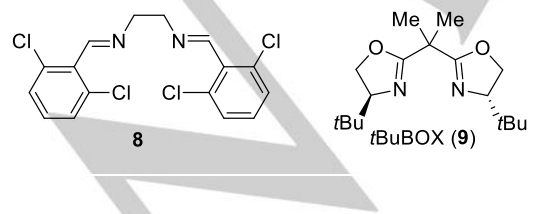

We therefore decided to modify the hypervalent iodine reagent. We turned to hexafluoroisopropanol derivative $2 \mathbf{2},{ }^{[13]}$ expecting a lower nucleophilicity of the oxygen atom. Indeed, in this case only the three-component product 5 a was obtained in $62 \%$ yield (entry 4). An enhanced reactivity was observed in absence of the BOX ligand, resulting in quantitative formation of product $5 \mathbf{a}$ together with $\mathrm{O}-\mathrm{H}$ insertion product $6 \mathrm{a}$ (entry 5). Gratifyingly, the same result was obtained when only 10 equivalents of ethanol (4a) were used (entry 6). The yield of 5 a decreased to $80 \%$ and $53 \%$ respectively with 4 and 2 equivalent of ethanol (4a) (entries 7 and $8)$. Using a higher catalyst loading, 5 a could be still obtained in $62 \%$ with only 2 equivalents of ethanol (4a) (entry 9 ). At this point, different copper salts were examined. Complexes with noncoordinating counterions performed better (entries 10-12) than copper halogenides (entries 13 and 14) or thiophenecarboxylate (TC, entry 15). ${ }^{[14]}$ The best result was obtained with $\mathrm{PF}_{6}{ }^{-}$as counterion, giving $5 \mathrm{a}$ in $74 \%$ yield, with only $18 \%$ of $\mathrm{O}-\mathrm{H}$ insertion product 6a formed (entry 10). We then wondered if basic condition may slow down the formation of $6 \mathbf{a}$. However, a lower yield was observed in presence of sodium hydrogen carbonate (entry 16) and the reaction completely stopped in presence of other bases such as carbonate, acetate or hydroxide salts (result not shown, see Supporting Information). For cheap alcohols a larger excess is reasonable, and 5 a could be obtained in $94 \%$ yield with 4 equivalents of ethanol (4a) (entry 17). Unfortunately, the formation of the alcohol insertion side product cannot be fully suppressed and it was observed for most transformations described in this study. Nevertheless, 5 a was still formed in $37 \%$ yield when EBX $\mathbf{2 a}$, diazo compound $\mathbf{3} \mathbf{a}$ and ethanol (4a) were used in equimolar amounts (entry 18).

With optimized conditions in hand, we started to investigate the scope of the three-component reaction. First, we employed a variety of alcohols with EBX $\mathbf{2 a}$ and ethyl diazo acetate $\mathbf{3 a}$ as the two other partners (Scheme 2A). On $0.3 \mathrm{mmol}$ scale, propargylic ether $5 \mathbf{a}$ was isolated in $93 \%$ yield. ${ }^{[15]}$ Benzyl alcohol provided the corresponding product $\mathbf{5 b}$ in a slightly diminished yield. Introducing a bromo substituent in the para position gave $\mathbf{5 c}$ in $73 \%$ yield. A heteroaromatic ring was also tolerated providing product $5 \mathbf{d}$ from furfuryl alcohol in $63 \%$ yield. We also examined secondary alcohols. Cyclohexanol was well tolerated and furnished the corresponding product 5 e in $90 \%$ yield. We were pleased to see that the more electron-poor 1,3-difluoro-2propanol gave the desired product $\mathbf{5 f}$ in good yield. Sterically hindered tert-butanol still reacted remarkably well to give the corresponding three-component product $\mathbf{5 g}$ in $69 \%$ yield. Such tert-butyl ethers represent a synthetic challenge and are classically accessed by strong acid-catalyzed addition of isobutene to alcohols. ${ }^{[16]}$ In the case of an allyl substituent, no reaction was observed at room temperature, but at $40{ }^{\circ} \mathrm{C} 5 \mathrm{~h}$ was obtained in moderate yield. A similar temperature was needed to access the bis-propargyl ether derivative $5 i$ in $42 \%$ yield. These results may indicate a coordination of $\pi$ bonds to the cationic copper catalyst. ${ }^{[17]}$ Dissociation would be needed to allow the diazo compound to coordinate and form the metal carbene, requiring a higher reaction temperature. We then examined other O-nucleophiles than alcohols. Water was a suitable partner and gave the functionalized propargylic alcohol $5 \mathbf{j}$ in $33 \%$ yield. However, only trace amount of the desired product was observed with acetic acid, while a complex reaction mixture was obtained with phenol.

Next, we turned our attention to more complex alcoholcontaining natural products (Scheme $2 \mathrm{~B}$ ). We were pleased to see that several terpenes such as (-)-menthol and (-)-borneol were easily functionalized and gave the corresponding threecomponent products $\mathbf{5 k}$ and $\mathbf{5 l}$ in very good yields. (+)-Cedrol, possessing a tertiary alcohol, was converted to $5 \mathrm{~m}$ in $60 \%$ yield. Geraniol was a successful nucleophile partner, providing $5 \mathbf{n}$ in $57 \%$ yield, despite the presence of two potentially coordinating double bonds. Other types of natural products and biomolecules were then engaged in the three-component reaction. Notably, testosterone, protected galactose, protected thymidine and protected serine all furnished the desired propargylic ether (5o, $\mathbf{5 p}, \mathbf{5 q}$ and $\mathbf{5 r}$ ) in moderate yields showing the breadth of the scope for alcohols. 


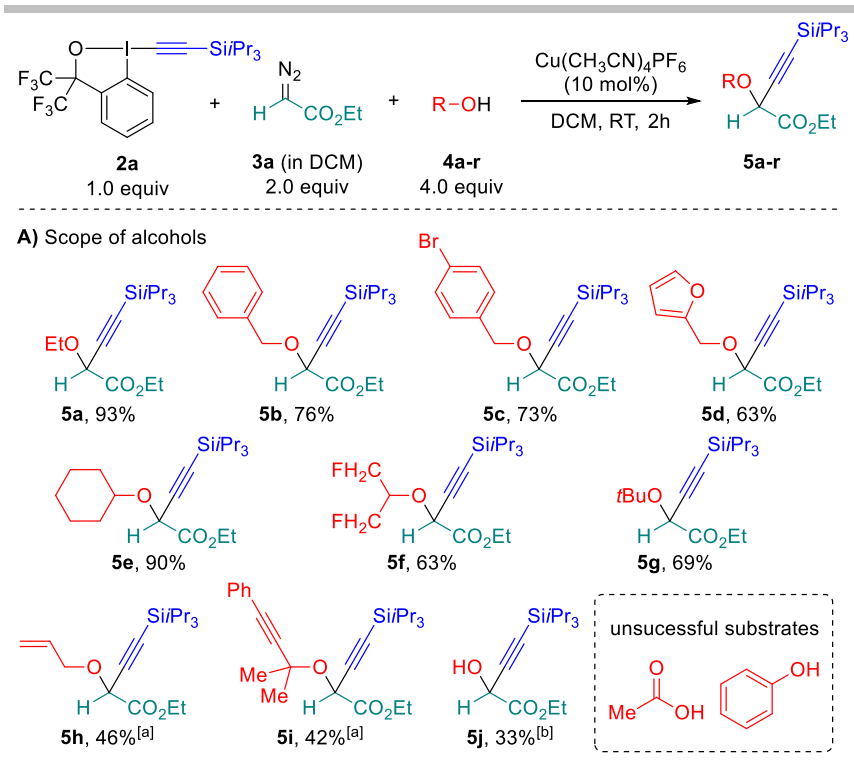

B) Scope of natural products and biomolecules
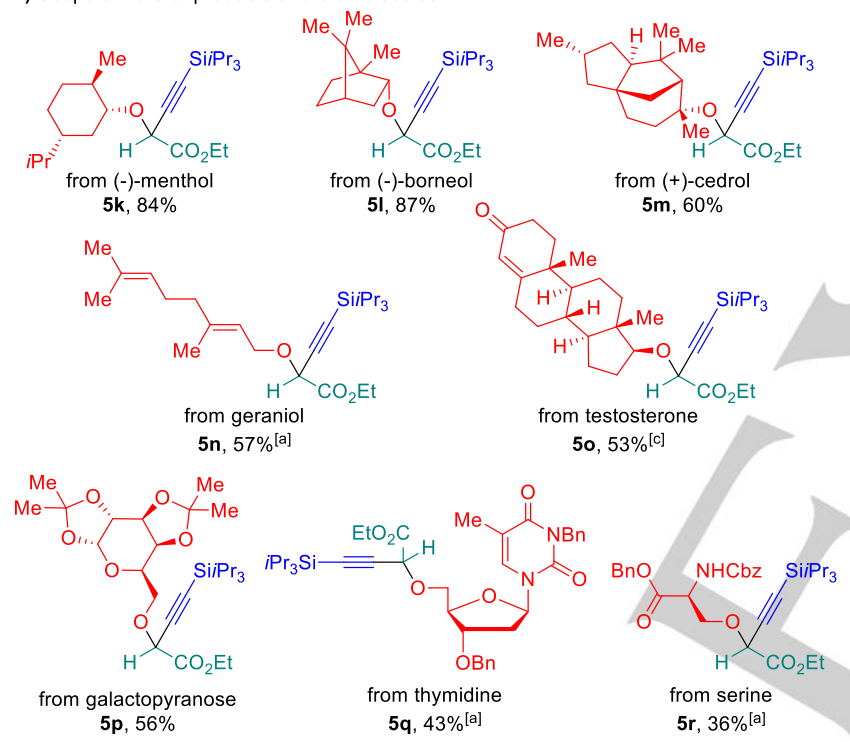

Scheme 2. Copper(I)-catalyzed three-component reaction of alcohols with ethy diazoacetate and TIPS-EBX. Unless otherwise noted, the reaction conditions are as follows: 2 a $(0.3 \mathrm{mmol})$, 3a solution in $1.0 \mathrm{~mL} \mathrm{DCM}(0.6 \mathrm{mmol}), 4 a-r(1.2$ $\mathrm{mmol}), \mathrm{DCM}(2.0 \mathrm{~mL})$. All yields refer to the isolated products. When applicable, ca. $1: 1$ d.r. was obtained. ${ }^{[a]}$ Reaction conducted at $40^{\circ} \mathrm{C}$. ${ }^{[b]} 10.0$ equiv. of water was used. ${ }^{[c]} 3.0$ equiv. of alcohol was used.

The main strength of multi-component reactions resides in the structural diversity of accessible compounds. Therefore, we decided to simultaneously vary each of the three components of the reaction to further investigate the flexibility of our methodology, (Scheme 3). We were pleased to see that diazo esters bearing various substituents, such as alkyl (5s and $\mathbf{5 t})$, aryl $(\mathbf{5 u})$, bulky aryl $(\mathbf{5 v})$ or heteroaromatic $(\mathbf{5 w})$ were tolerated. Diazo amides were not suitable reagents, furnishing only traces of the desired 3component products (not shown). Other diazo compounds bearing diverse versatile functionalities (nitrile $(\mathbf{5 x})$, phosphonate $(\mathbf{5 y})$, sulfonate $(\mathbf{5 z})$, and perfluorinated alkyls (5aa, $5 \mathbf{a b}$ and $\mathbf{5 a c})$ ) were successfully applied. The scope of EBX partners was also broad, including alkyl chains $(5 s, 5 t, 5 u$ and $5 z)$, alkenyl $(5 w)$ and aromatic substituents (5y and $5 \mathbf{a a})$ that contained several functional groups like halide (5s and $5 \mathbf{y}$ ) or a silyl ether $(\mathbf{5 z})$ and carbocycles, such as a cyclopropane $(\mathbf{5 u})$. In addition, further versatile functional groups were tolerated on the alcohols, including a bromide (5t), a ketone $(5 y)$, protected hydroxy (5u and 5aa) or a boronic ester (5ac) groups. Several carbocyclic or heterocyclic motifs important for medicinal chemistry such as cyclopropyl (5s), cyclobutyl (5z), azetidinyl (5ab), or adamantyl $(\mathbf{5 v})$ were also tolerated on the alcohol. In general, the reactions occurred smoothly, affording products in good yields. By-products and a diminished yield were obtained for the synthesis of $5 \mathbf{s}$, cyclopropyl alcohols being prone to ring-opening in presence of $\mathrm{Cu}(\mathrm{I})$ catalyst. ${ }^{[18]}$ The multiple unsaturations of substrate $5 \mathrm{w}$ could explain why only partial conversion of the EBX reagent was observed, resulting in a moderate yield.

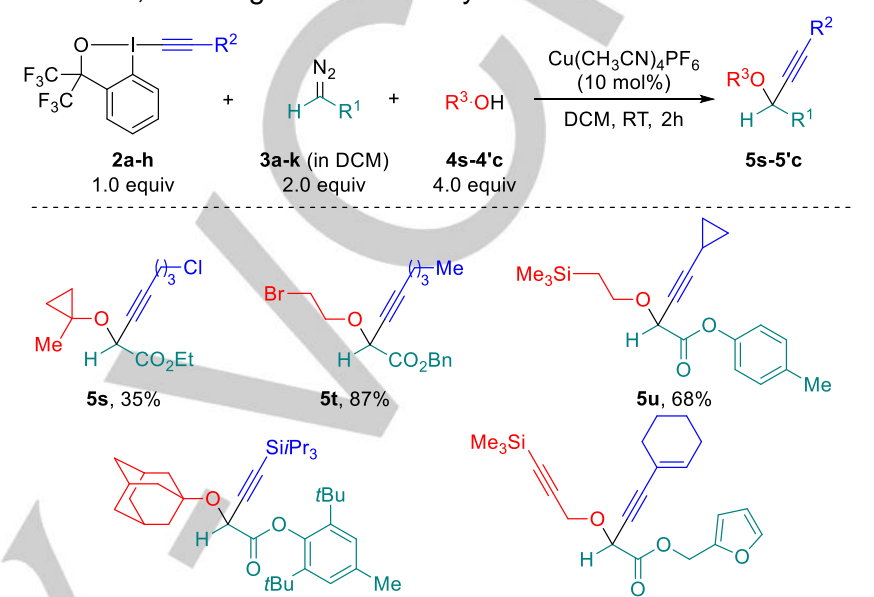

$5 v, 74 \%$

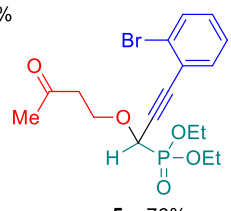

$5 w, 39 \%[a]$
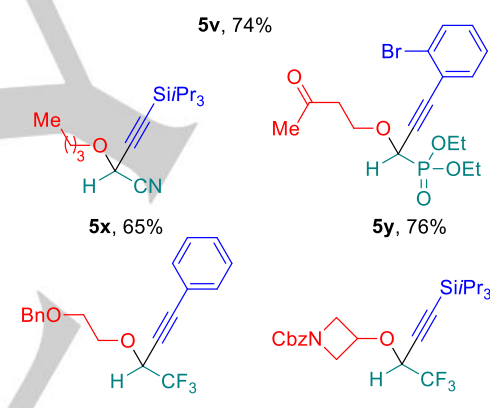

5aa, $73 \%$

$5 \mathrm{ab}, 61 \%^{[\mathrm{a}]}$
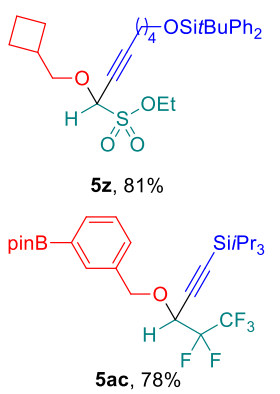

Scheme 3. Substrate scope with simultaneous variation of the three components. Unless otherwise noted, the reaction conditions are as follows: $2 \mathrm{a}$ h $(0.3 \mathrm{mmol})$, 3a-k solution in $1.0 \mathrm{~mL} \mathrm{DCM}(0.6 \mathrm{mmol}), 4 \mathrm{~s}-4 \mathrm{ac}(1.2 \mathrm{mmol})$, DCM $(2.0 \mathrm{~mL})$. All yields refer to the isolated products. ${ }^{\left[{ }^{[a]}\right.}$ Reaction conducted at $40^{\circ} \mathrm{C}$.

Next, we used disubstituted diazo compounds (Scheme 4A). Diazo compounds bearing a methyl or a phenyl substituent furnished $5 \mathrm{ad}$ and $5 \mathrm{ae}$ in $43 \%$ and $57 \%$ yield respectively, without the need to reoptimize the reaction conditions. A cyclic diazo compound provided $\mathbf{5 a f}$ in moderate yield. Finally, we investigated a substrate having a pendant hydroxy group for intramolecular nucleophile attack. The desired tetrahydropyran 5 ag was formed, albeit in low yield.

The high efficiency of the three-component reaction with trifluoromethyl-substituted diazo compounds is especially interesting, as organofluorine compounds are of significant importance in the pharmaceutical, agrochemical and materials industry ${ }^{[19]}$ For example, Efavirenz (10), which contains a $\mathrm{CF}_{3-}$ propargyl motif, is one of the most frequently prescribed antiretroviral drug used in HIV treatment. We decided to apply our methodology to access the Lonza intermediate $5 \mathrm{ah}$, a direct precursor to Efavirenz (10) (Scheme 4B). ${ }^{[20]}$ We envisaged the use of tert-butyl carbamate as nucleophile, anticipating that $\mathrm{O}$ attack followed by loss of the tert-butyl group could generate the desired $\mathrm{NH}_{2}$-carbamate. In this case, the number of equivalents of reagents, as well as the catalyst loading, could be reduced as 
there was no competitive $\mathrm{O}-\mathrm{H}$ insertion pathway. The desired molecule 5ah was obtained in good yield without the formation of products from $\mathrm{N}-\mathrm{H}$ insertion. ${ }^{[21]}$ Our methodology also gave access to structurally diverse analogues. The polytrifluoromethylated compound 5ai, bearing a thiophene on the alkyne, was synthesized in good yield, as well as ether 5aj bearing a nitrile group. ${ }^{[22]}$ Finally, the carbamate derivative 5ak containing a protected amine and an aryl bromide was obtained in $31 \%$ yield.

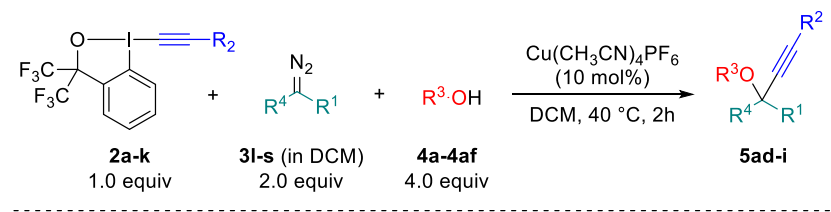

A) Scope with disubstituted diazo compounds
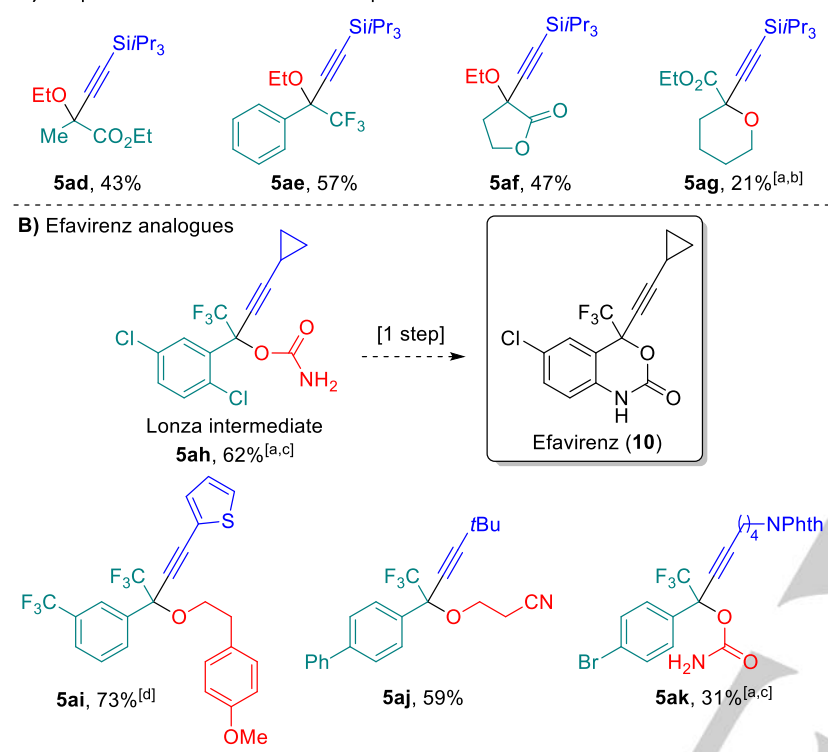

Scheme 4. Substrate scope with disubstituted diazo compounds and Efavirenz analogues. Unless otherwise noted, the reaction conditions are as follows: $2 a-$ $\mathbf{k}(0.3 \mathrm{mmol})$, 3I-s solution in $1.0 \mathrm{~mL}$ DCM $(0.6 \mathrm{mmol})$, 4a-4af $(1.2 \mathrm{mmol}), \mathrm{DCM}$ $(2.0 \mathrm{~mL})$. All yields refer to the isolated products. ${ }^{\text {[a] The reaction was conducted }}$ at RT. ${ }^{[b]} \mathrm{No}$ alcohol was used. ${ }^{\left[{ }^{[c} \mathrm{BocNH}_{2}\right.}$ 4ad (1.3 equiv), 3p-s (1.3 equiv) and $\mathrm{Cu}\left(\mathrm{CH}_{3} \mathrm{CN}\right)_{4} \mathrm{PF}_{6}(5 \mathrm{~mol} \%)$ were used. ${ }^{[d]}$ Yield determined by ${ }^{19} \mathrm{~F}$ NMR spectroscopy.

To gain insights into the mechanism, several control experiments were carried out (Scheme 5A). First, when ethyl diazoacetate (3a) was reacted with ethanol (4a) in presence of $10 \mathrm{~mol} \%$ of $\mathrm{Cu}\left(\mathrm{CH}_{3} \mathrm{CN}\right)_{4} \mathrm{PF}_{6}$, we observed the formation of $6 \mathbf{a}$ by ${ }^{1} \mathrm{H} N M R$ spectroscopy analysis of the reaction mixture (Eq. (1)). However, no three-component product $\mathbf{5 a}$ was observed after the subsequent addition of TIPS-EBX (2a) to this solution. This indicates that $\mathbf{6 a}$ is not an intermediate in the catalytic cycle. When mixing reagent $\mathbf{2 a}$ and diazo $\mathbf{3 a}$ in the presence of the copper catalyst, rapid evolution of nitrogen occurred and we mainly observed the formation of diethyl fumarate/maleate (11) (Eq. (2)). ${ }^{[3 d]}$ Other products resulting from a minor decomposition (ca. $10 \%$ ) of the hypervalent iodine reagent $2 \mathrm{a}$ could not be identified. $\mathrm{A}{ }^{1} \mathrm{H}$ NMR titration experiment was then carried out using $\mathrm{Cu}\left(\mathrm{CH}_{3} \mathrm{CN}\right)_{4} \mathrm{PF}_{6}$ in combination with EBX 2a. Progressive shifts of the aromatic ${ }^{1} \mathrm{H}$ were observed upon addition of the copper salt, with a significant diminution after one equivalent (See Figure $\mathrm{S} 3$ in Supporting Information). $A{ }^{13} \mathrm{C}$ NMR spectrum of an equimolar mixture of $\mathrm{Cu}\left(\mathrm{CH}_{3} \mathrm{CN}\right)_{4} \mathrm{PF}_{6}$ and $2 \mathrm{a}$ in $\mathrm{CD}_{2} \mathrm{Cl}_{2}$ showed major changes in the ${ }^{13} \mathrm{C}$-alkyne signals, while other peaks remained nearly constant (See Figure $\mathrm{S} 5$ in Supporting Information). When ethanol (4 equiv.) and $\mathrm{Cu}\left(\mathrm{CH}_{3} \mathrm{CN}\right)_{4} \mathrm{PF}_{6}(1$ equiv.) were mixed together in $\mathrm{CD}_{2} \mathrm{Cl}_{2}$, no ligand exchange was observed (See Figure S6 in Supporting Information). These NMR studies indicated an interaction between the $\mathrm{Cu}$ catalyst and the EBX reagent $2 a$, probably through the alkyne, but no complete reaction, making oxidative addition of the reagent onto copper to form a $\mathrm{Cu}$ (III) intermediate a less probable pathway. ${ }^{\left[{ }^{[3]}\right]}$ We then attempted the trapping of a potential Cu-carbene intermediate through a cyclopropanation reaction using one equivalent of ethyl diazoacetate (3a) and 4 equivalents of ethanol (4a) and styrene (Eq. (3)). Cylcopropane 12 was obtained in $21 \%$ NMR yield, and the three-component product 5 a was formed in $62 \%$ yield. Aside from supporting the existence of a metal carbene, this competitive experiment, also shows that attack of ethanol to form a putative oxonium ylide is faster than cyclopropanation. Finally, we reexamined the reaction of $\mathbf{2 a}, \mathbf{3} \mathbf{a}$ and benzyl alcohol $(\mathbf{4 b})$ in presence of chiral ligand 9 under the optimized reaction conditions. The product $\mathbf{5 a}$ was obtained in poor yield as a racemic compound (Eq. (4)). This may indicate that ligand decomplexation is required for the reaction to proceed, making the development of an enantioselective method highly challenging.

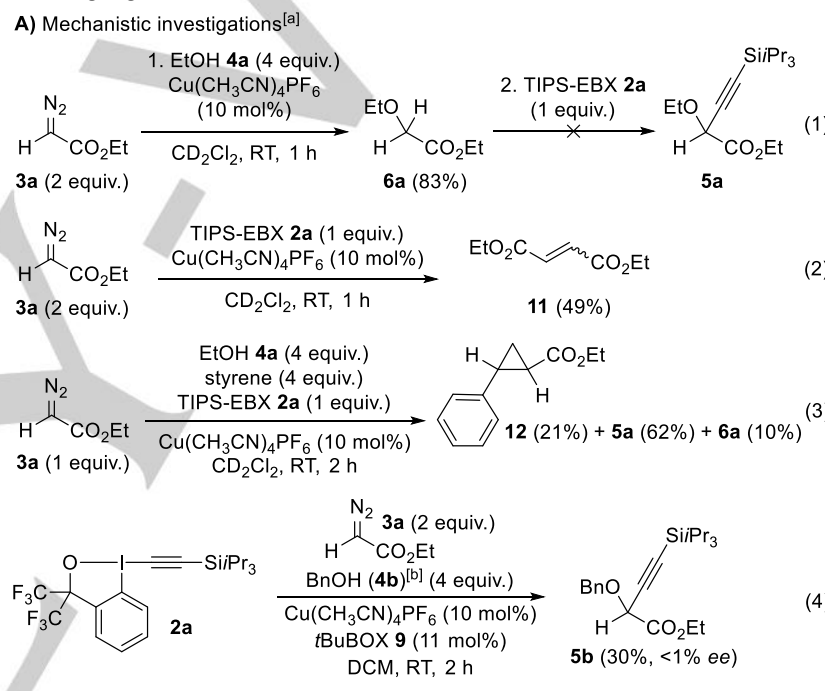

Proposed catalytic cycle

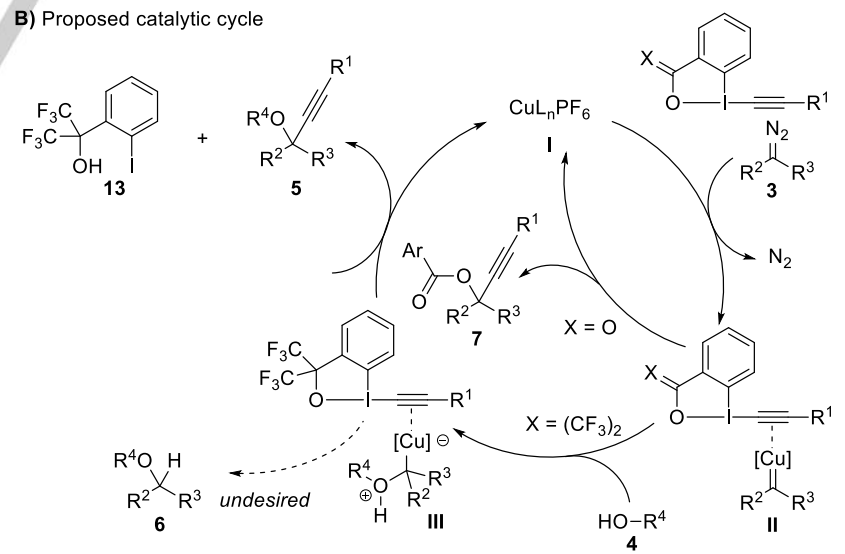

Scheme 5. Mechanistic studies and proposed catalytic cycle. ${ }^{[a]}$ Yields determined by ${ }^{1} \mathrm{H}$ NMR analysis of the crude reaction mixture.

Based on the results of our control experiments and the relevant literature on metal-ylide based multicomponent reactions, ${ }^{[3]}$ our proposed catalytic cycle is presented in Scheme 5B. Starting with $\mathrm{Cu}(\mathrm{I})$ catalyst $\mathrm{I}$, reaction with diazo compound $\mathbf{3}$ and the hypervalent iodine reagents generates electrophilic copper-carbene II with alkyne-bound EBX. At this stage, it is difficult to establish if coordination of the hypervalent iodine 
reagents is occurring before or after carbene formation. From II, two pathways are possible: With carboxylate reagent $\mathbf{1}$, intramolecular oxygen attack directly followed by alkynylation leads to two-component product 7; With bis-trifluoromethyl reagents 2 , the oxygen atom of the benziodoxole is not nucleophilic enough, and attack of the external alcohol $\mathbf{4}$ forms oxonium-ylide intermediate III. Intramolecular alkynylation by EBX reagent 2 with simultaneous deprotonation then generates the 3 component product 5 and iodide 13, releasing the copper catalyst. Undesired 1,2-H shift on the transient ylide III produces the $\mathrm{O}-\mathrm{H}$ insertion product 6 . Further studies will be needed to establish the mechanism of the alkynylation step.

In conclusion, we have reported a general copper-catalyzed three-component reactions of alcohols, diazo compounds and alkynyl benziodoxole reagents. The reaction can be done under mild conditions and each of the three partners can be extensively varied, leading to maximal structural diversity. Preliminary mechanism investigations support a mechanism involving subsequent formation of copper carbene and ylide intermediates, followed by electrophilic alkynylation. Future work in our laboratory will focus on a more in-depth understanding of the reaction mechanism, the development of an enantioselective variation and the use of other classes of nucleophilic partners in the three-component transformation.

\section{Acknowledgements}

We thank the Swiss National Science Foundation (Grant No. 200021_165788) and EPFL for financial support. We thank Dr R. Scopelliti and Dr F. F. Tirani from ISIC at EPFL for X-ray analysis.

Keywords: multi-component reactions (MCR); copper catalysis; molecular complexity; hypervalent iodine reagents; carbenes.

[1] Multicomponent Reactions, Ed. J. Zhu, H. Bienaymé, Wiley-VCH Verlag, 2005

[2] a) A. Domling, I. Ugi, Angew. Chem., Int. Ed. 2000, 39, 3168; b) B. B. Toure, D. G. Hall, Chem. Rev. 2009, 109, 4439; c) E. Ruijter, R Scheffelaar, R. V. A. Orru, Angew. Chem., Int. Ed. 2011, 50, 6234; d) A. Domling, W. Wang, K. Wang, Chem. Rev. 2012, 112, 3083; e) Q. Wang D. X. Wang, M. X. Wang, J. P. Zhu, Acc. Chem. Res. 2018, 51, 1290.

[3] a) X. Guo, W. Hu, Acc. Chem. Res. 2013, 46, 2427; b) D. F. Chen, Z. Y. Han, X. L. Zhou, L. Z. Gong, Acc. Chem. Res. 2014, 47, 2365; c) D. Zhang, W. H. Hu, Chem. Rec. 2017, 17, 739; For a general review on the use of diazo compounds, see: A. Ford, H. Miel, A. Ring, C. N. Slattery, A. R. Maguire, M. A. McKervey, Chem. Rev. 2015, 115, 9981.

[4] Selected examples: a) H. Huang, X. Guo, W. Hu, Angew. Chem., Int. Ed. 2007, 46, 1337; b) X. Y. Guan, L. P. Yang, W. H. Hu, Angew. Chem., Int Ed. 2010, 49, 2190; c) H. Qiu, M. Li, L. Q. Jiang, F. P. Lv, L. Zan, C. W Zhai, M. P. Doyle, W. H. Hu, Nat. Chem. 2012, 4, 733; d) S. Jia, D. Xing D. Zhang, W. Hu, Angew. Chem., Int. Ed. 2014, 53, 13098; e) X. C. Ma J. Jiang, S. Y. Lv, W. F. Yao, Y. Yang, S. Y. Liu, F. Xia, W. H. Hu, Angew. Chem., Int. Ed. 2014, 53, 13136; f) X. Lv, Z. Kang, D. Xing, W. Hu, Org. Lett. 2018, 20, 4843; g) J. Che, A. Gopi Krishna Reddy, L. Niu, D. Xing W. Hu, Org. Lett. 2019, 21, 4571; h) S. Yu, R. Hua, X. Fu, G. Liu, D. Zhang, S. Jia, H. Qiu, W. Hu, Org. Lett. 2019, 21, 5737; i) W. Hu, X. Xu J. Zhou, W.-J. Liu, H. Huang, J. Hu, L. Yang, L.-Z. Gong, J. Am. Chem. Soc. 2008, 130, 7782; j) D. F. Chen, F. Zhao, Y. Hu, L. Z. Gong, Angew. Chem., Int. Ed. 2014, 53, 10763; k) Y. Xia, S. Feng, Z. Liu, Y. Zhang, J. Wang, Angew. Chem., Int. Ed. 2015, 54, 7891; I) C. Wang, F. Ye, C. Wu, Y. Zhang, J. Wang, J. Org. Chem 2015, 80, 8748; m) C. G. Wu, Z. X. Liu Z. K. Zhang, F. Ye, G. S. Deng, Y. Zhang, J. B. Wang, Adv. Synth. Catal. 2016, 358, 2480; n) S. K. Alamsetti, M. Spanka, C. Schneider, Angew. Chem., Int. Ed. 2016, 55, 2392; o) S. Goudedranche, C. Besnard, L. Egger, J. Lacour, Angew. Chem., Int. Ed. 2016, 55, 13775; p) W. Yuan, K. J. Szabó, ACS Catalysis 2016, 6, 6687; q) M. Lübcke, W. Yuan, K. J.
Szabó, Org. Lett. 2017, 19, 4548; r) M. Lübcke, D. Bezhan, K. J. Szabó, Chemical Science 2019, 10, 5990; s) X.-S. Liang, R.-D. Li, X.-C. Wang Angew. Chem., Int. Ed. 2019, 58, 13885; t) J. Yu, L. Chen, J. Sun, Org. Lett. 2019, 21, 1664.

[5] A. Yoshimura, V. V. Zhdankin, Chem. Rev 2016, 116, 3328-3435.

[6] a) J. Kaschel, D. B. Werz, Angew. Chem., Int. Ed. 2015, 54, 8876; b) Y. Li, D. P. Hari, M. V. Vita, J. Waser, Angew. Chem., Int. Ed. 2016, 55, 4436; c) D. P. Hari, P. Caramenti, J. Waser, Acc. Chem. Res. 2018, 51, 3212 .

[7] a) J. Tao, R. Tran, G. K. Murphy, J. Am. Chem. Soc. 2013, 135, 16312 b) G. K. Murphy, F. Z. Abbas, A. V. Poulton, Adv. Synth. Catal. 2014, 356, 2919; c) G. S. Sinclair, R. Tran, J. Tao, W. S. Hopkins, G. K. Murphy, Eur. J. Org. Chem. 2016, 4603; d) Z. Zhao, K. G. Kulkarni, G. K. Murphy, Adv. Synth. Catal. 2017, 359, 2222.

[8] N. Döben, H. Yan, M. Kischkewitz, J. Mao, A. Studer, Org. Lett. 2018, 20, 7933.

[9] D. Zhu, Y. Yao, R. Zhao, Y. Liu, L. Shi, Chem. Eur. J. 2018, 24, 4805

[10] a) D. P. Hari, J. Waser, J. Am. Chem. Soc. 2016, 138, 2190; b) D. P. Hari, J. Waser, J. Am. Chem. Soc. 2017, 139, 8420; c) D. P. Hari, L. Schouwey, V. Barber, R. Scopelliti, F. Fadaei-Tirani, J. Waser, Chem. Eur. J. 2019, 25, 9522.

[11] a) W. Yuan, L. Eriksson, K. J. Szabó, Angew. Chem., Int. Ed. 2016, 55, 8410 ; b) B. K. Mai, K. J. Szabó, F. Himo, ACS Catal. 2018, 8, 4483

[12] For selected examples of propargylic ether synthesis, see: a) E. B. Bauer, Synthesis 2012, 44, 1131; b) Y. X. Zhu, L. Sun, P. Lu, Y. G. Wang, ACS Catal. 2014, 4, 1911; c) M. Baxter, Y. Bolshan, Chem. Eur. J. 2015, 21, 13535; e) K. Nakajima, M. Shibata, Y. Nishibayashi, J. Am. Chem. Soc 2015, 137, 2472.

[13] a) V. V. Zhdankin, C. J. Kuehl, A. P. Krasutsky, J. T. Bolz, A. J. Simonsen J. Org. Chem. 1996, 61, 6547; b) Y. Li, J. P. Brand, J. Waser, Angew. Chem., Int. Ed. 2013, 52, 6743; c) Y. Li, J. Waser, Angew. Chem., Int Ed. 2015, 54, 5438; d) J. Wu, N. Yoshikai, Angew. Chem., Int. Ed. 2015, $54,11107$.

[14] No product 5a was obtained when using Cul, CuCN $\mathrm{Cu}(\mathrm{II})$ trifluoroacetylycetonate or $\mathrm{Cu}(\mathrm{OAc})_{2}$ as catalyst.

[15] On scope scale, better results were obtained when diazo compound 3 was added slowly as a solution in $\mathrm{CH}_{2} \mathrm{Cl}_{2}(0.6 \mathrm{M}$, addition rate $1 \mathrm{~mL} / \mathrm{h})$.

[16] H. C. Beyerman, G. J. Heiszwolf, Recl. Trav. Chim. Pays-Bas 1965, 84, 203.

[17] R. G. Salomon, J. K. Kochi, J. Am. Chem. Soc. 1973, 95, 3300.

[18] H. Zhang, G. Wu, H. Yi, T. Sun, B. Wang, Y. Zhang, G. Dong, J. Wang, Angew. Chem. Int. Ed. 2017, 56, 3945.

[19] Organofluorine Compounds in Biology and Medicine, V. P. Reddy, Elsevier, 2015.

[20] D. Dai, X. Long, A. Kulesza, J. Reichwagen, B. Luo and Y. Guo (Lonza Ltd), PCT Int. Appl. WO2012097510, 2012.

[21] E. C. Lee, G. C. Fu, J. Am. Chem. Soc. 2007, 129, 12066

[22] The structure of 5 aj was confirmed by X-ray analysis. The structure is available at the Cambridge Crystallographic Data Center (ccdc number: 1985456).

[23] The formation of $\mathrm{Cu}$ (III) intermediates by reaction oxidation with hypervalent iodine reagents has been proposed, see for example: a) $D$. Holt, M. J. Gaunt, Angew. Chem. Int. Ed. 2015, 54, 7857; b) M. G. Suero, E. D.Bayle, B. S. L. Collins, M. J. Gaunt, J. Am. Chem. Soc. 2013, 135, 5332; c) A. J. Hickman, M. S. Sanford, Nature 2012, 484, 177. 


\section{COMMUNICATION}
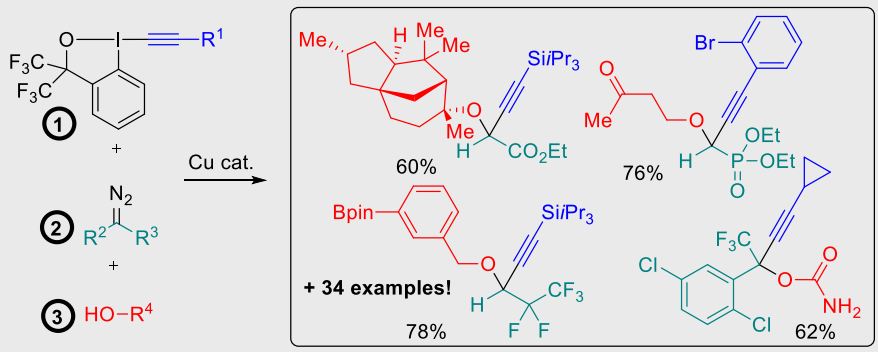

Guillaume Pisella, Alec Gagnebin and Jerome Waser*

\section{Page No. - Page No.}

Three-Component Reaction for the Synthesis of Highly Functionalized Propargyl Ethers

Power 3: A copper-catalyzed three-component reaction of hypervalent iodine reagents, diazo compounds and alcohols has been developed. The transformation gives access to functionalized propargylic ethers with high structural diversity, as variation of the three partners with numerous functional groups was tolerated. The reaction is speculated to proceed via a copper oxonium ylide intermediate. 
Supporting Information

(110 pages)

Authors contributions: G. P. performed and planned the experiments and prepared the manuscript and the experimentals part, A. G. performed the experiments as a laboratory technician in formation under the supervision of G. P., J. W. supervised the project, prepared the manuscript and corrected the experimental part.

\section{Table of Contents}

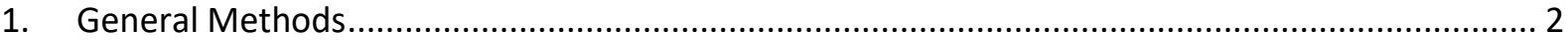

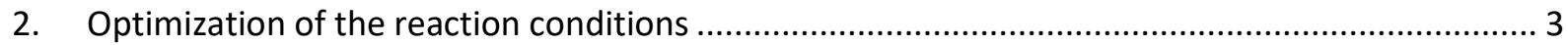

3. 3-component reaction of alcohols, diazo compounds and EBX reagents .................................... 7

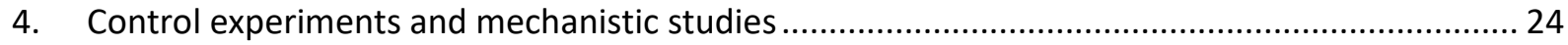

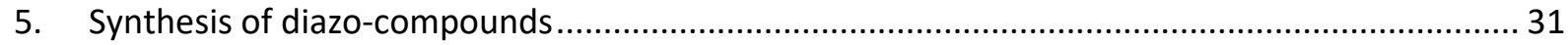

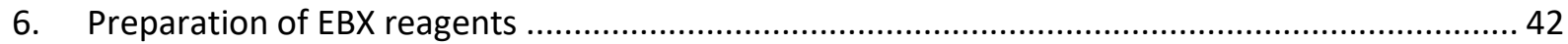

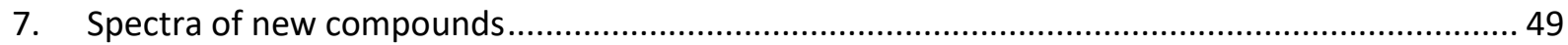

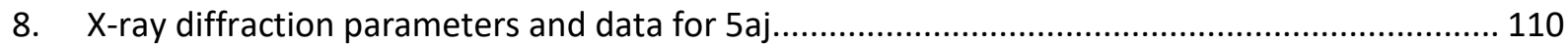




\section{General Methods}

All reactions were carried out in oven dried glassware under an atmosphere of nitrogen, unless stated otherwise. For quantitative flash chromatography technical grade solvents were used. For flash chromatography for analysis, HPLC grade solvents from Sigma-Aldrich were used. THF, $\mathrm{Et}_{2} \mathrm{O}, \mathrm{CH}_{3} \mathrm{CN}$, toluene, hexane and $\mathrm{CH}_{2} \mathrm{Cl}_{2}$ were dried by passage over activated alumina under nitrogen atmosphere $\left(\mathrm{H}_{2} \mathrm{O}\right.$ content $<10 \mathrm{ppm}$, Karl-Fischer titration). The solvents were degassed by Freeze-Pump-Thaw method when mentioned. All chemicals were purchased from Acros, Aldrich, Fluka, Fluorochem, TCl, VWR or Merck and used as such unless stated otherwise. Chromatographic purification was performed as flash chromatography using Macherey-Nagel silica 40-63, $60 \AA$, using the solvents indicated as eluent with 0.1-0.5 bar pressure. TLC was performed on Merck silica gel 60 F254 TLC aluminium plates and visualized with UV light, permanganate stain or 4-anisaldehyde stain. Melting points were measured on a Büchi B-540 melting point apparatus using open glass capillaries, the data is uncorrected. ${ }^{1} \mathrm{H}$ NMR spectra were recorded on a Brucker DPX-400 $400 \mathrm{MHz}$ spectrometer in $\mathrm{CDCl}_{3}$, DMSO- $d_{6}, \mathrm{CD}_{2} \mathrm{Cl}_{2}$ or $\mathrm{CD}_{3} \mathrm{OD}$, all signals are reported in ppm with the internal chloroform signal at 7.26 $\mathrm{ppm}$, the internal DMSO signal at $2.50 \mathrm{ppm}$, the internal dichloromethane signal at $5.32 \mathrm{ppm}$ or the internal methanol signal at $3.30 \mathrm{ppm}$ as standard. The data is being reported as ( $\mathrm{s}=$ singlet, $\mathrm{d}=$ doublet, $\mathrm{t}=$ triplet, $\mathrm{q}=$ quadruplet, $\mathrm{qi}=$ quintet, $\mathrm{m}=$ multiplet or unresolved, $\mathrm{br}=$ broad signal, $\mathrm{app}=$ apparent, coupling constant(s) in $\mathrm{Hz}$, integration, interpretation). ${ }^{13} \mathrm{C}$ NMR spectra were recorded with ${ }^{1} \mathrm{H}$ decoupling on a Brucker DPX-400 $100 \mathrm{MHz}$ spectrometer in $\mathrm{CDCl}_{3}$, DMSO- $d_{6}, \mathrm{CD}_{2} \mathrm{Cl}_{2}$, or $\mathrm{CD}_{3} \mathrm{OD}$, all signals are reported in ppm with the internal chloroform signal at $77.0 \mathrm{ppm}$, the internal DMSO signal at $39.5 \mathrm{ppm}$, the internal dichloromethane signal at $54.0 \mathrm{ppm}$ or the internal methanol signal at 49.0 ppm as standard. Infrared spectra were recorded on a JASCO FT-IR B4100 spectrophotometer with an ATR PRO410-S and a ZnSe prisma and are reported as $\mathrm{cm}^{-1}$ ( $\mathrm{w}=$ weak, $\mathrm{m}=$ medium, $\mathrm{s}=$ strong, $\mathrm{br}=$ broad). High resolution mass spectrometric measurements were performed by the mass spectrometry service of ISIC at the EPFL on a MICROMASS (ESI) Q-TOF Ultima API. HPLC measurements were done on a Agilent 1260 Infinity autosampler using a CHIRALPAK IA column from DAICEL Chemical. 


\section{Optimization of the reaction conditions}

a) Screening of the electrophilic alkynyl source, catalyst loading and ligands

In a $\mathrm{N}_{2}$-filled glovebox, a catalytic solution was prepared by mixing $\mathrm{Cu}\left(\mathrm{CH}_{3} \mathrm{CN}\right)_{4} \mathrm{BF}_{4}(\mathrm{x} \mathrm{mol} \%)$ and ligand (8 - 9) (y mol\%) (when applicable) in $\mathrm{CD}_{2} \mathrm{Cl}_{2}(2.00 \mathrm{~mL}$ ) at room temperature for $1 \mathrm{~h}$.

The catalytic solution was prepared in $\mathrm{EtOH}$ when used as solvent.

$0.40 \mathrm{~mL}$ of the catalytic solution was then added to a stirring solution of hypervalent iodine reagent ( $\mathbf{1}$, 2a or 14) (0.08 mmol, 1.00 equiv.), EtOH (4a) (w equiv.) and ethyl diazoacetate (3a) (v equiv.) in $\mathrm{CD}_{2} \mathrm{Cl}_{2}$ $(0.40 \mathrm{~mL})$. The resulting reaction mixture was stirred at room temperature for $1 \mathrm{~h}$. After this time, a ${ }^{1} \mathrm{H}$ NMR spectrum of the reaction mixture was recorded. Yields were obtained by comparing the integration of the signals at $8.43-8.36 \mathrm{ppm}$ for $2 \mathrm{a}, 8.17 \mathrm{ppm}$ for the corresponding alcohol, $4.72 \mathrm{ppm}$ for $5 a$ and 4.03 ppm for $6 a$, and are not calibrated.

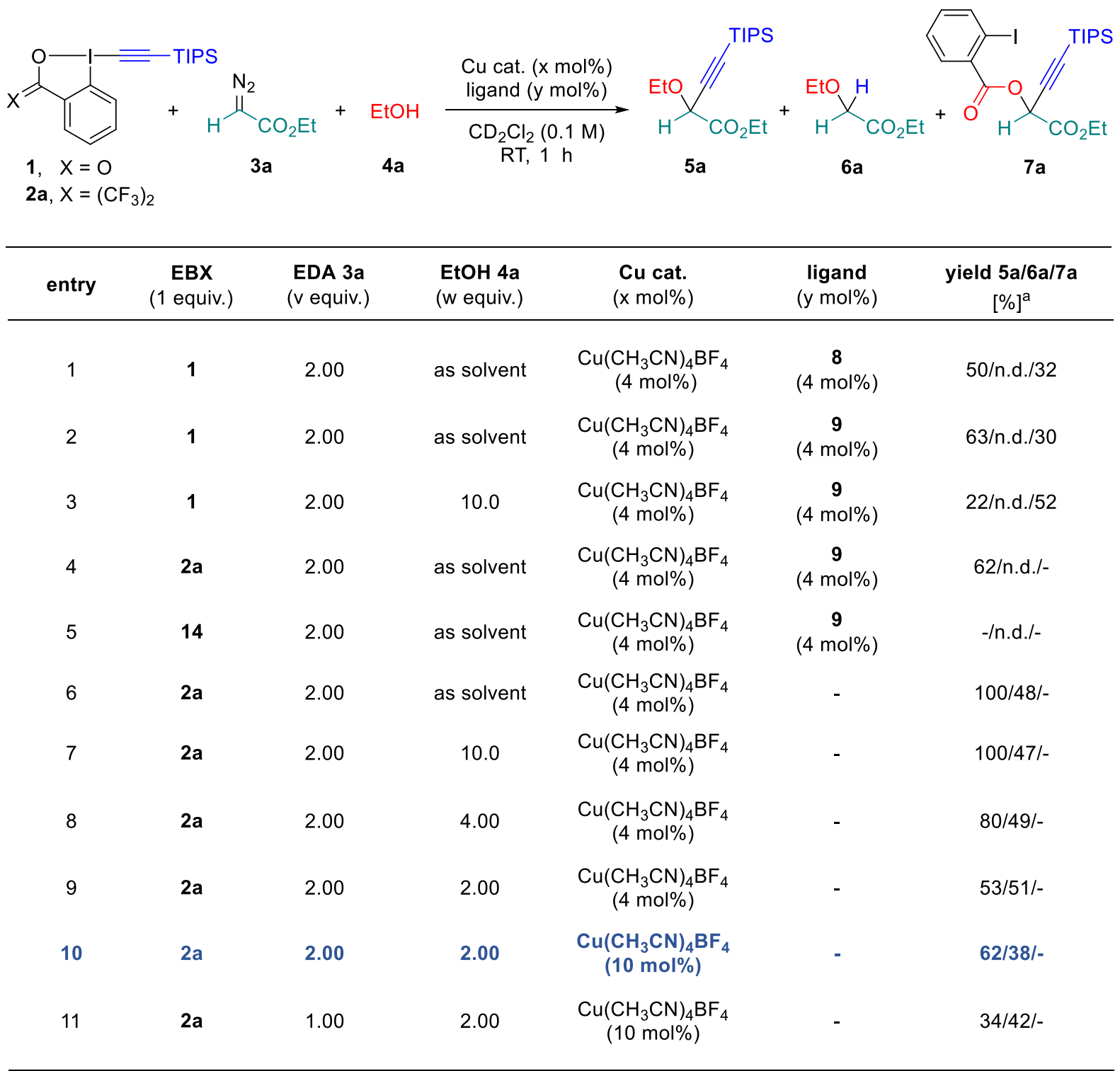

[a] Determined by ${ }^{1} \mathrm{H}$ NMR analysis<smiles>Clc1cccc(Cl)c1/C=N/CC/N=C/c1c(Cl)cccc1Cl</smiles><smiles>CC(C)[C@H]1COC(C(C)(C)C2=N[C@H](C(C)C)CO2)=N1</smiles><smiles>C[AsH2]=S</smiles> 
It was found that reagent $\mathbf{2} \mathbf{a}$ was converted only to the desired three-component product $\mathbf{5 a}$. The absence of a ligand was beneficial for the formation of $\mathbf{5 a}$. Increasing the $\mathrm{Cu}$ catalyst loading to 10 mol\% improved the formation of $\mathbf{5 a}$.

b) Screening of additives

In a $\mathrm{N}_{2}$ filled glovebox, an oven-dried $10 \mathrm{~mL}$ microwave vial was charged with $\mathrm{Cu}\left(\mathrm{CH}_{3} \mathrm{CN}_{4}\right)_{4} \mathrm{BF}_{4}(2.52 \mathrm{mg}$, $8.00 \mu \mathrm{mol}, 0.10$ equiv.), TIPS-EBX $2 \mathrm{a}(44.0 \mathrm{mg}, 80.0 \mu \mathrm{mol}, 1.00$ equiv.) and the additive (x equiv.), if solid. The vial was capped, removed from the glovebox and $\mathrm{CD}_{2} \mathrm{Cl}_{2}(0.80 \mathrm{~mL})$ was added, followed by EtOH (4a) ( $9.34 \mu \mathrm{L}, 160 \mu \mathrm{mol}, 2.00$ equiv.), the additive (x equiv.), if liquid, and ethyl diazoacetate (3a) $(19.0 \mu \mathrm{L}, 160 \mu \mathrm{mol}, 2.00$ equiv., 87\%wt in DCM). The resulting reaction mixture was stirred at room temperature for $1 \mathrm{~h}$. After this time, ${ }^{1} \mathrm{H}$ NMR spectrum of the reaction mixture was recorded.

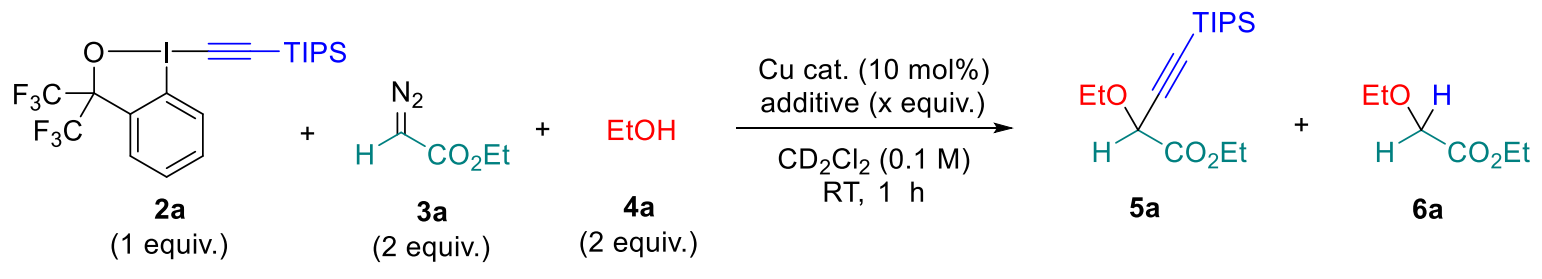

\begin{tabular}{|c|c|c|c|}
\hline entry & $\begin{array}{l}\text { Cu cat. } \\
(10 \mathrm{~mol} \%)\end{array}$ & $\begin{array}{l}\text { additive } \\
\text { (x equiv.) }\end{array}$ & $\begin{array}{c}\text { yield } 5 a / 6 a \\
{[\%]^{a}}\end{array}$ \\
\hline 1 & $\mathrm{Cu}\left(\mathrm{CH}_{3} \mathrm{CN}\right)_{4} \mathrm{BF}_{4}$ & $\begin{array}{c}\text { proton sponge (15) } \\
(2.00)\end{array}$ & no reaction \\
\hline 2 & $\mathrm{Cu}\left(\mathrm{CH}_{3} \mathrm{CN}\right)_{4} \mathrm{BF}_{4}$ & $\begin{array}{l}\mathrm{LiOH} \\
(2.00)\end{array}$ & no reaction \\
\hline 3 & $\mathrm{Cu}\left(\mathrm{CH}_{3} \mathrm{CN}\right)_{4} \mathrm{BF}_{4}$ & $\begin{array}{c}\mathrm{Cs}_{2} \mathrm{CO}_{3} \\
(2.00)\end{array}$ & no reaction \\
\hline 4 & $\mathrm{Cu}\left(\mathrm{CH}_{3} \mathrm{CN}\right)_{4} \mathrm{BF}_{4}$ & $\begin{array}{l}\mathrm{NaOAc} \\
(2.00)\end{array}$ & no reaction \\
\hline 5 & $\mathrm{Cu}\left(\mathrm{CH}_{3} \mathrm{CN}\right)_{4} \mathrm{BF}_{4}$ & $\begin{array}{c}\mathrm{NaHCO}_{3} \\
(2.00)\end{array}$ & $50 / 36$ \\
\hline 6 & $\mathrm{Cu}\left(\mathrm{CH}_{3} \mathrm{CN}\right)_{4} \mathrm{BF}_{4}$ & $\begin{array}{l}\text { HFIP } \\
(40.0)\end{array}$ & $0 / 100$ \\
\hline 7 & $\mathrm{Cu}\left(\mathrm{CH}_{3} \mathrm{CN}\right)_{4} \mathrm{BF}_{4}$ & $\begin{array}{l}\text { CPA (16) } \\
(5 \mathrm{~mol} \%)\end{array}$ & $13 / 85$ \\
\hline
\end{tabular}

[a] Determined by ${ }^{1} \mathrm{H}$ NMR analysis<smiles>CN(C)c1cccc2cccc(N(C)C)c12</smiles>

(15)<smiles>O=P(O)(O)Oc1ccc2ccccc2c1-c1c(O)ccc2ccccc12</smiles>

None of the additives screened above were found to improve the formation of the three-component product $\mathbf{5 a}$.

c) Screening of the copper catalysts

In a $\mathrm{N}_{2}$ filled glovebox, an oven-dried $10 \mathrm{~mL}$ microwave vial was charged with $\mathrm{Cu}$ cat. $(8.00 \mu \mathrm{mol}, 0.10$ equiv.) and TIPS-EBX 2a (44.0 mg, $80.0 \mu \mathrm{mol}, 1.00$ equiv.). The vial was capped, removed from the glovebox and $\mathrm{CD}_{2} \mathrm{Cl}_{2}(0.80 \mathrm{~mL})$ was added, followed by $\mathrm{EtOH}(4 \mathrm{a})(9.30 \mu \mathrm{L}, 160 \mu \mathrm{mol}, 2.00$ equiv.) and ethyl diazoacetate (3a) $(19.0 \mu \mathrm{L}, 160 \mu \mathrm{mol}, 2.00$ equiv., $87 \% \mathrm{wt}$ in DCM). The resulting reaction mixture 
was stirred at room temperature for $1 \mathrm{~h}$. After this time, ${ }^{1} \mathrm{H}$ NMR spectrum of the reaction mixture was recorded.

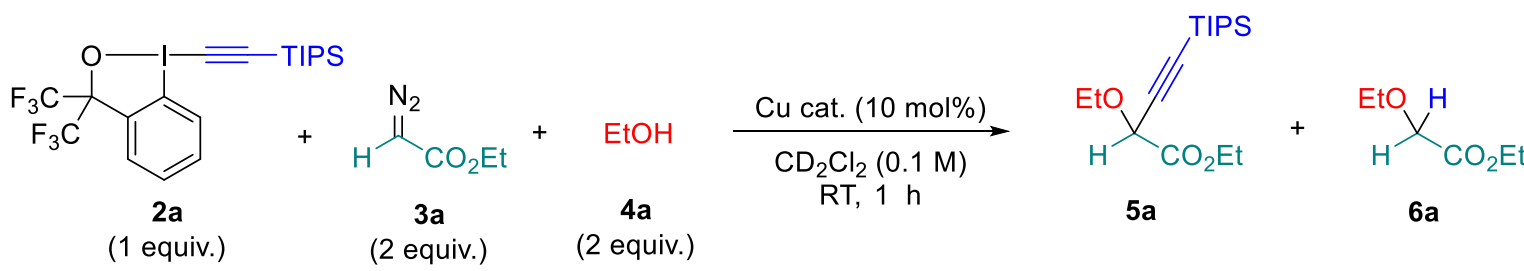

\begin{tabular}{ccc}
\hline entry & Cu cat. & $\begin{array}{c}\text { yield } 5 \mathrm{a} / 6 \mathbf{a} \\
{[\%]^{\mathrm{a}}}\end{array}$ \\
\hline 1 & $\mathrm{Cu}\left(\mathrm{CH}_{3} \mathrm{CN}_{4} \mathrm{PF}_{6}\right.$ & $74 / 18$ \\
2 & $\mathrm{CuOTf} \cdot$ toluene & $60 / 33$ \\
3 & $\mathrm{Cu}(\mathrm{OTf})_{2}$ & $47 / 11$ \\
4 & $\mathrm{CuCl}_{2}$ & $30 / 17$ \\
5 & $\mathrm{CuBr}$ & $16 / 24$ \\
6 & $\mathrm{Cul}$ & no reaction \\
7 & $\mathrm{CuTC}$ & $10 / 40$ \\
8 & $\mathrm{CuCN}$ & no reaction \\
9 & $\mathrm{CuOAc}$ & no reaction \\
10 & $\mathrm{Cu}\left(\mathrm{C}_{5} \mathrm{H}_{4} \mathrm{~F}_{3} \mathrm{O}_{2}\right)_{2}$ & no reaction \\
11 & $\mathrm{Cu}(\mathrm{OAc})_{2}$ & no reaction
\end{tabular}

[a] Determined by ${ }^{1} \mathrm{H}$ NMR analysis

It was found that $\mathrm{Cu}\left(\mathrm{CH}_{3} \mathrm{CN}\right)_{4} \mathrm{PF}_{6}$ was superior to $\mathrm{Cu}\left(\mathrm{CH}_{3} \mathrm{CN}\right)_{4} \mathrm{BF}_{4}$ to catalyze the formation of the threecomponent product $\mathbf{5 a}$.

d) Fine-tuning of the last parameters

In a $\mathrm{N}_{2}$ filled glovebox, an oven-dried $10 \mathrm{~mL}$ microwave vial was charged with $\mathrm{Cu}\left(\mathrm{CH}_{4} \mathrm{CN}\right)_{4} \mathrm{PF}_{6}(2.52 \mathrm{mg}$, $8.00 \mu \mathrm{mol}, 0.10$ equiv.) and TIPS-EBX 2a (44.0 mg, $80.0 \mu \mathrm{mol}, 1.00$ equiv.). The vial was capped, removed from the glovebox and $\mathrm{CD}_{2} \mathrm{Cl}_{2}(0.80 \mathrm{~mL}$ ) was added, followed by $\mathrm{EtOH}$ (4a) (w equiv.) and ethyl diazoacetate (3a) ( $v$ equiv.). The resulting reaction mixture was stirred at room temperature for $1 \mathrm{~h}$. After this time, ${ }^{1} \mathrm{H}$ NMR spectrum of the reaction mixture was recorded. 


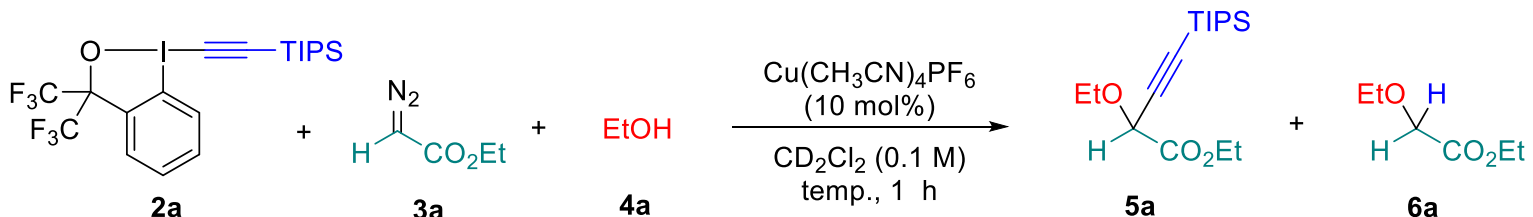
(1 equiv.)

\begin{tabular}{ccccc}
\hline entry & $\begin{array}{c}\text { EDA 3a } \\
(\text { v equiv. })\end{array}$ & $\begin{array}{c}\text { EtOH 4a } \\
(\text { w equiv. })\end{array}$ & $\begin{array}{c}\text { temp. } \\
\left({ }^{\circ} \mathrm{C}\right)\end{array}$ & $\begin{array}{c}\text { yield 5a/6a } \\
{[\%]^{\mathrm{a}}}\end{array}$ \\
\hline 1 & 2 & 4 & 25 & $94(84)^{\mathrm{b}} / 11$ \\
2 & 2 & 4 & 0 & $95 / 11$ \\
$3^{\mathrm{c}}$ & 2 & 4 & 25 & $94^{\mathrm{b} / \mathrm{n} . \mathrm{d} .}$ \\
4 & 1 & 1 & 25 & $37 / 17$ \\
\hline
\end{tabular}

[a] Determined by ${ }^{1} \mathrm{H}$ NMR analysis [b] Isolated yield [c] The diazo compounds was added as a $0.6 \mathrm{M}$ solution in DCM in $1 \mathrm{~h}$ via seringe pump. Reaction perfomed on scope scale $(2 \mathrm{a}, 0.3 \mathrm{mmol})$

It was found that 4 equivalents of EtOH 4 a was beneficial for the formation of the three-component product $\mathbf{5 a}$. It was found that the slow addition of a diluted solution of EDA $\mathbf{3} \mathbf{a}$ improved the yield of 5a. 
3. 3-component reaction of alcohols, diazo compounds and EBX reagents

General procedure A: Three-component reaction at $25^{\circ} \mathrm{C}$.

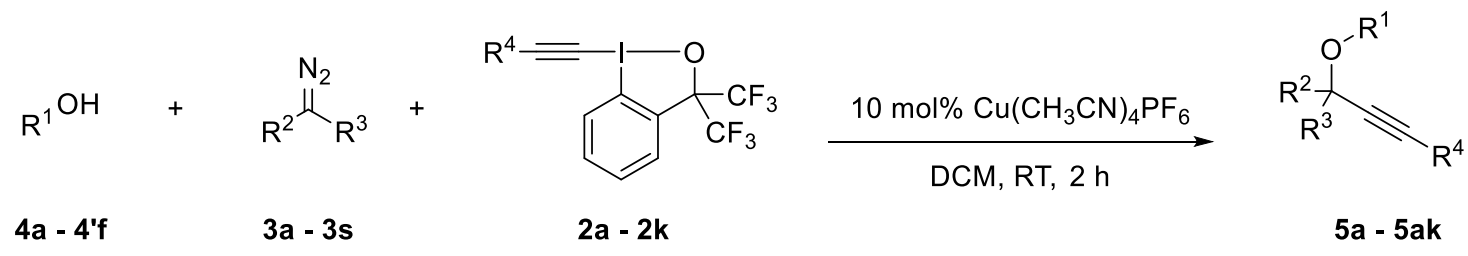

In a $\mathrm{N}_{2}$ filled glovebox, an oven-dried $10 \mathrm{~mL}$ microwave vial was charged with $\mathrm{Cu}\left(\mathrm{CH}_{3} \mathrm{CN}\right){ }_{4} \mathrm{PF}_{6}(11.2 \mathrm{mg}$, $30.0 \mu \mathrm{mol}, 0.10$ equiv.), EBX reagent ( $2 \mathrm{a}-\mathbf{2 k})(0.30 \mathrm{mmol}, 1.00$ equiv.) and alcohol (4a-4af) (1.20 mmol, 4.00 equiv.), if solid. The vial was capped, removed from the glovebox and dry DCM $(2.0 \mathrm{~mL})$ was added. The alcohol was added at this point if liquid. To the resulting solution was added a $0.6 \mathrm{M}$ solution of diazo compound ( $3 a-3 s)(0.60 \mathrm{mmol}, 2.00$ equiv.) in dry DCM in $1 \mathrm{~h}$ via seringe pump at $25{ }^{\circ} \mathrm{C}$. The system was mainted isobaric with a filled balloon with $\mathrm{N}_{2}$. At the end of the addition, the reaction was continued for $1 \mathrm{~h}$ at the same temperature. The solvent was removed under reduced pressure and the resulting crude product was purified by column chromatography using EtOAc/pentane as eluent (the solvent ratio indicated in the Rfmeasurement was used), directly without further work-up to afford the corresponding product (5a-5ak).

General procedure B: Three-component reaction at $40^{\circ} \mathrm{C}$.

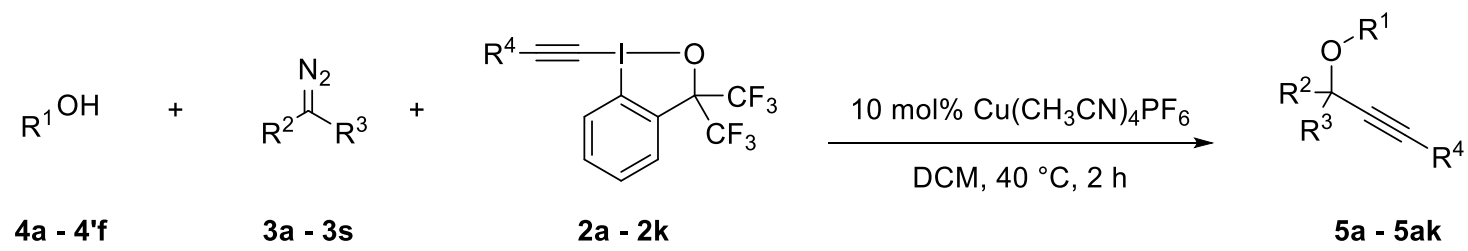

In a $\mathrm{N}_{2}$ filled glovebox, an oven-dried $10 \mathrm{~mL}$ microwave vial was charged with $\mathrm{Cu}\left(\mathrm{CH}_{3} \mathrm{CN}\right)_{4} \mathrm{PF}_{6}(11.2 \mathrm{mg}$, $30.0 \mu \mathrm{mol}, 0.10$ equiv.), EBX reagent $(\mathbf{2 a}-\mathbf{2 k})(0.30 \mathrm{mmol}, 1.00$ equiv.) and alcohol (4a-4af) (1.20 $\mathrm{mmol}, 4.00$ equiv.), if solid. The vial was capped, removed from the glovebox and dry DCM $(2.0 \mathrm{~mL})$ was added. The alcohol was added at this point if liquid. To the resulting solution was added a $0.6 \mathrm{M}$ solution of diazo compound (3a-3s) $(0.60 \mathrm{mmol}, 2.00$ equiv.) in dry DCM in $1 \mathrm{~h}$ via seringe pump at $40{ }^{\circ} \mathrm{C}$. The system was mainted isobaric with a filled balloon with $\mathrm{N}_{2}$. At the end of the addition, the reaction was continued for $1 \mathrm{~h}$ at the same temperature. The solvent was removed under reduced pressure and the resulting crude product was purified by column chromatography using EtOAc/pentane as eluent (the solvent ratio indicated in the Rf measurement was used), directly without further work-up to afford the corresponding product (5a-5ak).

Ethyl 2-ethoxy-4-(triisopropylsilyl)but-3-ynoate (5a)<smiles>CCOC(=O)C(C#C[18O])OCC</smiles>

Following general procedure $A$, starting from ethanol (4a) $(70.0 \mu \mathrm{L}, 1.20 \mathrm{mmol}), \quad((3,3-$ bis(trifluoromethyl)-1 $\lambda^{3}$-benzo[d][1,2] iodaoxol-1(3H)-yl)ethynyl)triisopropylsilane (2a) (165 mg, 0.300 $\mathrm{mmol})$, and ethyl 2-diazoacetate (3a) $(1.0 \mathrm{~mL}, 0.60 \mathrm{mmol}, 0.6 \mathrm{M}$ in DCM), afforded the title compound (6a) as a colorless oil (87 mg, $0.28 \mathrm{mmol}, 93 \%) . \mathrm{R}_{\mathrm{f}}=0.29$ (EtOAc/pentane 3:97), $p$-anisaldehyde; ${ }^{1} \mathrm{H}$ $\operatorname{NMR}\left(400 \mathrm{MHz}, \mathrm{CDCl}_{3}\right) \delta 4.75(\mathrm{~s}, 1 \mathrm{H}, \mathrm{HCC} \equiv \mathrm{C}), 4.35-4.17\left(\mathrm{~m}, 2 \mathrm{H}, \mathrm{CH}_{2} \mathrm{OC}(\mathrm{O})\right), 3.79$ (dq, J = 9.1, $7.0 \mathrm{~Hz}$, $\left.1 \mathrm{H}, \mathrm{OCH}_{2} \mathrm{CH}_{3}\right), 3.65\left(\mathrm{dq}, J=9.2,7.0 \mathrm{~Hz}, 1 \mathrm{H}, \mathrm{OCH}_{2} \mathrm{CH}_{3}\right), 1.29\left(\mathrm{~m}, 6 \mathrm{H}, 2 \times \mathrm{OCH}_{2} \mathrm{CH}_{3}\right), 1.13-0.94(\mathrm{~m}, 21 \mathrm{H}$, TIPS); ${ }^{13} \mathrm{C}$ NMR $\left(101 \mathrm{MHz}, \mathrm{CDCl}_{3}\right) \delta 167.8,100.4,89.2,69.2,64.6,62.0,18.7,15.2,14.2,11.2 ; \mathrm{IR}\left(\mathrm{V}_{\max }\right.$, 
$\left.\mathrm{cm}^{-1}\right) 2943(\mathrm{~m}), 2867(\mathrm{~m}), 2175(\mathrm{w}), 1759(\mathrm{~s}), 1464(\mathrm{~m}), 1281(\mathrm{~m}), 1192(\mathrm{~m}), 1116(\mathrm{~s}), 1032(\mathrm{~s}), 882(\mathrm{~s})$, 677 (s), 661 (s); HRMS (ESI/QTOF) m/z: [M+Na] $]^{+}$Calcd. for $\mathrm{C}_{17} \mathrm{H}_{32} \mathrm{NaO}_{3} \mathrm{Si}^{+}$335.2013; Found 335.2009.

\section{Ethyl 2-(benzyloxy)-4-(triisopropylsilyl)but-3-ynoate (5b)}<smiles>CCOC(=O)C(C#C[Pb])OCc1ccccc1</smiles>

Following general procedure $A$, starting from benzyl alcohol $(4 \mathrm{~b})(124 \mu \mathrm{L}, 1.20 \mathrm{mmol}),((3,3-$ bis(trifluoromethyl)-1 $1 \lambda^{3}$-benzo[d][1,2]iodaoxol-1(3H)-yl)ethynyl)triisopropylsilane (2a) (165 mg, 0.300 $\mathrm{mmol})$, and ethyl 2-diazoacetate (3a) $(1.0 \mathrm{~mL}, 0.60 \mathrm{mmol}, 0.6 \mathrm{M}$ in DCM), afforded the title compound (5b) as a colorless oil (85 mg, $0.23 \mathrm{mmol}, 76 \%) . \mathrm{R}_{\mathrm{f}}=0.33$ (EtOAc/pentane 3:97), $p$-anisaldehyde; ${ }^{1} \mathrm{H}$ NMR (400 MHz, CDCl $) \delta 7.43-7.27(\mathrm{~m}, 5 \mathrm{H}, \mathrm{ArH}), 4.86-4.79\left(\mathrm{~m}, 1 \mathrm{H}, \mathrm{OCH}_{2} \mathrm{Ph}\right), 4.79-4.69(\mathrm{~m}, 2 \mathrm{H}$, $\mathrm{OCH}_{2} \mathrm{Ph}$ and $\left.\mathrm{HCC} \equiv \mathrm{C}\right), 4.35-4.16\left(\mathrm{~m}, 2 \mathrm{H}, \mathrm{OCH}_{2} \mathrm{CH}_{3}\right), 1.29\left(\mathrm{t}, J=7.1 \mathrm{~Hz}, 3 \mathrm{H}, \mathrm{OCH}_{2} \mathrm{CH}_{3}\right), 1.13-0.97(\mathrm{~m}$, 21H, TIPS); ${ }^{13} \mathrm{C} \mathrm{NMR}\left(101 \mathrm{MHz}, \mathrm{CDCl}_{3}\right) \delta 167.6,136.9,128.7,128.6,128.2,100.0,90.0,70.4,68.1,62.0$, 18.7, 14.2, 11.3; IR ( $\left.v_{\max }, \mathrm{cm}^{-1}\right) 2943(\mathrm{~m}), 2866(\mathrm{~m}), 2175(\mathrm{w}), 1756(\mathrm{~s}), 1463(\mathrm{~m}), 1275(\mathrm{~m}), 1192(\mathrm{~s})$, 1111 (s), 1031 (s), 882 (s), 739 (m), 697 (s), 677 (s); HRMS (ESI/QTOF) m/z: [M+Na] ${ }^{+}$Calcd. for $\mathrm{C}_{22} \mathrm{H}_{34} \mathrm{NaO}_{3} \mathrm{Si}^{+}$397.2169; Found 397.2172.

Ethyl 2-((4-bromobenzyl)oxy)-4-(triisopropylsilyl)but-3-ynoate (5c)<smiles>CCOC(=O)C(C#CC(=S)[Sn])OCc1ccc(Br)cc1</smiles>

Following general procedure A, starting from 4-bromobenzyl alcohol (4c) $(224 \mathrm{mg}, 1.20 \mathrm{mmol}),((3,3-$ bis(trifluoromethyl)-1 $\lambda^{3}$-benzo[d][1,2] iodaoxol-1(3H)-yl)ethynyl)triisopropylsilane (2a) (165 mg, 0.300 $\mathrm{mmol})$, and ethyl 2-diazoacetate (3a) $(1.0 \mathrm{~mL}, 0.60 \mathrm{mmol}, 0.6 \mathrm{M}$ in DCM), afforded the title compound (5c) as a colorless oil (99 mg, $0.22 \mathrm{mmol}, 73 \%) . \mathrm{R}_{\mathrm{f}}=0.29$ (EtOAc/pentane 3:97), $p$-anisaldehyde; ${ }^{1} \mathrm{H}$ $\operatorname{NMR}\left(400 \mathrm{MHz}, \mathrm{CDCl}_{3}\right) \delta 7.51-7.45(\mathrm{~m}, 2 \mathrm{H}, \mathrm{ArH}), 7.32-7.24(\mathrm{~m}, 2 \mathrm{H}, \mathrm{ArH}), 4.80-4.72\left(\mathrm{~m}, 2 \mathrm{H}, \mathrm{OCH}_{2} \mathrm{Ar}\right.$ and $H C C=C), 4.68\left(\mathrm{~m}, 1 \mathrm{H}, \mathrm{OCH}_{2} \mathrm{Ar}\right), 4.35-4.16\left(\mathrm{~m}, 2 \mathrm{H}, \mathrm{OCH}_{2} \mathrm{CH}_{3}\right), 1.29\left(\mathrm{t}, J=7.1 \mathrm{~Hz}, 3 \mathrm{H}, \mathrm{OCH}_{2} \mathrm{CH}_{3}\right), 1.12$ $-0.96(\mathrm{~m}, 21 \mathrm{H}, \mathrm{TIPS}) ;{ }^{13} \mathrm{C}$ NMR $\left(101 \mathrm{MHz}, \mathrm{CDCl}_{3}\right) \delta 167.5,136.1,131.7,130.2,122.2,99.7,90.4,69.6$, 68.2, 62.1, 18.7, 14.2, 11.2; IR ( $\left.\mathrm{v}_{\max }, \mathrm{cm}^{-1}\right) 2943(\mathrm{~m}), 2892(\mathrm{~m}), 2866(\mathrm{~m}), 1755(\mathrm{~s}), 1489(\mathrm{~m}), 1463(\mathrm{~m})$, $1367(\mathrm{~m}), 1279(\mathrm{~m}), 1194(\mathrm{~s}), 1112(\mathrm{~s}), 1070(\mathrm{~s}), 1039(\mathrm{~s}), 1012(\mathrm{~s}), 882(\mathrm{~s}), 801(\mathrm{~s}), 677(\mathrm{~s})$; HRMS (ESI/QTOF) m/z: [M+Na] $]^{+}$Calcd. for $\mathrm{C}_{22} \mathrm{H}_{33} \mathrm{BrNaO}_{3} \mathrm{Si}^{+}$475.1275; Found 475.1279.

\section{Ethyl 2-(furan-2-ylmethoxy)-4-(triisopropylsilyl)but-3-ynoate (5d)}<smiles>CCOC(=O)C(C#CC(=O)[Sn])OCc1ccco1</smiles>

Following general procedure A, starting from furfuryl alcohol (4d) $(104 \mu \mathrm{L}, 1.20 \mathrm{mmol}),((3,3-$ bis(trifluoromethyl)-1 $\lambda^{3}$-benzo[d][1,2] iodaoxol-1(3H)-yl)ethynyl)triisopropylsilane (2a) (165 mg, 0.300 $\mathrm{mmol})$, and ethyl 2-diazoacetate (3a) $(1.0 \mathrm{~mL}, 0.60 \mathrm{mmol}, 0.6 \mathrm{M}$ in DCM), afforded the title compound (5d) as a colorless oil $(69 \mathrm{mg}, 0.19 \mathrm{mmol}, 63 \%) . \mathrm{R}_{\mathrm{f}}=0.28$ (EtOAc/pentane 3:97), $p$-anisaldehyde; ${ }^{1} \mathrm{H}$ $\operatorname{NMR}\left(400 \mathrm{MHz}, \mathrm{CDCl}_{3}\right) \delta 7.42$ (dd, $\left.J=1.9,0.8 \mathrm{~Hz}, 1 \mathrm{H}, \mathrm{ArH}\right), 6.39$ (dd, $\left.J=3.2,0.8 \mathrm{~Hz}, 1 \mathrm{H}, \mathrm{ArH}\right), 6.34$ (dd, $J=3.2,1.9 \mathrm{~Hz}, 1 \mathrm{H}, \mathrm{ArH}), 4.81-4.67\left(\mathrm{~m}, 3 \mathrm{H}, \mathrm{HCC} \equiv \mathrm{C}\right.$ and $\left.\mathrm{CH}_{2} \mathrm{Ar}\right), 4.33-4.16\left(\mathrm{~m}, 2 \mathrm{H}, \mathrm{OCH}_{2} \mathrm{CH}_{3}\right), 1.29(\mathrm{t}$, $\left.J=7.1 \mathrm{~Hz}, 3 \mathrm{H}, \mathrm{OCH}_{2} \mathrm{CH}_{3}\right), 1.12-0.96(\mathrm{~m}, 21 \mathrm{H}, \mathrm{TIPS}) ;{ }^{13} \mathrm{C} \mathrm{NMR}\left(101 \mathrm{MHz}, \mathrm{CDCl}_{3}\right) \delta 167.4,150.5,143.4$, 110.8, 110.5, 99.6, 90.4, 67.8, 62.1, 61.9, 18.7, 14.2, 11.3; IR $\left(v_{\max }, \mathrm{cm}^{-1}\right) 2943(\mathrm{~s}), 2866$ (s), $2174(\mathrm{w})$, 
$1756(\mathrm{~s}), 1464(\mathrm{~m}), 1277(\mathrm{~m}), 1193(\mathrm{~s}), 1151$ (s), $1097(\mathrm{~s}), 1043$ (s), 1015 (s), $921(\mathrm{~m}), 883$ (s), 737 (s), 676 (s); HRMS (ESI/QTOF) m/z: [M+Na] ${ }^{+}$Calcd. for $\mathrm{C}_{20} \mathrm{H}_{32} \mathrm{NaO}_{4} \mathrm{Si}^{+}$387.1962; Found 387.1974.

\section{Ethyl 2-(cyclohexyloxy)-4-(triisopropylsilyl)but-3-ynoate (5e)}

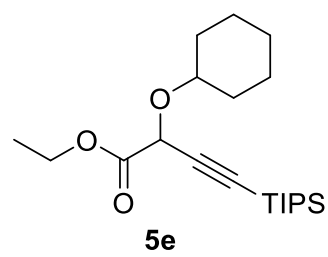

Following general procedure A, starting from cyclohexanol (4e) $(127 \mu \mathrm{L}, 1.20 \mathrm{mmol}),((3,3-$ bis(trifluoromethyl)-1 $\lambda^{3}$-benzo[d][1,2]iodaoxol-1(3H)-yl)ethynyl)triisopropylsilane (2a) (165 mg, 0.300 $\mathrm{mmol})$, and ethyl 2-diazoacetate (3a) $(1.0 \mathrm{~mL}, 0.60 \mathrm{mmol}, 0.6 \mathrm{M}$ in DCM), afforded the title compound (5e) as a colorless oil (99 mg, $0.27 \mathrm{mmol}, 90 \%) . \mathrm{R}_{\mathrm{f}}=0.33$ (EtOAc/pentane 3:97), $p$-anisaldehyde; ${ }^{1} \mathrm{H}$ $\operatorname{NMR}\left(400 \mathrm{MHz}, \mathrm{CDCl}_{3}\right) \delta 4.82(\mathrm{~s}, 1 \mathrm{H}, \mathrm{HCC} \equiv \mathrm{C}), 4.36-4.15\left(\mathrm{~m}, 2 \mathrm{H}, \mathrm{OCH}_{2} \mathrm{CH}_{3}\right), 3.65(\mathrm{tt}, J=9.6,3.9 \mathrm{~Hz}, 1 \mathrm{H}$, $\mathrm{O}-\mathrm{CH}$-cyclohexyl), $2.04-1.90(\mathrm{~m}, 2 \mathrm{H}, 2 \times \mathrm{CH}$-cyclohexyl), $1.82-1.69(\mathrm{~m}, 2 \mathrm{H}, 2 \times \mathrm{CH}$-cyclohexyl), $1.56-1.12(\mathrm{~m}$, $9 \mathrm{H}, 2 \times \mathrm{CH}_{2}$-cyclohexyl and $\left.\mathrm{OCH}_{2} \mathrm{CH}_{3}\right), 1.11-0.93(\mathrm{~m}, 21 \mathrm{H}, \mathrm{TIPS}) ;{ }^{13} \mathrm{C} \mathrm{NMR}\left(101 \mathrm{MHz}, \mathrm{CDCl}_{3}\right) \delta 168.2,101.3$, 88.6, 77.1, 67.2, 61.9, 32.9, 31.9, 25.8, 24.5, 24.3, 18.7, 14.2, 11.3; IR $\left(v_{\max }, \mathrm{cm}^{-1}\right) 2943(\mathrm{~m}), 2866(\mathrm{~m})$, $2175(\mathrm{w}), 1756(\mathrm{~s}), 1463(\mathrm{~m}), 1275(\mathrm{~m}), 1192(\mathrm{~s}), 1111(\mathrm{~s}), 1040(\mathrm{~s}), 882(\mathrm{~s}), 739(\mathrm{~m}), 697(\mathrm{~s}), 676(\mathrm{~s})$; HRMS (ESI/QTOF) m/z: [M+Na] $]^{+}$Calcd. for $\mathrm{C}_{21} \mathrm{H}_{38} \mathrm{NaO}_{3} \mathrm{Si}^{+}$389.2482; Found 389.2487.

\section{Ethyl 2-((1,3-difluoropropan-2-yl)oxy)-4-(triisopropylsilyl)but-3-ynoate (5f)}<smiles>CCOC(=O)C(C#CC(=O)I)OC(C)F</smiles>

Following general procedure A, starting from 1,2-difluoro-2-propanol (4f) $(93.0 \mu \mathrm{L}, 1.20 \mathrm{mmol}),((3,3-$ bis(trifluoromethyl)-1 $\lambda^{3}$-benzo[d][1,2] iodaoxol-1(3H)-yl)ethynyl)triisopropylsilane (2a) (165 mg, 0.300 $\mathrm{mmol})$, and ethyl 2-diazoacetate (3a) $(1.0 \mathrm{~mL}, 0.60 \mathrm{mmol}, 0.6 \mathrm{M}$ in DCM), afforded the title compound (5f) as a colorless oil ( $68 \mathrm{mg}, 0.19 \mathrm{mmol}, 63 \%) . \mathrm{R}_{\mathrm{f}}=0.27$ (EtOAc/pentane 3:97), $p$-anisaldehyde; ${ }^{1} \mathrm{H}$ NMR $\left(400 \mathrm{MHz}, \mathrm{CDCl}_{3}\right) \delta 4.99(\mathrm{~s}, 1 \mathrm{H}, \mathrm{HCC} \equiv \mathrm{C}), 4.78-4.64\left(\mathrm{~m}, 2 \mathrm{H}, 2 \times \mathrm{CH}_{2} \mathrm{~F}\right), 4.64-4.52\left(\mathrm{~m}, 2 \mathrm{H}, 2 \times \mathrm{CH}_{2} \mathrm{~F}\right)$, $4.36-4.17\left(\mathrm{~m}, 3 \mathrm{H}, \mathrm{OCH}\right.$ and $\left.\mathrm{OCH}_{2} \mathrm{CH}_{3}\right), 1.30\left(\mathrm{t}, J=7.1 \mathrm{~Hz}, 3 \mathrm{H}, \mathrm{OCH}_{2} \mathrm{CH}_{3}\right), 1.12-0.95(\mathrm{~m}, 21 \mathrm{H}, \mathrm{TIPS}) ;{ }^{13} \mathrm{C}$ $\operatorname{NMR}\left(101 \mathrm{MHz}, \mathrm{CDCl}_{3}\right) \delta 167.4,99.4,90.9,81.5$ (ddd, $\left.J=171.5,15.9,6.5 \mathrm{~Hz}\right), 74.6(\mathrm{t}, J=20.6 \mathrm{~Hz}), 69.2$, 62.2, 18.6, 14.2, 11.2; $\left.{ }^{19} \mathrm{~F} \mathrm{NMR} \mathrm{(376} \mathrm{MHz,} \mathrm{CDCl} 3\right) \delta-76.2$; IR $\left(v_{\max }, \mathrm{cm}^{-1}\right) 2945(\mathrm{~m}), 2894(\mathrm{~m}), 2867(\mathrm{~m})$, $2176(\mathrm{w}), 1755(\mathrm{~s}), 1464(\mathrm{~m}), 1282(\mathrm{~m}), 1200(\mathrm{~s}), 1121(\mathrm{~s}), 1091(\mathrm{~s}), 1028(\mathrm{~s}), 970(\mathrm{~m}), 882(\mathrm{~s}), 677(\mathrm{~s})$, 660 (s); HRMS (ESI/QTOF) m/z: [M+Na] Calcd. for $\mathrm{C}_{18} \mathrm{H}_{32} \mathrm{~F}_{2} \mathrm{NaO}_{3} \mathrm{Si}^{+}$385.1981; Found 385.1987.

\section{Ethyl 2-(tert-butoxy)-4-(triisopropylsilyl)but-3-ynoate (5g)}

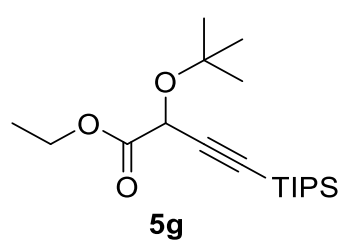

Following general procedure $A$, starting from tert-butanol $(\mathbf{4 g})(115 \mu \mathrm{L}, 1.20 \mathrm{mmol}),((3,3-$ bis(trifluoromethyl)-1 $1 \lambda^{3}$-benzo[d][1,2]iodaoxol-1(3H)-yl)ethynyl)triisopropylsilane (2a) (165 mg, 0.300 $\mathrm{mmol})$, and ethyl 2-diazoacetate (3a) $(1.0 \mathrm{~mL}, 0.60 \mathrm{mmol}, 0.6 \mathrm{M}$ in DCM), afforded the title compound $(5 \mathrm{~g})$ as a colorless oil $(70 \mathrm{mg}, 0.21 \mathrm{mmol}, 69 \%) . \mathrm{R}_{\mathrm{f}}=0.40$ (EtOAc/pentane 3:97), $p$-anisaldehyde; ${ }^{1} \mathrm{H}$ $\operatorname{NMR}\left(400 \mathrm{MHz}, \mathrm{CDCl}_{3}\right) \delta 4.76(\mathrm{~s}, 1 \mathrm{H}, \mathrm{HCC} \equiv \mathrm{C}), 4.34-4.15\left(\mathrm{~m}, 2 \mathrm{H}, \mathrm{OCH}_{2} \mathrm{CH}_{3}\right), 1.31-1.26(\mathrm{~m}, 12 \mathrm{H}, t \mathrm{Bu}$ 
and $\left.\mathrm{OCH}_{2} \mathrm{CH}_{3}\right), 1.10-0.93(\mathrm{~m}, 21 \mathrm{H}, \mathrm{TIPS}) ;{ }^{13} \mathrm{C} \mathrm{NMR}\left(101 \mathrm{MHz}, \mathrm{CDCl}_{3}\right) \delta 168.8,103.6,87.3,76.5,63.2$, 61.9, 28.1, 18.7, 14.2, 11.3; IR ( $\left.\mathrm{v}_{\max }, \mathrm{cm}^{-1}\right) 2961(\mathrm{~m}), 2943(\mathrm{~m}), 2866(\mathrm{~m}), 2177(\mathrm{w}), 1766(\mathrm{~s}), 1741(\mathrm{~m})$, $1464(\mathrm{~m}), 1367(\mathrm{~s}), 1275(\mathrm{~m}), 1254(\mathrm{~m}), 1188(\mathrm{~s}), 1096(\mathrm{~s}), 1033(\mathrm{~s}), 882(\mathrm{~s}), 750(\mathrm{~m}), 677$ (s); HRMS (ESI/QTOF) m/z: [M+Na] ${ }^{+}$Calcd. for $\mathrm{C}_{19} \mathrm{H}_{36} \mathrm{NaO}_{3} \mathrm{Si}^{+}$363.2326; Found 363.2329.

\section{Ethyl 2-(allyloxy)-4-(triisopropylsilyl)but-3-ynoate (5h)}<smiles>C=CCOC(C#C[In])C(=O)OCC</smiles>

Following general procedure $B$, starting from allyl alcohol $(4 \mathbf{h})(115 \mu \mathrm{L}, 1.20 \mathrm{mmol}),((3,3-$ bis(trifluoromethyl)-1 $\lambda^{3}$-benzo[d][1,2]iodaoxol-1(3H)-yl)ethynyl)triisopropylsilane (2a) (165 mg, 0.300 $\mathrm{mmol})$, and ethyl 2-diazoacetate (3a) $(1.0 \mathrm{~mL}, 0.60 \mathrm{mmol}, 0.6 \mathrm{M}$ in DCM), afforded the title compound (5h) as a colorless oil (45 mg, $0.14 \mathrm{mmol}, 46 \%) . \mathrm{R}_{\mathrm{f}}=0.31$ (EtOAc/pentane 3:97), $p$-anisaldehyde; ${ }^{1} \mathrm{H}$ $\operatorname{NMR}\left(400 \mathrm{MHz}, \mathrm{CDCl}_{3}\right) \delta 5.94$ (dddd, $\left.J=17.0,10.3,6.5,5.4 \mathrm{~Hz}, 1 \mathrm{H}, \mathrm{CH}=\mathrm{CH}_{2}\right), 5.34$ (dq, $J=17.2,1.6 \mathrm{~Hz}$, $\left.1 \mathrm{H}, \mathrm{CH}=\mathrm{CH}_{2}\right), 5.25\left(\mathrm{dq}, J=10.4,1.2 \mathrm{~Hz}, 1 \mathrm{H}, \mathrm{CH}=\mathrm{CH}_{2}\right), 4.79(\mathrm{~s}, 1 \mathrm{H}, \mathrm{HCC} \equiv \mathrm{C}), 4.36-4.12\left(\mathrm{~m}, 4 \mathrm{H}, \mathrm{OCH}_{2} \mathrm{CH}\right.$ and $\left.\mathrm{OCH}_{2} \mathrm{CH}_{3}\right), 1.30$ (t, $\left.J=7.1 \mathrm{~Hz}, 3 \mathrm{H}, \mathrm{OCH}_{2} \mathrm{CH}_{3}\right), 1.12-0.94(\mathrm{~m}, 21 \mathrm{H}, \mathrm{TIPS}) ;{ }^{13} \mathrm{C} \mathrm{NMR}\left(101 \mathrm{MHz}, \mathrm{CDCl}_{3}\right)$ $\delta$ 167.6, 133.6, 118.9, 100.1, 89.7, 69.7, 68.2, 62.0, 18.7, 14.2, 11.2; IR $\left(v_{\max }, \mathrm{cm}^{-1}\right) 2943(\mathrm{~m}), 2866(\mathrm{~s})$, $2175(\mathrm{w}), 1758(\mathrm{~s}), 1464(\mathrm{~m}), 1270(\mathrm{~m}), 1189(\mathrm{~s}), 1111(\mathrm{~s}), 1038(\mathrm{~s}), 996(\mathrm{~s}), 921(\mathrm{~m}), 882(\mathrm{~s}), 676(\mathrm{~s})$; HRMS (ESI/QTOF) m/z: [M+Na] $]^{+}$Calcd. for $\mathrm{C}_{18} \mathrm{H}_{32} \mathrm{NaO}_{3} \mathrm{Si}^{+}$347.2013; Found 347.2008.

\section{Ethyl 2-((2-methyl-4-phenylbut-3-yn-2-yl)oxy)-4-(triisopropylsilyl)but-3-ynoate (5i)}

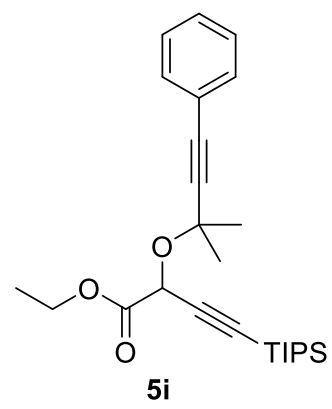

Following general procedure $B$, starting from 4-phenyl-2-methyl-3-butyn-2-ol (4i) (192 mg, $1.20 \mathrm{mmol}$ ), ((3,3-bis(trifluoromethyl)-1 $\lambda^{3}$-benzo[d][1,2]iodaoxol-1(3H)-yl)ethynyl)triisopropylsilane (2a) (165 mg, $0.300 \mathrm{mmol}$ ), and ethyl 2-diazoacetate (3a) $(1.0 \mathrm{~mL}, 0.60 \mathrm{mmol}, 0.6 \mathrm{M}$ in DCM), afforded the title compound (5i) as a colorless oil (54 mg, $0.13 \mathrm{mmol}, 42 \%) . \mathrm{R}_{\mathrm{f}}=0.38$ (EtOAc/pentane 3:97), $p$ anisaldehyde; ${ }^{1} \mathrm{H}$ NMR (400 MHz, $\left.\mathrm{CDCl}_{3}\right) \delta 7.45-7.38(\mathrm{~m}, 2 \mathrm{H}, \mathrm{ArH}), 7.33-7.27(\mathrm{~m}, 3 \mathrm{H}, \mathrm{ArH}), 5.20(\mathrm{~s}$, $1 \mathrm{H}, \mathrm{HCC} \equiv \mathrm{C}), 4.35-4.12\left(\mathrm{~m}, 2 \mathrm{H}, \mathrm{OCH}_{2} \mathrm{CH}_{3}\right), 1.68\left(\mathrm{~s}, 3 \mathrm{H}, \mathrm{C}\left(\mathrm{CH}_{3}\right)_{2}\right), 1.62\left(\mathrm{~s}, 3 \mathrm{H}, \mathrm{C}\left(\mathrm{CH}_{3}\right)_{2}\right), 1.26(\mathrm{t}, J=7.1 \mathrm{~Hz}$, $\left.3 \mathrm{H}, \mathrm{OCH}_{2} \mathrm{CH}_{3}\right), 1.09-0.93(\mathrm{~m}, 21 \mathrm{H}, \mathrm{TIPS}) ;{ }^{13} \mathrm{C} \mathrm{NMR}\left(101 \mathrm{MHz}, \mathrm{CDCl}_{3}\right) \delta 168.5,131.9,128.6,128.4,122.5$, 102.8, 90.1, 88.0, 85.8, 73.7, 65.4, 61.9, 30.0, 29.4, 18.7, 14.2, 11.3; IR $\left(v_{\max }, \mathrm{cm}^{-1}\right) 2943(\mathrm{~m}), 2866(\mathrm{~m})$, $2178(\mathrm{w}), 1765(\mathrm{~s}), 1741(\mathrm{~m}), 1464(\mathrm{~m}), 1283(\mathrm{~m}), 1269(\mathrm{~m}), 1184(\mathrm{~s}), 1152(\mathrm{~s}), 1091(\mathrm{~s}), 1038(\mathrm{~s}), 882$ (s), 756 (s), 690 (s), 677 (s), 661 (s); HRMS (ESI/QTOF) m/z: [M+Na] ${ }^{+}$Calcd. for $\mathrm{C}_{26} \mathrm{H}_{38} \mathrm{NaO}_{3} \mathrm{Si}^{+}$449.2482; Found 449.2485 . 
Ethyl 2-hydroxy-4-(triisopropylsilyl)but-3-ynoate (5j)

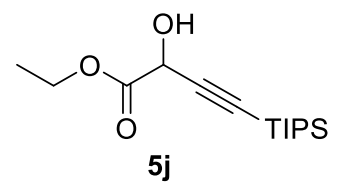

Adapted from general procedure A, starting from water $(4 \mathbf{j})(54 \mu \mathrm{L}, 3.0 \mathrm{mmol}, 10.0$ equiv.), ((3,3bis(trifluoromethyl)-1 $\lambda^{3}$-benzo[d][1,2]iodaoxol-1(3H)-yl)ethynyl)triisopropylsilane (2a) (165 mg, 0.300 mmol, 1.00 equiv.), and ethyl 2-diazoacetate (3a) $(1.0 \mathrm{~mL}, 0.60 \mathrm{mmol}, 0.6 \mathrm{M}$ in DCM, 2.00 equiv.), afforded the title compound $(\mathbf{5 j})$ as a colorless oil $(28 \mathrm{mg}, 0.10 \mathrm{mmol}, 33 \%) . \mathrm{R}_{\mathrm{f}}=0.11$ (EtOAc/pentane 3:97), $p$-anisaldehyde; ${ }^{1} \mathrm{H}$ NMR $\left(400 \mathrm{MHz}, \mathrm{CDCl}_{3}\right) \delta 4.85(\mathrm{~d}, J=5.2 \mathrm{~Hz}, 1 \mathrm{H}, \mathrm{HCC} \equiv \mathrm{C}), 4.41-4.20(\mathrm{~m}, 2 \mathrm{H}$, $\mathrm{OCH}_{2} \mathrm{CH}_{3}$ ), 3.00 (br d, $\left.J=7.0 \mathrm{~Hz}, 1 \mathrm{H}, \mathrm{OH}\right), 1.32\left(\mathrm{t}, J=7.1 \mathrm{~Hz}, 3 \mathrm{H}, \mathrm{OCH}_{2} \mathrm{CH}_{3}\right), 1.09-1.02(\mathrm{~m}, 21 \mathrm{H}, \mathrm{TIPS})$; ${ }^{13} \mathrm{C} \mathrm{NMR}\left(101 \mathrm{MHz}, \mathrm{CDCl}_{3}\right) \delta 170.6,102.3,87.4,62.8,62.1,18.6,14.2,11.2 ; \mathrm{IR}\left(\mathrm{v}_{\max }, \mathrm{cm}^{-1}\right) 3469(\mathrm{br} \mathrm{w})$, $2943(\mathrm{~s}), 2866$ (s), $2177(\mathrm{w}), 2099(\mathrm{~m}), 1745(\mathrm{~s}), 1465(\mathrm{~m}), 1301(\mathrm{~m}), 1259(\mathrm{~m}), 1202(\mathrm{~m}), 1093(\mathrm{~s}), 1028$ (s), $882(\mathrm{~s}), 677$ (s), 661 (s); HRMS (ESI/QTOF) m/z: [M+Na] ${ }^{+}$Calcd. for $\mathrm{C}_{15} \mathrm{H}_{28} \mathrm{NaO}_{3} \mathrm{Si}^{+}$307.1700; Found 307.1700 .

\section{Ethyl 2-(((1R,2S,5R)-2-isopropyl-5-methylcyclohexyl)oxy)-4-(triisopropylsilyl)but-3-ynoate (5k)}<smiles>CCOC(=O)C(C#CC(C)C)OC1C[C@H](C)CC[C@H]1C(C)C</smiles>

Following general procedure A, starting from (-)-menthol (4k) (188 mg, $1.20 \mathrm{mmol}),((3,3-$ bis(trifluoromethyl)-1 $\lambda^{3}$-benzo[d][1,2] iodaoxol-1(3H)-yl)ethynyl)triisopropylsilane (2a) (165 mg, 0.300 $\mathrm{mmol})$, and ethyl 2-diazoacetate (3a) $(1.0 \mathrm{~mL}, 0.60 \mathrm{mmol}, 0.6 \mathrm{M}$ in DCM), afforded the title compound (5k) (50:50 dr in the crude ${ }^{1} \mathrm{H}$ NMR) as a colorless oil $(107 \mathrm{mg}, 0.25 \mathrm{mmol}, 84 \%)$. A pure analytical sample of each diastereoisomer was isolated by PTLC using EtOAc/pentane 4:96 as eluent. Diaster 5k: $\mathrm{R}_{\mathrm{f}}=0.35$ (EtOAc/pentane 3:97), $p$-anisaldehyde; ${ }^{1} \mathrm{H}$ NMR $\left(400 \mathrm{MHz}, \mathrm{CDCl}_{3}\right) \delta 4.82(\mathrm{~s}, 1 \mathrm{H}, \mathrm{HCC} \equiv \mathrm{C}), 4.36$ - $4.12\left(\mathrm{~m}, 2 \mathrm{H}, \mathrm{OCH}_{2} \mathrm{CH}_{3}\right), 3.51(\mathrm{td}, J=10.6,4.2 \mathrm{~Hz}, 1 \mathrm{H}, \mathrm{OCH}$-cyclohexyl), 2.31 (pd, J = 7.0, $2.8 \mathrm{~Hz}, 1 \mathrm{H}, \mathrm{CH}-$ cyclohexyl), $2.09-2.00(\mathrm{~m}, 1 \mathrm{H}, \mathrm{CH}$-cyclohexyl), $1.70-1.58(\mathrm{~m}, 2 \mathrm{H}, \mathrm{CH}$-cyclohexyl), $1.40-1.24(\mathrm{~m}, 6 \mathrm{H}, \mathrm{CH}$-isopropyl,

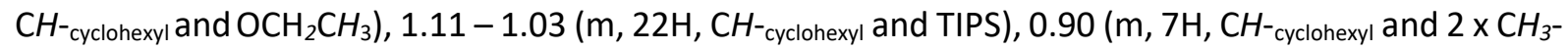
isopropyl), $0.82\left(\mathrm{~d}, J=7.0 \mathrm{~Hz}, 3 \mathrm{H}, \mathrm{CH}_{3}{ }^{-}\right.$methyl); ${ }^{13} \mathrm{C} \mathrm{NMR}\left(101 \mathrm{MHz}, \mathrm{CDCl}_{3}\right) \delta 168.1,101.0,88.8,78.4,67.1$, 61.9, 47.8, 39.8, 34.6, 31.7, 25.7, 23.7, 22.4, 21.0, 18.7, 16.6, 14.2, 11.3; Diaster $\mathbf{5 k}^{\prime}: \mathrm{R}_{\mathrm{f}}=0.36$ (EtOAc/pentane 3:97), $p$-anisaldehyde; ${ }^{1} \mathrm{H}$ NMR $\left(400 \mathrm{MHz}, \mathrm{CDCl}_{3}\right) \delta 4.56(\mathrm{~s}, 1 \mathrm{H}, \mathrm{HCC} \equiv \mathrm{C}), 4.16-4.00(\mathrm{~m}$, $\left.2 \mathrm{H}, \mathrm{OCH}_{2} \mathrm{CH}_{3}\right), 3.21(\mathrm{td}, \mathrm{J}=10.6,4.4 \mathrm{~Hz}, 1 \mathrm{H}, \mathrm{OCH}$-cyclohexy) $) 2.10(\mathrm{~m}, 2 \mathrm{H}, \mathrm{CH}$-cyclohexyl), $1.51-1.42(\mathrm{~m}, 2 \mathrm{H}$, $\mathrm{CH}$-cyclohexyl), $1.24-1.05$ (m, 6H, CH-isopropyl, $\mathrm{CH}$-cyclohexyl and $\left.\mathrm{OCH}_{2} \mathrm{CH}_{3}\right), 0.90$ (s, 22H, $\mathrm{CH}$-cyclohexyl and TIPS),

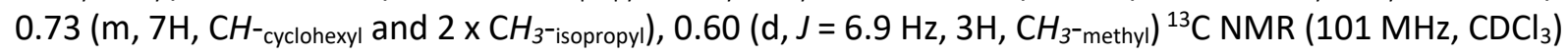
$\delta 168.1,102.1,88.6,80.9,69.1,61.9,48.3,41.7,34.4,31.8,25.5,23.4,22.3,21.1,18.7,16.3,14.2$, 11.3; IR ( $\left.v_{\max }, \mathrm{cm}^{-1}\right) 2952(\mathrm{~s}), 2925(\mathrm{~s}), 2867(\mathrm{~s}), 1766(\mathrm{~s}), 1744(\mathrm{~m}), 1463(\mathrm{~m}), 1367(\mathrm{~m}), 1274(\mathrm{~m}), 1186$ (s), 1107 (s), 1038 (s), 882 (s), 677 (s); HRMS (ESI/QTOF) m/z: [M+Na] $]^{+}$Calcd. for $\mathrm{C}_{25} \mathrm{H}_{46} \mathrm{NaO}_{3} \mathrm{Si}^{+}$ 445.3108; Found 445.3116. 


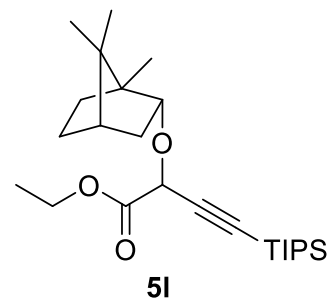

Following general procedure $A$, starting from (-)-borneol (4I) $(185 \mathrm{mg}, 1.20 \mathrm{mmol}), \quad((3,3-$ bis(trifluoromethyl)-1 $\lambda^{3}$-benzo[d][1,2]iodaoxol-1(3H)-yl)ethynyl)triisopropylsilane (2a) (165 mg, 0.300 $\mathrm{mmol})$, and ethyl 2-diazoacetate (3a) $(1.0 \mathrm{~mL}, 0.60 \mathrm{mmol}, 0.6 \mathrm{M}$ in DCM), afforded the title compound (5I) $\left(55: 45 d r\right.$ in the crude ${ }^{1} \mathrm{H}$ NMR) as a colorless oil $(110 \mathrm{mg}, 0.260 \mathrm{mmol}, 87 \%) . \mathrm{R}_{\mathrm{f}}=0.45$ (EtOAc/pentane 3:97), $p$-anisaldehyde; ${ }^{1} \mathrm{H}$ NMR (400 MHz, $\left.\mathrm{CDCl}_{3}\right) \delta 4.75$ and $4.68(2 \times \mathrm{s}, 1 \mathrm{H}, \mathrm{HCC} \equiv \mathrm{C})$, $4.33-4.15\left(\mathrm{~m}, 2 \mathrm{H}, \mathrm{OCH}_{2} \mathrm{CH}_{3}\right), 4.06-3.92(\mathrm{~m}, 1 \mathrm{H}, \mathrm{OCH}), 2.25-1.99(\mathrm{~m}, 2 \mathrm{H}, \mathrm{CH}$-bicyclo[2.2.1]heptan-2-yl), 1.77 - $1.59\left(\mathrm{~m}, 2 \mathrm{H}, \mathrm{CH}\right.$-bicyclo[2.2.1] heptan-2-yl), $1.34-1.15\left(\mathrm{~m}, 6 \mathrm{H}, \mathrm{CH}\right.$-bicyclo[2.2.1] heptan-2-yl and $\left.\mathrm{OCH}_{2} \mathrm{CH}_{3}\right), 1.12-0.94$ (m, 21H, TIPS), $0.92-0.88\left(\mathrm{~m}, 3 \mathrm{H}, \mathrm{CH}_{3}\right), 0.87-0.81\left(\mathrm{~m}, 6 \mathrm{H}, 2 \times \mathrm{CH}_{3}\right) ;{ }^{13} \mathrm{C} \mathrm{NMR}\left(101 \mathrm{MHz}, \mathrm{CDCl}_{3}\right) \delta 168.3$, $168.0,101.7,101.5,88.9,88.5,84.6,83.7,69.6,68.7,61.8,61.7,49.6,49.5,48.0,45.2,36.7,36.0,28.3$, $28.2,26.8,26.7,19.9,19.0,18.9,18.7,14.2,14.0,13.7,11.3$; Not all signals could be resolved. IR ( $v_{\max }$, $\left.\mathrm{cm}^{-1}\right) 2943(\mathrm{~m}), 2891(\mathrm{~m}), 2866(\mathrm{~m}), 2178(\mathrm{w}), 1765(\mathrm{~s}), 1741(\mathrm{~m}), 1464(\mathrm{~m}), 1269(\mathrm{~m}), 1184(\mathrm{~s}), 1152$ (s), 1091 (s), 1038 (s), 882 (s), 756 (s), 690 (s), 677 (s), 661 (s); HRMS (ESI/QTOF) m/z: [M+Na] ${ }^{+}$Calcd. for $\mathrm{C}_{25} \mathrm{H}_{44} \mathrm{NaO}_{3} \mathrm{Si}^{+}$443.2952; Found 443.2966.

\section{Ethyl 2-(( $2 R, 3 a R, 6 R, 7 R, 8 \mathrm{a} R)-2,6,8,8$-tetramethyloctahydro-1H-3a,7-methanoazulen-6-yl)oxy)-4-} (triisopropylsilyl)but-3-ynoate $(5 \mathrm{~m})$

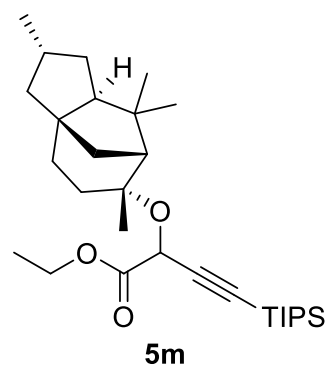

Following general procedure $A$, starting from $(+)$-cedrol $(4 \mathrm{~m})(267 \mathrm{mg}, 1.20 \mathrm{mmol}),((3,3-$ bis(trifluoromethyl)-1 $1 \lambda^{3}$-benzo[d][1,2]iodaoxol-1(3H)-yl)ethynyl)triisopropylsilane (2a) (165 mg, 0.300 $\mathrm{mmol})$, and ethyl 2-diazoacetate (3a) $(1.0 \mathrm{~mL}, 0.60 \mathrm{mmol}, 0.6 \mathrm{M}$ in $\mathrm{DCM})$, afforded the title compound $(5 \mathrm{~m})\left(50: 50 \mathrm{dr}\right.$ in the crude ${ }^{1} \mathrm{H}$ NMR) as a colorless oil $(88 \mathrm{mg}, 0.18 \mathrm{mmol}, 60 \%) . \mathrm{R}_{\mathrm{f}}=0.36$ (EtOAc/pentane 3:97), p-anisaldehyde; ${ }^{1} \mathrm{H}$ NMR $\left(400 \mathrm{MHz}, \mathrm{CDCl}_{3}\right) \delta 4.84$ and $4.82(2 \times \mathrm{s}, 1 \mathrm{H}, \mathrm{HCC} \equiv \mathrm{C})$, $4.31-4.15\left(\mathrm{~m}, 2 \mathrm{H}, \mathrm{OCH}_{2} \mathrm{CH}_{3}\right), 2.12-1.17\left(\mathrm{~m}, 22 \mathrm{H}, \mathrm{CH}\right.$-alphatic and $\left.\mathrm{OCH}_{2} \mathrm{CH}_{3}\right), 1.10-0.93\left(\mathrm{~m}, 24 \mathrm{H}, \mathrm{CH}_{3}\right.$ and TIPS), 0.83 (d, $\left.J=7.1 \mathrm{~Hz}, 3 \mathrm{H}, \mathrm{CHCH}_{3}\right) ;{ }^{13} \mathrm{C} \mathrm{NMR}\left(101 \mathrm{MHz}, \mathrm{CDCl}_{3}\right) \delta 169.5,168.9,103.7,103.1,87.9$, 87.3, 82.3, 82.3, 62.6, 62.2, 61.8, 61.7, 57.7, 57.1, 57.1, 57.0, 54.1, 54.1, 43.5, 43.4, 41.5, 41.4, 41.3, $37.2,37.2,32.9,32.3,31.5,31.4,29.1,29.0,27.7,27.6,25.5,25.3,25.0,18.7,15.8,14.2,11.3$; Not all signals could be resolved. IR $\left(\mathrm{v}_{\max }, \mathrm{cm}^{-1}\right) 2943(\mathrm{~m}), 2866(\mathrm{~m}), 2178(\mathrm{w}), 1765(\mathrm{~s}), 1741(\mathrm{~m}), 1464(\mathrm{~m})$, $1283(\mathrm{~m}), 1269(\mathrm{~m}), 1184(\mathrm{~s}), 1152(\mathrm{~s}), 1091$ (s), 1038 (s), $882(\mathrm{~s}), 756$ (s), 690 (s), 677 (s), 661 (s); HRMS (ESI/QTOF) m/z: [M+Na] ${ }^{+}$Calcd. for $\mathrm{C}_{30} \mathrm{H}_{52} \mathrm{NaO}_{3} \mathrm{Si}^{+}$511.3578; Found 511.3582. 


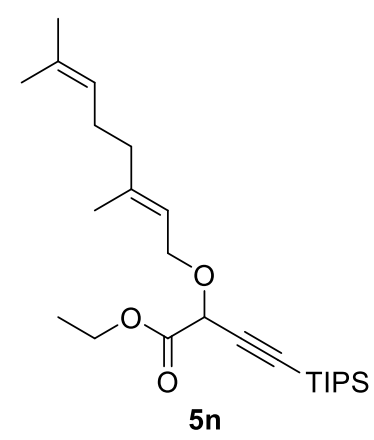

Following general procedure $B$, starting from geraniol $(4 \mathrm{n})(211 \mu \mathrm{L}, 1.20 \mathrm{mmol}), \quad((3,3-$ bis(trifluoromethyl)-1 $1 \lambda^{3}$-benzo[d][1,2]iodaoxol-1(3H)-yl)ethynyl)triisopropylsilane (2a) (165 mg, 0.300 $\mathrm{mmol})$, and ethyl 2-diazoacetate (3a) $(1.0 \mathrm{~mL}, 0.60 \mathrm{mmol}, 0.6 \mathrm{M}$ in DCM), afforded the title compound $(5 \mathrm{n})$ as a colorless oil $(72 \mathrm{mg}, 0.17 \mathrm{mmol}, 57 \%) . \mathrm{R}_{\mathrm{f}}=0.38$ (EtOAc/pentane 3:97), $p$-anisaldehyde; ${ }^{1} \mathrm{H}$ NMR $\left(400 \mathrm{MHz}, \mathrm{CDCl}_{3}\right) \delta 5.35$ (ddt, $J=8.3,7.0,1.3 \mathrm{~Hz}, 1 \mathrm{H}, \mathrm{CHCH}_{2} \mathrm{O}$ ), 5.08 (ddp, $J=7.0,5.8,1.4 \mathrm{~Hz}, 1 \mathrm{H}$, $\left.\left(\mathrm{H}_{3} \mathrm{C}\right)_{2} \mathrm{C}=\mathrm{CH}\right), 4.77$ and $4.68(2 \times \mathrm{s}, 1 \mathrm{H}, \mathrm{HCC} \equiv \mathrm{C}), 4.34-4.18\left(\mathrm{~m}, 4 \mathrm{H}, \mathrm{CHCH}_{2} \mathrm{O}\right.$ and $\left.\mathrm{OCH}_{2} \mathrm{CH}_{3}\right), 2.16-2.01$ $\left(\mathrm{m}, 4 \mathrm{H}, \mathrm{CH}_{2} \mathrm{CH}_{2}\right), 1.69\left(\mathrm{~m}, 6 \mathrm{H}, 2 \times \mathrm{CH}_{3}\right), 1.60\left(\mathrm{~d}, J=1.4 \mathrm{~Hz}, 3 \mathrm{H}, \mathrm{CH}_{3}\right), 1.30\left(\mathrm{t}, J=7.1 \mathrm{~Hz}, 3 \mathrm{H}, \mathrm{OCH}_{2} \mathrm{CH}_{3}\right)$, $1.13-0.94(\mathrm{~m}, 21 \mathrm{H}, \mathrm{TIPS}) ;{ }^{13} \mathrm{C} \mathrm{NMR}\left(101 \mathrm{MHz}, \mathrm{CDCl}_{3}\right) \delta 167.8,142.5,132.0,124.0,119.6,100.6,89.2$, 67.7, 64.9, 62.0, 39.8, 26.4, 25.8, 18.7, 17.8, 16.6, 14.2, 11.3; IR ( $\left.v_{\max }, \mathrm{cm}^{-1}\right) 2959(\mathrm{~s}), 2942(\mathrm{~s}), 2893(\mathrm{~m})$, $2866(\mathrm{~s}), 2174(\mathrm{w}), 1758(\mathrm{~s}), 1463(\mathrm{~m}), 1271(\mathrm{~m}), 1189(\mathrm{~s}), 1103(\mathrm{~s}), 1040(\mathrm{~s}), 883(\mathrm{~s}), 677$ (s), $662(\mathrm{~s})$; HRMS (ESI/QTOF) m/z: [M+Na] $]^{+}$Calcd. for $\mathrm{C}_{25} \mathrm{H}_{44} \mathrm{NaO}_{3} \mathrm{Si}^{+} 443.2952$; Found 443.2953.

Ethyl 2-(((8R,9S,10R,13S,14S,17S)-10,13-dimethyl-3-oxo-2,3,6,7,8,9,10,11,12,13,14,15,16,17tetradecahydro-1H-cyclopenta[a]phenanthren-17-yl)oxy)-4-(triisopropylsilyl)but-3-ynoate (50)<smiles></smiles>

Adapted from general procedure A, starting from testosterone (40) $(260 \mathrm{mg}, 0.900 \mathrm{mmol}, 3$ equiv.), ((3,3-bis(trifluoromethyl)-1 $\lambda^{3}$-benzo[d][1,2]iodaoxol-1(3H)-yl)ethynyl)triisopropylsilane (2a) (165 mg, $0.300 \mathrm{mmol}, 1.00$ equiv.), and ethyl 2-diazoacetate (3a) $(1.00 \mathrm{~mL}, 0.60 \mathrm{mmol}, 0.6 \mathrm{M}$ in DCM, 2.00 equiv.), afforded the title compound (5o) (55:45 dr in the crude ${ }^{1} \mathrm{H} N \mathrm{NMR}$ ) as a thick colorless oil ( $88 \mathrm{mg}$, $0.16 \mathrm{mmol}, 53 \%) \cdot \mathrm{R}_{\mathrm{f}}=0.45$ (EtOAc/pentane 20:80), $p$-anisaldehyde; ${ }^{1} \mathrm{H}$ NMR $\left(400 \mathrm{MHz}, \mathrm{CDCl}_{3}\right) \delta 5.72$ $(\mathrm{s}, 1 \mathrm{H}, H \mathrm{C}=\mathrm{C}), 4.76-4.65(\mathrm{~m}, 1 \mathrm{H}, \mathrm{HCC} \equiv \mathrm{C}), 4.34-4.14\left(\mathrm{~m}, 2 \mathrm{H}, \mathrm{OCH}_{2} \mathrm{CH}_{3}\right), 3.77-3.60(\mathrm{~m}, 1 \mathrm{H}, \mathrm{OCH})$, $2.50-2.16(\mathrm{~m}, 4 \mathrm{H}, \mathrm{CH}$-alky) $), 2.13-1.90(\mathrm{~m}, 3 \mathrm{H}, \mathrm{CH}$-alky) $), 1.88-1.77(\mathrm{~m}, 1 \mathrm{H}, \mathrm{CH}$-alky) $), 1.76-1.50(\mathrm{~m}, 5 \mathrm{H}$, $\mathrm{CH}$-alky), $1.50-0.77$ (m, 36H, $\mathrm{CH}$-alky, $\mathrm{CH}_{3}, \mathrm{CH}_{3}, \mathrm{OCH}_{2} \mathrm{CH}_{3}$ and TIPS); $\left.{ }^{13} \mathrm{C} \mathrm{NMR} \mathrm{(101} \mathrm{MHz,} \mathrm{CDCl}_{3}\right) \delta 199.7$, 199.7, 171.4, 171.3, 168.0, 167.8, 124.0, 101.4, 101.3, 89.0, 88.6, 88.2, 88.2, 87.7, 69.2, 68.9, 61.9, $61.8,54.0,54.0,50.7,50.6,43.1,43.0,38.8,38.8,37.6,37.1,35.9,35.6,34.1,32.9,31.7,31.6,28.2$, $27.7,23.5,20.8,20.7,18.7,17.5,14.2,11.8,11.7,11.3$; Not all signals could be resolved. IR $\left(v_{\max }, \mathrm{cm}^{-1}\right)$ $2919(\mathrm{~s}), 2850(\mathrm{~m}), 1759(\mathrm{~m}), 1672(\mathrm{~m}), 1659(\mathrm{~m}), 1464(\mathrm{~m}), 1268(\mathrm{~m}), 1230(\mathrm{~m}), 1188(\mathrm{~m}), 1158(\mathrm{~m})$, 1115 (s), 1101 (s), 1016 (m), 882 (s), 779 (m), 679 (s); HRMS (ESI/LTQ-Orbitrap) m/z: [M+H] Calcd. for $\mathrm{C}_{34} \mathrm{H}_{55} \mathrm{O}_{4} \mathrm{Si}^{+}$555.3864; Found 555.3859. 
Ethyl 2-(((3aR,5R,5aS,8aS,8bR)-2,2,7,7-tetramethyltetrahydro-5H-bis([1,3]dioxolo)[4,5-b:4',5'd]pyran-5-yl)methoxy)-4-(triisopropylsilyl)but-3-ynoate (5p)

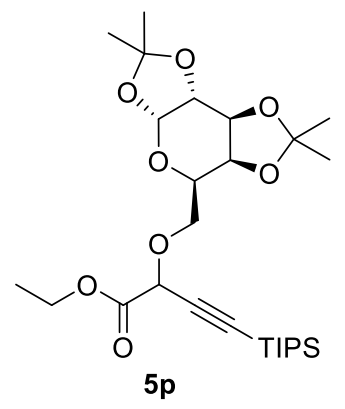

Following general procedure $A$, starting from 1,2:3,4-Di-O-isopropylidene- $\alpha$-D-galactopyranose (5p) (312 $\mathrm{mg}, \quad 1.20 \mathrm{mmol}), \quad\left(\left(3,3-\right.\right.$ bis(trifluoromethyl)-1 $\lambda^{3}$-benzo[d][1,2]iodaoxol-1(3H)yl)ethynyl)triisopropylsilane (2a) $(165 \mathrm{mg}, 0.300 \mathrm{mmol})$, and ethyl 2-diazoacetate (3a) $(1.0 \mathrm{~mL}, 0.60$ mmol, $0.6 \mathrm{M}$ in DCM), afforded the title compound (5p) (58:42 $d r$ in the crude ${ }^{1} \mathrm{H} N M R$ ) as a colorless oil (88 mg, $0.17 \mathrm{mmol}, 56 \%) . \mathrm{R}_{\mathrm{f}}=0.62$ (EtOAc/pentane 20:80), $p$-anisaldehyde; ${ }^{1} \mathrm{H}$ NMR $(400 \mathrm{MHz}$, $\left.\mathrm{CDCl}_{3}\right) \delta 5.52$ (d, $\left.J=5.0 \mathrm{~Hz}, 1 \mathrm{H}, \mathrm{OCH}_{\text {anomer }}\right), 4.94$ and $4.89(2 \mathrm{x} \mathrm{s}, 1 \mathrm{H}, \mathrm{HCC} \equiv \mathrm{C}$ ), 4.60 (ddd, $J=8.0,3.7,2.4$ $\mathrm{Hz}, 1 \mathrm{H}, \mathrm{OCH}), 4.34-4.15\left(\mathrm{~m}, 4 \mathrm{H}, 2 \times \mathrm{OCH}\right.$ and $\left.\mathrm{OCH}_{2} \mathrm{CH}_{3}\right), 4.11-3.96(\mathrm{~m}, 1 \mathrm{H}, \mathrm{OCH}), 3.96-3.79(\mathrm{~m}, 2 \mathrm{H}$, $\left.\mathrm{OCH}_{2}\right), 1.56-1.52\left(\mathrm{~m}, 3 \mathrm{H}, \mathrm{C}\left(\mathrm{CH}_{3}\right)_{2}\right), 1.45-1.41\left(\mathrm{~m}, 3 \mathrm{H}, \mathrm{C}\left(\mathrm{CH}_{3}\right)_{2}\right), 1.35-1.31\left(\mathrm{~m}, 6 \mathrm{H}, \mathrm{C}\left(\mathrm{CH}_{3}\right)_{2}\right), 1.29(\mathrm{t}, \mathrm{J}$ $\left.=7.1 \mathrm{~Hz}, 3 \mathrm{H}, \mathrm{OCH}_{2} \mathrm{CH}_{3}\right), 1.12-0.94(\mathrm{~m}, 21 \mathrm{H}, \mathrm{TIPS}) ;{ }^{13} \mathrm{C} \mathrm{NMR}\left(101 \mathrm{MHz}, \mathrm{CDCl}_{3}\right) \delta 167.8,167.6,109.4$, 109.4, 108.8, 108.7, 100.1, 100.0, 96.5, 96.4, 89.7, 89.7, 71.4, 71.0, 70.8, 70.6, 69.6, 69.3, 67.5, 67.5, $67.0,66.5,62.0,61.9,29.7,26.2,26.1,25.1,24.7,24.6,18.7,14.2,11.2$; Not all signals could be resolved. IR ( $\left.V_{\max } \mathrm{cm}^{-1}\right) 2941(\mathrm{~m}), 2867(\mathrm{w}), 1757(\mathrm{~m}), 1463(\mathrm{w}), 1382(\mathrm{~m}), 1371(\mathrm{~m}), 1255(\mathrm{~m}), 1211$ (s), $1169(\mathrm{~m}), 1109$ (s), $1069(\mathrm{~s}), 1003(\mathrm{~s}), 919(\mathrm{~m}), 884(\mathrm{~m}), 866(\mathrm{~m}), 677(\mathrm{~m})$; HRMS (ESI/QTOF) m/z: $[\mathrm{M}+\mathrm{Na}]^{+}$Calcd. for $\mathrm{C}_{27} \mathrm{H}_{46} \mathrm{NaO}_{8} \mathrm{Si}^{+}$549.2854; Found 549.2855.

Ethyl 2-((2R,3S,5R)-5-(3-benzyl-5-methyl-2,4-dioxo-3,4-dihydropyrimidin-1(2H)-yl)-3(benzyloxy)tetrahydrofuran-2-yl)methoxy)-4-(triisopropylsilyl)but-3-ynoate (5q)

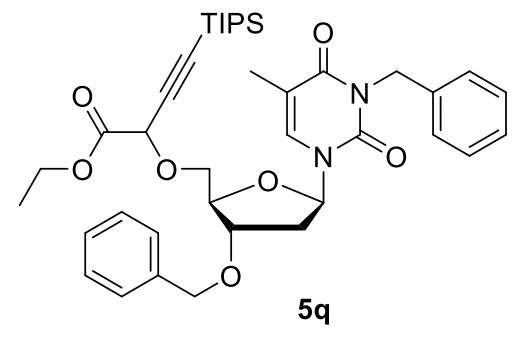

Following general procedure $B$, starting from 3-benzyl-1-((2R,4S,5R)-4-(benzyloxy)-5(hydroxymethyl)tetrahydrofuran-2-yl)-5-methylpyrimidine-2,4(1H,3H)-dione $\quad(\mathbf{4 q}) \quad(507 \quad \mathbf{m g}, \quad 1.20$ mmol), ((3,3-bis(trifluoromethyl)-1 $\lambda^{3}$-benzo[d][1,2]iodaoxol-1(3H)-yl)ethynyl)triisopropylsilane (2a) (165 mg, $0.300 \mathrm{mmol})$, and ethyl 2-diazoacetate (3a) $(1.0 \mathrm{~mL}, 0.60 \mathrm{mmol}, 0.6 \mathrm{M}$ in DCM), afforded the title compound (5q) (53:47 dr in the crude ${ }^{1} \mathrm{H}$ NMR) as a thick colorless oil ( $\left.89 \mathrm{mg}, 0.13 \mathrm{mmol}, 43 \%\right)$. $\mathrm{R}_{\mathrm{f}}$ $=0.38\left(\mathrm{EtOAc} /\right.$ pentane 20:80), $p$-anisaldehyde; ${ }^{1} \mathrm{H}$ NMR $\left(400 \mathrm{MHz}, \mathrm{CDCl}_{3}\right) \delta 7.78$ and $7.73(2 \mathrm{xd}, J=1.4$ $\mathrm{Hz}, 1 \mathrm{H}, H \mathrm{C}=\mathrm{C}), 7.52-7.44(\mathrm{~m}, 2 \mathrm{H}, \mathrm{ArH}), 7.41-7.20(\mathrm{~m}, 8 \mathrm{H}, \mathrm{ArH}), 6.48(\mathrm{td}, J=8.2,5.6 \mathrm{~Hz}, 1 \mathrm{H}, \mathrm{OCHN})$, $5.12\left(\mathrm{~s}, 2 \mathrm{H}, \mathrm{NCH}_{2} \mathrm{Ar}\right), 4.78(\mathrm{~s}, 1 \mathrm{H}, \mathrm{HCC} \equiv \mathrm{C}), 4.57\left(\mathrm{dd}, J=11.7,4.1 \mathrm{~Hz}, 1 \mathrm{H}, \mathrm{OCH}_{2} \mathrm{Ar}\right), 4.49$ (d, J = $11.7 \mathrm{~Hz}$, $\left.1 \mathrm{H}, \mathrm{OCH}_{2} \mathrm{Ar}\right), 4.37-4.16\left(\mathrm{~m}, 4 \mathrm{H}, \mathrm{OCH}_{2} \mathrm{CH}_{3}, \mathrm{BnOCH}\right.$ and $\left.\mathrm{OCH}_{2} \mathrm{CH}\right), 4.09-4.01\left(\mathrm{~m}, 0.5 \mathrm{H}, \mathrm{OCH}_{2} \mathrm{CH}\right), 3.94$ $-3.80\left(\mathrm{~m}, 1 \mathrm{H}, \mathrm{OCH}_{2} \mathrm{CH}\right), 3.70-3.61\left(\mathrm{~m}, 0.5 \mathrm{H}, \mathrm{OCH}_{2} \mathrm{CH}\right), 2.46$ (dddd, $J=13.4,5.5,3.9,1.7 \mathrm{~Hz}, 1 \mathrm{H}, \mathrm{CH}_{2}-$

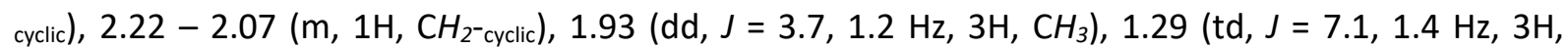
$\left.\mathrm{OCH}_{2} \mathrm{CH}_{3}\right), 1.12-0.91(\mathrm{~m}, 21 \mathrm{H}, \mathrm{TIPS}) ;{ }^{13} \mathrm{C}$ NMR $\left(101 \mathrm{MHz}, \mathrm{CDCl}_{3}\right) \delta 167.0,166.9,163.7,163.7,151.3$, $151.3,137.7,137.7,137.2,137.2,134.6,134.4,129.3,129.3,128.6,128.5,128.0,127.7,127.6,127.6$, 110.6, 110.4, 99.2, 99.0, 91.1, 90.5, 86.0, 85.9, 83.6, 83.6, 80.4, 80.1, 71.4, 71.3, 69.2, 69.1, 68.8, 68.7, 62.2, 44.6, 44.6, 37.6, 37.6, 18.7, 18.6, 14.2, 13.3, 13.2, 11.2; IR ( $\left.\mathrm{V}_{\max } \mathrm{cm}^{-1}\right) 2947(\mathrm{~m}), 2867(\mathrm{~m}), 2175$ 
(w), $1754(\mathrm{~m}), 1701(\mathrm{~s}), 1649(\mathrm{~s}), 1458(\mathrm{~s}), 1273(\mathrm{~m}), 1200(\mathrm{~s}), 1084(\mathrm{~s}), 921(\mathrm{~m}), 885(\mathrm{~m}), 739(\mathrm{~m}), 690$ (s), 672 (s); HRMS (ESI/QTOF) m/z: [M+Na] ${ }^{+}$Calcd. for $\mathrm{C}_{39} \mathrm{H}_{52} \mathrm{~N}_{2} \mathrm{NaO}_{7} \mathrm{Si}+711.3436$; Found 711.3433.

Ethyl 2-((S)-3-(benzyloxy)-2-(((benzyloxy)carbonyl)amino)-3-oxopropoxy)-4-(triisopropylsilyl)but-3ynoate (5r)<smiles>CCOC(=O)C(C#CC(F)(F)C(F)(F)C(F)(F)C(F)(F)F)OCC(NC(=O)OCc1ccccc1)C(=O)OCc1ccccc1</smiles>

Following general procedure $B$, starting from $N$-carbobenzoxy-L-serine benzyl ester $(\mathbf{4 r})(395 \mathrm{mg}, 1.20$ mmol), ((3,3-bis(trifluoromethyl)-1 $\lambda^{3}$-benzo[d] [1,2]iodaoxol-1(3H)-yl)ethynyl)triisopropylsilane (2a) (165 mg, $0.300 \mathrm{mmol})$, and ethyl 2-diazoacetate (3a) $(1.0 \mathrm{~mL}, 0.60 \mathrm{mmol}, 0.6 \mathrm{M}$ in DCM), afforded the title compound ( $5 r)\left(53: 47 d r\right.$ in the crude ${ }^{1} \mathrm{H}$ NMR) as a colorless oil $(65 \mathrm{mg}, 0.11 \mathrm{mmol}, 36 \%)$. $\mathrm{R}_{\mathrm{f}}=0.55$ (EtOAc/pentane 20:80), $p$-anisaldehyde; ${ }^{1} \mathrm{H}$ NMR $\left(400 \mathrm{MHz}, \mathrm{CDCl}_{3}\right) \delta 7.41-7.27(\mathrm{~m}, 10 \mathrm{H}, \mathrm{ArH}), 6.03$ and $5.83(2 \times \mathrm{d}, 8.4 \mathrm{~Hz}, 1 \mathrm{H}, \mathrm{NH}), 5.28-5.05\left(\mathrm{~m}, 4 \mathrm{H}, 2 \times \mathrm{CH}_{2} \mathrm{OAr}\right), 4.85-4.76(\mathrm{~m}, 1 \mathrm{H}, \mathrm{HCC} \equiv \mathrm{C}), 4.63-$ $4.54(\mathrm{~m}, 1 \mathrm{H}, \mathrm{NCH}), 4.33-4.08\left(\mathrm{~m}, 3 \mathrm{H}, \mathrm{OCH}_{2} \mathrm{CH}\right.$ and $\left.\mathrm{OCH}_{2} \mathrm{CH}_{3}\right), 4.00-3.96\left(\mathrm{~m}, 1 \mathrm{H}, \mathrm{OCH}{ }_{2} \mathrm{CH}\right), 1.27(\mathrm{t}, J=$ $\left.7.1 \mathrm{~Hz}, 3 \mathrm{H}, \mathrm{OCH}_{2} \mathrm{CH}_{3}\right), 1.09-0.94(\mathrm{~m}, 21 \mathrm{H}, \mathrm{TIPS}) ;{ }^{13} \mathrm{C} \mathrm{NMR}\left(101 \mathrm{MHz}, \mathrm{CDCl}_{3}\right) \delta 170.0,170.0,167.5$, $167.3,156.3,156.2,136.5,136.4,135.6,135.5,128.7,128.7,128.6,128.6,128.4,128.4,128.2,128.2$, 128.2, 128.2, 128.1, 98.8, 98.6, 91.1, 91.0, 69.0, 68.8, 68.0, 67.5, 67.4, 67.2, 67.1, 62.2, 62.1, 54.5, 54.5, 18.7, 14.2, 11.2; IR ( $\left.v_{\max }, \mathrm{cm}^{-1}\right) 2943(\mathrm{~m}), 2866(\mathrm{~m}), 1746(\mathrm{~s}), 1727(\mathrm{~s}), 1509(\mathrm{~m}), 1457(\mathrm{~m}), 1336(\mathrm{~m})$, $1289(\mathrm{~m}), 1197(\mathrm{~s}), 1120(\mathrm{~s}), 1053(\mathrm{~s}), 882(\mathrm{~m}), 735(\mathrm{~m}), 696$ (s), 677 (s), $664(\mathrm{~m})$; HRMS (ESI/QTOF) m/z: $[\mathrm{M}+\mathrm{Na}]^{+}$Calcd. for $\mathrm{C}_{33} \mathrm{H}_{45} \mathrm{NNaO}_{7} \mathrm{Si}^{+}$618.2858; Found 618.2863.

\section{Ethyl 7-chloro-2-(1-methylcyclopropoxy)hept-3-ynoate (5s)}<smiles>CCOC(=O)C(C#CCCCCl)OC1(C)CC1</smiles>

Following general procedure $A$, starting from 1-methylcyclopropanol $(4 \mathrm{~s})(68.0 \mu \mathrm{L}, 1.20 \mathrm{mmol}), 1-(5-$ chloropent-1-yn-1-yl)-3,3-bis(trifluoromethyl)-1,3-dihydro-1 $\lambda^{3}$-benzo[d]]1,2]iodaoxole (2b) (141 mg, $0.300 \mathrm{mmol}$ ), and ethyl 2-diazoacetate (3a) $(1.0 \mathrm{~mL}, 0.60 \mathrm{mmol}, 0.60 \mathrm{M}$ in DCM), afforded the title compound (5s) as a colorless oil $(27 \mathrm{mg}, 0.10 \mathrm{mmol}, 35 \%) . \mathrm{R}_{\mathrm{f}}=0.11$ (EtOAc/pentane 3:97), $p$ anisaldehyde; ${ }^{1} \mathrm{H}$ NMR $\left(400 \mathrm{MHz}, \mathrm{CDCl}_{3}\right) \delta 4.73$ (t, $\left.J=2.2 \mathrm{~Hz}, 1 \mathrm{H}, \mathrm{HCC} \equiv \mathrm{C}\right), 4.25(\mathrm{q}, J=7.1 \mathrm{~Hz}, 2 \mathrm{H}$, $\mathrm{OCH}_{2} \mathrm{CH}_{3}$ ), $3.63\left(\mathrm{t}, J=6.4 \mathrm{~Hz}, 2 \mathrm{H}, \mathrm{CH}_{2} \mathrm{Cl}\right), 2.42\left(\mathrm{td}, J=6.8,2.2 \mathrm{~Hz}, 2 \mathrm{H}, \mathrm{C} \equiv \mathrm{CCH}_{2}\right), 1.96(\mathrm{p}, J=6.6 \mathrm{~Hz}, 2 \mathrm{H}$, $\left.\mathrm{CH}_{2} \mathrm{CH}_{2} \mathrm{Cl}\right), 1.43\left(\mathrm{~s}, 3 \mathrm{H}, \mathrm{CH}_{3}\right), 1.31\left(\mathrm{t}, \mathrm{J}=7.1 \mathrm{~Hz}, 3 \mathrm{H}, \mathrm{OCH}_{2} \mathrm{CH}_{3}\right), 1.07-1.00(\mathrm{~m}, 1 \mathrm{H}, \mathrm{CH}$-cyclopropyl), $0.93-$ $0.85\left(\mathrm{~m}, 1 \mathrm{H}, \mathrm{CH}\right.$-cyclopropyl), $0.49-0.37\left(\mathrm{~m}, 2 \mathrm{H}, \mathrm{CH}\right.$-cyclopropyl) ${ }^{13} \mathrm{C} \mathrm{NMR}\left(101 \mathrm{MHz}, \mathrm{CDCl}_{3}\right) \delta 168.6,85.8$, 76.4, 67.5, 62.1, 60.2, 43.6, 31.1, 21.2, 16.4, 14.2, 13.9, 13.5; IR $\left(v_{\max }, \mathrm{cm}^{-1}\right) 2963(\mathrm{w}), 2236(\mathrm{w}), 1759$ (s), $1739(\mathrm{~s}), 1445(\mathrm{~m}), 1388(\mathrm{~m}), 1291(\mathrm{~m}), 1251(\mathrm{~s}), 1187(\mathrm{~s}), 1110(\mathrm{~m}), 1075(\mathrm{~s}), 1022(\mathrm{~s}), 857(\mathrm{~m}), 727$ (m), 658 (m); HRMS (ESI/QTOF) m/z: [M+Na] ${ }^{+}$Calcd. for $\mathrm{C}_{13} \mathrm{H}_{19} \mathrm{ClNaO}_{3}{ }^{+}$281.0915; Found 281.0917. 
<smiles>CCCCC#CC(OCCBr)C(=O)OCc1ccccc1</smiles>

Following general procedure $A$, starting from 2-bromoethanol (4t) $(85.0 \mu \mathrm{L}, 1.20 \mathrm{mmol}), 1$-(hex-1-yn1-yl)-3,3-bis(trifluoromethyl)-1,3-dihydro-1 $\lambda^{3}$-benzo[d][1,2]iodaoxole (2c) (135 mg, $0.300 \mathrm{mmol}$ ), and benzyl 2-diazoacetate (3b) $(1.0 \mathrm{~mL}, 0.60 \mathrm{mmol}, 0.6 \mathrm{M}$ in DCM), afforded the title compound (5t) as a colorless oil $(92 \mathrm{mg}, 0.26 \mathrm{mmol}, 87 \%) . \mathrm{R}_{\mathrm{f}}=0.21$ (EtOAc/pentane 3:97), $p$-anisaldehyde; ${ }^{1} \mathrm{H}$ NMR (400 $\left.\mathrm{MHz}, \mathrm{CDCl}_{3}\right) \delta 7.47-7.28(\mathrm{~m}, 5 \mathrm{H}, \mathrm{ArH}), 5.31-5.18\left(\mathrm{~m}, 2 \mathrm{H}, \mathrm{CH}_{2} \mathrm{Ar}\right), 4.85(\mathrm{t}, J=2.2 \mathrm{~Hz}, 1 \mathrm{H}, \mathrm{HCC} \equiv \mathrm{C}), 4.04$ - $3.87\left(\mathrm{~m}, 2 \mathrm{H}, \mathrm{OCH}_{2} \mathrm{CH}_{2} \mathrm{Br}\right), 3.50\left(\mathrm{t}, J=6.5 \mathrm{~Hz}, 2 \mathrm{H}, \mathrm{CH}_{2} \mathrm{Br}\right), 2.24\left(\mathrm{td}, J=7.0,2.2 \mathrm{~Hz}, 2 \mathrm{H}, \mathrm{C} \equiv \mathrm{CCH}_{2}\right), 1.55-$ $1.44\left(\mathrm{~m}, 2 \mathrm{H}, \mathrm{CH}_{2} \mathrm{CH}_{2} \mathrm{CH}_{3}\right), 1.44-1.27\left(\mathrm{~m}, 2 \mathrm{H}, \mathrm{CH}_{2} \mathrm{CH}_{2} \mathrm{CH}_{3}\right), 0.89\left(\mathrm{t}, J=7.3 \mathrm{~Hz}, 3 \mathrm{H}, \mathrm{CH}_{2} \mathrm{CH}_{2} \mathrm{CH}_{3}\right) ;{ }^{13} \mathrm{C}$ NMR $\left(101 \mathrm{MHz}, \mathrm{CDCl}_{3}\right) \delta 167.8,135.3,128.7,128.6,128.3,89.7,72.8,69.1,68.4,67.5,30.4,29.8,22.0,18.6$, 13.7; IR ( $\left.v_{\max }, \mathrm{cm}^{-1}\right) 2959(\mathrm{w}), 2933(\mathrm{w}), 1742(\mathrm{~m}), 1256(\mathrm{~s}), 1213(\mathrm{~s}), 1180(\mathrm{~s}), 1148(\mathrm{~s}), 1110$ (s), 963 (m), $947(\mathrm{~m}), 926(\mathrm{~m}), 756(\mathrm{~s}), 729(\mathrm{~s}), 698(\mathrm{~s}), 680(\mathrm{~m})$; HRMS (ESI/QTOF) m/z: [M+Na] ${ }^{+}$Calcd. for $\mathrm{C}_{17} \mathrm{H}_{21} \mathrm{BrNaO}_{3}{ }^{+}$375.0566; Found 375.0571.

p-Tolyl 4-cyclopropyl-2-(2-(trimethylsilyl)ethoxy)but-3-ynoate (5u)<smiles>CCCOC(C#CC1CC1)C(=O)Oc1ccc(C)cc1</smiles>

Following general procedure A, starting from 2-(trimethylsilyl)ethanol $(4 \mathrm{u})(172 \mu \mathrm{L}, 1.20 \mathrm{mmol}), 1$ (cyclopropylethynyl)-3,3-bis(trifluoromethyl)-1,3-dihydro-1 $\lambda^{3}$-benzo[d][1,2]iodaoxole (2d) (130 mg, $0.300 \mathrm{mmol})$, and $p$-tolyl 2-diazoacetate $(3 \mathrm{c})(1.0 \mathrm{~mL}, 0.60 \mathrm{mmol}, 0.60 \mathrm{M}$ in DCM), afforded the title compound (5u) as a colorless oil $(67 \mathrm{mg}, 0.20 \mathrm{mmol}, 68 \%) . \mathrm{R}_{\mathrm{f}}=0.34$ (EtOAc/pentane 3:97), $p$ anisaldehyde; ${ }^{1} \mathrm{H}$ NMR $\left(400 \mathrm{MHz}, \mathrm{CDCl}_{3}\right) \delta 7.21-7.13(\mathrm{~m}, 2 \mathrm{H}, \mathrm{ArH}), 7.05-6.98(\mathrm{~m}, 2 \mathrm{H}, \mathrm{ArH}), 4.85(\mathrm{~d}, J$ $=2.0 \mathrm{~Hz}, 1 \mathrm{H}, \mathrm{HCC} \equiv \mathrm{C}), 3.84-3.75\left(\mathrm{~m}, 1 \mathrm{H}, \mathrm{OCH}_{2}\right), 3.75-3.66\left(\mathrm{~m}, 1 \mathrm{H}, \mathrm{OCH}_{2}\right), 2.34\left(\mathrm{~s}, 3 \mathrm{H}, \mathrm{ArCH}_{3}\right), 1.37-$ $1.27\left(\mathrm{~m}, 1 \mathrm{H}, \mathrm{CH}\right.$-cyclopropyl), $1.09-1.00\left(\mathrm{~m}, 2 \mathrm{H}, \mathrm{CH}_{2} \mathrm{TMS}\right), 0.86-0.72(\mathrm{~m}, 4 \mathrm{H}, \mathrm{CH}$-cyclopropyl), $0.04(\mathrm{~s}, 9 \mathrm{H}$, TMS); ${ }^{13} \mathrm{C} \mathrm{NMR}\left(101 \mathrm{MHz}, \mathrm{CDCl}_{3}\right) \delta 167.0,148.4,135.9,130.1,121.0,91.8,68.8,68.6,66.8,21.0,18.2$, 8.5, 8.5, -0.3, -1.3; IR ( $\left.v_{\max }, \mathrm{cm}^{-1}\right) 2953(\mathrm{w}), 2895(\mathrm{w}), 2238(\mathrm{w}), 1775(\mathrm{~m}), 1507(\mathrm{~m}), 1248(\mathrm{~m}), 1195(\mathrm{~s})$, 1166 (s), 1095 (s), 857 (s), 835 (s), 695 (m); HRMS (ESI/QTOF) m/z: [M+Na] ${ }^{+}$Calcd. for $\mathrm{C}_{19} \mathrm{H}_{26} \mathrm{NaO}_{3} \mathrm{Si}^{+}$ 353.1543; Found 353.1543.

2,6-Di-tert-butyl-4-methylphenyl 2-((3s,5s,7s)-adamantan-1-yloxy)-4-(triisopropylsilyl)but-3-ynoate (5v)<smiles>Cc1cc(Br)c(OC(=O)C(C#CC(F)(F)F)OC2C=CC3CCC2C3)c(Br)c1</smiles>

Following general procedure A, starting from 1-adamantanol (4v) $(183 \mathrm{mg}, 1.20 \mathrm{mmol}),((3,3-$ bis(trifluoromethyl)-1 $1 \lambda^{3}$-benzo[d][1,2]iodaoxol-1(3H)-yl)ethynyl)triisopropylsilane (2a) (165 mg, 0.300 $\mathrm{mmol}$ ), and 1,3-di-tert-butyl-2-(diazomethyl)-5-methylbenzene (3d) $(1.0 \mathrm{~mL}, 0.60 \mathrm{mmol}, 0.60 \mathrm{M}$ in $D C M)$, afforded the title compound ( $5 \mathrm{v}$ ) as a viscous colorless oil (132 $\mathrm{mg}, 0.220 \mathrm{mmol}, 74 \%) . \mathrm{R}_{\mathrm{f}}=0.43$ (EtOAc/pentane 3:97), $p$-anisaldehyde; ${ }^{1} \mathrm{H}$ NMR (400 MHz, $\left.\mathrm{CDCl}_{3}\right) \delta 7.11(\mathrm{~s}, 2 \mathrm{H}, \mathrm{ArH}), 5.18(\mathrm{~s}, 1 \mathrm{H}$, 
$H C C \equiv C), 2.31\left(\mathrm{~s}, 3 \mathrm{H}, \mathrm{CH}_{3}\right), 2.24-2.15(\mathrm{~m}, 3 \mathrm{H}, 3 \times \mathrm{CH}), 2.01-1.87\left(\mathrm{~m}, 6 \mathrm{H}, \mathrm{C}\left(\mathrm{CH}_{2}\right)_{3}\right), 1.73-1.59(\mathrm{~m}, 6 \mathrm{H}$, $\left.3 \times \mathrm{CH}_{2}\right), 1.35(\mathrm{~m}, 18 \mathrm{H}, 2 \times t B u), 1.08(\mathrm{~s}, 21 \mathrm{H}, \mathrm{TIPS}) ;{ }^{13} \mathrm{C} \mathrm{NMR}\left(101 \mathrm{MHz}, \mathrm{CDCl}_{3}\right) \delta 168.1,146.4,142.3$, 142.1, 134.8, 127.1, 127.1, 103.8, 89.0, 76.2, 62.5, 42.3, 36.4, 35.5, 35.5, 31.8, 31.7, 30.9, 21.7, 18.7, 18.7, 11.4; IR ( $\left.v_{\max }, \mathrm{cm}^{-1}\right) 2912(\mathrm{~m}), 2865(\mathrm{~m}), 2251(\mathrm{w}), 2176(\mathrm{w}), 1760(\mathrm{~m}), 1462(\mathrm{~m}), 1421(\mathrm{~m}), 1364$ (m), $1271(\mathrm{~m}), 1200(\mathrm{~m}), 1183(\mathrm{~m}), 1144(\mathrm{~m}), 1104(\mathrm{~s}), 1074(\mathrm{~s}), 1018(\mathrm{~m}), 909(\mathrm{~s}), 883(\mathrm{~m}), 733(\mathrm{~s}), 677$ (s); HRMS (ESI/QTOF) m/z: [M+K] ${ }^{+}$Calcd. for $\mathrm{C}_{38} \mathrm{H}_{60} \mathrm{KO}_{3} \mathrm{Si}^{+}$631.3943; Found 631.3958.

Furan-2-ylmethyl 4-(cyclohex-1-en-1-yl)-2-((3-(trimethylsilyl)prop-2-yn-1-yl)oxy)but-3-ynoate (5w)<smiles>CC#CCOC(C#CC1=CCCCC1)C(=O)OCc1ccco1</smiles>

Following general procedure B, starting from 3-trimethylsilyl-2-propyn-1-ol (4w) (148 $\mu \mathrm{L}, 1.20 \mathrm{mmol})$, 1-(cyclohex-1-en-1-ylethynyl)-3,3-bis(trifluoromethyl)-1,3-dihydro-1 $\lambda^{3}$-benzo[d][1,2]iodaoxole (2e) (119 $\mathrm{mg}, 0.300 \mathrm{mmol})$, and furan-2-ylmethyl 2-diazoacetate (3e) $(1.0 \mathrm{~mL}, 0.60 \mathrm{mmol}, 0.60 \mathrm{M}$ in DCM), afforded the title compound $(\mathbf{5 w})$ as a viscous colorless oil ( $36 \mathrm{mg}, 0.10 \mathrm{mmol}, 39 \%$ ). $\mathrm{R}_{\mathrm{f}}=0.21$ (EtOAc/pentane 3:97), $p$-anisaldehyde; ${ }^{1} \mathrm{H} \mathrm{NMR}\left(400 \mathrm{MHz}, \mathrm{CDCl}_{3}\right) \delta 7.42$ (dd, $\left.J=1.9,0.9 \mathrm{~Hz}, 1 \mathrm{H}, \mathrm{ArH}\right)$, $6.49-6.42(\mathrm{~m}, 1 \mathrm{H}, \mathrm{ArH}), 6.36(\mathrm{dd}, J=3.3,1.9 \mathrm{~Hz}, 1 \mathrm{H}, \mathrm{ArH}), 6.16(\mathrm{p}, J=2.1 \mathrm{~Hz}, 1 \mathrm{H}, \mathrm{C}=\mathrm{CH}), 5.25-5.13$ $\left(\mathrm{m}, 2 \mathrm{H}, \mathrm{OCH}_{2} \mathrm{Ar}\right), 5.08(\mathrm{~s}, 1 \mathrm{H}, \mathrm{HCC} \equiv \mathrm{C}), 4.37\left(\mathrm{~s}, 2 \mathrm{H}, \mathrm{OCH}_{2} \mathrm{C} \equiv \mathrm{C}\right), 2.14-2.04(\mathrm{~m}, 4 \mathrm{H}, \mathrm{CH}$-cyclohexenyl), $1.68-$ $1.51(\mathrm{~m}, 4 \mathrm{H}, \mathrm{CH}$-cyclohexeny) $), 0.16$ (s, 9H, TMS); $\left.{ }^{13} \mathrm{C} \mathrm{NMR} \mathrm{(101} \mathrm{MHz,} \mathrm{CDCl} 3\right) \delta 167.2,148.8,143.6,137.6$, 119.7, 111.4, 110.8, 100.0, 92.9, 90.0, 78.7, 67.5, 59.5, 56.7, 28.9, 25.8, 22.3, 21.5, -0.1; IR $\left(v_{\max } \mathrm{cm}^{-1}\right)$ $2934(\mathrm{~m}), 2862(\mathrm{w}), 1753(\mathrm{~m}), 1444(\mathrm{w}), 1251(\mathrm{~m}), 1181(\mathrm{~m}), 1090(\mathrm{~s}), 1009(\mathrm{~m}), 846(\mathrm{~s}), 753(\mathrm{~m})$; HRMS (ESI/QTOF) m/z: [M+Na] $]^{+}$Calcd. for $\mathrm{C}_{21} \mathrm{H}_{26} \mathrm{NaO}_{4} \mathrm{Si}^{+}$393.1493; Found 393.1491.

\section{2-Butoxy-4-(triisopropylsilyl)but-3-ynenitrile (5x)}

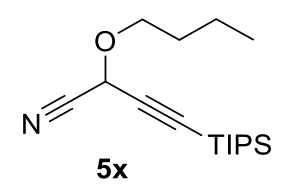

Following general procedure A, starting from 1-butanol $(\mathbf{4 x})(110 \mu \mathrm{L}, 1.20 \mathrm{mmol}), \quad((3,3-$ bis(trifluoromethyl)-1 $\lambda^{3}$-benzo[d][1,2]iodaoxol-1(3H)-yl)ethynyl)triisopropylsilane (2a) (165 mg, 0.300 $\mathrm{mmol})$, and 2-diazoacetonitrile (3f) $(1.20 \mathrm{~mL}, 0.600 \mathrm{mmol}, 0.50 \mathrm{M}$ in DCM), afforded the title compound (5x) as a colorless oil $(57 \mathrm{mg}, 0.19 \mathrm{mmol}, 65 \%) . \mathrm{R}_{\mathrm{f}}=0.14$ (EtOAc/pentane 3:97), $p$ anisaldehyde; ${ }^{1} \mathrm{H}$ NMR (400 MHz, $\left.\mathrm{CDCl}_{3}\right) \delta 5.17(\mathrm{~s}, 1 \mathrm{H}, \mathrm{HCC} \equiv \mathrm{C}), 3.82-3.72\left(\mathrm{~m}, 1 \mathrm{H}, \mathrm{OCH}_{2}\right), 3.69-3.61$ $\left(\mathrm{m}, 1 \mathrm{H}, \mathrm{OCH}_{2}\right), 1.63\left(\mathrm{tt}, \mathrm{J}=8.3,6.3 \mathrm{~Hz}, 2 \mathrm{H}, \mathrm{OCH}_{2} \mathrm{CH}_{2}\right), 1.47-1.35\left(\mathrm{~m}, 2 \mathrm{H}, \mathrm{CH}_{2} \mathrm{CH}_{3}\right), 1.14-0.97(\mathrm{~m}, 21 \mathrm{H}$, TIPS), $0.93\left(\mathrm{t}, J=7.4 \mathrm{~Hz}, 3 \mathrm{H}, \mathrm{CH}_{2} \mathrm{CH}_{3}\right) ;{ }^{13} \mathrm{C} \mathrm{NMR}\left(101 \mathrm{MHz}, \mathrm{CDCl}_{3}\right) \delta 114.9,96.3,91.8,68.3,58.0,31.3$, 19.3, 18.6, 13.9, 11.1; IR ( $\left.v_{\max }, \mathrm{cm}^{-1}\right) 2945(\mathrm{~m}), 2867(\mathrm{~m}), 1740(\mathrm{~m}), 1717(\mathrm{~m}), 1464(\mathrm{~m}), 1253(\mathrm{~m}), 1091$ (s), $1029(\mathrm{~m}), 882(\mathrm{~s}), 836(\mathrm{~m}), 776(\mathrm{~m}), 678(\mathrm{~s}), 662(\mathrm{~s})$; HRMS (APCI/QTOF) m/z: [M+H] ${ }^{+}$Calcd. for $\mathrm{C}_{17} \mathrm{H}_{32} \mathrm{NOSi}^{+}$294.2248; Found 294.2251. 
<smiles>CCOP(=O)(OCC)C(C#Cc1ccccc1Br)OCCC(C)=O</smiles>

Following general procedure $A$, starting from 4-hydroxy-2-butanone (4y) (103 $\mu \mathrm{L}, 1.20 \mathrm{mmol}), 1-((2-$ bromophenyl)ethynyl)-3,3-bis(trifluoromethyl)-1,3-dihydro-1 $\lambda^{3}$-benzo[d][1,2]iodaoxole (2f) (165 mg, $0.300 \mathrm{mmol}$ ) and diethyl (diazomethyl)phosphonate $(3 \mathrm{~g})(1.00 \mathrm{~mL}, 0.600 \mathrm{mmol}, 0.60 \mathrm{M}$ in DCM), afforded the title compound (5y) as a colorless oil $(95 \mathrm{mg}, 0.23 \mathrm{mmol}, 76 \%) . \mathrm{R}_{\mathrm{f}}=0.17$ (EtOAc/pentane 20:80), $p$-anisaldehyde; ${ }^{1} \mathrm{H}$ NMR $\left(400 \mathrm{MHz}, \mathrm{CDCl}_{3}\right) \delta 7.58$ (dd, $\left.J=8.0,1.3 \mathrm{~Hz}, 1 \mathrm{H}, \operatorname{ArH}\right), 7.50$ (dd, $J=7.6$, $1.7 \mathrm{~Hz}, 1 \mathrm{H}, \mathrm{ArH}), 7.30-7.23(\mathrm{~m}, 1 \mathrm{H}, \mathrm{ArH}), 7.20(\mathrm{td}, J=7.7,1.8 \mathrm{~Hz}, 1 \mathrm{H}, \operatorname{ArH}), 4.73(\mathrm{~d}, J=19.4 \mathrm{~Hz}, 1 \mathrm{H}$, $\mathrm{HCC} \equiv \mathrm{C}), 4.34-4.18\left(\mathrm{~m}, 5 \mathrm{H}, 2 \times \mathrm{POCH}_{2} \mathrm{CH}_{3}\right.$ and $\left.\mathrm{OCH}_{2}\right), 3.92$ (ddd, $\left.J=9.6,6.8,5.9 \mathrm{~Hz}, 1 \mathrm{H}, \mathrm{OCH}_{2}\right), 2.88-$ $2.70\left(\mathrm{~m}, 2 \mathrm{H}, \mathrm{CH}_{2} \mathrm{COCH}_{3}\right), 2.20\left(\mathrm{~s}, 3 \mathrm{H}, \mathrm{COCH}_{3}\right), 1.36\left(\mathrm{tt}, J=7.0,1.0 \mathrm{~Hz}, 6 \mathrm{H}, 2 \times \mathrm{POCH}_{2} \mathrm{CH}_{3}\right) ;{ }^{13} \mathrm{C}_{\mathrm{NMR}}(101$ $\left.\mathrm{MHz}, \mathrm{CDCl}_{3}\right) \delta 206.7,133.9(\mathrm{~d}, J=2.8 \mathrm{~Hz}), 132.6,130.2,127.2,125.7(\mathrm{~d}, J=3.1 \mathrm{~Hz}), 124.3(\mathrm{~d}, J=3.6 \mathrm{~Hz})$, $87.7(\mathrm{~d}, J=10.0 \mathrm{~Hz}), 86.1(\mathrm{~d}, J=5.2 \mathrm{~Hz}), 67.1(\mathrm{~d}, J=175.3 \mathrm{~Hz}), 66.0(\mathrm{~d}, J=12.5 \mathrm{~Hz}), 64.1(\mathrm{dd}, J=11.1$, 6.7 Hz), 43.6, 30.5, $16.7(\mathrm{dd}, J=5.7,3.8 \mathrm{~Hz}) ;{ }^{31} \mathrm{P} \mathrm{NMR}\left(162 \mathrm{MHz}, \mathrm{CDCl}_{3}\right) \delta 14.3 ; \mathrm{IR}\left(\mathrm{v}_{\max }, \mathrm{cm}^{-1}\right) 2917(\mathrm{w})$, $2849(\mathrm{w}), 1715(\mathrm{~m}), 1469(\mathrm{w}), 1236(\mathrm{~m}), 1165(\mathrm{w}), 1097(\mathrm{~m}), 1021(\mathrm{~s}), 977(\mathrm{~m}), 756(\mathrm{~m})$; HRMS (APPI/LTQ-Orbitrap) m/z: [M+H] ${ }^{+}$Calcd. for $\mathrm{C}_{17} \mathrm{H}_{23} \mathrm{BrO}_{5} \mathrm{P}^{+}$417.0461; Found 417.0455.

\section{Ethyl 7-((tert-butyldiphenylsilyl)oxy)-1-(cyclobutylmethoxy)hept-2-yne-1-sulfonate (5z)}

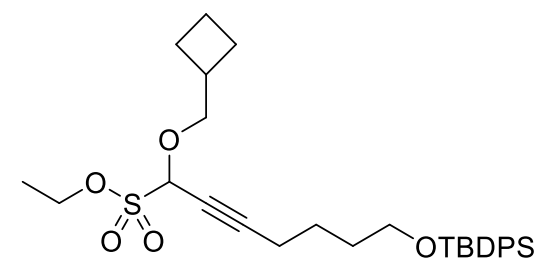

$5 z$

Following general procedure $A$, starting from cyclobutanemethanol (4z) $(113 \mu \mathrm{L}, 1.20 \mathrm{mmol}),((6-(3,3-$ bis(trifluoromethyl)-1 $\lambda^{3}$-benzo[d][1,2]iodaoxol-1(3H)-yl)hex-5-yn-1-yl)oxy)(tert-butyl)diphenylsilane $(2 \mathrm{~g})(211 \mathrm{mg}, 0.300 \mathrm{mmol})$, and ethyl diazomethanesulfonate $(3 \mathrm{~h})(1.0 \mathrm{~mL}, 0.60 \mathrm{mmol}, 0.60 \mathrm{M}$ in DCM), afforded the title compound $(\mathbf{5 z})$ as a colorless oil $(132 \mathrm{mg}, 0.240 \mathrm{mmol}, 81 \%) . \mathrm{R}_{\mathrm{f}}=0.35$ (EtOAc/pentane 3:97), $p$-anisaldehyde; ${ }^{1} \mathrm{H}$ NMR $\left(400 \mathrm{MHz}, \mathrm{CDCl}_{3}\right) \delta 7.70-7.62(\mathrm{~m}, 4 \mathrm{H}, \mathrm{ArH}), 7.49-$ $7.33(\mathrm{~m}, 6 \mathrm{H}, \mathrm{ArH}), 5.25(\mathrm{t}, J=1.6 \mathrm{~Hz}, 1 \mathrm{H}, H C \mathrm{CC}=\mathrm{C}), 3.80-3.45\left(\mathrm{~m}, 6 \mathrm{H}, 3 \times \mathrm{OCH}_{2}\right), 2.58$ (hept, $J=7.4 \mathrm{~Hz}$, $\left.1 \mathrm{H}, \mathrm{OCH}_{2} \mathrm{CH}\right), 2.31-2.21\left(\mathrm{~m}, 2 \mathrm{H}, \mathrm{CH}_{2}\right.$-aliphatic $), 2.13-2.00\left(\mathrm{~m}, 2 \mathrm{H}, \mathrm{CH}_{2^{-} \text {aliphatic }}\right), 1.98-1.80\left(\mathrm{~m}, 2 \mathrm{H}, \mathrm{CH}_{2^{-}}\right.$ aliphatic), $1.80-1.59\left(\mathrm{~m}, 6 \mathrm{H}, \mathrm{CH}_{2}\right.$-aliphatic), $1.23\left(\mathrm{t}, \mathrm{J}=7.1 \mathrm{~Hz}, 3 \mathrm{H}, \mathrm{OCH}_{2} \mathrm{CH}_{3}\right), 1.05(\mathrm{~s}, 9 \mathrm{H}, \mathrm{tBu}) ;{ }^{13} \mathrm{C} \mathrm{NMR}(101$ $\left.\mathrm{MHz}, \mathrm{CDCl}_{3}\right) \delta 135.7,134.1,129.7,127.8,91.7,86.5,76.0,69.4,63.4,61.0,35.0,31.9,27.0,25.3,25.2$, 25.0, 19.4, 18.7, 18.6, 15.2; IR $\left(v_{\max }, \mathrm{cm}^{-1}\right) 2931(\mathrm{~m}), 2859(\mathrm{~m}), 2245(\mathrm{w}), 1428(\mathrm{~m}), 1389(\mathrm{w}), 1359(\mathrm{~m})$, $1148(\mathrm{~m}), 1108(\mathrm{~s}), 1041(\mathrm{~s}), 1008(\mathrm{~m}), 740(\mathrm{~m}), 701$ (s), $613(\mathrm{~m})$; HRMS (APPI/LTQO) m/z: [M- $\left.{ }_{2} \mathrm{H}_{5} \mathrm{O}_{3} \mathrm{~S}\right]^{+}$ Calcd. for $\mathrm{C}_{28} \mathrm{H}_{37} \mathrm{O}_{2} \mathrm{Si}^{+}$433.2557; Found 433.2543 . 


\section{(3-(2-(Benzyloxy)ethoxy)-4,4,4-trifluorobut-1-yn-1-yl)benzene (5aa)}<smiles>FC(F)(F)C(C#Cc1ccccc1)OCCOCc1ccccc1</smiles>

Following general procedure A, starting from 2-(benzyloxy)ethanol (4aa) (171 $\mu \mathrm{L}, 1.20 \mathrm{mmol}), 1$ (phenylethynyl)-3,3-bis(trifluoromethyl)-1,3-dihydro-1 $\lambda^{3}$-benzo[d][1,2]iodaoxole (2h) (141 mg, 0.300 $\mathrm{mmol})$, and 2,2,2-trifluorodiazoethane (3i) $(1.62 \mathrm{~mL}, 0.600 \mathrm{mmol}, 0.37 \mathrm{M}$ in DCM), afforded the title compound (5aa) as a colorless oil $(73 \mathrm{mg}, 0.22 \mathrm{mmol}, 73 \%) . \mathrm{R}_{\mathrm{f}}=0.27$ (EtOAc/pentane 3:97), $p$ anisaldehyde; ${ }^{1} \mathrm{H}$ NMR $\left(400 \mathrm{MHz}, \mathrm{CDCl}_{3}\right) \delta 7.52-7.46(\mathrm{~m}, 2 \mathrm{H}, \mathrm{ArH}), 7.41-7.26(\mathrm{~m}, 8 \mathrm{H}, \mathrm{ArH}), 4.90(\mathrm{q}, J$ $=5.9 \mathrm{~Hz}, 1 \mathrm{H}, \mathrm{HCC} \equiv \mathrm{C}), 4.60\left(\mathrm{~s}, 2 \mathrm{H}, \mathrm{CH}_{2} \mathrm{Ar}\right), 4.06-3.98\left(\mathrm{~m}, 1 \mathrm{H}, \mathrm{OCH}_{2}\right), 3.98-3.90\left(\mathrm{~m}, 1 \mathrm{H}, \mathrm{OCH}_{2}\right), 3.80-$ $3.66\left(\mathrm{~m}, 2 \mathrm{H}, \mathrm{CH}_{2} \mathrm{OBn}\right) ;{ }^{13} \mathrm{C} \mathrm{NMR}\left(101 \mathrm{MHz}, \mathrm{CDCl}_{3}\right) \delta 138.2,132.2,129.5,128.6,128.5,127.9,127.8$, $122.7(\mathrm{q}, J=281.8 \mathrm{~Hz}), 121.3,88.8,79.3(\mathrm{q}, J=2.4 \mathrm{~Hz}), 73.5,69.8(\mathrm{q}, J=35.1 \mathrm{~Hz}), 69.4,69.4 ;{ }^{19} \mathrm{~F} \mathrm{NMR}$ $\left(376 \mathrm{MHz}, \mathrm{CDCl}_{3}\right) \delta$-76.8; IR $\left(\mathrm{v}_{\max }, \mathrm{cm}^{-1}\right) 2871(\mathrm{w}), 1720(\mathrm{w}), 1703(\mathrm{w}), 1491(\mathrm{w}), 1454(\mathrm{w}), 1362(\mathrm{w})$, 1272 (s), 1184 (s), 1141 (s), 1095 (s), 1028 (m), 756 (s), 690 (s); HRMS (APPI/LTQ-Orbitrap) m/z: [M] ${ }^{+}$ Calcd. for $\mathrm{C}_{19} \mathrm{H}_{17} \mathrm{~F}_{3} \mathrm{O}_{2}{ }^{+}$334.1175; Found 334.1173.

\section{Benzyl 3-((1,1,1-trifluoro-4-(triisopropylsilyl)but-3-yn-2-yl)oxy)azetidine-1-carboxylate (5ab)}<smiles>O=C(OCc1ccccc1)N1CC(OC(C#C[PbH2])C(F)(F)F)C1</smiles>

Following general procedure B, starting from benzyl 3-hydroxyazetidine-1-carboxylate (4ab) (249 mg, $1.20 \mathrm{mmol}),\left(\left(3,3\right.\right.$-bis(trifluoromethyl)-1 $\lambda^{3}$-benzo[d][1,2]iodaoxol-1(3H)-yl)ethynyl)triisopropylsilane (2a) (165 mg, $0.300 \mathrm{mmol})$, and 2,2,2-trifluorodiazoethane (3i) (1.67 mL, $0.600 \mathrm{mmol}, 0.36 \mathrm{M}$ in DCM), afforded the title compound ( $5 \mathrm{ab}$ ) as a colorless oil $(86 \mathrm{mg}, 0.18 \mathrm{mmol}, 61 \%) . \mathrm{R}_{\mathrm{f}}=0.09$ (EtOAc/pentane 3:97), $p$-anisaldehyde; ${ }^{1} \mathrm{H}$ NMR ( $\left.400 \mathrm{MHz}, \mathrm{CDCl}_{3}\right) \delta 7.40-7.28(\mathrm{~m}, 5 \mathrm{H}, \mathrm{ArH}), 5.10\left(\mathrm{~s}, 2 \mathrm{H}, \mathrm{OCH}_{2} \mathrm{Ar}\right), 4.73$ $-4.63(\mathrm{~m}, 1 \mathrm{H}, \mathrm{OCH}), 4.55(\mathrm{q}, J=5.8 \mathrm{~Hz}, 1 \mathrm{H}, \mathrm{HCC} \equiv \mathrm{C}), 4.30-4.16\left(\mathrm{~m}, 2 \mathrm{H}, \mathrm{NCH}_{2}\right), 4.11$ (ddd, $J=9.7,4.4$, $\left.1.1 \mathrm{~Hz}, 1 \mathrm{H}, \mathrm{NCH}_{2}\right), 4.01$ (ddd, $\left.J=9.8,4.4,1.2 \mathrm{~Hz}, 1 \mathrm{H}, \mathrm{NCH}_{2}\right), 1.14-0.95(\mathrm{~m}, 21 \mathrm{H}, \mathrm{TIPS}) ;{ }^{13} \mathrm{C}$ NMR $(101$ $\left.\mathrm{MHz}, \mathrm{CDCl}_{3}\right) \delta 156.4,136.6,128.6,128.2,128.1,122.1(\mathrm{q}, J=281.9 \mathrm{~Hz}), 95.7,93.5,68.2(\mathrm{q}, J=35.5 \mathrm{~Hz})$, 67.7, 67.0, 57.9-56.3 (m), 18.6, 11.1; $\left.{ }^{19} \mathrm{~F} \mathrm{NMR} \mathrm{(376} \mathrm{MHz,} \mathrm{CDCl} 3\right) \delta-77.0 ; \mathrm{IR}\left(\mathrm{v}_{\max }, \mathrm{cm}^{-1}\right) 2946(\mathrm{~m}), 2867$ $(\mathrm{m}), 1713(\mathrm{~s}), 1456(\mathrm{~m}), 1418(\mathrm{~s}), 1352(\mathrm{~m}), 1272(\mathrm{~m}), 1181(\mathrm{~s}), 1146(\mathrm{~s}), 1093(\mathrm{~s}), 1039(\mathrm{~m}), 1001(\mathrm{~m})$, $882(\mathrm{~m}), 736(\mathrm{~m}), 697(\mathrm{~m}), 680(\mathrm{~s})$; HRMS (ESI/QTOF) m/z: [M+Na] ${ }^{+}$Calcd. for $\mathrm{C}_{24} \mathrm{H}_{34} \mathrm{~F}_{3} \mathrm{NNaO}_{3} \mathrm{Si}_{+}$ 492.2152; Found 492.2164 . 
Triisopropyl(4,4,5,5,5-pentafluoro-3-((3-(4,4,5,5-tetramethyl-1,3,2-dioxaborolan-2yl)benzyl)oxy)pent-1-yn-1-yl)silane (5ac)

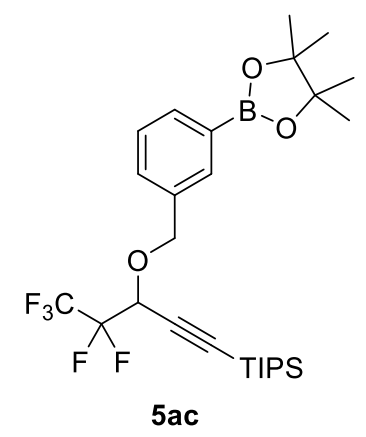

Following general procedure A, starting from (3-(4,4,5,5-tetramethyl-1,3,2-dioxaborolan-2yl)phenyl)methanol (4ac) $(281 \mathrm{mg}, 1.20 \mathrm{mmol}),\left(\left(3,3-b i s(t r i f l u o r o m e t h y l)-1 \lambda^{3}\right.\right.$-benzo[d][1,2]iodaoxol1(3H)-yl)ethynyl)triisopropylsilane (2i) (165 $\mathrm{mg}, 0.300 \mathrm{mmol})$, and 3-diazo-1,1,1,2,2pentafluoropropane (3j) $(1.67 \mathrm{~mL}, 0.600 \mathrm{mmol}, 0.36 \mathrm{M}$ in DCM), afforded the title compound (5ac) as a colorless oil $(128 \mathrm{mg}, 0.234 \mathrm{mmol}, 78 \%) . \mathrm{R}_{\mathrm{f}}=0.40$ (EtOAc/pentane 3:97), $p$-anisaldehyde; ${ }^{1} \mathrm{H} \mathrm{NMR}$ $\left(400 \mathrm{MHz}, \mathrm{CDCl}_{3}\right) \delta 7.83-7.73(\mathrm{~m}, 2 \mathrm{H}, \mathrm{ArH}), 7.49(\mathrm{dt}, J=7.7,1.7 \mathrm{~Hz}, 1 \mathrm{H}, \mathrm{ArH}), 7.44-7.34(\mathrm{~m}, 1 \mathrm{H}, \mathrm{ArH})$, $4.88\left(\mathrm{~d}, J=11.7 \mathrm{~Hz}, 1 \mathrm{H}, \mathrm{CH}_{2} \mathrm{O}\right), 4.77-4.66\left(\mathrm{~m}, 1 \mathrm{H}, \mathrm{CH}_{2} \mathrm{O}\right), 4.66-4.54(\mathrm{~m}, 1 \mathrm{H}, \mathrm{HCC} \equiv \mathrm{C}), 1.35(\mathrm{~s}, 12 \mathrm{H}, 4 \mathrm{x}$ $\left.\mathrm{CH}_{3}\right), 1.19-0.96(\mathrm{~m}, 21 \mathrm{H}, \mathrm{TIPS}) ;{ }^{13} \mathrm{C}$ NMR $\left(101 \mathrm{MHz}, \mathrm{CDCl}_{3}\right) \delta 135.3,134.9,134.9,131.4,129.4$ (br s), $128.2,118.8$ (tq, $J=287.0,35.0 \mathrm{~Hz}$ ), 111.8 (qdd, $J=256.8,36.3,5.0 \mathrm{~Hz}), 95.9,93.3,84.0,71.0,67.3$ (dd, $J=29.5,24.8 \mathrm{~Hz}), 25.01(\mathrm{~d}, J=4.7 \mathrm{~Hz}), 18.6,11.2 ;{ }^{19} \mathrm{~F} \mathrm{NMR}\left(376 \mathrm{MHz}, \mathrm{CDCl}_{3}\right) \delta-81.1,-119.9$ (d, $J=273.9$ $\mathrm{Hz}),-125.3(\mathrm{~d}, J=274.2 \mathrm{~Hz}) ; \mathrm{IR}\left(\mathrm{v}_{\max }, \mathrm{cm}^{-1}\right) 2945(\mathrm{~m}), 2868(\mathrm{~m}), 2181(\mathrm{w}), 1464(\mathrm{w}), 1434(\mathrm{w}), 1358(\mathrm{~s})$, $1321(\mathrm{~m}), 1216(\mathrm{~s}), 1198(\mathrm{~s}), 1144(\mathrm{~s}), 1099(\mathrm{~m}), 1079(\mathrm{~m}), 988(\mathrm{~m}), 965(\mathrm{~m}), 883(\mathrm{~m}), 853(\mathrm{~m}), 743(\mathrm{~m})$, 708 (s), 672 (s); HRMS (APCl/QTOF) m/z: $\left[\mathrm{M}+\mathrm{NH}_{4}\right]^{+}$Calcd. for $\mathrm{C}_{27} \mathrm{H}_{44} \mathrm{BF}_{5} \mathrm{NO}_{3} \mathrm{Si}^{+}$564.3098; Found 564.3115 .

\section{Ethyl 2-ethoxy-2-methyl-4-(triisopropylsilyl)but-3-ynoate (5ad)}<smiles>CCOC(=O)C(C)(C#C[Pb])C(=O)OCC</smiles>

Following general procedure $B$, starting from ethanol (4a) $(70.0 \mu \mathrm{L}, 1.20 \mathrm{mmol}),((3,3-$ bis(trifluoromethyl)-1 $\lambda^{3}$-benzo[d][1,2]iodaoxol-1(3H)-yl)ethynyl)triisopropylsilane (2a) (165 mg, 0.300 $\mathrm{mmol})$, and ethyl 2-diazopropanoate (3k) $(1.0 \mathrm{~mL}, 0.60 \mathrm{mmol}, 0.6 \mathrm{M}$ in DCM), afforded the title compound (5ad) as a colorless oil $(42 \mathrm{mg}, 0.13 \mathrm{mmol}, 43 \%) . \mathrm{R}_{\mathrm{f}}=0.37$ (EtOAc/pentane 3:97), $p$ anisaldehyde; ${ }^{1} \mathrm{H}$ NMR $\left(400 \mathrm{MHz}, \mathrm{CDCl}_{3}\right) \delta 4.34-4.12\left(\mathrm{~m}, 2 \mathrm{H},(\mathrm{O}) \mathrm{COCH}_{2}\right), 3.81(\mathrm{dq}, J=8.7,7.1 \mathrm{~Hz}, 1 \mathrm{H}$, $\left.\mathrm{OCH}_{2} \mathrm{CH}_{3}\right), 3.59\left(\mathrm{dq}, J=8.9,7.0 \mathrm{~Hz}, 1 \mathrm{H}, \mathrm{OCH}_{2} \mathrm{CH}_{3}\right), 1.67\left(\mathrm{~s}, 3 \mathrm{H}, \mathrm{CH}_{3}\right), 1.29\left(\mathrm{t}, J=7.1 \mathrm{~Hz}, 3 \mathrm{H},(\mathrm{O}) \mathrm{COCH}_{2} \mathrm{CH}_{3}\right)$, $1.25\left(\mathrm{t}, J=7.0 \mathrm{~Hz}, 3 \mathrm{H}, \mathrm{OCH}_{2} \mathrm{CH}_{3}\right), 1.08(\mathrm{~s}, 21 \mathrm{H}, \mathrm{TIPS}) ;{ }^{13} \mathrm{C} \mathrm{NMR}\left(101 \mathrm{MHz}, \mathrm{CDCl}_{3}\right) \delta$ 171.0, 104.4, 87.9, 75.0, 62.4, 61.9, 27.7, 18.7, 15.7, 14.2, 11.3; IR $\left(v_{\max }, \mathrm{cm}^{-1}\right) 2943(\mathrm{~m}), 2868(\mathrm{~m}), 1748(\mathrm{~s}), 1460(\mathrm{~m}), 1247$ (m), $1196(\mathrm{~m}), 1124(\mathrm{~s}), 1065(\mathrm{~m}), 881(\mathrm{~m}), 670(\mathrm{~s})$; HRMS (ESI/QTOF) m/z: [M+Na] ${ }^{+}$Calcd. for $\mathrm{C}_{18} \mathrm{H}_{34} \mathrm{NaO}_{3} \mathrm{Si}^{+}$349.2169; Found 349.2172. 


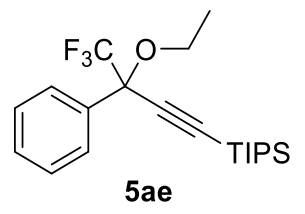

Following general procedure $B$, starting from ethanol (4a) $(70.0 \mu \mathrm{L}, 1.20 \mathrm{mmol}), \quad((3,3-$ bis(trifluoromethyl)-1 $\lambda^{3}$-benzo[d][1,2]iodaoxol-1(3H)-yl)ethynyl)triisopropylsilane (2a) (165 mg, 0.300 $\mathrm{mmol})$, and (1-diazo-2,2,2-trifluoroethyl)benzene (3I) $(1.0 \mathrm{~mL}, 0.60 \mathrm{mmol}, 0.6 \mathrm{M}$ in DCM), afforded the title compound (5ae) as a colorless oil $(66 \mathrm{mg}, 0.17 \mathrm{mmol}, 57 \%) . R_{f}=0.65$ (EtOAc/pentane 3:97), $p$ anisaldehyde; ${ }^{1} \mathrm{H}$ NMR $\left(400 \mathrm{MHz}, \mathrm{CDCl}_{3}\right) \delta 7.78-7.69(\mathrm{~m}, 2 \mathrm{H}, \mathrm{ArH}), 7.45-7.37(\mathrm{~m}, 3 \mathrm{H}, \mathrm{ArH}), 3.83$ (dq, $\left.J=8.9,7.0 \mathrm{~Hz}, 1 \mathrm{H}, \mathrm{OCH}_{2} \mathrm{CH}_{3}\right), 3.44\left(\mathrm{dq}, J=9.0,7.0 \mathrm{~Hz}, 1 \mathrm{H}, \mathrm{OCH}_{2} \mathrm{CH}_{3}\right), 1.28\left(\mathrm{t}, J=7.0 \mathrm{~Hz}, 3 \mathrm{H}, \mathrm{OCH}_{2} \mathrm{CH}_{3}\right)$, $1.21-0.95(\mathrm{~m}, 21 \mathrm{H}, \mathrm{TIPS}) ;{ }^{13} \mathrm{C} \mathrm{NMR}\left(101 \mathrm{MHz}, \mathrm{CDCl}_{3}\right) \delta 134.4,129.6,128.3,128.3,123.1$ (q, $J=285.2$ $\mathrm{Hz}), 99.5,93.2,79.5(\mathrm{q}, J=31.1 \mathrm{~Hz}), 61.7,18.7,15.4,11.3 ;{ }^{19} \mathrm{~F} \mathrm{NMR}\left(376 \mathrm{MHz}, \mathrm{CDCl}_{3}\right) \delta-78.7 ; \mathrm{IR}\left(\mathrm{v}_{\max }\right.$, $\left.\mathrm{cm}^{-1}\right) 2947(\mathrm{~m}), 2869(\mathrm{~m}), 1460(\mathrm{~m}), 1272(\mathrm{~m}), 1180(\mathrm{~s}), 1120(\mathrm{~m}), 1068(\mathrm{~s}), 952(\mathrm{~m}), 884(\mathrm{~m}), 763(\mathrm{~m})$, $708(\mathrm{~m}), 672$ (s); HRMS (ESI/QTOF) m/z: [M+Ag] $]^{+}$Calcd. for $\mathrm{C}_{21} \mathrm{H}_{31} \mathrm{AgF}_{3} \mathrm{OSi}^{+}$491.1142; Found 491.1136.

\section{3-Ethoxy-3-((triisopropylsilyl)ethynyl)dihydrofuran-2(3H)-one (5af)}

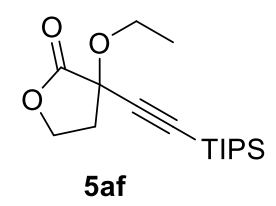

Following general procedure $B$, starting from ethanol (4a) $(70.0 \mu \mathrm{L}, 1.20 \mathrm{mmol}),((3,3-$ bis(trifluoromethyl)-1 $\lambda^{3}$-benzo[d][1,2]iodaoxol-1(3H)-yl)ethynyl)triisopropylsilane (2a) (165 mg, 0.300 $\mathrm{mmol})$, and 3-diazodihydrofuran-2(3H)-one $(3 \mathrm{~m})(1.0 \mathrm{~mL}, 0.60 \mathrm{mmol}, 0.6 \mathrm{M}$ in DCM), afforded the title compound (5af) as a colorless oil (44 $\mathrm{mg}, 0.14 \mathrm{mmol}, 47 \%) . \mathrm{R}_{\mathrm{f}}=0.26$ (EtOAc/pentane 3:97), $p$ anisaldehyde; ${ }^{1} \mathrm{H}$ NMR $\left(400 \mathrm{MHz}, \mathrm{CDCl}_{3}\right) \delta 4.44-4.27\left(\mathrm{~m}, 2 \mathrm{H}, \mathrm{OCH}_{2} \mathrm{CH}_{2}\right), 3.99-3.81\left(\mathrm{~m}, 2 \mathrm{H}, \mathrm{OCH}_{2} \mathrm{CH}_{3}\right)$, $2.66-2.46\left(\mathrm{~m}, 2 \mathrm{H}, \mathrm{OCH}_{2} \mathrm{CH}_{2}\right), 1.23\left(\mathrm{t}, \mathrm{J}=7.0 \mathrm{~Hz}, 3 \mathrm{H}, \mathrm{OCH}_{2} \mathrm{CH}_{3}\right), 1.13-0.94(\mathrm{~m}, 21 \mathrm{H}, \mathrm{TIPS}) ;{ }^{13} \mathrm{C} \mathrm{NMR}$ $\left(101 \mathrm{MHz}, \mathrm{CDCl}_{3}\right) \delta 171.8,100.7,91.9,73.4,65.4,62.4,38.9,18.7,15.5,11.2 ; \mathrm{IR}\left(\mathrm{v}_{\max }, \mathrm{cm}^{-1}\right) 2944(\mathrm{~s})$, $2867(\mathrm{~m}), 2170(\mathrm{w}), 1787(\mathrm{~s}), 1463(\mathrm{~m}), 1377(\mathrm{~m}), 1224(\mathrm{~m}), 1155(\mathrm{~s}), 1060(\mathrm{~s}), 1026(\mathrm{~s}), 884(\mathrm{~m}), 764$ (m), 671 (s); HRMS (APCl/QTOF) m/z: [M+H] ${ }^{+}$Calcd. for $\mathrm{C}_{17} \mathrm{H}_{31} \mathrm{O}_{3} \mathrm{Si}^{+}$311.2037; Found 311.2030.

\section{Ethyl 2-((triisopropylsilyl)ethynyl)tetrahydro-2H-pyran-2-carboxylate (5ag)}

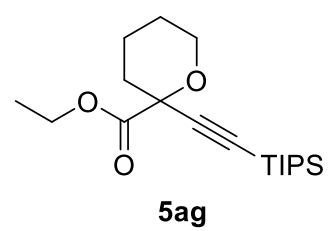

In a $\mathrm{N}_{2}$ filled glovebox, an oven-dried $10 \mathrm{~mL}$ microwave vial was charged with $\mathrm{Cu}\left(\mathrm{CH}_{3} \mathrm{CN}_{4}\right)_{4} \mathrm{PF}_{6}(11.2 \mathrm{mg}$, $30.0 \mu \mathrm{mol}, \quad 0.10 \quad$ equiv.) and ((3,3-bis(trifluoromethyl)-1 $\lambda^{3}$-benzo[d][1,2]iodaoxol- $1(3 H)$ yl)ethynyl)triisopropylsilane (2a) $(165 \mathrm{mg}, 0.300 \mathrm{mmol})$. To the resulting solution was added a $0.6 \mathrm{M}$ solution of ethyl 2-diazo-6-hydroxyhexanoate (3n) $(0.60 \mathrm{mmol}, 2.00$ equiv.) in dry DCM in $1 \mathrm{~h}$ via seringe pump at $25{ }^{\circ} \mathrm{C}$. At the end of the addition, the reaction was continued for $1 \mathrm{~h}$ at the same temperature. The solvent was removed under reduced pressure and the resulting crude product was purified by column chromatography (EtOAc/pentane) directly without further work-up, affording the title compound (5ag) as a colorless oil $(21 \mathrm{mg}, 62 \mu \mathrm{mol}, 21 \%) . \mathrm{R}_{\mathrm{f}}=0.17$ (EtOAc/pentane 3:97), $p$ anisaldehyde; ${ }^{1} \mathrm{H}$ NMR $\left(400 \mathrm{MHz}, \mathrm{CDCl}_{3}\right) \delta 4.26$ (qq, $\left.J=7.1,3.6 \mathrm{~Hz}, 2 \mathrm{H}, \mathrm{OCH}_{2} \mathrm{CH}_{3}\right), 4.09-3.96(\mathrm{~m}, 1 \mathrm{H}$, $\left.\mathrm{OCH}_{2}\right), 3.96-3.85\left(\mathrm{~m}, 1 \mathrm{H}, \mathrm{OCH}_{2}\right), 2.09-2.00(\mathrm{~m}, 1 \mathrm{H}, \mathrm{CH}$-aliphatic $), 2.00-1.69(\mathrm{~m}, 3 \mathrm{H}, \mathrm{CH}$-aliphatic $), 1.67-$ $1.39\left(\mathrm{~m}, 2 \mathrm{H}, \mathrm{CH}\right.$-aliphatic), $1.29\left(\mathrm{t}, J=7.2 \mathrm{~Hz}, 3 \mathrm{H}, \mathrm{OCH}_{2} \mathrm{CH}_{3}\right), 1.16-0.84(\mathrm{~m}, 21 \mathrm{H}, \mathrm{TIPS}) ;{ }^{13} \mathrm{C} \mathrm{NMR}(101 \mathrm{MHz}$, 
$\left.\mathrm{CDCl}_{3}\right) \delta 169.7,102.9,89.4,74.4,64.3,62.0,34.6,25.0,20.0,18.7,14.2,11.3 ; \mathrm{IR}\left(\mathrm{v}_{\max }, \mathrm{cm}^{-1}\right) 2943(\mathrm{~s})$, $2865(\mathrm{~s}), 2166(\mathrm{w}), 1762(\mathrm{~s}), 1742(\mathrm{~s}), 1464(\mathrm{~m}), 1289(\mathrm{~m}), 1255(\mathrm{~s}), 1203(\mathrm{~s}), 1149(\mathrm{~s}), 1095(\mathrm{~m}), 1066$ (s), $1018(\mathrm{~s}), 920(\mathrm{~m}), 882(\mathrm{~s}), 759(\mathrm{~m}), 676(\mathrm{~s}), 660(\mathrm{~s})$; HRMS (ESI/QTOF) m/z: [M+Na] $]^{+}$Calcd. for $\mathrm{C}_{19} \mathrm{H}_{34} \mathrm{NaO}_{3} \mathrm{Si}^{+}$361.2169; Found 361.2174.

2-(4-Bromophenyl)-8-(1,3-dioxoisoindolin-2-yl)-1,1,1-trifluorooct-3-yn-2-yl carbamate (5ah)

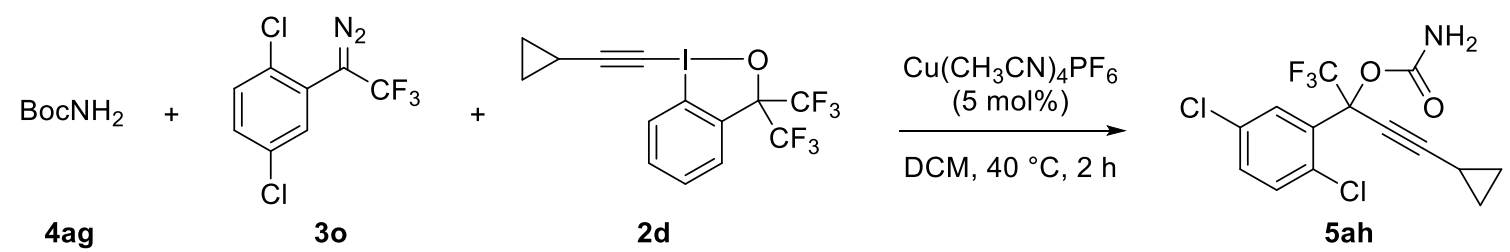

In a $\mathrm{N}_{2}$ filled glovebox, an oven-dried $10 \mathrm{~mL}$ microwave vial was charged with $\mathrm{Cu}\left(\mathrm{CH}_{3} \mathrm{CN}\right)_{4} \mathrm{PF}_{6}(5.59 \mathrm{mg}$, $15.0 \mu \mathrm{mol}, \quad 0.05 \quad$ equiv.), 1 -(cyclopropylethynyl)-3,3-bis(trifluoromethyl)-1,3-dihydro- $1 \lambda^{3}$ benzo[d][1,2]iodaoxole (2d) $(130 \mathrm{mg}, 0.300 \mathrm{mmol}, 1.00$ equiv.) and tert-butyl carbamate (4ag) (45.7 $\mathrm{mg}, 0.390 \mathrm{mmol}, 1.30$ equiv.). The vial was capped, removed from the glovebox and dry DCM (5.35 $\mathrm{mL}$ ) was added. To the resulting solution was added a $0.6 \mathrm{M}$ solution of 1,4-dichloro-2-(1-diazo-2,2,2trifluoroethyl)benzene (3o) $(0.65 \mathrm{~mL}, 0.39 \mathrm{mmol}, 1.30$ equiv.) in dry DCM in $1 \mathrm{~h}$ via seringe pump at 25 ${ }^{\circ} \mathrm{C}$. At the end of the addition, the reaction was continued for $1 \mathrm{~h}$ at the same temperature. The solvent was removed under reduced pressure and the resulting crude product was purified by column chromatography, using DCM as eluent, directly without further work-up affording the title compound (5ah) as a white solid (65 mg, $0.19 \mathrm{mmol}, 62 \%) .{ }^{1} \mathrm{H}$ NMR $\left(400 \mathrm{MHz}, \mathrm{CDCl}_{3}\right) \delta 7.88(\mathrm{~d}, J=2.3 \mathrm{~Hz}, 1 \mathrm{H}$, $\operatorname{ArH}), 7.33-7.29(\mathrm{~m}, 1 \mathrm{H}, \mathrm{ArH}), 7.29-7.24(\mathrm{~m}, 1 \mathrm{H}, \mathrm{ArH}), 4.88\left(\mathrm{br} \mathrm{s}, 2 \mathrm{H}, \mathrm{NH}_{2}\right), 1.42(\mathrm{tt}, J=8.2,5.2 \mathrm{~Hz}$, $1 \mathrm{H}, \mathrm{CH}$-cyclopropyl), $0.94-0.83\left(\mathrm{~m}, 4 \mathrm{H}, \mathrm{CH}\right.$-cyclopropyl); ${ }^{13} \mathrm{C} \mathrm{NMR}\left(101 \mathrm{MHz}, \mathrm{CDCl}_{3}\right) 152.5,133.3,132.8,132.1$, $131.9,131.1,130.7,122.5$ ( $q, J=285.8 \mathrm{~Hz}), 95.3,77.9$ (q $J=33.3 \mathrm{~Hz}), 67.1,8.8,8.7,-0.3 ;{ }^{19} \mathrm{~F}$ NMR $(376$ $\left.\mathrm{MHz}, \mathrm{CDCl}_{3}\right) \delta-77.5$. The values of the NMR spectra are in accordance with reported literature data. ${ }^{1}$

2-(4,4,4-Trifluoro-3-(4-methoxyphenethoxy)-3-(3-(trifluoromethyl)phenyl)but-1-yn-1-yl)thiophene (5ai)<smiles>COc1ccc(CCOC(C#Cc2cccs2)(c2cccc(C(F)(F)F)c2)C(F)(F)F)cc1</smiles>

Following general procedure $B$, starting from 4-methoxyphenethyl alcohol (4ae) (183 mg, $1.20 \mathrm{mmol}$ ), 1-(thiophen-2-ylethynyl)-3,3-bis(trifluoromethyl)-1 $\lambda^{3}$-benzo[d][1,2]iodaoxole (2i) (143 $\mathrm{mg}, 0.300$ $\mathrm{mmol}$ ), and 1-(1-diazo-2,2,2-trifluoroethyl)-3-(trifluoromethyl)benzene $(3 \mathrm{p})(1.0 \mathrm{~mL}, 0.60 \mathrm{mmol}, 0.60$ $\mathrm{M}$ in $\mathrm{DCM}$ ), afforded the title compound (5ai) as an unseparable mixture with the corresponding $\mathrm{O}-\mathrm{H}$ insertion product. The yield was estimated to be $73 \%$ by ${ }^{19} \mathrm{~F}$ NMR spectroscopy. A pure analytical sample was isolated by PTLC using toluene/acetone 95:5 as eluent. $R_{f}=0.31$ (EtOAc/pentane 3:97), $p$ anisaldehyde; ${ }^{1} \mathrm{H}$ NMR $\left(400 \mathrm{MHz}, \mathrm{CDCl}_{3}\right) \delta 7.88(\mathrm{~s}, 1 \mathrm{H}, \mathrm{ArH}), 7.75(\mathrm{~d}, J=7.9 \mathrm{~Hz}, 1 \mathrm{H}, \mathrm{ArH}), 7.69-7.63$ $(\mathrm{m}, 1 \mathrm{H}, \operatorname{ArH}), 7.48(\mathrm{t}, J=7.8 \mathrm{~Hz}, 1 \mathrm{H}, \mathrm{ArH}), 7.41-7.36(\mathrm{~m}, 2 \mathrm{H}, \mathrm{ArH}), \operatorname{ArH}, 7.16-7.10(\mathrm{~m}, 2 \mathrm{H}, \operatorname{ArH}), 7.05$ (dd, $J=5.1,3.7 \mathrm{~Hz}, 1 \mathrm{H}, \mathrm{ArH}), 6.86-6.80(\mathrm{~m}, 2 \mathrm{H}, \mathrm{ArH}), 4.04-3.95\left(\mathrm{~m}, 1 \mathrm{H}, \mathrm{OCH}_{2}\right), 3.79\left(\mathrm{~s}, 3 \mathrm{H}, \mathrm{OCH}_{3}\right)$, $3.59-3.51\left(\mathrm{~m}, 1 \mathrm{H}, \mathrm{OCH}_{2}\right), 2.94\left(\mathrm{t}, J=6.9 \mathrm{~Hz}, 2 \mathrm{H}, \mathrm{CH}_{2} \mathrm{Ar}\right) ;{ }^{13} \mathrm{C} \mathrm{NMR}\left(101 \mathrm{MHz}, \mathrm{CDCl}_{3}\right) \delta 158.4,135.4$, $134.2,131.7,131.0$ (q, $J=32.7 \mathrm{~Hz}), 130.4,130.2,129.1,129.0,127.4,126.7$ (q, $J=3.1 \mathrm{~Hz}), 125.2(q, J=$ $3.5 \mathrm{~Hz}$ ), $123.9(q, J=272.3 \mathrm{~Hz}), 122.8(q, J=285.9 \mathrm{~Hz}), 120.5,114.0,84.8,84.7,79.5(q, J=31.7 \mathrm{~Hz})$, 67.6, 55.4, 35.5; ${ }^{19} \mathrm{~F} \mathrm{NMR}\left(376 \mathrm{MHz}, \mathrm{CDCl}_{3}\right) \delta-62.6,-78.2 ; \mathrm{IR}\left(\mathrm{v}_{\max }, \mathrm{cm}^{-1}\right) 2931(\mathrm{w}), 2226(\mathrm{w}), 1515(\mathrm{~m})$,

${ }^{1}$ D. Dai, X. Long, A. Kulesza, J. Reichwagen, B. Luo and Y. Guo (Lonza Ltd), PCT Int. Appl. WO2012097510, 2012. 
$1329(\mathrm{~m}), 1252(\mathrm{~m}), 1178(\mathrm{~s}), 1130(\mathrm{~s}), 1082(\mathrm{~m}), 1039(\mathrm{~m}), 910(\mathrm{w}), 815(\mathrm{w}), 713(\mathrm{~m})$; HRMS (ESI/QTOF) $\mathrm{m} / \mathrm{z}:[\mathrm{M}+\mathrm{H}]^{+}$Calcd. for $\mathrm{C}_{24} \mathrm{H}_{19} \mathrm{~F}_{6} \mathrm{O}_{2} \mathrm{~S}^{+}$485.1004; Found 485.1006.

\section{3-((2-([1,1'-Biphenyl]-4-yl)-1,1,1-trifluoro-5,5-dimethylhex-3-yn-2-yl)oxy)propanenitrile (5aj)}

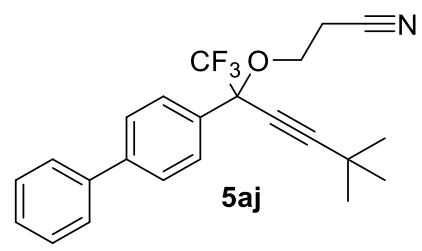

Following general procedure $B$, starting from 3-hydroxypropionitrile (4af) $(81.0 \mu \mathrm{L}, 1.20 \mathrm{mmol}), 1-(3,3-$ dimethylbut-1-yn-1-yl)-3,3-bis(trifluoromethyl)-1,3-dihydro-1 $\lambda^{3}$-benzo[d][1,2]iodaoxole (2j) (135 mg, $0.300 \mathrm{mmol})$, and 4-(1-diazo-2,2,2-trifluoroethyl)-1,1'-biphenyl (3q) $(1.0 \mathrm{~mL}, 0.60 \mathrm{mmol}, 0.6 \mathrm{M}$ in DCM), afforded the title compound 5 aj as a colorless oil $(68 \mathrm{mg}, 0.18 \mathrm{mmol}, 59 \%) . \mathrm{R}_{\mathrm{f}}=0.24$ (EtOAc/pentane 5:95), $p$-anisaldehyde; ${ }^{1} \mathrm{H} \mathrm{NMR}\left(400 \mathrm{MHz}, \mathrm{CDCl}_{3}\right) \delta 7.83-7.75(\mathrm{~m}, 2 \mathrm{H}, \mathrm{ArH}), 7.69-7.57$ $(\mathrm{m}, 4 \mathrm{H}, \mathrm{ArH}), 7.51-7.43(\mathrm{~m}, 2 \mathrm{H}, \mathrm{ArH}), 7.42-7.35(\mathrm{~m}, 1 \mathrm{H}, \mathrm{ArH}), 3.96\left(\mathrm{dt}, J=9.4,6.1 \mathrm{~Hz}, 1 \mathrm{H}, \mathrm{OCH}_{2}\right), 3.67$ (ddd, $\left.J=9.4,7.5,6.1 \mathrm{~Hz}, 1 \mathrm{H}, \mathrm{OCH}_{2}\right), 2.83-2.63\left(\mathrm{~m}, 2 \mathrm{H}, \mathrm{CH}_{2} \mathrm{CN}\right), 1.37(\mathrm{~s}, 9 \mathrm{H}, t \mathrm{Bu}) ;{ }^{13} \mathrm{C} N M R(101 \mathrm{MHz}$, $\left.\mathrm{CDCl}_{3}\right) \delta 142.9,140.3,132.7,129.0,128.7,127.9,127.3,127.3,122.9$ (q, $J=285.0 \mathrm{~Hz}$ ), 117.4, 101.4, $79.4(\mathrm{q}, J=31.8 \mathrm{~Hz}), 70.8,60.4,30.7,28.0,19.0 ;{ }^{19} \mathrm{~F} \mathrm{NMR}\left(376 \mathrm{MHz}, \mathrm{CDCl}_{3}\right) \delta-78.84 ; \mathrm{IR}\left(\mathrm{v}_{\max }, \mathrm{cm}^{-1}\right) 2975$ $(\mathrm{w}), 2251(\mathrm{w}), 1487(\mathrm{~m}), 1290(\mathrm{~m}), 1255(\mathrm{~m}), 1175(\mathrm{~s}), 1090(\mathrm{~s}), 1007(\mathrm{~m}), 943(\mathrm{~m}), 884(\mathrm{~m}), 836(\mathrm{~m})$, 766 (s), 738 (s), 697 (s); HRMS (ESI/QTOF) m/z: [M+Na] ${ }^{+}$Calcd. for $\mathrm{C}_{23} \mathrm{H}_{22} \mathrm{~F}_{3} \mathrm{NNaO}^{+}$408.1546; Found 408.1546 .

\section{2-(4-Bromophenyl)-8-(1,3-dioxoisoindolin-2-yl)-1,1,1-trifluorooct-3-yn-2-yl carbamate (5ak)}

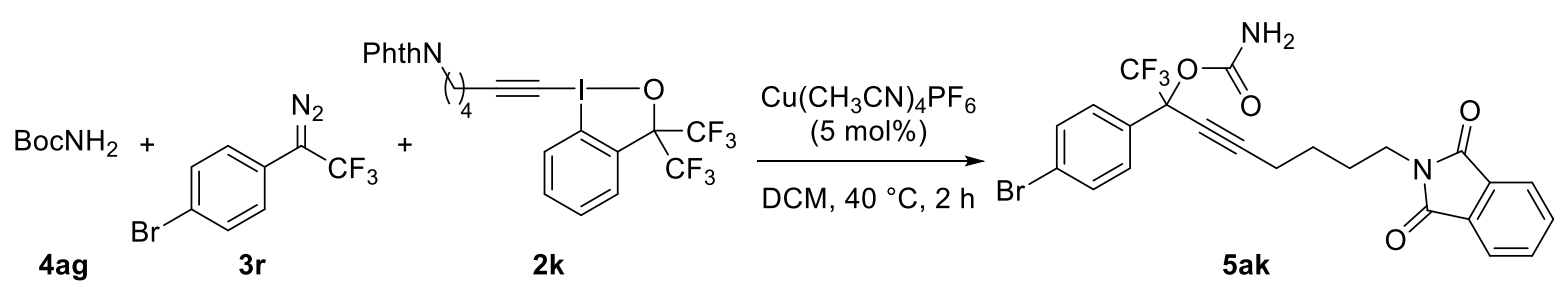

In a $\mathrm{N}_{2}$ filled glovebox, an oven-dried $10 \mathrm{~mL}$ microwave vial was charged with $\mathrm{Cu}\left(\mathrm{CH}_{3} \mathrm{CN}\right)_{4} \mathrm{PF}_{6}(5.59 \mathrm{mg}$, $15.0 \mu \mathrm{mol}, 0.05$ equiv.), 2-(6-(3,3-bis(trifluoromethyl)-1 $\lambda^{3}$-benzo[d][1,2]iodaoxol-1(3H)-yl)hex-5-yn-1$\mathrm{yl}$ )isoindoline-1,3-dione (2k) $(179 \mathrm{mg}, 0.300 \mathrm{mmol}, 1.00$ equiv.) and tert-butyl carbamate (4ag) (45.7 $\mathrm{mg}, 0.390 \mathrm{mmol}, 1.30$ equiv.). The vial was capped, removed from the glovebox and dry DCM (2.35 $\mathrm{mL}$ ) was added. To the resulting solution was added a $0.6 \mathrm{M}$ solution of 1-bromo-4-(1-diazo-2,2,2trifluoroethyl)benzene (3r) $(0.65 \mathrm{~mL}, 0.39 \mathrm{mmol}, 1.30$ equiv.) in dry DCM in $1 \mathrm{~h}$ via seringe pump at 40 ${ }^{\circ} \mathrm{C}$. At the end of the addition, the reaction was continued for $1 \mathrm{~h}$ at the same temperature. The solvent was removed under reduced pressure and the resulting crude product was purified by column chromatography, using EtOAc/pentane 33:67 as eluent, directly without further work-up affording the title compound (5ak) as a white solid $(49 \mathrm{mg}, 90 \mu \mathrm{mol}, 31 \%)$. M.p. $117-118^{\circ} \mathrm{C} ; \mathrm{R}_{\mathrm{f}}=0.54$ (EtOAc/pentane 50:50), $p$-anisaldehyde; ${ }^{1} \mathrm{H}$ NMR (400 MHz, $\left.\mathrm{CDCl}_{3}\right) \delta 7.88-7.79(\mathrm{~m}, 2 \mathrm{H}, \mathrm{ArH}), 7.75-7.67(\mathrm{~m}, 2 \mathrm{H}, \mathrm{ArH})$, $7.56-7.45(\mathrm{~m}, 4 \mathrm{H}, \mathrm{ArH}), 5.00\left(\mathrm{br} \mathrm{s}, 2 \mathrm{H}, \mathrm{NH}_{2}\right), 3.72\left(\mathrm{t}, J=7.0 \mathrm{~Hz}, 2 \mathrm{H}, \mathrm{CH}_{2} \mathrm{~N}\right), 2.44(\mathrm{t}, J=6.9 \mathrm{~Hz}, 2 \mathrm{H}$, $\left.\mathrm{CH}_{2} \mathrm{C} \equiv \mathrm{C}\right), 1.92-1.79\left(\mathrm{~m}, 2 \mathrm{H}, \mathrm{CH}_{2}\right), 1.74-1.61\left(\mathrm{~m}, 2 \mathrm{H}, \mathrm{CH}_{2}\right) ;{ }^{13} \mathrm{C}$ NMR $\left(101 \mathrm{MHz}, \mathrm{CDCl}_{3}\right) \delta$ 168.6, 152.4, $134.1,133.1,132.2,131.7,128.8,124.1,123.4,122.2$ (q, $J=284.2 \mathrm{~Hz}), 91.1,72.7,72.2(\mathrm{q}, J=32.4 \mathrm{~Hz}$ ), 37.5, 27.8, 25.4, 18.5; ${ }^{19} \mathrm{~F}$ NMR $\left(376 \mathrm{MHz}, \mathrm{CDCl}_{3}\right) \delta$-77.5; IR $\left(\mathrm{v}_{\max }, \mathrm{cm}^{-1}\right) 2945(\mathrm{~m}), 2870(\mathrm{~m}), 2358(\mathrm{w})$, $1461(\mathrm{~m}), 1273(\mathrm{~m}), 1180(\mathrm{~s}), 1117$ (s), $1068(\mathrm{~s}), 763(\mathrm{~m}), 711(\mathrm{~m}), 674(\mathrm{~s})$; HRMS (ESI/QTOF) m/z: $[\mathrm{M}+\mathrm{Na}]^{+}$Calcd. for $\mathrm{C}_{23} \mathrm{H}_{18} \mathrm{BrF}_{3} \mathrm{~N}_{2} \mathrm{NaO}_{4}{ }^{+}$545.0294; Found 545.0284. 


\section{Control experiments and mechanistic studies}

a) Sequential addition of the alcohol and the EBX reagent (Scheme 5, Eq. (1)):

In a $\mathrm{N}_{2}$ filled glovebox, an oven-dried $10 \mathrm{~mL}$ microwave vial was charged with $\mathrm{Cu}\left(\mathrm{CH}_{3} \mathrm{CN}\right)_{4} \mathrm{PF}_{6}(3.0 \mathrm{mg}$, $8.0 \mu \mathrm{mol}, 0.20$ equiv.). The vial was capped, removed from the glovebox and $\mathrm{CD}_{2} \mathrm{Cl}_{2}(0.80 \mathrm{~mL})$ was added, followed by EtOH (4a) $(19 \mu \mathrm{L}, 0.32 \mathrm{mmol}, 8.00$ equiv.). Ethyl diazoacetate (3a) (19 $\mu \mathrm{L}, 0.16$ mmol, 4.00 equiv., $87 \% \mathrm{wt}$ in $\mathrm{DCM}$ ) was added dropwise and the resulting reaction mixture was stirred at room temperature for $1 \mathrm{~h}$. After this time, an aliquot of the solution $(0.40 \mathrm{~mL})$ was taken and a ${ }^{1} \mathrm{H}$ NMR spectrum of the reaction mixture was recorded (c). A solution of TIPS-EBX (2a) $(22.0 \mathrm{mg}, 40.0$ $\mu \mathrm{mol}, 1.00$ equiv.) in $\mathrm{CD}_{2} \mathrm{Cl}_{2}(0.40 \mathrm{~mL})$ was added to the first solution and the reaction was continued for $1 \mathrm{~h}$ at room temperature. $\mathrm{A}^{1} \mathrm{H}$ NMR spectrum was recoreded (d).
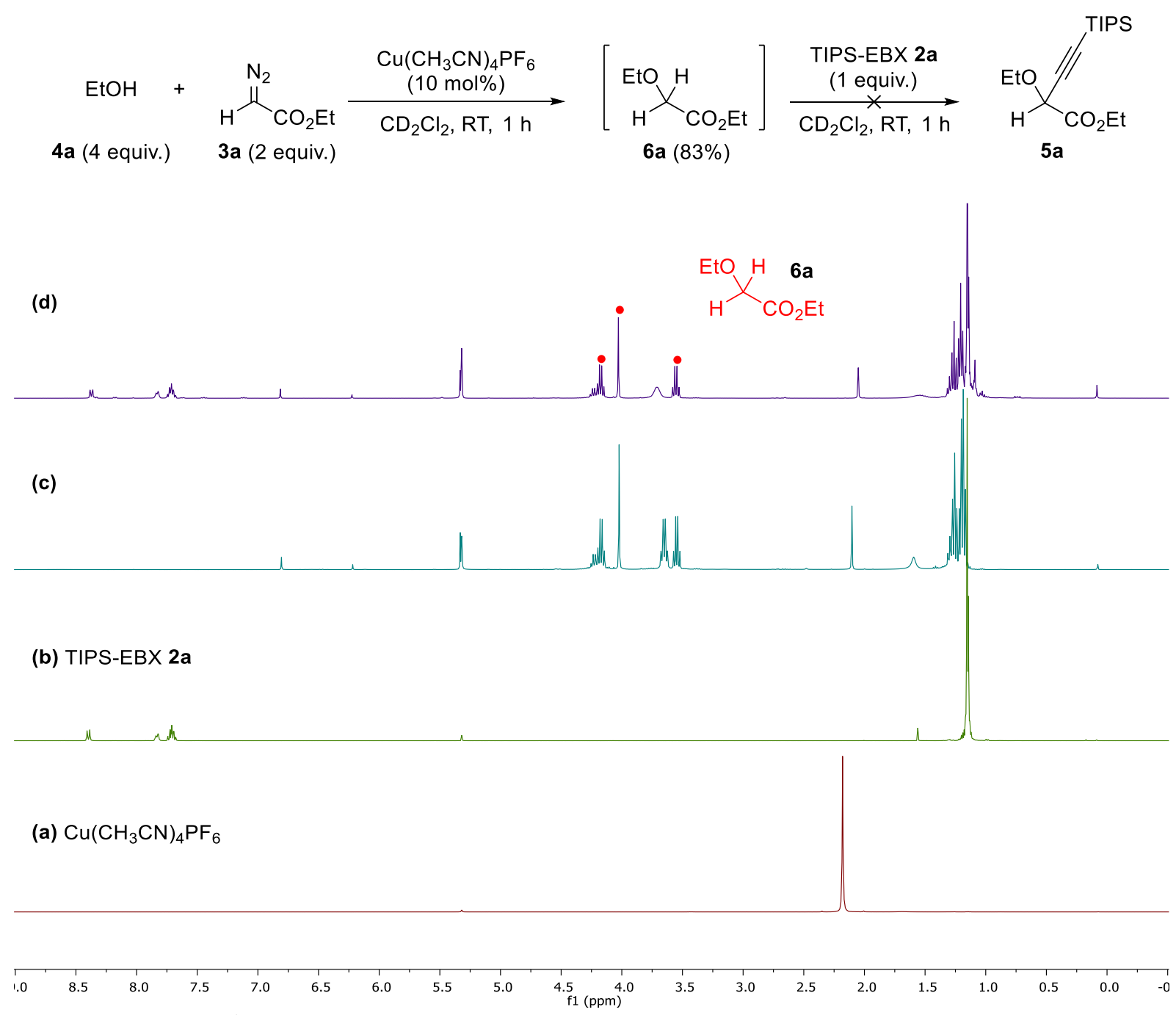

Figure S1: ${ }^{1} \mathrm{H}$ NMR spectra of the sequential addition of EtOH (4a) and TIPS-EBX (2a).

No NMR signal of the desired three-component product 5a appeared after the addition of TIPS-EBX (2a) to the already formed $\mathrm{O}-\mathrm{H}$ insertion product $\mathbf{6 a}$ in presence of the copper catalyst.

b) EBX reagent and diazo compound in presence of copper catalyst (Scheme 5, Eq. (2)):

In a $\mathrm{N}_{2}$ filled glovebox, an oven-dried $10 \mathrm{~mL}$ microwave vial was charged with $\mathrm{Cu}\left(\mathrm{CH}_{3} \mathrm{CN}_{4}\right)_{4} \mathrm{PF}_{6}(3.0 \mathrm{mg}$, $8.0 \mu \mathrm{mol}, 0.10$ equiv.) and TIPS-EBX $2 \mathrm{a}(44 \mathrm{mg}, 80 \mu \mathrm{mol}, 1.00$ equiv.). The vial was capped, removed from the glovebox and $\mathrm{CD}_{2} \mathrm{Cl}_{2}(0.80 \mathrm{~mL})$ was added, followed by the dropwise addition of ethyl diazoacetate (3a) (19 $\mu \mathrm{L}, 0.16 \mathrm{mmol}, 4.00$ equiv., $87 \% \mathrm{wt}$ in $\mathrm{DCM})$. Rapid evolution of nitrogen was 
observed to occur. The reaction was continued for $1 \mathrm{~h}$ at room temperature. $A^{1} \mathrm{H}$ NMR spectrum was recorded (c).

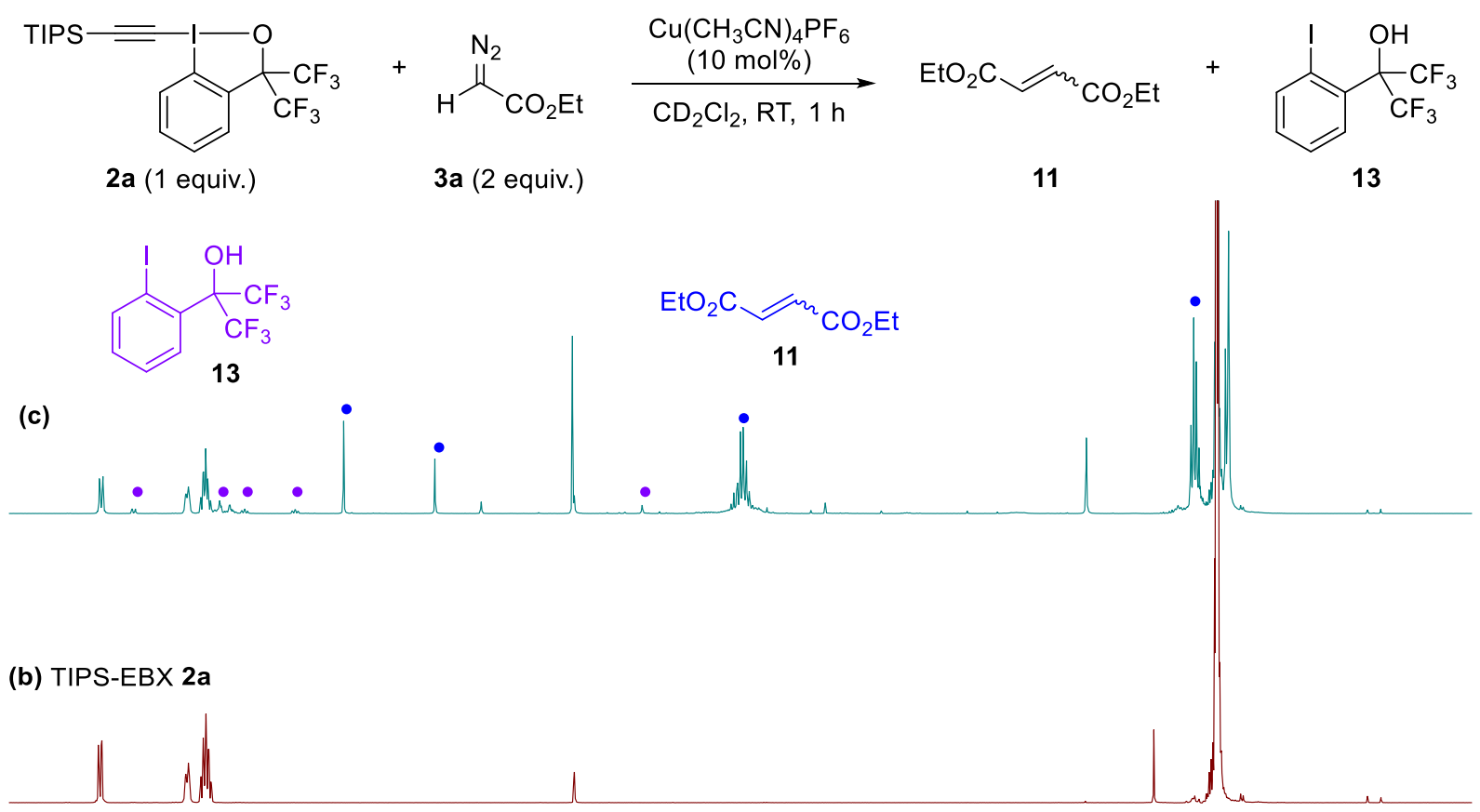

(a) EDA 3a
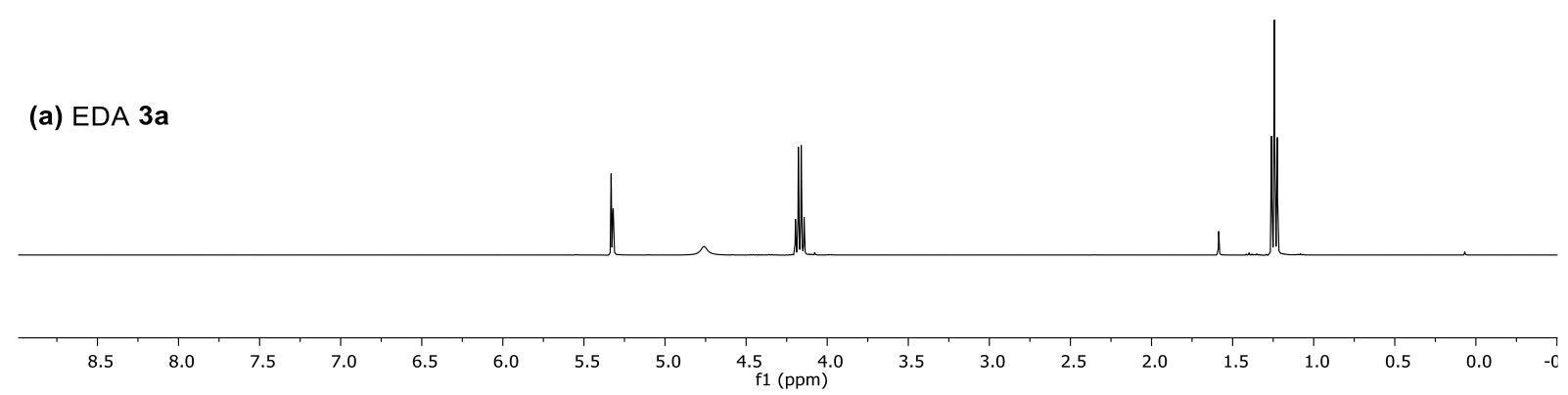

Figure S2: ${ }^{1} \mathrm{H}$ NMR spectra of the dimerization of EDA (3a) in presence of EBX (2a).

There was an obvious dimerization of ethyl diazoacetate (3a) to diethyl fumarate/maleate (9) in absence of alcohol. Approximately $10 \%$ of the EBX reagent $\mathbf{2 a}$ was degraded to the precursor $\mathbf{1 3}$ and other unidentified by-products.

c) ${ }^{1} \mathrm{H}$ and ${ }^{19} \mathrm{~F}$ NMR titration of EBX reagent with $\mathrm{Cu}\left(\mathrm{CH}_{3} \mathrm{CN}\right){ }_{4} \mathrm{PF}_{6}$ :

In a $\mathrm{N}_{2}$ filled glovebox, two differents oven-dried $10 \mathrm{~mL}$ microwave vials were prepared with the following solutions:

(1): $0.1 \mathrm{M}$ solution of TIPS-EBX (2a) (110 mg, $2.00 \mathrm{mmol})$ in $\mathrm{CD}_{2} \mathrm{Cl}_{2}(2.0 \mathrm{~mL})$.

(2): $0.1 \mathrm{M}$ solution of $\mathrm{Cu}\left(\mathrm{CH}_{3} \mathrm{CN}\right)_{4} \mathrm{PF}_{6}(74.5 \mathrm{mg}, 2.00 \mathrm{mmol})$ in $\mathrm{CD}_{2} \mathrm{Cl}_{2}(2.0 \mathrm{~mL})$.

TIPS-EBX (2a) (200 $\mu \mathrm{L}$ of solution (1), $0.02 \mathrm{mmol}, 1.00$ equiv.) was then stirred for $1 \mathrm{~h}$ at room temperature with differents equivalent of $\mathrm{Cu}\left(\mathrm{CH}_{3} \mathrm{CN}\right)_{4} \mathrm{PF}_{6}$ (gradient from $0 \mu \mathrm{L}$ of solution (2), 0.00 mmol, 0.00 equiv. to $600 \mu \mathrm{L}$ of solution (2), $0.06 \mathrm{mmol}, 3.00$ equiv.) All solutions were adjusted with $\mathrm{CD}_{2} \mathrm{Cl}_{2}(600 \mu \mathrm{L}$ to $0 \mu \mathrm{L})$ before the addition of the solution (2) to have $\mathrm{V}_{\text {tot }}=800 \mu \mathrm{L}$.

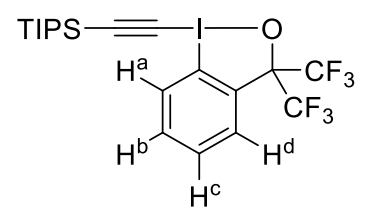

2a
${ }^{1} \mathrm{H}^{\mathrm{a}}: \delta 8.43-8.36(\mathrm{~m}, 1 \mathrm{H})$
${ }^{1} \mathrm{H}^{\mathrm{b}+\mathrm{c}}: \delta 7.76-7.66(\mathrm{~m}, 2 \mathrm{H})$
${ }^{1} \mathrm{H}^{\mathrm{d}}: \delta 7.86-7.79(\mathrm{~m}, 1 \mathrm{H})$
${ }^{19} \mathrm{~F}: \delta 76.5$ 


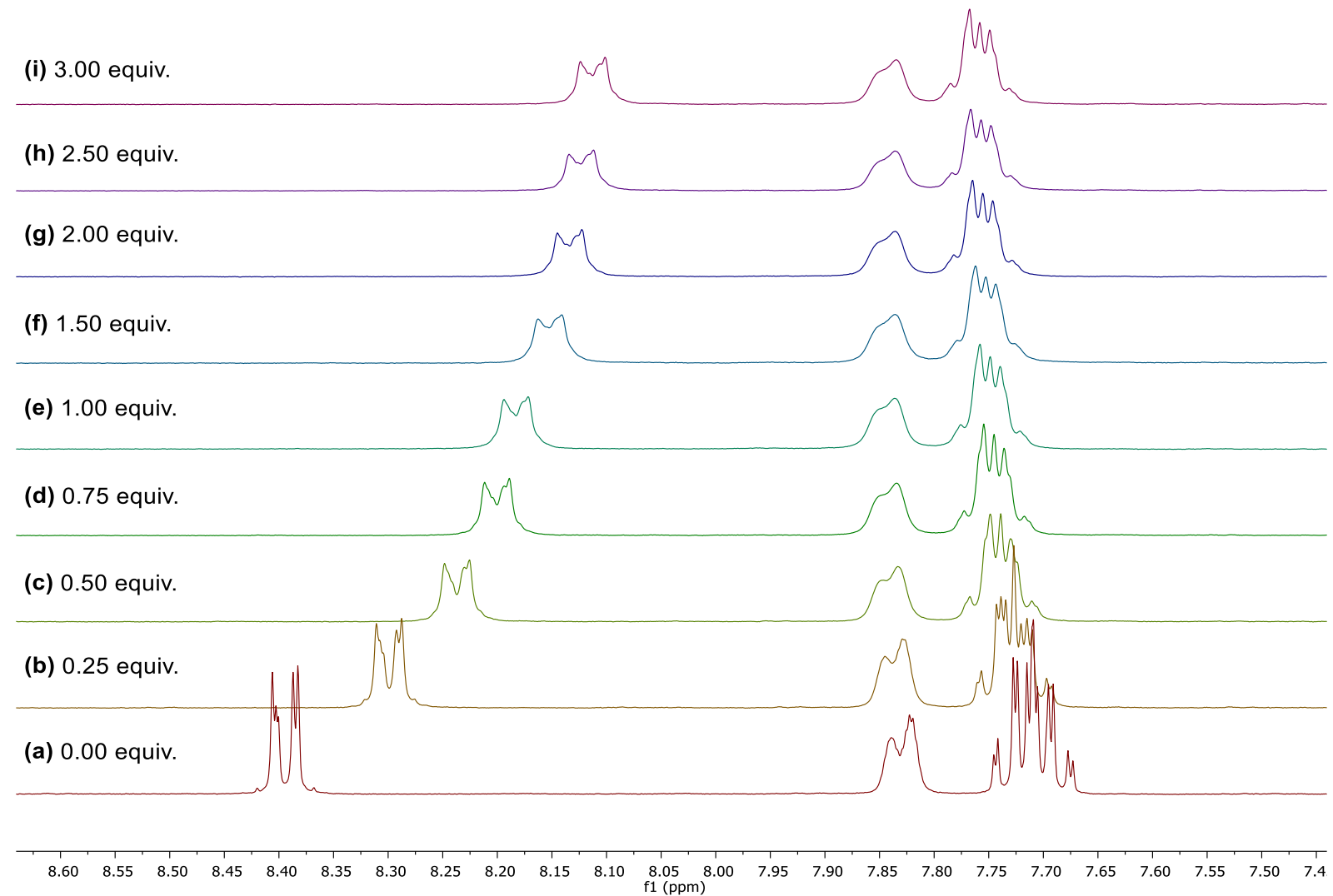

Figure S3: ${ }^{1} \mathrm{H}$ NMR titration of the EBX reagent with $\mathrm{Cu}\left(\mathrm{CH}_{3} \mathrm{CN}\right)_{4} \mathrm{PF}_{6}$.

(i) 3.00 equiv.

(h) 2.50 equiv.

(g) 2.00 equiv.

(f) 1.50 equiv

(e) 1.00 equiv

(d) 0.75 equiv.

(c) 0.50 equiv.

(b) 0.25 equiv

(a) 0.00 equiv.

\begin{tabular}{|c|c|c|c|c|c|c|c|c|c|c|}
\hline-71.5 & -72.0 & -72.5 & -73.0 & -73.5 & -74.0 & f1 (ppm) & -75.0 & -75.5 & -76.0 & -76.5 \\
\hline
\end{tabular}

Figure S4: ${ }^{19} \mathrm{~F} N M R$ titration of the $\mathrm{EBX}$ reagent with $\mathrm{Cu}\left(\mathrm{CH}_{3} \mathrm{CN}\right)_{4} \mathrm{PF}_{6}$. 
Progressive shifts of the aromatic ${ }^{1} \mathrm{H}^{\mathrm{a}}$ were observed upon addition of the copper salt, with a significant diminution after one equivalent. Others ${ }^{1} \mathrm{H}$ signals were less influenced by the presence of the $\mathrm{Cu}$ salt. Minor shift of ${ }^{19} \mathrm{~F}$ signal of $\mathbf{2 a}$ was observed (The new doublet appearing comes from the $\mathrm{PF}_{6}{ }^{-}$).

d) ${ }^{13} \mathrm{C}$ NMR spectrum of the complexation of the EBX reagent with $\mathrm{Cu}\left(\mathrm{CH}_{3} \mathrm{CN}\right){ }_{4} \mathrm{PF}_{6}$ :

Samples (a) (0.00 equiv.) and (e) (1.00 equiv.) from the titration experiment were submitted to ${ }^{13} \mathrm{C}$ NMR analysis.
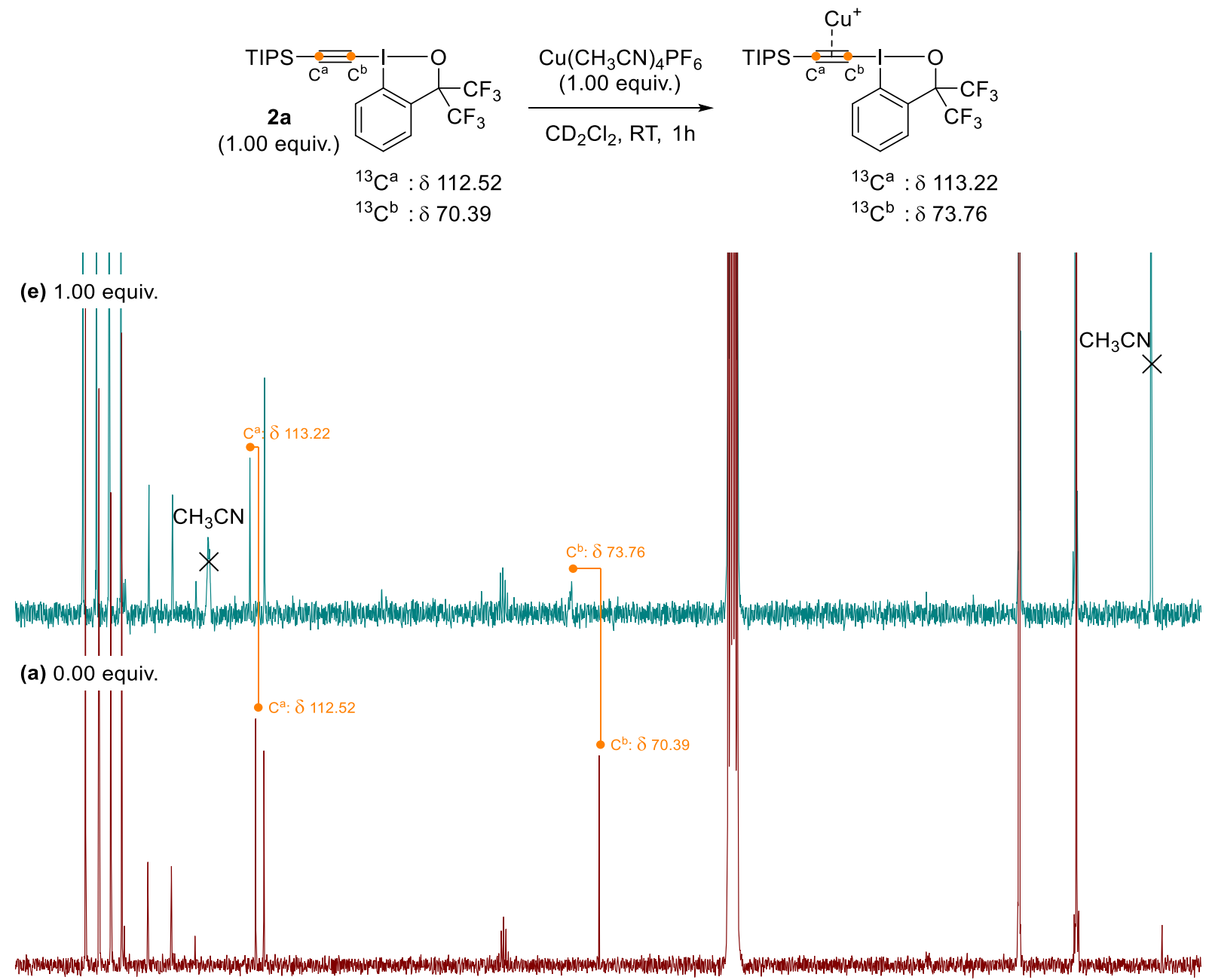

$\begin{array}{lllllllllllllll}140 & 135 & 130 & 125 & 120 & 115 & 110 & 105 & 100 & 95 & 90 & 85 & 80 & 75 & 70 \\ \mathrm{f} 1(\mathrm{ppm})\end{array}$

Figure S5: ${ }^{13} \mathrm{C}$ NMR spectrum of the complexation of the $\mathrm{EBX}$ reagent and $\mathrm{Cu}\left(\mathrm{CH}_{3} \mathrm{CN}\right)_{4} \mathrm{PF}_{6}$.

Major changes of ${ }^{13} \mathrm{C}^{\mathrm{a}}(\Delta \delta=0.7 \mathrm{ppm}, 71.1 \mathrm{~Hz})$ and ${ }^{13} \mathrm{C}^{\mathrm{b}}(\Delta \delta=3.4 \mathrm{ppm}, 339.5 \mathrm{~Hz})$ were observed. Other ${ }^{13} \mathrm{C}$ signals remained almost unchanged in presence of the $\mathrm{Cu}$ salt.

e) Mixing EtOH with $\mathrm{Cu}\left(\mathrm{CH}_{3} \mathrm{CN}\right)_{4} \mathrm{PF}_{6}$ :

In a $\mathrm{N}_{2}$ filled glovebox, an oven-dried $10 \mathrm{~mL}$ microwave vial was charged with $\mathrm{Cu}\left(\mathrm{CH}_{3} \mathrm{CN}\right)_{4} \mathrm{PF}_{6}(30 \mathrm{mg}$, $0.080 \mathrm{mmol}, 1.00$ equiv.). The vial was capped, removed from the glovebox and $\mathrm{CD}_{2} \mathrm{Cl}_{2}(0.80 \mathrm{~mL})$ was added, followed by the addition of EtOH (4a) $(19 \mu \mathrm{L}, 0.32 \mathrm{mmol}, 4.00$ equiv.). The resulting reaction mixture was stirred at room temperature for $1 \mathrm{~h} . \mathrm{A}^{1} \mathrm{H}$ NMR spectrum was recoreded (c). The solvent was then removed under reduced pressure and a new ${ }^{1} \mathrm{H}$ NMR spectrum was recorded in $\mathrm{CD}_{2} \mathrm{Cl}_{2}$ (d). 


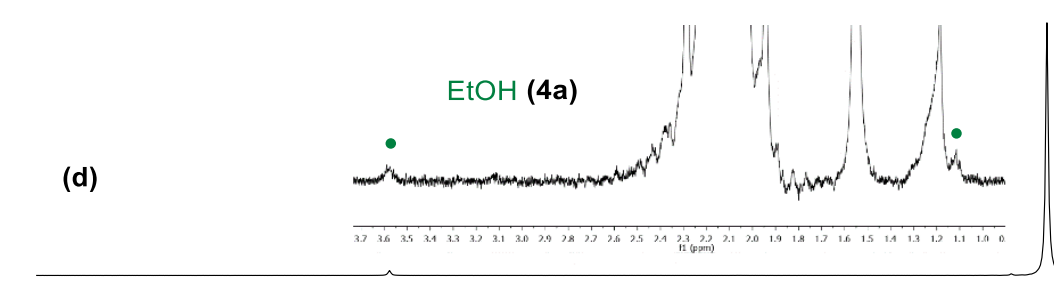

(c)

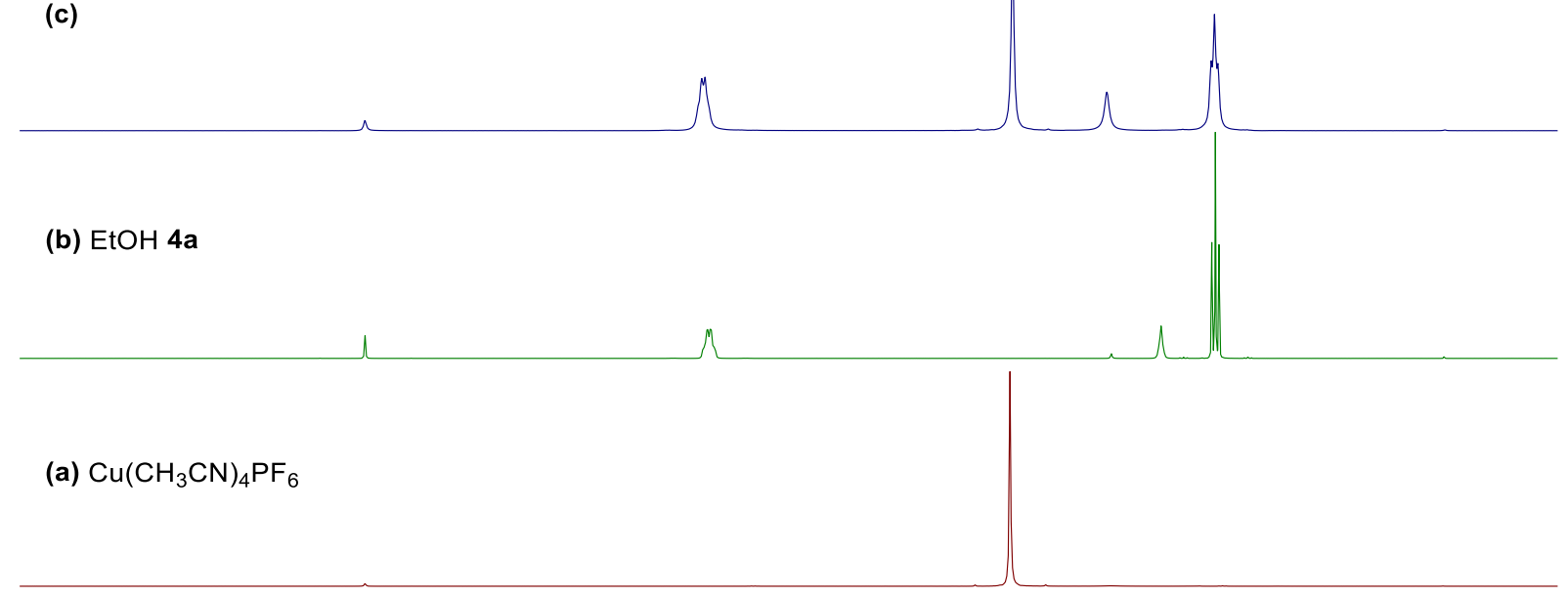

\begin{tabular}{llllllllllllll}
\hline .0 & 6.5 & 6.0 & 5.5 & 5.0 & 4.5 & 4.0 & 3.5 & 1 & 1 \\
$\mathrm{f} 1(\mathrm{ppm})$ & 3.0 & 2.5 & 2.0 & 1.5 & 1.0 & 0.5 & 0.0 & 1
\end{tabular}

Figure S6: ${ }^{1} \mathrm{H}$ NMR spectrum of the complexation of EtOH and $\mathrm{Cu}\left(\mathrm{CH}_{3} \mathrm{CN}\right)_{4} \mathrm{PF}_{6}$.

No shift of ${ }^{1} \mathrm{H}$ signals of ethanol or $\mathrm{Cu}\left(\mathrm{CH}_{3} \mathrm{CN}\right)_{4} \mathrm{PF}_{6}$ were observed. When the reaction mixture was evaporated, the initial copper salt was recovered.

f) Cu-carbene trapping through cyclopropanation (Scheme 5, Eq (3)):

In a $\mathrm{N}_{2}$ filled glovebox, an oven-dried $10 \mathrm{~mL}$ microwave vial was charged with $\mathrm{Cu}\left(\mathrm{CH}_{3} \mathrm{CN}\right)_{4} \mathrm{PF}_{6}(3.0 \mathrm{mg}$, $8.0 \mu \mathrm{mol}, 0.10$ equiv.) and TIPS-EBX $2 \mathrm{a}(44 \mathrm{mg}, 80 \mu \mathrm{mol}, 1.00$ equiv.). The vial was capped, removed from the glovebox and $\mathrm{CD}_{2} \mathrm{Cl}_{2}(0.80 \mathrm{~mL})$ was added, followed by the addition of ethanol (4a) $(19 \mu \mathrm{L}$, $0.32 \mathrm{mmol}, 4.00$ equiv.) and styrene $(37 \mu \mathrm{L}, 0.32 \mathrm{mmol}, 4.00$ equiv.). A solution of ethyl diazoacetate (3a) in DCM (133 $\mu \mathrm{L}, 80 \mu \mathrm{mol}, 1.00$ equiv., $0.6 \mathrm{M})$ was slowly added in $1 \mathrm{~h}$ via seringe pump. At the end of the addition, the reaction was continued $1 \mathrm{~h}$ at room temperature. ${ }^{1} \mathrm{H}$ NMR spectrum of the reaction mixture was recoreded (d). For comparison, control experiments missing the EBX reagent (spectrum (c)), ethanol (spectrum (b)) and EBX + ethanol (spectrum (a)) were done. 


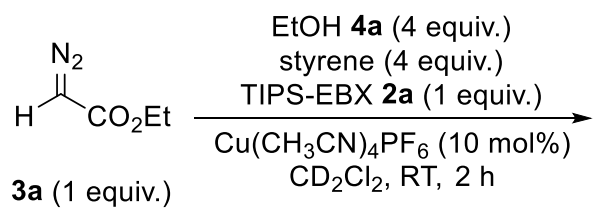

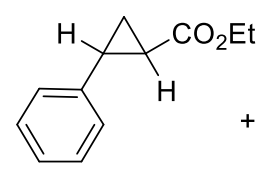

$12(21 \%)$

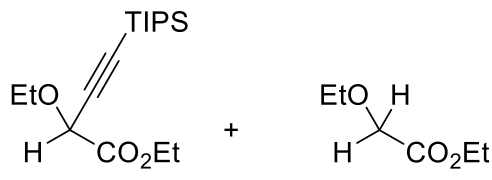

5 a $(62 \%)$

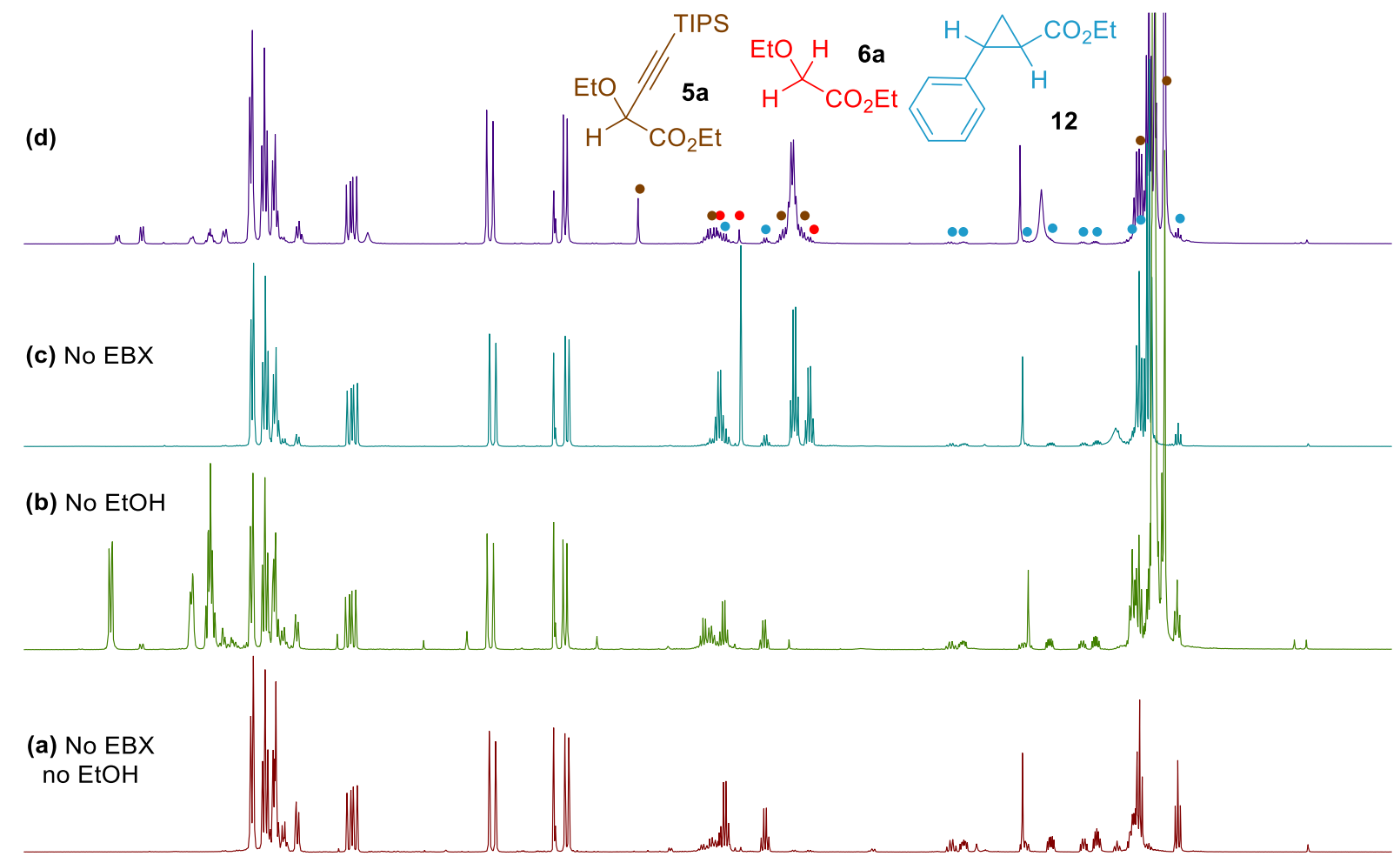

$\begin{array}{lll}1.0 & 8.5 & 8.0 \\ & & \end{array}$

Figure S7: ${ }^{1} \mathrm{H}$ NMR spectrum of the competitive cyclopropanation with styrene.

The three-component product $\mathbf{5}$ a was the major product observed (62\%). The conversion of TIPS-EBX (2a) was $62 \%$. A detectable amount of cyclopropane 12 was observed (21\%). The O-H insertion product 6a was also detected (10\%). In absence of $\mathrm{EtOH}$, cyclopropane $\mathbf{1 2}$ was the major product formed (spectrum (a) and (b)). The $\mathrm{O}-\mathrm{H}$ insertion product 6 a was predominant $(83 \%)$ over the cyclopropane 12 (16\%) when EtOH (4.00 equiv.) and styrene (4.00 equiv.) were in competition (spectrum (c)).

g) Effect of $t$ BuBOX ligand (Scheme 5, Eq (4)):<smiles>FC(F)(F)C(F)(F)OI1C#C[In]C1(C(F)(F)F)C(F)(F)F</smiles>

$2 a$<smiles>CCOC(=O)C=[N+]</smiles>

3a<smiles>OCc1ccccc1</smiles>

4b
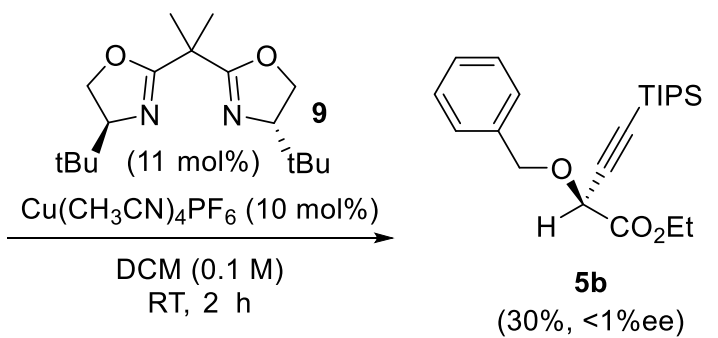

In a $\mathrm{N}_{2}$ filled glovebox, a catalytic solution was prepared by mixing $\mathrm{Cu}\left(\mathrm{CH}_{3} \mathrm{CN}\right)_{4} \mathrm{PF}_{6}(16.8 \mathrm{mg}, 45.0 \mu \mathrm{mol}$, 0.15 equiv.) and (S,S)-(-)-2,2'-isopropylidenebis(4-tert-butyl-2-oxazoline) (9) (14.6 mg, $50.0 \mu \mathrm{mol}, 0.17$ equiv.) in dry DCM (3.00 $\mathrm{mL})$ at room temperature for $1 \mathrm{~h}$.

$2.00 \mathrm{~mL}$ of the catalytic solution was added to an oven-dried $10 \mathrm{~mL}$ microwave vial previously charged with TIPS-EBX 2a (165 mg, $0.3 \mathrm{mmol}, 1.00$ equiv.) under $\mathrm{N}_{2}$ atmosphere followed by benzyl alcohol (4b) 
(124 $\mu \mathrm{L}, 1.20 \mathrm{mmol}, 4.00$ equiv.). To the resulting solution was added ethyl 2-diazoacetate (3a) (1.0 $\mathrm{mL}, 0.60 \mathrm{mmol}, 0.6 \mathrm{M}$ in DCM) in $1 \mathrm{~h}$ via seringe pump at $25^{\circ} \mathrm{C}$. The system was mainted isobaric with a filled balloon with $\mathrm{N}_{2}$. At the end of the addition, the reaction was continued for $1 \mathrm{~h}$ at the same temperature. The solvent was removed under reduced pressure and the resulting crude product was analyzed by ${ }^{1} \mathrm{H}$ NMR. Ethyl 2-(benzyloxy)-4-(triisopropylsilyl)but-3-ynoate (5b) was formed in $30 \%$ yield in the crude reaction mixture. The crude product was purified by column chromatography using EtOAc/pentane 3:97 as eluent to afford ethyl 2-(benzyloxy)-4-(triisopropylsilyl)but-3-ynoate (5b) (23 $\mathrm{mg}, 0.06 \mathrm{mmol}, 20 \%)$ as a colorless oil. Chiral HPLC conditions: ee $=<1 \%$; Chiralpak IA 98:2 Hexane $/ \mathrm{iPrOH}, 1.0 \mathrm{~mL} / \mathrm{min}, 60 \mathrm{~min} . \operatorname{tr}(1)=6.051 \mathrm{~min}$. and $\operatorname{tr}(2)=6.416 \mathrm{~min} . \lambda=254 \mathrm{~cm}^{-1}$.

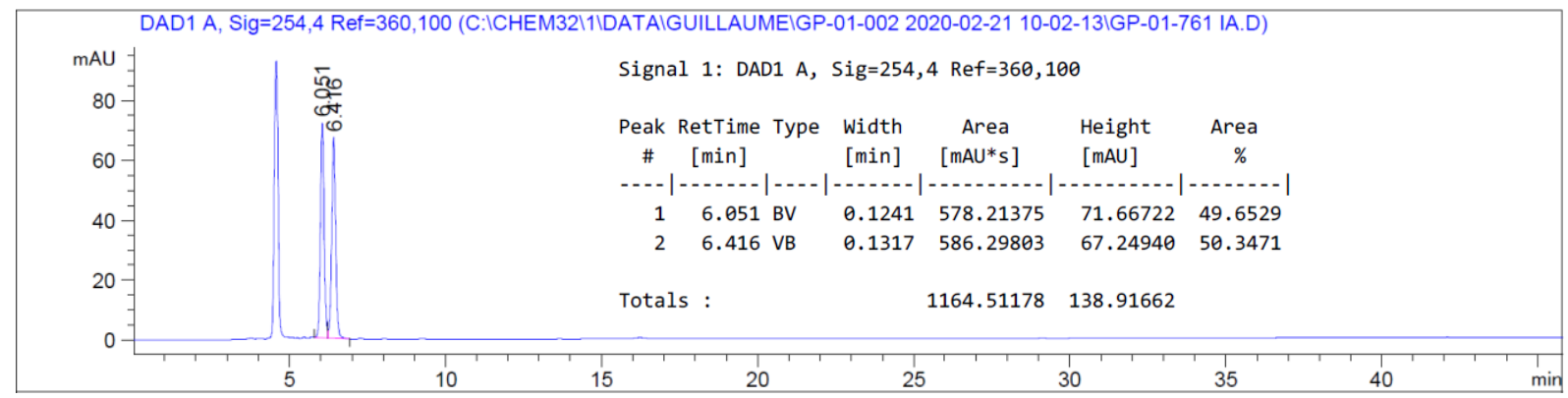

Benzyl alcohol (4b) was used instead of ethanol (4a) to facilitate HPLC analysis of the enantioselectivity. 


\section{Synthesis of diazo-compounds}

CAUTION: Diazo compounds are toxic and potentially explosive and should be handled with care in a well-ventilated hood. ${ }^{2}$

Ethyl 2-diazoacetate (3a) and benzyl 2-diazoacetate (3b) were directly purchased from Sigma Aldrich.<smiles>CCOC(=O)C=[W]</smiles>

3a<smiles>N=CC(=O)OCc1ccccc1</smiles>

$3 b$

\section{p-Tolyl 2-diazoacetate (3c)}

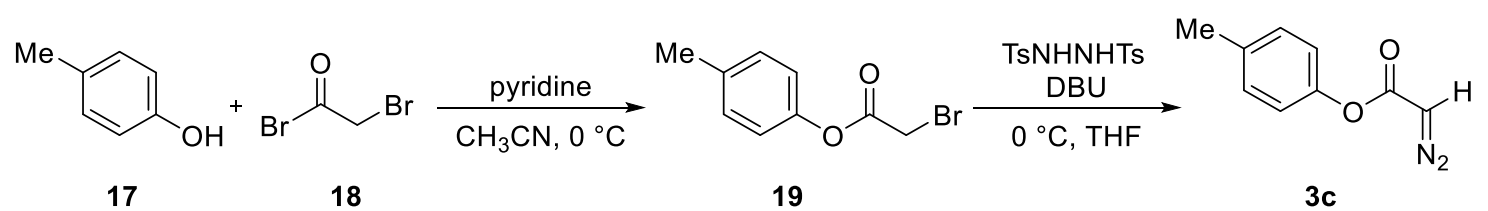

Bromoacetyl bromide (18) $(1.31 \mathrm{~mL}, 15.0 \mathrm{mmol}, 1.50$ equiv) was added to a stirred solution of $p$-cresol (17) $(1.08 \mathrm{~g}, 10.0 \mathrm{mmol}, 1.00$ equiv) and pyridine (1.61 mL, $20.0 \mathrm{mmol}, 2.00$ equiv) in acetonitrile (50 $\mathrm{mL}$ ) at $0{ }^{\circ} \mathrm{C}$ over 10 minutes. The mixture was stirred for further 5 minutes at $0{ }^{\circ} \mathrm{C}$ and then quenched with water $(30 \mathrm{~mL})$. The reaction mixture was extracted with dichloromethane $(3 \times 30 \mathrm{~mL})$. The combined organic layers were washed with brine, dried over $\mathrm{MgSO}_{4}$, filtered and concentrated under reduced pressure. The crude product was purified by column chromatography using EtOAc/pentane 5:95 as eluent to afford $p$-tolyl 2-bromoacetate (19) as a colorless oil $(2.1 \mathrm{~g}, 9.2 \mathrm{mmol}, 92 \%) .{ }^{1} \mathrm{H}$ NMR $\left(400 \mathrm{MHz}, \mathrm{CDCl}_{3}\right) \delta 7.23-7.15(\mathrm{~m}, 2 \mathrm{H}, \mathrm{ArH}), 7.05-6.95(\mathrm{~m}, 2 \mathrm{H}, \mathrm{ArH}), 4.04\left(\mathrm{~s}, 2 \mathrm{H}, \mathrm{CH}_{2}\right), 2.35(\mathrm{~s}, 3 \mathrm{H}$, $\left.\mathrm{CH}_{3}\right) ;{ }^{13} \mathrm{C}$ NMR $\left(100 \mathrm{MHz}, \mathrm{CDCl}_{3}\right) \delta 166.2,148.4,136.2,130.2,120.9,25.7,21.0$. The values of the NMR spectra are in accordance with reported literature data. ${ }^{3}$

Following a reported procedure, $N, N^{\prime}$-Ditosylhydrazine $(3.40 \mathrm{~g}, 10.0 \mathrm{mmol}, 2.00$ equiv) was added to a solution of $p$-tolyl 2-bromoacetate (19) $(1.15 \mathrm{~g}, 5.00 \mathrm{mmol}, 1.00$ equiv) in tetrahydrofuran $(20 \mathrm{~mL})$ and the mixture was cooled to $0{ }^{\circ} \mathrm{C}$. 1,8-Diazabicycloundec-7-ene $(3.8 \mathrm{~mL}, 25 \mathrm{mmol}, 5.0$ equiv) was added dropwise over 20 minutes at $0{ }^{\circ} \mathrm{C}$. The reaction was stirred $2 \mathrm{~h}$ at $0{ }^{\circ} \mathrm{C}$ before being quenched by a saturated aqueous $\mathrm{NaHCO}_{3}$ solution $(30 \mathrm{~mL}$ ). The reaction mixture was extracted with diethyl ether ( 3 x $20 \mathrm{~mL}$ ). The combined organic layers were washed with brine, dried over $\mathrm{MgSO}_{4}$, filtered and concentrated under reduced pressure. The crude product was purified by column chromatography using EtOAc/pentane 5:95 as eluent to afford $p$-tolyl 2-diazoacetate $(3 \mathrm{c})$ as a yellow oil $(0.450 \mathrm{~g}, 2.55$ $\mathrm{mmol}, 51 \%) . \mathrm{R}_{\mathrm{f}}=0.33$ (EtOAc/pentane 5:95), $\mathrm{KMnO}_{4} ;{ }^{1} \mathrm{H} \mathrm{NMR}\left(400 \mathrm{MHz}, \mathrm{CDCl}_{3}\right) \delta 7.20-7.14(\mathrm{~m}, 2 \mathrm{H}$, $\mathrm{ArH}), 7.03$ - 6.98 (refm, 2H, $\mathrm{ArH}), 4.95$ (br s, $\left.1 \mathrm{H}, \mathrm{CHN}_{2}\right), 2.34\left(\mathrm{~s}, 3 \mathrm{H}, \mathrm{CH}_{3}\right) ;{ }^{13} \mathrm{C} \mathrm{NMR}(100 \mathrm{MHz}, \mathrm{CDCl} 3) \delta$ 148.4, 135.7, 130.1, 121.5, 46.9, 21.0; IR $\left(v_{\max }, \mathrm{cm}^{-1}\right) 3115$ (w), $2112(\mathrm{~s}), 1699$ (s), $1508(\mathrm{~m}), 1364(\mathrm{~s})$, $1342(\mathrm{~s}), 1193(\mathrm{~s}), 1167(\mathrm{~s}), 1143(\mathrm{~s}), 923(\mathrm{~m}), 831(\mathrm{~m}), 728(\mathrm{~m})$; HRMS (ESI) calcd. for $\mathrm{C}_{9} \mathrm{H}_{9} \mathrm{~N}_{2} \mathrm{O}_{2}{ }^{+}[\mathrm{M}+\mathrm{H}]^{+}$ 177.0659; found 177.0656. One carbon was not resolved at $100 \mathrm{MHz}$.

\footnotetext{
${ }^{2}$ S. P. Green, K. M. Wheelhouse, A. D. Payne, J. P. Hallett, P. W. Miller and J. A. Bull, Org. Process Res. Dev. 2020, 24,67 .

${ }^{3}$ G. Himbert, D. Fink and K. Diehl, Chem. Ber. 1988, 121, 431.
} 
<smiles>Cc1cc(C(C)(C)C)c(O)c(C(C)(C)C)c1</smiles>

20<smiles>CC1=CC(=O)OC(C)(C)O1</smiles>

21

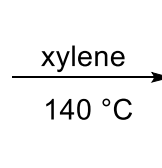<smiles>CC(=O)CC(=O)Oc1c(Br)cc(C)cc1C(C)(C)C</smiles>

22

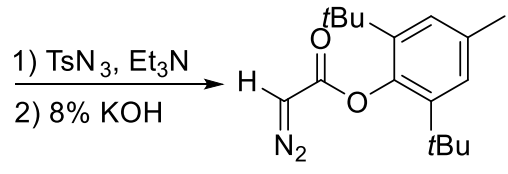

3d

Following a slightly modified procedure, ${ }^{4}$ a mixture of 2,6-di-tert-butyl-4-methylphenol (20) $(5.51 \mathrm{~g}$, $25.0 \mathrm{mmol}, 1.00$ equiv), 2,2,6-trimethyl-4H-1,3-dioxin-4-one (21) (3.32 mL, $25.0 \mathrm{mmol}, 1.00$ equiv), and xylene $\left(5 \mathrm{~mL}\right.$ ) was stirred at $140{ }^{\circ} \mathrm{C}$ for $1.5 \mathrm{~h}$. After cooling to room temperature, the reaction mixture was directly loaded on silica and was purified by column chromatography using EtOAc/pentane 2:98 as eluent to afford 2,6-di-tert-butyl-4-methylphenyl 3-oxobutanoate (22) as a white solid (5.77 g, 19.0 mmol, 76\%). ${ }^{1} \mathrm{H}$ NMR (400 MHz, $\left.\mathrm{CDCl}_{3}\right) \delta 12.08(\mathrm{~s}, 0.22 \mathrm{H}, \mathrm{OH}$ of enol form), $7.31-7.24(\mathrm{~m}, 1 \mathrm{H}, \mathrm{ArH}$ of enol and keto form), $7.24-7.18(\mathrm{~m}, 2 \mathrm{H}, \mathrm{ArH}$ of enol and keto form), $5.38(\mathrm{~s}, 0.2 \mathrm{H}$, vinyl $\mathrm{H}$ of enol form), $3.81\left(\mathrm{~s}, 1.56 \mathrm{H}, \mathrm{CH}_{3} \mathrm{COCH}_{2}\right.$ of keto form), $3.03\left(\mathrm{~m}, 2 \mathrm{H}, 2 \times \mathrm{CH}\left(\mathrm{CH}_{3}\right)_{2}\right.$ of enol and keto form), $2.41(\mathrm{~s}, 2.32 \mathrm{H}$, $\mathrm{CH}_{3} \mathrm{COCH}_{2}$ of keto form), 2.08 (s, $0.6 \mathrm{H}, \mathrm{CH}_{3}$ of enol form), $1.28-1.21\left(\mathrm{~m}, 12 \mathrm{H}, 2 \times \mathrm{CH}\left(\mathrm{CH}_{3}\right)_{2}\right) ;{ }^{13} \mathrm{C} \mathrm{NMR}$ (101 MHz, $\mathrm{CDCl}_{3}$ ), Enol form: $\delta 177.7,171.5,144.5,140.5,126.5,123.9,88.7,23.7,22.7,21.4 ;{ }^{13} \mathrm{C} \mathrm{NMR}$ (101 MHz, $\mathrm{CDCl}_{3}$ ), Keto form: $\delta 199.9,165.7,145.1,140.2,126.8,124.0,49.6,30.4,27.4,27.3$. The values of the NMR spectra are in accordance with reported literature data. ${ }^{5}$

Following a slightly modified procedure, ${ }^{4}$ to a solution of 2,6-di-tert-butyl-4-methylphenyl 3oxobutanoate (22) (5.48 g, $18.0 \mathrm{mmol}, 1.00$ equiv) in $\mathrm{MeCN}(22 \mathrm{~mL}$ ) was added triethylamine ( $3.26 \mathrm{~mL}$, $23.4 \mathrm{mmol}, 1.30$ equiv). The reaction mixture was cooled in an ice bath and a solution of tosyl azide ( $3.90 \mathrm{~g}, 19.8 \mathrm{mmol}, 1.1$ equiv) in $\mathrm{MeCN}(22 \mathrm{~mL}$ ) was added slowly. The reaction mixture was allowed to warm to room temperature and stirred for $20 \mathrm{~h}$. $8 \%$ aqueous $\mathrm{KOH}$ solution ( $90 \mathrm{~mL}$ ) was added and stirred vigorously for $4 \mathrm{~h}$. The reaction mixture was diluted with water $(50 \mathrm{~mL})$ and extracted with diethyl ether $(3 \times 100 \mathrm{~mL})$. The combined organic layers were dried over $\mathrm{MgSO}_{4}$, filtered and concentrated under reduced pressure. The crude product was purified by column chromatography using $\mathrm{Et}_{2} \mathrm{O} /$ pentane 2:98 as eluent to afford 2,6-di-tert-butyl-4-methylphenyl 2-diazoacetate (3d) as a yellow solid (4.80 g, $16.6 \mathrm{mmol}, 92 \%) .{ }^{1} \mathrm{H}$ NMR (400 MHz, CDCl $) \delta 7.12(\mathrm{~s}, 2 \mathrm{H}, \mathrm{ArH}), 5.00\left(\mathrm{~s}, 1 \mathrm{H}, \mathrm{CHN}_{2}\right.$ ), $2.32\left(\mathrm{~s}, 3 \mathrm{H}, \mathrm{ArCH}_{3}\right), 1.36(\mathrm{~s}, 18 \mathrm{H}, 2 \times t \mathrm{Bu}) ;{ }^{13} \mathrm{C} \mathrm{NMR}\left(101 \mathrm{MHz}, \mathrm{CDCl}_{3}\right) \delta 166.3,145.1,142.4,134.8,127.0$, $47.3,35.3,31.5,21.5$. The values of the NMR spectra are in accordance with reported literature data. ${ }^{6}$

\section{Furan-2-ylmethyl 2-diazoacetate (3e)}

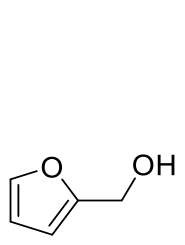

23

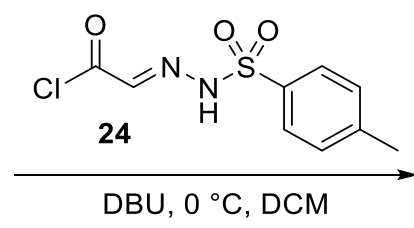

$\mathrm{DBU}, 0^{\circ} \mathrm{C}, \mathrm{DCM}$

Following a slightly modified procedure, ${ }^{7}$ to a solution of $p$-toluenesulfonylhydrazone of glyoxylic acid chloride (24) (1.30 g, $5.00 \mathrm{mmol}, 1.00$ equiv.) in $\mathrm{CH}_{2} \mathrm{Cl}_{2}(10 \mathrm{~mL}$ ) were added furfuryl alcohol (23) (475 $\mu \mathrm{L}, 5.50 \mathrm{mmol}, 1.10$ equiv.) and then $\mathrm{DBU}\left(1.89 \mathrm{~mL}, 12.5 \mathrm{mmol}, 2.50\right.$ equiv.) dropwise at $0{ }^{\circ} \mathrm{C}$. After

\footnotetext{
${ }^{4}$ P. Müller and P. Polleux, Helv. Chim. Acta 1994, 77, 645.

${ }^{5}$ D. P. Hari and J. Waser, J. Am. Chem. Soc., 2017, 139, 8420.

${ }^{6}$ M. P. Doyle, V. Bagheri, T. J. Wandless, N. K. Harn, D. A. Brinker, C. T. Eagle and K. L. Loh, J. Am. Chem. Soc. 1990, 112, 1906.

${ }^{7}$ T. Hashimoto, N. Uchiyama and K. Maruoka, J. Am. Chem. Soc. 2008, 130, 14380.
} 
stirring for $2 \mathrm{~h}$ at the same temperature, the reaction was stirred $30 \mathrm{~min}$ at room temperature and then poured into saturated $\mathrm{NH}_{4} \mathrm{Cl}$ solution $\left(10 \mathrm{~mL}\right.$ ). The organic layer was then extracted with $\mathrm{CH}_{2} \mathrm{Cl}_{2}$ $(3 \times 10 \mathrm{~mL})$, washed with brine $(20 \mathrm{~mL})$, dried over $\mathrm{MgSO}_{4}$, filtered and concentrated under reduced pressure. The crude product was purified by column chromatography using EtOAc/pentane 3:97 as eluent to afford furan-2-ylmethyl 2-diazoacetate (3e) as a yellow oil (534 mg, $3.21 \mathrm{mmol}, 64 \%$ ). ${ }^{1} \mathrm{H} \mathrm{NMR}$ $\left(400 \mathrm{MHz}, \mathrm{CDCl}_{3}\right) \delta 7.42$ (dd, $\left.J=1.9,0.9 \mathrm{~Hz}, 1 \mathrm{H}, \mathrm{ArH}\right), 6.42(\mathrm{dd}, J=3.3,0.8 \mathrm{~Hz}, 1 \mathrm{H}, \operatorname{ArH}), 6.36(\mathrm{dd}, J=$ 3.3, $1.8 \mathrm{~Hz}, 1 \mathrm{H}, \mathrm{ArH}), 5.14\left(\mathrm{~s}, 2 \mathrm{H}, \mathrm{CH}_{2} \mathrm{O}\right), 4.78\left(\mathrm{br} \mathrm{s}, 1 \mathrm{H}, \mathrm{CN}_{2} \mathrm{H}\right) ;{ }^{13} \mathrm{C} \mathrm{NMR}\left(101 \mathrm{MHz}, \mathrm{CDCl}_{3}\right) \delta$ 166.6, 149.5, $143.5,111.0,110.7,58.3,46.5$. The values of the NMR spectra are in accordance with reported literature data. ${ }^{8}$

\section{2-Diazoacetonitrile (3f)}

CAUTION: This diazo compound is reported to be explosive at high concentration.

$$
\underset{\mathrm{H}_{2} \mathrm{SO}_{4}}{\mathrm{H}_{2} \mathrm{~N}} \widehat{C N}
$$

25

$$
\underset{\mathrm{CH}_{2} \mathrm{Cl}_{2}}{\stackrel{\mathrm{NaNO}_{2}, \mathrm{H}_{2} \mathrm{O}}{\longrightarrow}}
$$<smiles>N#CC=N</smiles>

$3 \mathbf{f}$

Following a reported procedure, ${ }^{9}$ to a suspension of 2 -aminoacetonitrile sulfate (25) $(3.70 \mathrm{~g}, 24.0$ mmol, 1.00 equiv.) in $\mathrm{DCM}(28 \mathrm{~mL})$ at $-10^{\circ} \mathrm{C}$ was cautiously added dropwise an aqueous solution of sodium nitrite $(4.97 \mathrm{~g}, 72.0 \mathrm{mmol}, 3.00$ equiv.) in distilled water $(22 \mathrm{~mL})$ at such a rate that the temperature of the reaction did not rise above $0{ }^{\circ} \mathrm{C}$. During the addition effervescence was observed. After the complete addition, the reaction was allowed to stir for $30 \mathrm{~min}$ at $0{ }^{\circ} \mathrm{C}$. The organic layer was separated and the aqueous layer further extracted with DCM $(20 \mathrm{~mL})$. The combined organic layers were washed with $1 \%$ aqueous $\mathrm{NaHCO}_{3}$ solution $(10 \mathrm{~mL})$, dried over $\mathrm{MgSO}_{4}$, filtered and stored at - 18 ${ }^{\circ} \mathrm{C}$.

The concentration of the solution was assumed to be $0.5 \mathrm{M}$ of diazoacetonitrile (3f) in DCM and was used immediately without further purification.

\section{Diethyl (diazomethyl)phosphonate (3g)}<smiles>CCO[PH2+](=O)CC(C)=O</smiles>

26

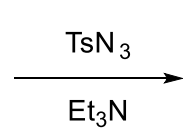<smiles>CCOC(=O)C(=O)OCC</smiles>

27

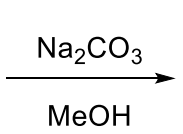<smiles>CCOC(=O)C#N</smiles>

$3 \mathbf{g}$

Following a reported procedure, ${ }^{10}$ a mixture of diethyl (2-oxopropyl)phosphonate (26) $(1.15 \mathrm{~mL}, 6.00$ mmol, 1.00 equiv), tosyl azide ( $1.3 \mathrm{~g}, 6.6 \mathrm{mmol}, 1.10$ equiv) and triethylamine $(6 \mathrm{~mL})$ was stirred at room temperature for $18 \mathrm{~h}$. After evaporation of the triethylamine under reduced pressure, the residue was dissolved in diethyl ether $(50 \mathrm{~mL})$. The precipitate was filtered off, the filtrate was evaporated and the residue was purified by column chromatography using EtOAc/pentane 50:50 as eluent affording the corresponding diethyl (1-diazo-2-oxopropyl)phosphonate (27) as a yellow oil (0.810 g, $3.68 \mathrm{mmol}, 61 \%) .{ }^{1} \mathrm{H} \mathrm{NMR}\left(400 \mathrm{MHz}, \mathrm{CDCl}_{3}\right) \delta 4.04-4.19\left(\mathrm{~m}, 4 \mathrm{H}, 2 \times \mathrm{CH}_{2} \mathrm{CH}_{3}\right) 2.19(\mathrm{~s}, 3 \mathrm{H}$, $\left.\mathrm{CH}_{3}\right), 1.30\left(\mathrm{t}, J=7.0 \mathrm{~Hz}, 6 \mathrm{H}, 2 \times \mathrm{CH}_{2} \mathrm{CH}_{3}\right) ;{ }^{13} \mathrm{C} \mathrm{NMR}\left(101 \mathrm{MHz}, \mathrm{CDCl}_{3}\right) \delta 190.1$ (d, $\left.J=13.2 \mathrm{~Hz}\right), 63.4(\mathrm{~d}, J=$ $5.6 \mathrm{~Hz}), 27.1,16.0(\mathrm{~d}, J=6.8 \mathrm{~Hz})$. The values of the NMR spectra are in accordance with reported literature data. ${ }^{5}$

\footnotetext{
${ }^{8}$ S. Bew, P.-A. Ashford and D. Bachera, Synthesis 2013, 45, 903.

${ }^{9}$ J. Dunn and A. P. Dobbs, Tetrahedron 2015, 71, 7386.

${ }^{10}$ S. Chanthamath, S. Ozaki, K. Shibatomi and S. Iwasa, Org. Lett. 2014, 16, 3012.
} 
To a solution of diethyl (1-diazo-2-oxopropyl)phosphonate (27) $(694 \mathrm{mg}, 3.15 \mathrm{mmol}, 1.00$ equiv) in $\mathrm{MeOH}\left(9.0 \mathrm{~mL}\right.$ ) was added $\mathrm{Na}_{2} \mathrm{CO}_{3}(401 \mathrm{mg}, 3.78 \mathrm{mmol}, 1.20$ equiv). The mixture was stirred at room temperature for $15 \mathrm{~min}$. The precipitate was filtered off, the filtrate was evaporated and the residue was purified by column chromatography using EtOAc/pentane $50: 50$ as eluent affording the corresponding diethyl (diazomethyl)phosphonate $(\mathbf{3 g})$ as a yellow oil $(533 \mathrm{mg}, 2.99 \mathrm{mmol}, 95 \%) .{ }^{1} \mathrm{H}$ $\operatorname{NMR}\left(400 \mathrm{MHz}, \mathrm{CDCl}_{3}\right) \delta 4.17-4.08\left(\mathrm{~m}, 4 \mathrm{H}, 2 \times \mathrm{CH}_{2} \mathrm{CH}_{3}\right), 3.75\left(\mathrm{~d}, J=11.1 \mathrm{~Hz}, 1 \mathrm{H}, \mathrm{CHN}_{2}\right), 1.34$ (td, $J=$ 7.1, $\left.0.7 \mathrm{~Hz}, 6 \mathrm{H}, 2 \times \mathrm{CH}_{2} \mathrm{CH}_{3}\right) ;{ }^{13} \mathrm{C} \mathrm{NMR}\left(101 \mathrm{MHz}, \mathrm{CDCl}_{3}\right) \delta 62.6(\mathrm{~d}, J=5.3 \mathrm{~Hz}), 16.1(\mathrm{~d}, J=6.9 \mathrm{~Hz})$. The values of the NMR spectra are in accordance with reported literature data. One carbon was not resolved at $101 \mathrm{MHz} .^{5}$

\section{Ethyl diazomethanesulfonate (3h)}

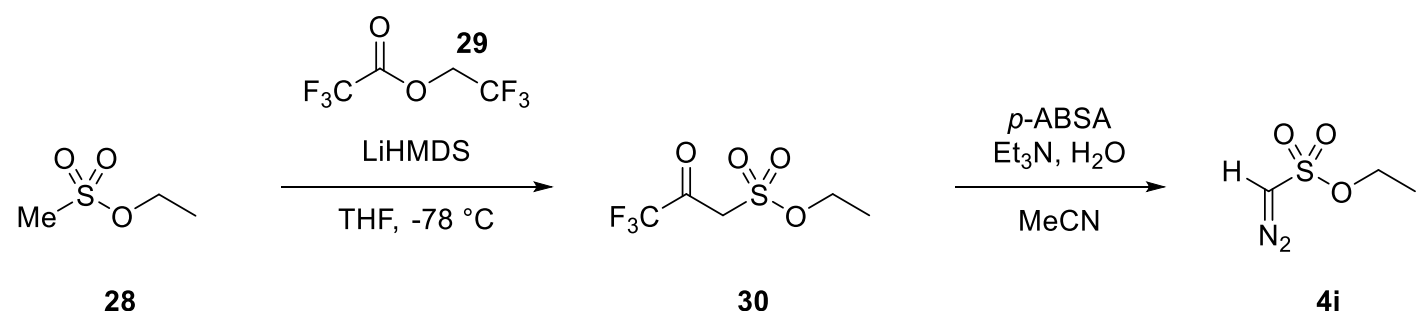

Following a reported procedure, ${ }^{11}$ to a solution of ethyl methanesulfonate (28) $(1.86 \mathrm{~g}, 15.0 \mathrm{mmol}$, 1.00 equiv) in dry THF ( $50 \mathrm{~mL}$ ) was added a $1 \mathrm{M}$ LiHMDS solution in hexane $(18 \mathrm{~mL}, 18 \mathrm{mmol}, 1.2$ equiv) at $-78{ }^{\circ} \mathrm{C}$. After stirring the reaction mixture for $30 \mathrm{~min}$ at this temperature, 2,2,2-trifluoroethyl trifluoroacetate (29) $(2.4 \mathrm{~mL}, 18 \mathrm{mmol}, 1.2$ equiv) was added rapidly in one portion via syringe. After $10 \mathrm{~min}$, the reaction mixture was poured into a solution of diethyl ether $(20 \mathrm{~mL})$ and $5 \% \mathrm{HCl}(50 \mathrm{~mL})$. The mixture was extracted with diethyl ether $(3 \times 50 \mathrm{~mL})$, washed with brine $(50 \mathrm{~mL})$, dried over $\mathrm{MgSO}_{4}$, filtered and concentrated under reduced pressure to give a yellow oil. The resulting ethyl 3,3,3trifluoro-2-oxopropane-1-sulfonate (30) was immediately dissolved in dry MeCN (30 mL). To this solution was added $p$-ABSA ( $4.32 \mathrm{~g}, 18.0 \mathrm{mmol}, 1.20$ equiv), $\mathrm{Et}_{3} \mathrm{~N}(2.5 \mathrm{~mL}, 18 \mathrm{mmol}, 1.2$ equiv), and water $(0.27 \mathrm{~mL}, 15 \mathrm{mmol}, 1.0$ equiv). After stirring the reaction mixture overnight at room temperature, the solvent was removed under reduced pressure and the residue was filtered on short plug of silica gel and washed with a mixture of ethyl acetate $(100 \mathrm{~mL})$ and hexane $(100 \mathrm{~mL})$. The filtrate was concentrated under vacuum and the residue was purified by column chromatography using EtOAc/pentane 10:90 as eluent to afford ethyl diazomethanesulfonate $(3 \mathrm{~h})$ as a yellow oil $(0.9 \mathrm{~g}, 6$ mmol, 40\%). ${ }^{1} \mathrm{H}$ NMR $\left(400 \mathrm{MHz}, \mathrm{CDCl}_{3}\right) \delta 5.25\left(\mathrm{~s}, 1 \mathrm{H}, \mathrm{CHN}_{2}\right), 4.26\left(\mathrm{q}, J=7.1 \mathrm{~Hz}, 2 \mathrm{H}, \mathrm{CH}_{2} \mathrm{CH}_{3}\right), 1.41(\mathrm{t}, J=$ $\left.7.1 \mathrm{~Hz}, 3 \mathrm{H}, \mathrm{CH}_{2} \mathrm{CH}_{3}\right) ;{ }^{13} \mathrm{C} \mathrm{NMR}\left(101 \mathrm{MHz}, \mathrm{CDCl}_{3}\right) \delta 67.4,52.4,14.6$. The values of the NMR spectra are in accordance with reported literature data. ${ }^{11}$

\section{2,2,2-Trifluorodiazoethane (3i)}

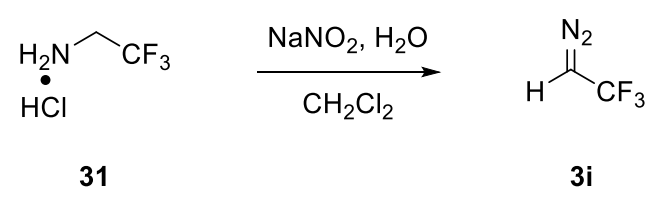

Following a reported procedure, ${ }^{12}$ under argon, 2,2,2-trifluoroethanamine hydrochloride (31) 0.678 $\mathrm{g}, 5.00 \mathrm{mmol}, 1.00$ equiv) and sodium nitrite $(0.379 \mathrm{~g}, 5.50 \mathrm{mmol}, 1.10$ equiv) were dissolved in degassed $\mathrm{CH}_{2} \mathrm{Cl}_{2}(10 \mathrm{~mL})$. Degassed water $\left(1.00 \mathrm{~mL}, 55.5 \mathrm{mmol}, 11.1\right.$ equiv) was added slowly at $0{ }^{\circ} \mathrm{C}$. The solution was stirred for $2 \mathrm{~h}$ at $0{ }^{\circ} \mathrm{C}$ and $1 \mathrm{~h}$ at room temperature. The aqueous layer was frozen in the freezer overnight $\left(-18^{\circ} \mathrm{C}\right)$ and the organic layer was dried over a plug of potassium carbonate, transferred into a vial, sealed and stored at $-18^{\circ} \mathrm{C}$. The concentration of the obtained solution was

\footnotetext{
${ }^{11}$ Ye T. and Zhou C., New J. Chem. 2005, 29, 1159.

${ }^{12}$ S. Hyde, J. Veliks, B. Liégault, D. Grassi, M. Taillefer and V. Gouverneur, Angew. Chem. Int. Ed., 2016, 55, 3785.
} 
determined to be $0.37 \mathrm{M}$ by ${ }^{19} \mathrm{~F}$ NMR analysis (according to an internal reference, $\mathrm{PhCF}_{3}$ ). ${ }^{19} \mathrm{~F} \mathrm{NMR} \mathrm{(377}$ $\left.\mathrm{MHz}, \mathrm{CH}_{2} \mathrm{Cl}_{2}\right) \delta$-55.56. The values of the NMR spectra are in accordance with reported literature data.

3-Diazo-1,1,1,2,2-pentafluoropropane (3j)

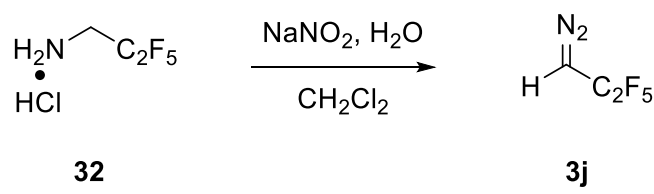

Under argon, 2,2,2-trifluoroethanamine hydrochloride (32) $(0.928 \mathrm{~g}, 5.00 \mathrm{mmol}, 1.00$ equiv) and sodium nitrite ( $0.379 \mathrm{~g}, 5.50 \mathrm{mmol}, 1.10$ equiv) were dissolved in degassed $\mathrm{CH}_{2} \mathrm{Cl}_{2}(10 \mathrm{~mL})$. Degassed water $\left(1.00 \mathrm{~mL}, 55.5 \mathrm{mmol}, 11.1\right.$ equiv) was added slowly at $0{ }^{\circ} \mathrm{C}$. The solution was stirred for $2 \mathrm{~h}$ at 0 ${ }^{\circ} \mathrm{C}$ and $1 \mathrm{~h}$ at room temperature. The organic layer was isolated, dried over $\mathrm{MgSO}_{4}$, transferred into a vial, sealed and stored at $-18{ }^{\circ} \mathrm{C}$. The concentration of the obtained solution was determined to be $0.36 \mathrm{M}$ by ${ }^{19} \mathrm{~F} \mathrm{NMR}$ analysis (according to an internal reference, $\left.\mathrm{PhCF}_{3}\right) \cdot{ }^{19} \mathrm{~F} \mathrm{NMR}\left(377 \mathrm{MHz}, \mathrm{CH}_{2} \mathrm{Cl}_{2}\right) \delta$ $88.96--89.01(m),-110.98--111.03(m)$.

\section{Ethyl 2-diazopropanoate (3k)}<smiles>CCOC(=O)C(C)C(C)=O</smiles>

33

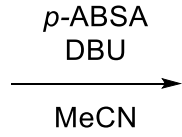

$\mathrm{MeCN}$<smiles>CCOC(=O)C(C)=N</smiles>

$3 k$

Following a modified reported procedure, ${ }^{13} \mathrm{DBU}(1.8 \mathrm{~mL}, 12 \mathrm{mmol}, 3.0$ equiv) was added slowly to a stirred solution of ethyl 2-methylacetoacetate (33) $(0.60 \mathrm{~mL}, 4.0 \mathrm{mmol}, 1.0$ equiv) and $p$-ABSA (1.4 g, $6.0 \mathrm{mmol}, 1.5$ equiv) in $\mathrm{MeCN}(80 \mathrm{~mL})$ at $0{ }^{\circ} \mathrm{C}$. The reaction mixture was then allowed to warm to room temperature. After stirring for $12 \mathrm{~h}$, the reaction mixture was quenched with $1 \mathrm{M} \mathrm{HCl}(8 \mathrm{~mL})$, and extracted with hexane $(3 \times 40 \mathrm{~mL})$. The organic layers were combined, washed with a saturated solution of $\mathrm{NaHCO}_{3}(40 \mathrm{~mL})$, brine $(40 \mathrm{~mL})$, dried over $\mathrm{MgSO}_{4}$, filtered and concentrated under reduced pressure. The crude product was purified by column chromatography using $\mathrm{Et}_{2} \mathrm{O}$ :pentane 2:98 as eluent affording the corresponding ethyl 2-diazopropanoate (3k) as a yellow oil (241 $\mathrm{mg}, 1.88 \mathrm{mmol}$, 47\%). ${ }^{1} \mathrm{H} \mathrm{NMR}\left(400 \mathrm{MHz}, \mathrm{CDCl}_{3}\right) \delta 4.20\left(\mathrm{q}, J=7.1 \mathrm{~Hz}, 2 \mathrm{H}, \mathrm{CH}_{2} \mathrm{CH}_{3}\right), 1.94\left(\mathrm{~s}, 3 \mathrm{H}, \mathrm{N}_{2} \mathrm{CCH}_{3}\right), 1.25$ (t, J = 7.1 $\left.\mathrm{Hz}, 3 \mathrm{H}, \mathrm{CH}_{2} \mathrm{CH}_{3}\right) ;{ }^{13} \mathrm{C} \mathrm{NMR}\left(101 \mathrm{MHz}, \mathrm{CDCl}_{3}\right) \delta 167.9,60.7,14.5,8.4$. The values of the NMR spectra are in accordance with reported literature data. ${ }^{14}$ One carbon was not resolved at $101 \mathrm{MHz}$.

\section{(1-Diazo-2,2,2-trifluoroethyl)benzene (3I)}<smiles>O=C(c1ccccc1)C(F)(F)F</smiles>

34<smiles>FC(F)(F)/N=C(/c1ccccc1)C(F)(F)F</smiles>

35

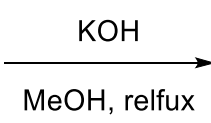<smiles>N#CC(F)(F)C(F)(F)c1ccccc1</smiles>

3!

2,2,2-Trifluoroacetophenone (34) (702 $\mu \mathrm{L}, 5.00 \mathrm{mmol}, 1.05$ equiv.) was added to EtOH (18.8 $\mathrm{mL}$ ) at room temperature followed by $p$-toluenesulfonylhydrazide ( $880 \mathrm{mg}, 4.76 \mathrm{mmol}, 1.00$ equiv.) in one portion. A condenser was then attached and the contents were heated to reflux for $12 \mathrm{~h}$. After this time, the solvent was removed under reduced pressure until the beginning of the formation of a solid.

\footnotetext{
${ }^{13}$ T. Hashimoto, Y. Naganawa and K. Maruoka, J. Am. Chem. Soc. 2011, 133, 8834

${ }^{14}$ L. Huang and W. D. Wulff, J. Am. Chem. Soc. 2011, 133, 8892.
} 
Then, pentane $(100 \mathrm{~mL})$ was added and the precipitate was collected by filtration and washed with pentane. The solid obtained was used in the next step without purification.

Following a reported procedure, $^{18}$ 4-methyl-N'-(2,2,2-trifluoro-1phenylethylidene)benzenesulfonohydrazide (35) was disolved in a $0.4 \mathrm{M}$ solution of potassium hydroxide $(561 \mathrm{mg}, 5.00 \mathrm{mmol}, 2.00$ equiv.) in $\mathrm{MeOH}(25.0 \mathrm{~mL})$. A condenser was attached and the reaction mixture was stirred at reflux for $0.5 \mathrm{~h}$. Then, the reaction was cooled to room temperature and diluted with water $(15 \mathrm{~mL})$. The crude product was extracted with pentane $(3 \times 20 \mathrm{~mL})$, dried over $\mathrm{MgSO}_{4}$, filtered and concentrated under reduced pressure. The crude oil was purified by silica gel chromatography using pentane as eluent to afford (1-diazo-2,2,2-trifluoroethyl)benzene (3I) as a volatile orange oil (344 mg, $1.85 \mathrm{mmol}, 37 \%)$. The compound was kept as a $0.6 \mathrm{M}$ solution in DCM at $18{ }^{\circ} \mathrm{C} .{ }^{1} \mathrm{H}$ NMR $\left(400 \mathrm{MHz}, \mathrm{CDCl}_{3}\right) \delta 7.45-7.36(\mathrm{~m}, 2 \mathrm{H}, \mathrm{ArH}), 7.23-7.17(\mathrm{~m}, 1 \mathrm{H}, \mathrm{ArH}), 7.13-7.05(\mathrm{~m}$, $2 \mathrm{H}, \mathrm{ArH}) ;{ }^{13} \mathrm{C}$ NMR $\left(101 \mathrm{MHz}, \mathrm{CDCl}_{3}\right) \delta 129.6,126.1,125.8$ (q, $\left.J=269.4 \mathrm{~Hz}\right), 123.7,122.4 ;{ }^{19} \mathrm{~F} \mathrm{NMR}(376$ $\left.\mathrm{MHz}, \mathrm{CDCl}_{3}\right) \delta-57.4$. One carbon was not resolved at $101 \mathrm{MHz}$. The values of the NMR spectra are in accordance with reported literature data. ${ }^{18}$

\section{3-Diazodihydrofuran-2(3H)-one (3m)}

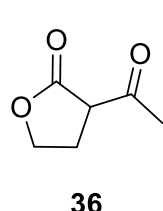

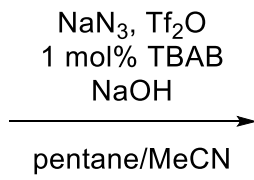

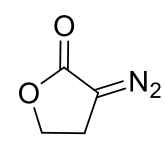

$3 m$

Following a reported procedure, ${ }^{15}$ sodium azide ( $2.42 \mathrm{~g}, 37.2 \mathrm{mmol}, 4.00$ equiv), sodium hydroxide (78 $\mathrm{mL}, 2 \mathrm{M}$ in water), tetrabutylammonium bromide ( $30.0 \mathrm{mg}, 93.0 \mu \mathrm{mol}, 0.01$ equiv), and pentane (40 $\mathrm{mL}$ ) were mixed in a $250 \mathrm{~mL}$ round-bottom flask with magnetic stirring bar open to the air and allowed to cool to $0^{\circ} \mathrm{C}$. With vigorous stirring, $\mathrm{Tf}_{2} \mathrm{O}(3.10 \mathrm{~mL}, 18.6 \mathrm{mmol}, 2.00$ equiv) was added dropwise. After $10 \mathrm{~min}$, a solution of 2-acetyl-butyrolactone (36) $(1.00 \mathrm{~mL}, 9.30 \mathrm{mmol}, 1.00$ equiv) in MeCN (35 mL) was poured into the round-bottom flask through a funnel, followed by additional MeCN $(10 \mathrm{~mL})$ to complete the transfer. The initially colorless reaction mixture immediately turned yellow. After allowing to stir for $30 \mathrm{~min}$ at $0{ }^{\circ} \mathrm{C}$, the mixture was diluted with ice water $(25 \mathrm{~mL})$ and EtOAc $(25 \mathrm{~mL})$ and transferred to a separatory funnel. After phase separation and removal of the organic layer, the aqueous layer was washed with cold EtOAc $(2 \times 50 \mathrm{~mL})$. The combined organic layers were dried over $\mathrm{MgSO}_{4}$, filtered, and concentrated under reduced pressure. The crude product was purified by column chromatography using EtOAc:pentane $50: 50$ as eluent affording the corresponding 3diazodihydrofuran-2(3H)-one ( $3 \mathrm{~m})$ as a bright yellow crystalline solid $(0.32 \mathrm{~g}, 2.8 \mathrm{mmol}, 30 \%) .{ }^{1} \mathrm{H}$ NMR $\left(400 \mathrm{MHz}, \mathrm{CDCl}_{3}\right) \delta 4.38\left(\mathrm{t}, J=8.0 \mathrm{~Hz}, 2 \mathrm{H}, \mathrm{CH}_{2}\right), 3.36\left(\mathrm{t}, J=8.0 \mathrm{~Hz}, 2 \mathrm{H}, \mathrm{CH}_{2}\right) ;{ }^{13} \mathrm{C} \mathrm{NMR}\left(101 \mathrm{MHz}, \mathrm{CDCl}_{3}\right)$ : 170.6, 65.3, 49.4, 23.1. The values of the NMR spectra are in accordance with reported literature data. ${ }^{15}$

\footnotetext{
${ }^{15}$ E. S. Sattely, S. J. Meek, S. J. Malcolmson, R. R. Schrock and A. H. Hoveyda, J. Am. Chem. Soc. 2009, 131, 943.
} 
Ethyl 2-diazo-6-hydroxyhexanoate (3n)

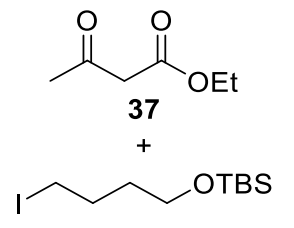

38
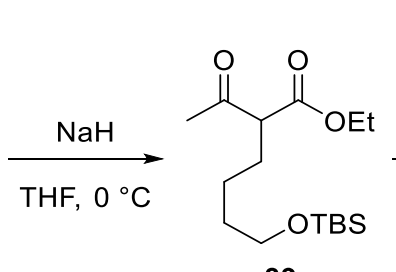

39

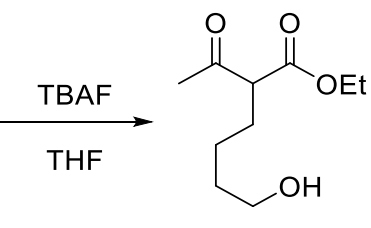

40

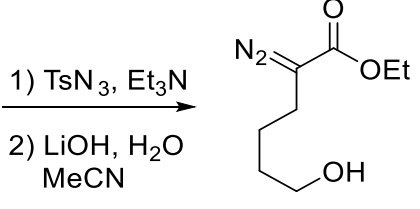

$3 n$

Adapted from a reported procedure, ${ }^{16}$ ethyl acetoacetate (37) $(3.79 \mathrm{~mL}, 30.0 \mathrm{mmol}, 2.00$ equiv.) was added dropwise to a stirred suspension of sodium hydride (60\% dispersion in mineral oil, $900 \mathrm{mg}, 22.5$ mmol, 1.50 equiv.) in dry THF $(35.7 \mathrm{~mL})$ at $0{ }^{\circ} \mathrm{C}$. After $30 \mathrm{~min}$, tert-butyl(4-iodobutoxy)dimethylsilane (38) (3.88 mL, $15.0 \mathrm{mmol}, 1.00$ equiv.) was added slowly at ambient temperature, and the reaction was refluxed for $24 \mathrm{~h}$. Saturated aqueous $\mathrm{NH}_{4} \mathrm{Cl}(50 \mathrm{~mL})$ was added, the two layers were separated, and the aqueous layer was extracted with diethyl ether $(3 \times 50 \mathrm{~mL})$. The combined organic layers were washed with brine, dried over $\mathrm{MgSO} 4$ and concentrated under reduced pressure. The residue was purified by column chromatography using EtOAc/pentane 5:95 as eluent to afford ethyl 2-acetyl-6((tert-butyldimethylsilyl)oxy)hexanoate (39) as a colorless oil $(3.66 \mathrm{~g}, 11.6 \mathrm{mmol}, 77 \%) . \mathrm{R}_{\mathrm{f}}=0.30$ (EtOAc/pentane 5:95); ${ }^{1} \mathrm{H} \mathrm{NMR}\left(400 \mathrm{MHz}, \mathrm{CDCl}_{3}\right) \delta 4.19$ (q, $\left.J=7.1 \mathrm{~Hz}, 2 \mathrm{H}, \mathrm{OCH}_{2} \mathrm{CH}_{3}\right), 3.59(\mathrm{t}, J=6.3 \mathrm{~Hz}$, $\left.2 \mathrm{H}, \mathrm{CH}_{2} \mathrm{OTBS}\right), 3.40(\mathrm{t}, J=7.4 \mathrm{~Hz}, 1 \mathrm{H}, \mathrm{C}(\mathrm{O}) \mathrm{CHC}(\mathrm{O})), 2.22\left(\mathrm{~s}, 3 \mathrm{H}, \mathrm{C}(\mathrm{O}) \mathrm{CH}_{3}\right), 1.95-1.76\left(\mathrm{~m}, 2 \mathrm{H}, \mathrm{CHCH}_{2}\right)$, 1.57-1.48 (m, 2H, CH $\left.\mathrm{CH}_{2} \mathrm{OTBS}\right), 1.38-1.23\left(\mathrm{~m}, 5 \mathrm{H}, \mathrm{OCH}_{2} \mathrm{CH}_{3}\right.$ and $\left.\mathrm{CH}_{2} \mathrm{CH}_{2} \mathrm{CH}\right), 0.88(\mathrm{~s}, 9 \mathrm{H}, \mathrm{Si}-\mathrm{tBu}), 0.03$ $\left(\mathrm{s}, 6 \mathrm{H}, 2 \times \mathrm{Si}-\mathrm{CH}_{3}\right) ;{ }^{13} \mathrm{C} \mathrm{NMR}\left(101 \mathrm{MHz}, \mathrm{CDCl}_{3}\right) \delta 203.4,170.0,62.9,61.5,60.1,32.6,28.9,28.2,26.1$, 24.0, 18.5, 14.3, -5.2; IR ( $\left.v_{\max }, \mathrm{cm}^{-1}\right) 2954(\mathrm{~m}), 2930(\mathrm{~m}), 2857(\mathrm{~m}), 1741(\mathrm{~m}), 1716(\mathrm{~s}), 1360(\mathrm{~m}), 1251$ $(\mathrm{m}), 1150(\mathrm{~m}), 1097(\mathrm{~s}), 835$ (s), 774 (s); HRMS (ESI/QTOF) m/z: [M+Na] ${ }^{+}$Calcd for $\mathrm{C}_{16} \mathrm{H}_{32} \mathrm{NaO}_{4} \mathrm{Si}^{+}$ 339.1962; Found 339.1961.

To a solution of ethyl 2-acetyl-6-((tert-butyldimethylsilyl)oxy)hexanoate (39) (3.17 g, $10.0 \mathrm{mmol}, 1.00$ equiv.) in THF ( $20 \mathrm{~mL}$ ) was added TBAF (11.0 mL, $11.0 \mathrm{mmol}, 1.10$ equiv., $1.0 \mathrm{M}$ in THF) slowly and the mixture was stirred overnight at room temperature. After this time, the reaction was quechend by a saturated aqueous $\mathrm{NH}_{4} \mathrm{Cl}$ solution $(20 \mathrm{~mL})$ and diluted with diethyl ether $(20 \mathrm{~mL})$. The aqueous layer was extracted with diethyl ether $(3 \times 10 \mathrm{~mL})$. The combined organic layers were washed with brine (20 $\mathrm{mL}$ ), dried over $\mathrm{MgSO}_{4}$, filtered and concentrated under reduced pressure. The crude oil was purified by flash column chromatography using EtOAc/pentane 50:50 as eluent to afford ethyl 2-acetyl-6hydroxyhexanoate (40) as a colorless oil $(1.51 \mathrm{~g}, 7.49 \mathrm{mmol}, 75 \%) . \mathrm{R}_{\mathrm{f}}=0.35$ (EtOAc/pentane 50:50); ${ }^{1} \mathrm{H}$ $\operatorname{NMR}\left(400 \mathrm{MHz}, \mathrm{CDCl}_{3}\right) \delta 4.20\left(\mathrm{qd}, J=7.1,0.9 \mathrm{~Hz}, 2 \mathrm{H}, \mathrm{OCH}_{2} \mathrm{CH}_{3}\right), 3.64\left(\mathrm{t}, J=6.4 \mathrm{~Hz}, 2 \mathrm{H}, \mathrm{CH}_{2} \mathrm{OH}\right), 3.42(\mathrm{t}$, $J=7.3 \mathrm{~Hz}, 1 \mathrm{H}, \mathrm{C}(\mathrm{O}) \mathrm{CHC}(\mathrm{O})), 2.23\left(\mathrm{~s}, 3 \mathrm{H}, \mathrm{C}(\mathrm{O}) \mathrm{CH}_{3}\right), 1.93-1.80\left(\mathrm{~m}, 2 \mathrm{H}, \mathrm{CHCH}_{2}\right), 1.64-1.53(\mathrm{~m}, 3 \mathrm{H}$, $\mathrm{CH}_{2} \mathrm{CH}_{2} \mathrm{OH}$ and $\left.\mathrm{OH}\right), 1.42-1.31\left(\mathrm{~m}, 2 \mathrm{H}, \mathrm{CH}_{2} \mathrm{CH}_{2} \mathrm{CH}\right), 1.27\left(\mathrm{t}, J=7.1 \mathrm{~Hz}, 3 \mathrm{H}, \mathrm{OCH}_{2} \mathrm{CH}_{3}\right) ;{ }^{13} \mathrm{C}$ NMR $(101$ $\left.\mathrm{MHz}, \mathrm{CDCl}_{3}\right) \delta 203.3,170.0,62.5,61.5,59.9,32.4,29.0,27.9,23.8,14.3 ; \mathrm{IR}\left(\mathrm{v}_{\max }, \mathrm{cm}^{-1}\right) 3436(\mathrm{w}), 2938$ $(\mathrm{m}), 2869(\mathrm{w}), 1736(\mathrm{~s}), 1711(\mathrm{~s}), 1361(\mathrm{~m}), 1201(\mathrm{~s}), 1149(\mathrm{~s}), 1056(\mathrm{~m}), 1032(\mathrm{~s})$; HRMS (ESI/QTOF) $\mathrm{m} / \mathrm{z}$ : [M+Na] ${ }^{+}$Calcd for $\mathrm{C}_{10} \mathrm{H}_{18} \mathrm{NaO}_{4}{ }^{+}$225.1097; Found 225.1101.

Adapted from a reported procedure, ${ }^{16}$ a $1.0 \mathrm{M}$ solution of tosylazide $(2.07 \mathrm{~g}, 10.5 \mathrm{mmol}, 1.50$ equiv.) in $\mathrm{MeCN}(10.5 \mathrm{~mL})$ was added dropwise to a solution of 2-acetyl-6-hydroxyhexanoate (40) $(1.46 \mathrm{~g}, 7.00$ mmol, 1.00 equiv.) and triethylamine $(1.46 \mathrm{~mL}, 10.5 \mathrm{mmol}, 1.50$ equiv.) in $\mathrm{MeCN}(21.2 \mathrm{~mL})$ at ambient temperature. After stirring for $12 \mathrm{~h}$, a solution of $\mathrm{LiOH}(0.84 \mathrm{~g}, 35 \mathrm{mmol}, 5.0$ equiv.) in water $(12.7 \mathrm{~mL})$ was added and the mixture was stirred for another $12 \mathrm{~h}$. Brine was added, the two layers were separated, and the aqueous layer was extracted with $\mathrm{Et}_{2} \mathrm{O}(3 \times 30 \mathrm{~mL})$. The combined organic layers were washed with brine, dried over $\mathrm{MgSO}_{4}$ and concentrated under reduced pressure. The residue was purified by flash column chromatography using EtOAc/pentane 25:75 as eluent to afford ethyl 2diazo-6-hydroxyhexanoate ( $3 \mathrm{n}$ ) as a bright yellow oil $(0.95 \mathrm{~g}, 5.1 \mathrm{mmol}, 73 \%) . \mathrm{R}_{\mathrm{f}}=0.31$ (EtOAc/pentane 25:75); ${ }^{1} \mathrm{H} \mathrm{NMR}\left(400 \mathrm{MHz}, \mathrm{CDCl}_{3}\right) \delta 4.21$ (q, $\left.J=7.1 \mathrm{~Hz}, 2 \mathrm{H}, \mathrm{OCH}_{2} \mathrm{CH}_{3}\right), 3.77-3.61\left(\mathrm{~m}, 2 \mathrm{H}, \mathrm{CH}_{2} \mathrm{OH}\right), 2.43$

\footnotetext{
${ }^{16}$ S.-F. Zhu, X.-G. Song, Y. Li, Y. Cai and Q.-L. Zhou, J. Am. Chem. Soc. 2010, 132, 16374.
} 
$-2.30\left(\mathrm{~m}, 2 \mathrm{H}, \mathrm{CH}_{2} \mathrm{CN}_{2}\right), 1.69-1.54\left(\mathrm{~m}, 4 \mathrm{H}, \mathrm{CH}_{2} \mathrm{CH}_{2}\right), 1.51$ (br s, $\left.1 \mathrm{H}, \mathrm{OH}\right), 1.27\left(\mathrm{t}, J=7.1 \mathrm{~Hz}, 3 \mathrm{H}, \mathrm{OCH}_{2} \mathrm{CH}_{3}\right)$; ${ }^{13} \mathrm{C} \mathrm{NMR}\left(101 \mathrm{MHz}, \mathrm{CDCl}_{3}\right) \delta 167.8,62.5,60.9,31.7,24.2,23.1,14.7 ; \mathrm{IR}\left(\mathrm{v}_{\max }, \mathrm{cm}^{-1}\right) 3437(\mathrm{w}), 2939(\mathrm{w})$, $2868(\mathrm{w}), 2079$ (s), $1686(\mathrm{~s}), 1371(\mathrm{~s}), 1328(\mathrm{~m}), 1305$ (s), 1276 (m), 1171 (s), 1119 (s), 1057 (m), 1024 (m), 740 (s); HRMS (APPI/LTQ-Orbitrap) m/z: [M-N $]^{+}$Calcd. for $\mathrm{C}_{8} \mathrm{H}_{14} \mathrm{O}_{3}{ }^{+}$158.0937; Found 158.0937. One carbon was not resolved at $101 \mathrm{MHz}$.

\section{1,4-Dichloro-2-(1-diazo-2,2,2-trifluoroethyl)benzene (30)}

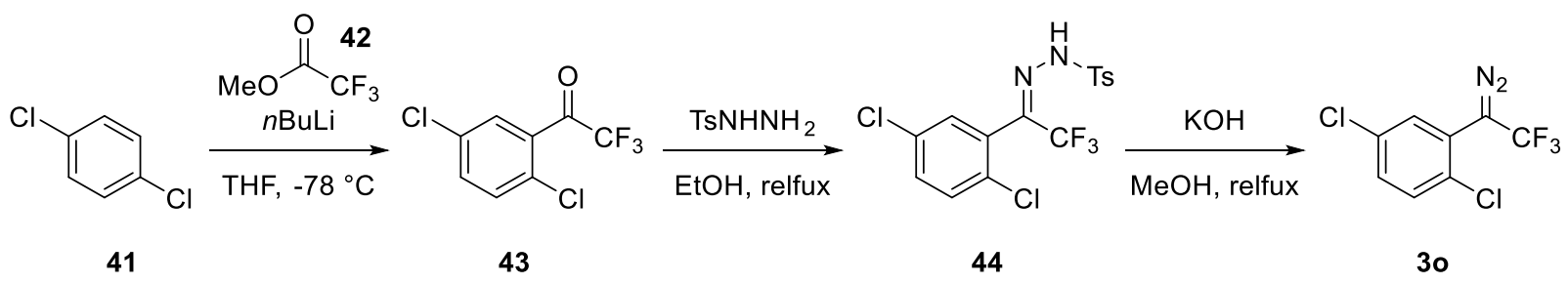

Following a modified reported procedure, ${ }^{17}$ a solution of 1,4-dichlorobenzene (41) (10.0 g, $68.0 \mathrm{mmol}$, 1.00 equiv.) in anhydrous THF ( $150 \mathrm{~mL}$ ) was cooled to $-78^{\circ} \mathrm{C}$. Then, a $2.5 \mathrm{M}$ solution of $n$-butyllithium ( $30.0 \mathrm{~mL}, 74.8 \mathrm{mmol}, 1.10$ equiv.) in hexanes was added dropwise. The mixture was stirred for $1 \mathrm{~h}$, followed by the dropwise addition of methyl 2,2,2-trifluoroacetate (42) $(7.66 \mathrm{~mL}, 76.0 \mathrm{mmol}, 1.12$ equiv.) in $30 \mathrm{~min}$. The mixture was allowed to warm up to $0{ }^{\circ} \mathrm{C}$, stirred for $2 \mathrm{~h}$ and then quenched with saturated aqueous ammonium chloride solution $(50 \mathrm{~mL})$. Diethyl ether $(50 \mathrm{~mL})$ was added and the layers were separated. The aqueous layer was extracted with diethyl ether $(50 \mathrm{~mL})$. The combined organic layers were washed with brine $(50 \mathrm{~mL})$, dried over $\mathrm{MgSO}_{4}$, filtrated and concentrated under reduced pressure. The residue was purified by Kugelrohr distillation to afford 1-(2,5-dichloro-phenyl)2,2,2-trifluoroethanone (43) as a colorless oil (12.2 g, $\left.50.4 \mathrm{mmol}, 74 \%) .{ }^{1} \mathrm{H} \mathrm{NMR} \mathrm{(400} \mathrm{MHz}, \mathrm{CDCl}_{3}\right): \delta$ $7.67(\mathrm{~s}, 1 \mathrm{H}, \mathrm{ArH}), 7.49-7.54(\mathrm{~m}, 2 \mathrm{H}, \mathrm{ArH}) ;{ }^{13} \mathrm{C} \mathrm{NMR}\left(101 \mathrm{MHz}, \mathrm{CDCl}_{3}\right): \delta 180.8(\mathrm{q}, J=37.6 \mathrm{~Hz}), 134.0$, $133.1,132.7,132.1,131.8,129.6$ (q, $J=2.2 \mathrm{~Hz}), 115.5$ (q, $J=287.3 \mathrm{~Hz}) ;{ }^{19} \mathrm{~F} \mathrm{NMR}\left(376 \mathrm{MHz}, \mathrm{CDCl}_{3}\right) \delta-$ 73.4. The values of the NMR spectra are in accordance with reported literature data. ${ }^{17}$

Adapted from a reported procedure, ${ }^{18}$ 1-(2,5-dichlorophenyl)-2,2,2-trifluoroethanone (43) (7.29 g, $30.0 \mathrm{mmol}, 1.05$ equiv.) was added to $\mathrm{EtOH}(19 \mathrm{~mL})$ at room temperature followed by $p$ toluenesulfonylhydrazide $(5.32 \mathrm{~g}, 28.6 \mathrm{mmol}, 1.00$ equiv.) in one portion. A condenser was then attached and the contents were heated to reflux for $12 \mathrm{~h}$. After this time, the solvent was removed under reduced pressure until the beginning of the formation of a solid. Then, pentane $(200 \mathrm{~mL})$ was added and the precipitate was collected by filtration and washed with pentane. The solid obtained was used in the next step without purification.

Adapted from a reported procedure, ${ }^{18} \quad N^{\prime}$-(1-(2,5-dichlorophenyl)-2,2,2-trifluoroethylidene)-4methylbenzenesulfonohydrazide (44) was disolved in a $0.4 \mathrm{M}$ solution of potassium hydroxide ( $3.37 \mathrm{~g}$, $60.0 \mathrm{mmol}, 2.00$ equiv.) in $\mathrm{MeOH}(17.5 \mathrm{~mL})$. A condenser was attached and the reaction mixture was stirred at reflux for $0.5 \mathrm{~h}$. Then, the reaction was cooled to room temperature and diluted with water $(20 \mathrm{~mL})$. The product was extracted with $\mathrm{Et}_{2} \mathrm{O}(3 \times 30 \mathrm{~mL})$, dried over $\mathrm{MgSO}_{4}$, filtered and concentrated under reduced pressure. The crude oil was purified by silica gel chromatography using pentane as eluent to afford 1,4-dichloro-2-(1-diazo-2,2,2-trifluoroethyl)benzene (3o) as a orange oil (1.69 g, 6.09 $\mathrm{mmol}, 20 \%$ ). The compound was kept as a $0.6 \mathrm{M}$ solution in $\mathrm{DCM}$ at $-18{ }^{\circ} \mathrm{C} . \mathrm{R}_{\mathrm{f}}=0.95$ (pentane); ${ }^{1} \mathrm{H}$ NMR $\left(400 \mathrm{MHz}, \mathrm{CDCl}_{3}\right) \delta 7.43-7.36(\mathrm{~m}, 2 \mathrm{H}, \mathrm{ArH}), 7.30-7.25(\mathrm{~m}, 1 \mathrm{H}, \mathrm{ArH}) ;{ }^{13} \mathrm{C} \mathrm{NMR}\left(101 \mathrm{MHz}, \mathrm{CDCl}_{3}\right) \delta$ 133.7, 132.0, 131.8, 129.8, 129.5, 125.7 (q, $J=269.5 \mathrm{~Hz}), 123.8 ;{ }^{19} \mathrm{~F} \mathrm{NMR}\left(376 \mathrm{MHz}, \mathrm{CDCl}_{3}\right) \delta-56.8$; IR $\left(v_{\text {max }}, \mathrm{cm}^{-1}\right) 2096(\mathrm{~s}), 1583(\mathrm{~m}), 1470(\mathrm{~m}), 1320(\mathrm{~s}), 1251(\mathrm{~m}), 1176(\mathrm{~s}), 1149(\mathrm{~s}), 1107(\mathrm{~s}), 1060(\mathrm{~m}), 977$ (s), 815 (m), 795 (m), 729 (m); HRMS (APPI/LTQ-Orbitrap) m/z: [M] ${ }^{+}$Calcd. for $\mathrm{C}_{8} \mathrm{H}_{3} \mathrm{Cl}_{2} \mathrm{~F}_{3}{ }^{+}$225.9558; Found 225.9565. One carbon was not resolved at $101 \mathrm{MHz}$.

\footnotetext{
${ }^{17}$ A. S. Golubev, A. F. Shidlovskii, A. S. Peregudov and N. D. Kagramanov, Russ Chem Bull. 2014, 63, 2264.
} 
<smiles>CCO[14CH2]N</smiles>

45<smiles>FC(F)(F)N/N=C(/c1cccc(C(F)(F)F)c1)C(F)(F)F</smiles>

46

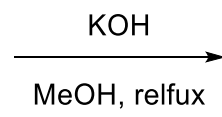<smiles>N#CC(F)(F)C(F)(F)c1cccc(C(F)(F)F)c1</smiles>

$3 p$

2,2,2-Trifluoro-1-(3-(trifluoromethyl)phenyl)ethanone (45) $(0.605 \mathrm{~g}, 2.50 \mathrm{mmol}, 1.05$ equiv.) was added to EtOH $(4.7 \mathrm{~mL})$ at room temperature followed by $p$-toluenesulfonylhydrazide $(0.443 \mathrm{~g}, 2.38$ mmol, 1.00 equiv.) in one portion. A condenser was then attached and the contents were heated to reflux for $12 \mathrm{~h}$. After this time, the solvent was removed under reduced pressure until the beginning of the formation of a solid. Then, pentane $(100 \mathrm{~mL})$ was added and the precipitate was collected by filtration and washed with pentane. The solid obtained was used in the next step without purification.

Adapted from a reported procedure, ${ }^{18}$ 4-methyl-N'-(2,2,2-trifluoro-1(3(trifluoromethyl)phenyl)ethylidene)benzenesulfonohydrazide (46) was disolved in a $0.4 \mathrm{M}$ solution of potassium hydroxide ( $281 \mathrm{mg}, 5.00 \mathrm{mmol}, 2.00$ equiv.) in $\mathrm{MeOH}(6.25 \mathrm{~mL}$ ). A condenser was attached and the reaction mixture was stirred at reflux for $0.5 \mathrm{~h}$. Then, the reaction was cooled to room temperature and diluted with water $(15 \mathrm{~mL})$. The crude product was extracted with pentane ( 3 $\times 20 \mathrm{~mL}$ ), dried over $\mathrm{MgSO}_{4}$, filtered and concentrated under reduced pressure. The crude oil was purified by silica gel chromatography using EtOAc/pentane 1:99 as eluent to afford 1-(1-diazo-2,2,2trifluoroethyl)-3-(trifluoromethyl)benzene $(3 \mathrm{p})$ as a volatile orange oil $(233 \mathrm{mg}, 0.834 \mathrm{mmol}, 33 \%$, contains 10 wt. \% of eluent). The compound was kept as a $0.6 \mathrm{M}$ solution in DCM at $-18{ }^{\circ} \mathrm{C} . \mathrm{R}_{\mathrm{f}}=0.95$ (EtOAc/pentane 1:99); ${ }^{1} \mathrm{H}$ NMR $\left(400 \mathrm{MHz}, \mathrm{CDCl}_{3}\right) \delta 7.53(\mathrm{tt}, \mathrm{J}=7.8,0.8 \mathrm{~Hz}, 1 \mathrm{H}, \mathrm{ArH}), 7.49-7.41(\mathrm{~m}, 1 \mathrm{H}$, $\operatorname{ArH}), 7.33-7.23(\mathrm{~m}, 2 \mathrm{H}, \mathrm{ArH}) ;{ }^{13} \mathrm{C} \mathrm{NMR}\left(101 \mathrm{MHz}, \mathrm{CDCl}_{3}\right) \delta 132.2(\mathrm{q}, J=32.6 \mathrm{~Hz}), 130.1,125.3,125.3$ $(q, J=269.6 \mathrm{~Hz}), 125.2,123.8(\mathrm{q}, J=272.5 \mathrm{~Hz}), 122.7(\mathrm{q}, J=3.8 \mathrm{~Hz}), 118.9-118.6(\mathrm{~m}) ;{ }^{19} \mathrm{~F} \mathrm{NMR}(376$ $\left.\mathrm{MHz}, \mathrm{CDCl}_{3}\right) \delta$-57.4, -63.1; IR ( $\left.\mathrm{v}_{\max }, \mathrm{cm}^{-1}\right) 2089(\mathrm{~s}), 1616(\mathrm{w}), 1591(\mathrm{w}), 1496(\mathrm{w}), 1455(\mathrm{~m}), 1362(\mathrm{~m})$, 1338 (s), 1312 (s), 1273 (m), 1168 (s), 1111 (s), 1076 (s), 977 (m), 795 (s), 692 (s); HRMS (ESI/QTOF) $\mathrm{m} / \mathrm{z}$ : $\left[\mathrm{M}-\mathrm{N}_{2}+\mathrm{H}\right]^{+}$Calcd. for $\mathrm{C}_{9} \mathrm{H}_{5} \mathrm{~F}_{6}{ }^{+}$227.0290; Found 227.0291. One carbon was not resolved at $101 \mathrm{MHz}$.

\section{4-(1-Diazo-2,2,2-trifluoroethyl)-1,1'-biphenyl (3q)}

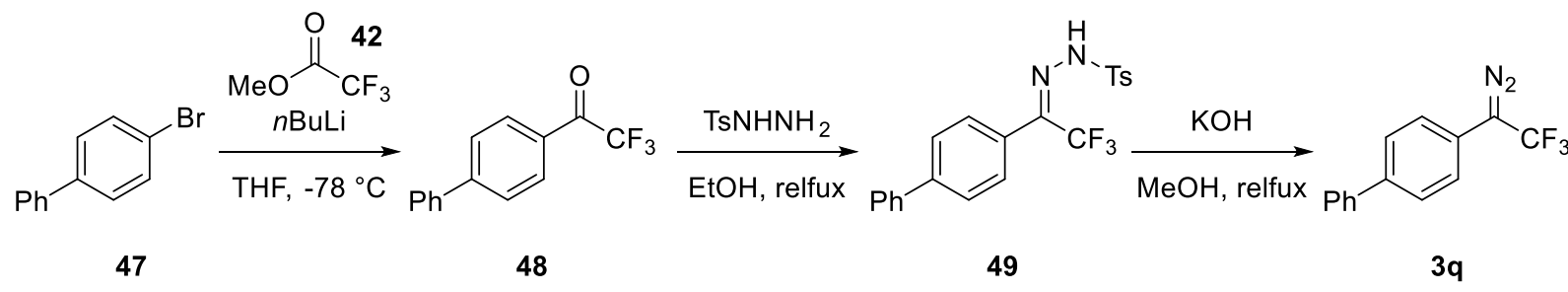

A solution of 4-bromo-biphenyl (47) $(4.66 \mathrm{~g}, 20.0 \mathrm{mmol}, 1.00$ equiv.) in anhydrous THF (100 mL) was cooled to $-78{ }^{\circ} \mathrm{C}$. Then, a $2.5 \mathrm{M}$ solution of $n$-butyllithium $(9.60 \mathrm{~mL}, 24.0 \mathrm{mmol}, 1.20$ equiv.) in hexanes was added dropwise. The mixture was stirred for $1 \mathrm{~h}$, followed by the dropwise addition of methyl 2,2,2-trifluoroacetate (42) $(2.21 \mathrm{~mL}, 22.0 \mathrm{mmol}, 1.10$ equiv.) in $30 \mathrm{~min}$. The mixture was allowed to warm up to room temperature, stirred for $18 \mathrm{~h}$ and then quenched with saturated aqueous ammonium chloride solution $(50 \mathrm{~mL})$. Diethyl ether $(50 \mathrm{~mL})$ was added and the layers were separated. The aqueous layer was extracted with diethyl ether $(2 \times 25 \mathrm{~mL})$. The combined organic layers were washed with brine $(50 \mathrm{~mL})$, dried over $\mathrm{MgSO}_{4}$, filtrated and concentrated under reduced pressure. The residue was

\footnotetext{
${ }^{18}$ E. Emer, J. Twilton, M. Tredwell, S. Calderwood, T. L. Collier, B. Liégault, M. Taillefer and V. Gouverneur, Org. Lett. 2014, 16, 6004.
} 
purified by silica gel chromatography using pentane/EtOAc $90: 10$ as eluent to afford 1-([1,1'-biphenyl]4-yl)-2,2,2-trifluoroethanone (48) as a slight yellow oil (3.37 g, $13.5 \mathrm{mmol}, 67 \%) .{ }^{1} \mathrm{H}$ NMR $(400 \mathrm{MHz}$, $\left.\mathrm{CDCl}_{3}\right) \delta 8.20-8.10(\mathrm{~m}, 2 \mathrm{H}, \mathrm{ArH}), 7.81-7.74(\mathrm{~m}, 2 \mathrm{H}, \mathrm{ArH}), 7.68-7.62(\mathrm{~m}, 2 \mathrm{H}, \mathrm{ArH}), 7.54-7.41(\mathrm{~m}$, $3 \mathrm{H}, \mathrm{ArH}) ;{ }^{13} \mathrm{C}$ NMR (101 MHz, CDCl $) \delta 180.3$ (q, J=34.8 Hz), 148.4, 139.3, 130.9 (q, $\left.J=2.2 \mathrm{~Hz}\right), 129.3$, $129.1,128.7,127.8,127.5,116.9(\mathrm{q}, J=291.4 \mathrm{~Hz}) ;{ }^{19} \mathrm{~F} \mathrm{NMR}\left(376 \mathrm{MHz}, \mathrm{CDCl}_{3}\right) \delta-71.32$. The values of the NMR spectra are in accordance with reported literature data. ${ }^{18}$

Following a reported procedure, ${ }^{18} 1$-([1,1'-biphenyl]-4-yl)-2,2,2-trifluoroethanone (48) (3.36 g, 13.5 mmol, 1.05 equiv.) was added to $\mathrm{EtOH}(9 \mathrm{~mL})$ at room temperature followed by $p$ toluenesulfonylhydrazide $(2.40 \mathrm{~g}, 12.9 \mathrm{mmol}, 1.00$ equiv.) in one portion. A condenser was then attached and the contents were heated to reflux for $12 \mathrm{~h}$. After this time, the solvent was removed under reduced pressure until the beginning of the formation of a solid. Then, pentane $(200 \mathrm{~mL})$ was added and the precipitate was collected by filtration and washed with pentane. The solid obtained was used in the next step without purification.

Following a reported procedure, ${ }^{18} \quad N^{\prime}$-(1-([1,1'-biphenyl]-4-yl)-2,2,2-trifluoroethylidene)-4methylbenzenesulfonohydrazide (49) was disolved in a $0.4 \mathrm{M}$ solution of potassium hydroxide ( $3.37 \mathrm{~g}$, $60.0 \mathrm{mmol}, 2.00$ equiv.) in $\mathrm{MeOH}(17.5 \mathrm{~mL})$. A condenser was attached and the reaction mixture was stirred at reflux for $0.5 \mathrm{~h}$. Then, the reaction was cooled to room temperature and diluted with water $(20 \mathrm{~mL})$. The product was extracted with $\mathrm{Et}_{2} \mathrm{O}(3 \times 30 \mathrm{~mL})$, dried over $\mathrm{MgSO}_{4}$, filtered and concentrated under reduced pressure. The crude oil was purified by silica gel chromatography using pentane as eluent to afford 4-(1-Diazo-2,2,2-trifluoroethyl)-1,1'-biphenyl (3q) as a red solid (1.42 g, $5.44 \mathrm{mmol}$, $50 \%)$. The compound was kept at $-18{ }^{\circ} \mathrm{C} . \mathrm{R}_{\mathrm{f}}=0.70$ (pentane); ${ }^{1} \mathrm{H}$ NMR $\left(400 \mathrm{MHz}, \mathrm{CDCl}_{3}\right) \delta 7.68-7.62$ $(\mathrm{m}, 2 \mathrm{H}, \mathrm{ArH}), 7.62-7.55(\mathrm{~m}, 2 \mathrm{H}, \mathrm{ArH}), 7.45(\mathrm{dd}, J=8.4,6.9 \mathrm{~Hz}, 2 \mathrm{H}, \mathrm{ArH}), 7.41-7.34(\mathrm{~m}, 1 \mathrm{H}, \mathrm{ArH}), 7.17$ $(\mathrm{d}, J=8.2 \mathrm{~Hz}, 2 \mathrm{H}, \mathrm{ArH}) ;{ }^{13} \mathrm{C}$ NMR $\left(101 \mathrm{MHz}, \mathrm{CDCl}_{3}\right) \delta 140.2,139.0,129.1,128.2,127.7,127.0,125.8$ (q, $J=269.6 \mathrm{~Hz}), 122.7,122.4 ;{ }^{19} \mathrm{~F} \mathrm{NMR}\left(376 \mathrm{MHz}, \mathrm{CDCl}_{3}\right) \delta-57.32$. One carbon was not resolved at 101 $\mathrm{MHz}$. The values of the NMR spectra are in accordance with reported literature data. ${ }^{18}$

\section{1-Bromo-4-(1-diazo-2,2,2-trifluoroethyl)benzene (3r)}<smiles>O=C(c1ccc(Br)cc1)C(F)(F)F</smiles>

50

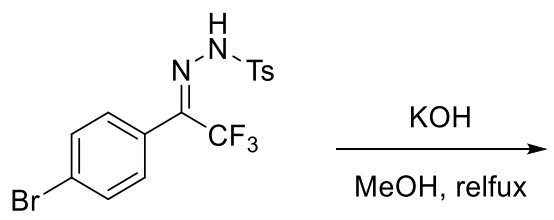

51<smiles>N#CC(F)(F)c1ccc(Br)cc1</smiles>

$3 r$

1-(4-Bromophenyl)-2,2,2-trifluoroethanone (50) $(633 \mathrm{mg}, 2.50 \mathrm{mmol}, 1.05$ equiv.) was added to EtOH $(4.7 \mathrm{~mL})$ at room temperature followed by $p$-toluenesulfonylhydrazide $(0.443 \mathrm{~g}, 2.38 \mathrm{mmol}, 1.00$ equiv.) in one portion. A condenser was then attached and the contents were heated to reflux for 12 h. After this time, the solvent was removed under reduced pressure until the beginning of the formation of a solid. Then, pentane $(100 \mathrm{~mL})$ was added and the precipitate was collected by filtration and washed with pentane. The solid obtained was used in the next step without purification.

Following a reported procedure, ${ }^{18} \quad \mathrm{~N}^{\prime}$-(1-(4-bromophenyl)-2,2,2-trifluoroethylidene)-4methylbenzenesulfonohydrazide (51) was disolved in a $0.4 \mathrm{M}$ solution of potassium hydroxide (281 $\mathrm{mg}, 5.00 \mathrm{mmol}, 2.00$ equiv.) in $\mathrm{MeOH}(12.5 \mathrm{~mL})$. A condenser was attached and the reaction mixture was stirred at reflux for $0.5 \mathrm{~h}$. Then, the reaction was cooled to room temperature and diluted with water $(15 \mathrm{~mL})$. The crude product was extracted with pentane $(3 \times 20 \mathrm{~mL})$, dried over $\mathrm{MgSO}_{4}$, filtered and concentrated under reduced pressure. The crude oil was purified by silica gel chromatography using pentane as eluent to afford 1-bromo-4-(1-diazo-2,2,2-trifluoroethyl)benzene (3r) as a orange oil (146 mg, $0.551 \mathrm{mmol}, 22 \%$ ). The compound was kept as a $0.6 \mathrm{M}$ solution in DCM at $-18{ }^{\circ} \mathrm{C} .{ }^{1} \mathrm{H}$ NMR $\left(400 \mathrm{MHz}, \mathrm{CDCl}_{3}\right) \delta 7.56-7.48(\mathrm{~m}, 2 \mathrm{H}, \mathrm{ArH}), 7.01-6.91(\mathrm{~m}, 2 \mathrm{H}, \mathrm{ArH}) ;{ }^{13} \mathrm{C} \mathrm{NMR}\left(101 \mathrm{MHz}, \mathrm{CDCl}_{3}\right) \delta$ 
132.8, 125.7 (q, $J=270.3 \mathrm{~Hz}), 124.0,123.0,119.7 ;{ }^{19} \mathrm{~F} \mathrm{NMR}\left(376 \mathrm{MHz}, \mathrm{CDCl}_{3}\right) \delta$-57.5. One carbon was not resolved at $101 \mathrm{MHz}$. The values of the NMR spectra are in accordance with reported literature data. ${ }^{18}$ 


\section{Preparation of EBX reagents}

1,1,1,3,3,3-Hexafluoro-2-(2-iodophenyl)propan-2-ol (11)<smiles>OC(c1ccccc1)(c1ccccc1)C(F)(F)F</smiles>

52

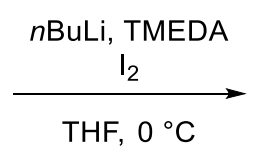

$\mathrm{THF}, 0^{\circ} \mathrm{C}$<smiles>OC(c1ccccc1F)(c1ccccc1I)C(F)(F)F</smiles>

11

Following a reported procedure, ${ }^{19}$ TMEDA ( $1.27 \mathrm{~mL}, 8.40 \mathrm{mmol}, 0.20$ equiv.) was added to a solution of $n$-BuLi ( $37.0 \mathrm{~mL}, 92.0 \mathrm{mmol}, 2.20$ equiv., $2.5 \mathrm{M}$ in hexanes). After $15 \mathrm{~min}$, the cloudy solution was cooled to $0^{\circ} \mathrm{C}$ and 1,1,1,3,3,3-hexafluoro-2-phenylpropan-2-ol (52) (7.07 mL, $42.0 \mathrm{mmol}, 1.00$ equiv.) in THF $(6.0 \mathrm{~mL})$ was added dropwise. The reaction was stirred $30 \mathrm{~min}$ at $0{ }^{\circ} \mathrm{C}$ and then $18 \mathrm{~h}$ at room temperature. Then, THF $(30.0 \mathrm{~mL})$ was added, followed by the portionwise addition of $\mathrm{I}_{2}(11.3 \mathrm{~g}, 44.5$ mmol, 1.05 equiv.) at $0{ }^{\circ} \mathrm{C}$ and the mixture was stirred at $0{ }^{\circ} \mathrm{C}$ for $30 \mathrm{~min}$ and $4 \mathrm{~h}$ at room temperature. The reaction was quenched with saturated aqueous $\mathrm{NH}_{4} \mathrm{Cl}(50 \mathrm{~mL})$ and extracted with diethyl ether (3 $x 30 \mathrm{~mL}$ ). The combined organic phases were washed with water, brine, dried over $\mathrm{MgSO}_{4}$, filtered and the solvent was removed under reduced pressure. The crude product was purified by column chromatography using EtOAc/pentane $3: 97$ as eluent to afford 1,1,1,3,3,3-hexafluoro-2-(2iodophenyl)propan-2-ol (11) as a colorless oil (13.9 g, $37.5 \mathrm{mmol}, 89 \%) .{ }^{1} \mathrm{H}$ NMR $\left(400 \mathrm{MHz}, \mathrm{CDCl}_{3}\right) \delta$ $8.13(\mathrm{dd}, J=7.9,1.4 \mathrm{~Hz}, 1 \mathrm{H}, \operatorname{ArH}), 7.63(\mathrm{~d}, J=8.2 \mathrm{~Hz}, 1 \mathrm{H}, \operatorname{ArH}), 7.43(\mathrm{dt}, J=8.4,1.4 \mathrm{~Hz}, 1 \mathrm{H}, \operatorname{ArH}), 7.11$ $(\mathrm{dt}, J=8.0,1.5 \mathrm{~Hz}, 1 \mathrm{H}, \mathrm{ArH}), 4.23(\mathrm{~s}, 1 \mathrm{H}, \mathrm{OH}) ;{ }^{13} \mathrm{C} \mathrm{NMR}\left(101 \mathrm{MHz}, \mathrm{CDCl}_{3}\right) \delta 144.7,131.4,130.0,129.7$, 128.0, 122.6 (q, $J=291.4 \mathrm{~Hz}), 90.6,78.9(\mathrm{q}, J=32.1 \mathrm{~Hz}) ;{ }^{19} \mathrm{~F} \mathrm{NMR}\left(376 \mathrm{MHz}, \mathrm{CDCl}_{3}\right) \delta$-73.4. The values of the NMR spectra are in accordance with reported literature data. ${ }^{19}$

\section{3,3-Bis(trifluoromethyl)-1 $\lambda^{3}$-benzo[d][1,2]iodaoxol-1(3H)-yl acetate (53)}<smiles>OC(c1ccccc1F)(c1ccccc1I)C(F)(F)F</smiles>

11

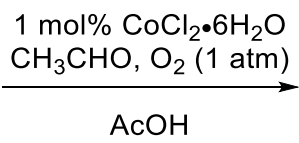<smiles>CC(=O)OI1OC(c2ccccc2)(C(F)(F)F)c2ccccc21</smiles>

53

Following a slightly modified procedure,$^{20}$ a $500 \mathrm{~mL}$ flsak was charged with glacial acetic acid (188 mL), 1,1,1,3,3,3-hexafluoro-2-(2-iodophenyl)propan-2-ol (11) (13.9 g, $37.5 \mathrm{mmol}, 1.00$ equiv.) and cobalt(II) chloride hexahydrate $\left(89.0 \mathrm{~g}, 0.375 \mathrm{mmol}, 0.01\right.$ equiv.). The reaction vessel was purged with $\mathrm{O}_{2}$ for 5 min before acetaldehyde ( $21.4 \mathrm{~mL}, 379 \mathrm{mmol}, 10.0$ equiv.) was added in one portion. The reaction mixture was stirred under $1 \mathrm{~atm}$ of $\mathrm{O}_{2}$, delivered by inflated balloon, at room temperature for $12 \mathrm{~h}$. Acetaldehyde ( $21.4 \mathrm{~mL}, 379 \mathrm{mmol}, 10.00$ equiv.) was added and the reaction continue for $6 \mathrm{~h}$. The solvent was removed under reduced pressure and the residue was dissolved in DCM. The organic layer was washed with distilled water $(50 \mathrm{~mL})$ and extracted with DCM $(3 \times 50 \mathrm{~mL})$. The organic layer was dried over $\mathrm{MgSO}_{4}$, filtered and the solvent was removed under reduced pressure. The obtained residue was triturated in pentane for $0.5 \mathrm{~h}$, filtered and washed with pentane (operation repeated 2 times) to afford 3,3-bis(trifluoromethyl)-1 $\lambda^{3}$-benzo[d] [1,2]iodaoxol-1(3H)-yl acetate (53) as a white solid (9.91 g, $23.2 \mathrm{mmol}, 62 \%) .{ }^{1} \mathrm{H}$ NMR $\left(400 \mathrm{MHz}, \mathrm{DMSO}-d_{6}\right) \delta 7.93$ (ddd, $J=8.4,7.1,1.6 \mathrm{~Hz}, 1 \mathrm{H}, \mathrm{ArH}$ ), $7.85-7.69$ $(\mathrm{m}, 3 \mathrm{H}, \mathrm{ArH}), 2.19\left(\mathrm{~s}, 3 \mathrm{H},(\mathrm{O}) \mathrm{CCH}_{3}\right) ;{ }^{13} \mathrm{C}$ NMR $\left(101 \mathrm{MHz}, \mathrm{DMSO}-d_{6}\right) \delta 174.4,134.2,131.4,131.0,130.8$, $129.5-129.0(\mathrm{~m}), 123.1(\mathrm{q}, J=289.5 \mathrm{~Hz}), 116.1,84.5-83.7(\mathrm{~m}), 20.0 ;{ }^{19} \mathrm{~F}$ NMR $\left(376 \mathrm{MHz}, \mathrm{DMSO}-d_{6}\right) \delta$ -75.1. The values of the NMR spectra are in accordance with reported literature data. ${ }^{21}$

\footnotetext{
19 J. Cvengroš, D. Stolz and A. Togni, Synthesis 2009, 2818.

${ }^{20}$ A. Maity, S.-M. Hyun and D. C. Powers, Nat. Chem. 2018, 10, 200.

${ }^{21}$ P. Eisenberger, S. Gischig and A. Togni, Chem. Eur J. 2006, 12, 2579.
} 
Tert-butyldiphenyl((6-(trimethylsilyl)hex-5-yn-1-yl)oxy)silane (56g)

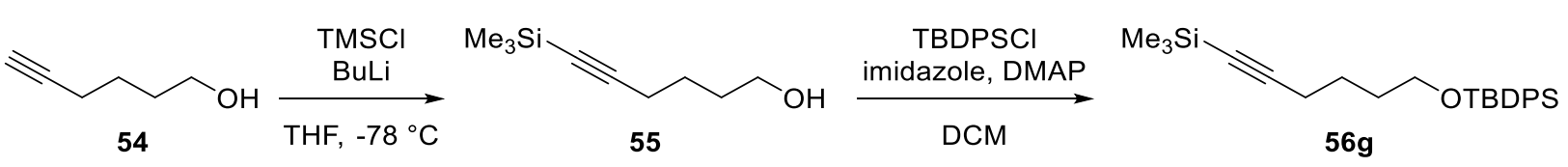

Following a reported procedure, ${ }^{22}$ butyllithium (17.6 mL, $44.0 \mathrm{mmol}, 2.20$ equiv., $2.5 \mathrm{M}$ in hexanes) was added dropwise to a stirring solution of 5-hexyn-1-ol (54) $(2.20 \mathrm{~mL}, 20.0 \mathrm{mmol}, 1.00$ equiv. $)$ in THF $(40.0 \mathrm{~mL})$ at $-78^{\circ} \mathrm{C}$. Stirring was continued for $1 \mathrm{~h}$, then chlorotrimethylsilane $(5.58 \mathrm{~mL}, 44.0 \mathrm{mmol}$, 2.20 equiv.) was added at $-78^{\circ} \mathrm{C}$. After $1 \mathrm{~h}$, the reaction mixture was warmed to $0{ }^{\circ} \mathrm{C}$. Aqueous $1 \mathrm{M} \mathrm{HCl}$ $(30 \mathrm{~mL})$ was added dropwise and stirring was continued for $30 \mathrm{~min}$ at room temperature. The reaction mixture was extracted with diethyl ether $(2 \times 10 \mathrm{~mL})$. The combined organic layers were washed with water $(30 \mathrm{~mL})$ and brine $(10 \mathrm{~mL})$, dried over anhydrous $\mathrm{MgSO}_{4}$, filtered and the solvent was removed under reduced pressure. The crude oil was purified by column chromatography using $\mathrm{Et}_{2} \mathrm{O} /$ pentane 20:80 as eluent to afford 6-(trimethylsilyl)hex-5-yn-1-ol (55) as a colorless oil (2.12 g, $14.2 \mathrm{mmol}, 71 \%)$. ${ }^{1} \mathrm{H} \mathrm{NMR}\left(400 \mathrm{MHz}, \mathrm{CDCl}_{3}\right) \delta 3.73-3.62\left(\mathrm{~m}, 2 \mathrm{H}, \mathrm{CH}_{2} \mathrm{OH}\right), 2.27\left(\mathrm{t}, \mathrm{J}=6.8 \mathrm{~Hz}, 2 \mathrm{H}, \mathrm{C} \equiv \mathrm{CCH}_{2}\right), 1.80-1.52(\mathrm{~m}$, $4 \mathrm{H}, \mathrm{CH}_{2} \mathrm{CH}_{2}$ ), 1.13 (br s, $\left.1 \mathrm{H}, \mathrm{OH}\right), 0.14$ (s, 9H, TMS); $\left.{ }^{13} \mathrm{C} \mathrm{NMR} \mathrm{(101} \mathrm{MHz,} \mathrm{CDCl}_{3}\right) \delta$ 107.3, 84.9, 62.6, 32.0, $25.0,19.8,0.3$. The values of the NMR spectra are in accordance with reported literature data. ${ }^{22}$

Following a reported procedure, ${ }^{23}$ under an atmosphere of nitrogen, 6-(trimethylsilyl)hex-5-yn-1-ol (55) $(511 \mathrm{mg}, 3.00 \mathrm{mmol}, 1.00$ equiv.) was dissolved in DCM $(10.00 \mathrm{~mL})$. The alcohol was then treated, in succession, with imidazole (306 mg, $4.50 \mathrm{mmol}, 1.50$ equiv.), DMAP (110 mg, $0.900 \mathrm{mmol}, 0.3$ equiv.), and tert-butylchlorodiphenylsilane ( $1.17 \mathrm{~mL}, 4.50 \mathrm{mmol}, 1.50$ equiv.). The reaction was stirred at room temperature. After 1 hour, the reaction was diluted with $30 \mathrm{~mL}$ of water then extracted with $\mathrm{DCM}(2 \times 30 \mathrm{~mL})$. The combined organic extracts were dried over anhydrous $\mathrm{MgSO}_{4}$, filtered, and the solvent was removed under reduced pressure. The crude oil was purified by column chromatography using EtOAc/pentane 2:98 as eluent to afford tert-butyldiphenyl((6-(trimethylsilyl)hex-5-yn-1yl)oxy)silane (56g) as a colorless oil $(1.21 \mathrm{~g}, 2.95 \mathrm{mmol}, 98 \%) .{ }^{1} \mathrm{H} \mathrm{NMR}\left(400 \mathrm{MHz}, \mathrm{CDCl}_{3}\right) \delta 7.70-7.62$ $(\mathrm{m}, 4 \mathrm{H}, \mathrm{ArH}), 7.45-7.34(\mathrm{~m}, 6 \mathrm{H}, \mathrm{ArH}), 3.67\left(\mathrm{t}, J=5.9 \mathrm{~Hz}, 2 \mathrm{H}, \mathrm{CH}_{2} \mathrm{O}\right), 2.23\left(\mathrm{t}, J=6.6 \mathrm{~Hz}, 2 \mathrm{H}, \mathrm{C} \equiv \mathrm{CCH}_{2}\right)$, 1.72 - $1.56\left(\mathrm{~m}, 4 \mathrm{H}, \mathrm{CH}_{2} \mathrm{CH}_{2}\right), 1.05$ (s, 9H, tBu), 0.14 (s, 9H, TMS); $\left.{ }^{13} \mathrm{C} \mathrm{NMR} \mathrm{(101} \mathrm{MHz,} \mathrm{CDCl}_{3}\right) \delta 135.7$, $134.2,129.7,127.8,107.6,84.6,63.6,31.8,27.0,25.3,19.8,19.4,0.3$. The values of the NMR spectra are in accordance with reported literature data. ${ }^{23}$

\section{2-(6-(Trimethylsilyl)hex-5-yn-1-yl)isoindoline-1,3-dione (56k)}<smiles>O=C1NC(=O)c2ccccc21</smiles>

$57 \mathrm{O}$

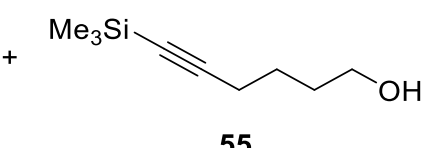

55
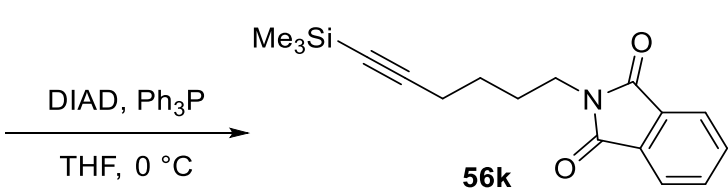

To a stirring solution of 6-(trimethylsilyl)hex-5-yn-1-ol (55) $(852 \mathrm{mg}, 5.00 \mathrm{mmol}, 1.00$ equiv.) in THF $(16.7 \mathrm{~mL})$ was added triphenylphosphine $(1.44 \mathrm{~g}, 5.50 \mathrm{mmol}, 1.10$ equiv.) and DIAD (1.15 mL, 5.50 mmol, 1.10 equiv.) at $0{ }^{\circ} \mathrm{C}$. The reaction mixture was stirred at this temperature for $15 \mathrm{~min}$ and then, phthalimide (57) (750 mg, $5.10 \mathrm{mmol}, 1.02$ equiv.) was added. The reaction was continued at room temperature for $5 \mathrm{~h}$, then cold water $(20 \mathrm{~mL})$ was added and the product was extracted with EtOAc ( 3 $x 40 \mathrm{~mL}$ ). The combined organic layers were washed with brine $(50 \mathrm{~mL})$, dried over anhydrous $\mathrm{MgSO}_{4}$ and the solvent was removed under reduced pressure. The crude product was purified by column chromatography using $\mathrm{Et}_{2} \mathrm{O} /$ pentane $10: 90$ as eluent to furnish 2-(6-(trimethylsilyl)hex-5-yn-1yl)isoindoline-1,3-dione (56k) as a white solid (1.37 g, $4.58 \mathrm{mmol}, 92 \%)$. M.p. $68-70{ }^{\circ} \mathrm{C} ; \mathrm{R}_{\mathrm{f}}=0.26$

\footnotetext{
${ }^{22}$ M. Gersch, F. Gut, V. S. Korotkov, J. Lehmann, T. Böttcher, M. Rusch, C. Hedberg, H. Waldmann, G. Klebe and S. A. Sieber, Angew. Chem. Int. Ed. 2013, 52, 3009.

${ }^{23}$ E. C. McLaughlin and M. P. Doyle, J. Org. Chem. 2008, 73, 4317.
} 
(Et ${ }_{2} \mathrm{O} /$ pentane 10:90); ${ }^{1} \mathrm{H}$ NMR $\left(400 \mathrm{MHz}, \mathrm{CDCl}_{3}\right) \delta 7.83$ (dd, $\left.J=5.4,3.1 \mathrm{~Hz}, 2 \mathrm{H}, \operatorname{ArH}\right), 7.70$ (dd, $J=5.4$, $3.1 \mathrm{~Hz}, 2 \mathrm{H}, \mathrm{ArH}$ ), $3.70\left(\mathrm{t}, \mathrm{J}=7.0 \mathrm{~Hz}, 2 \mathrm{H}, \mathrm{CH}_{2} \mathrm{NPhth}\right), 2.27\left(\mathrm{t}, \mathrm{J}=7.1 \mathrm{~Hz}, 2 \mathrm{H}, \mathrm{C} \equiv \mathrm{CCH}_{2}\right), 1.85-1.74(\mathrm{~m}, 2 \mathrm{H}$, $\left.\mathrm{CH}_{2}\right), 1.61$ - $1.49\left(\mathrm{~m}, 2 \mathrm{H}, \mathrm{CH}_{2}\right), 0.12$ (s, 9H, TMS); ${ }^{13} \mathrm{C} \mathrm{NMR}\left(101 \mathrm{MHz}, \mathrm{CDCl}_{3}\right) \delta$ 168.5, 134.0, 132.3, 123.3, 106.7, 85.1, 37.6, 27.8, 25.9, 19.6, 0.3; IR ( $\left.v_{\max }, \mathrm{cm}^{-1}\right) 2955(\mathrm{w}), 2931$ (w), $2170(\mathrm{w}), 1770(\mathrm{w}), 1705(\mathrm{~m})$, $1440(\mathrm{w}), 1390(\mathrm{~s}), 1352(\mathrm{~m}), 1324(\mathrm{~m}), 1247(\mathrm{~m}), 1035(\mathrm{~m}), 904(\mathrm{~m}), 841(\mathrm{~s}), 761(\mathrm{~s}), 718(\mathrm{~s}), 638(\mathrm{~m})$; HRMS (ESI/QTOF) m/z: [M+H] ${ }^{+}$Calcd. for $\mathrm{C}_{17} \mathrm{H}_{22} \mathrm{NO}_{2} \mathrm{Si}^{+} 300.1414$; Found 300.1413.

General procedure C: Synthesis of EBX reagents:

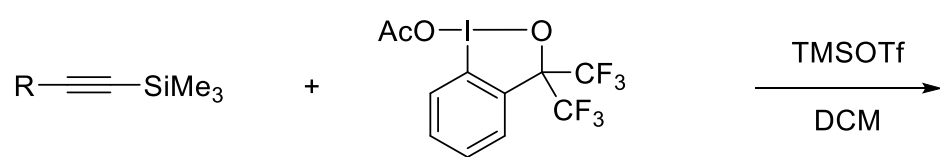

$56 a-56 k$

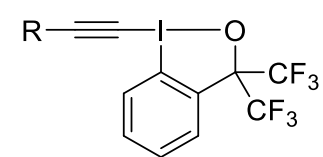

$2 \mathbf{a}-\mathbf{2 k}$

To a solution of 3,3-bis(trifluoromethyl)-1 $\lambda^{3}$-benzo[d][1,2]iodaoxol-1(3H)-yl acetate (53) (1.00 equiv.) in dry DCM $(c=0.2 \mathrm{M})$ was added trimethylsilyl trifluoromethanesulfonate (1.10 equiv.) dropwise at room temperature and the reaction mixture was stirred for $1 \mathrm{~h}$. After this time, the corresponding trimethylethynylsilane (56a-56k) (1.10 equiv.) was added and the mixture was stirred for $6 \mathrm{~h}$ at room temperature. The reaction mixture was then quenched with saturated aqueous $\mathrm{NaHCO}_{3}$ solution and extracted with dichloromethane ( 3 times). The combined organic extracts were dried over $\mathrm{MgSO}_{4}$, filtered and concentrated under reduced pressure. The residue was purified by column chromatography using EtOAc/pentane as eluent to give the corresponding EBX reagent $(\mathbf{2 a}-\mathbf{2 k})$.

((3,3-Bis(trifluoromethyl)-1 $\lambda^{3}$-benzo[d][1,2]iodaoxol-1(3H)-yl)ethynyl)triisopropylsilane (2a)

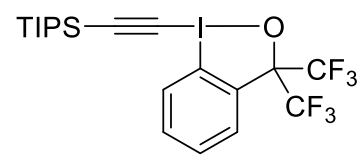

$2 \mathrm{a}$

Following general procedure $C$, starting from triisopropyl((trimethylsilyl)ethynyl)silane (56a) (2.80 g, $11.0 \mathrm{mmol}$ ) and 3,3-bis(trifluoromethyl)-1 $\lambda^{3}$-benzo[d][1,2]iodaoxol-1(3H)-yl acetate (53) $(4.28 \mathrm{~g}, 10.0$ $\mathrm{mmol}), \quad$ afforded (3,3-bis(trifluoromethyl)-1 $\lambda^{3}$-benzo[d][1,2]iodaoxol-1(3H)yl)ethynyl)triisopropylsilane (2a) as a white solid (5.33 g, $9.68 \mathrm{mmol}, 97 \%) .{ }^{1} \mathrm{H} \mathrm{NMR}\left(400 \mathrm{MHz}, \mathrm{CDCl}_{3}\right)$ $\delta 8.36(\mathrm{dd}, J=7.9,1.5 \mathrm{~Hz}, 1 \mathrm{H}, \mathrm{ArH}), 7.88-7.81(\mathrm{~m}, 1 \mathrm{H}, \mathrm{ArH}), 7.74-7.62(\mathrm{~m}, 2 \mathrm{H}, \mathrm{ArH}), 1.23-1.07(\mathrm{~m}$, $21 \mathrm{H}, \mathrm{TIPS}) ;{ }^{13} \mathrm{C}$ NMR $\left(101 \mathrm{MHz}, \mathrm{CDCl}_{3}\right) \delta 132.9,131.3,130.1,130.2-130.0(\mathrm{~m}), 128.3,123.7$ (q, $\mathrm{J}=$ $290.4 \mathrm{~Hz}), 112.3,111.0,81.6(\mathrm{p}, J=29.5 \mathrm{~Hz}), 69.9,18.7,11.4 ;{ }^{19} \mathrm{~F} \mathrm{NMR}\left(376 \mathrm{MHz}, \mathrm{CDCl}_{3}\right) \delta$-76.2. The values of the NMR spectra are in accordance with reported literature data. ${ }^{24}$

\section{1-(5-Chloropent-1-yn-1-yl)-3,3-bis(trifluoromethyl)-1,3-dihydro-1 $\lambda^{3}$-benzo[d][1,2]iodaoxole (2b)}

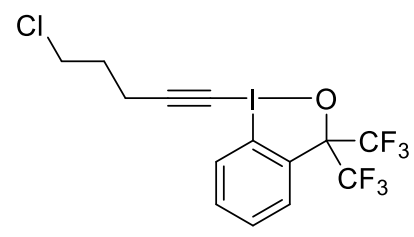

$2 b$

Following general procedure $C$, starting from (5-chloropent-1-yn-1-yl)trimethylsilane (56b) $(197 \mu \mathrm{L}$, $1.10 \mathrm{mmol}$ ) and 3,3-bis(trifluoromethyl)-1 $\lambda^{3}$-benzo[d][1,2]iodaoxol-1(3H)-yl acetate (53) (428 mg, 1.00 $\mathrm{mmol})$ afforded 1-(5-chloropent-1-yn-1-yl)-3,3-bis(trifluoromethyl)-1,3-dihydro-1 $\lambda^{3}$ -

${ }^{24}$ Y. Li, J. P. Brand and J. Waser, Angew. Chem. Int. Ed. 2013, 52, 6743. 
benzo[d][1,2]iodaoxole (2b) as a white solid (273 mg, $0.580 \mathrm{mmol}, 58 \%)$. M.p. $113-115{ }^{\circ} \mathrm{C} ; \mathrm{R}_{\mathrm{f}}=0.47$ (EtOAc/pentane 15:85); ${ }^{1} \mathrm{H}$ NMR (400 MHz, $\left.\mathrm{CDCl}_{3}\right) \delta 8.25-8.17(\mathrm{~m}, 1 \mathrm{H}, \mathrm{ArH}), 7.87-7.78(\mathrm{~m}, 1 \mathrm{H}, \mathrm{ArH})$, $7.73-7.64(\mathrm{~m}, 2 \mathrm{H}, \mathrm{ArH}), 3.70\left(\mathrm{t}, J=6.1 \mathrm{~Hz}, 2 \mathrm{H}, \mathrm{CH}_{2} \mathrm{Cl}\right), 2.74\left(\mathrm{t}, J=6.9 \mathrm{~Hz}, 2 \mathrm{H}, \mathrm{CH}_{2} \mathrm{C} \equiv \mathrm{C}\right), 2.07(\mathrm{p}, J=6.6$ $\left.\mathrm{Hz}, 2 \mathrm{H}, \mathrm{CH}_{2} \mathrm{CH}_{2} \mathrm{Cl}\right) ;{ }^{13} \mathrm{C} \mathrm{NMR}\left(101 \mathrm{MHz}, \mathrm{CDCl}_{3}\right) \delta$ 133.0, 131.3, 130.1, 130.1-129.9 (m), 128.3, 123.7 (q, $J=290.5 \mathrm{~Hz}), 111.0,105.5,81.7(\mathrm{p}, J=29.6 \mathrm{~Hz}), 45.2,43.5,31.0,17.8 ;{ }^{19} \mathrm{~F} \mathrm{NMR}\left(376 \mathrm{MHz}, \mathrm{CDCl}_{3}\right) \delta-$ 76.2; IR ( $\left.v_{\max }, \mathrm{cm}^{-1}\right) 2158(\mathrm{w}), 1441(\mathrm{w}), 1427(\mathrm{w}), 1263(\mathrm{~s}), 1178(\mathrm{~s}), 1145$ (s), $966(\mathrm{~s}), 946$ (s), $768(\mathrm{~s})$, 753 (s), 729 (s), 660 (m); HRMS (ESI/QTOF) m/z: [M+H] ${ }^{+}$Calcd. for $\mathrm{C}_{14} \mathrm{H}_{11} \mathrm{ClF}_{6} \mathrm{IO} \mathrm{O}^{+}$470.9442; Found 470.9446 .

1-(Hex-1-yn-1-yl)-3,3-bis(trifluoromethyl)-1,3-dihydro-1 $\lambda^{3}$-benzo[d][1,2]iodaoxole (2c)

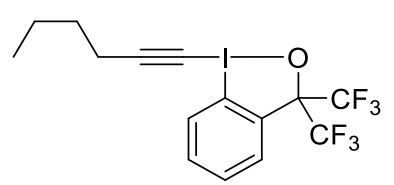

2c

Following general procedure $C$, starting from hex-1-yn-1-yltrimethylsilane (56c) $(222 \mu \mathrm{L}, 1.10 \mathrm{mmol})$ and 3,3-bis(trifluoromethyl)- $1 \lambda^{3}$-benzo[d][1,2]iodaoxol-1(3H)-yl acetate (53) (428 mg, $1.00 \mathrm{mmol}$ ), afforded 1-(hex-1-yn-1-yl)-3,3-bis(trifluoromethyl)-1,3-dihydro-1 $\lambda^{3}$-benzo[d][1,2]iodaoxole (2c) as a white solid (285 mg, $0.630 \mathrm{mmol}, 63 \%) .{ }^{1} \mathrm{H}$ NMR (400 MHz, CDCl 3$) \delta 8.26-8.18(\mathrm{~m}, 1 \mathrm{H}, \mathrm{ArH}), 7.86-$ $7.79(\mathrm{~m}, 1 \mathrm{H}, \mathrm{ArH}), 7.73-7.64(\mathrm{~m}, 2 \mathrm{H}, \mathrm{ArH}), 2.53\left(\mathrm{t}, J=7.0 \mathrm{~Hz}, 2 \mathrm{H}, \mathrm{CH}_{2} \mathrm{C} \equiv \mathrm{C}\right), 1.67-1.56(\mathrm{~m}, 2 \mathrm{H}$, $\left.\mathrm{CH}_{2} \mathrm{CH}_{2} \mathrm{C} \equiv \mathrm{C}\right), 1.53-1.42\left(\mathrm{~m}, 2 \mathrm{H}, \mathrm{CH}_{2} \mathrm{CH}_{3}\right), 0.96\left(\mathrm{t}, J=7.3 \mathrm{~Hz}, 3 \mathrm{H}, \mathrm{CH}_{2} \mathrm{CH}_{3}\right) ;{ }^{13} \mathrm{C} \mathrm{NMR}\left(101 \mathrm{MHz}, \mathrm{CDCl}_{3}\right) \delta$ $132.9,131.2,130.2,130.0-129.8(\mathrm{~m}), 128.3,123.8(\mathrm{q}, J=290.7 \mathrm{~Hz}), 111.1,108.1,81.7(\mathrm{p}, J=29.4 \mathrm{~Hz})$, $43.5,30.6,22.2,20.2,13.7 ;{ }^{19} \mathrm{~F} \mathrm{NMR}\left(376 \mathrm{MHz}, \mathrm{CDCl}_{3}\right) \delta$-76.2. The values of the NMR spectra are in accordance with reported literature data. ${ }^{25}$

1-(Cyclopropylethynyl)-3,3-bis(trifluoromethyl)-1,3-dihydro-1 $\lambda^{3}$-benzo[d][1,2]iodaoxole (2d)

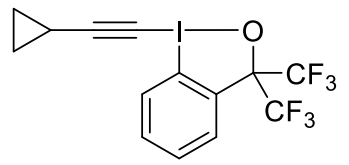

2d

Following general procedure $\mathrm{C}$, starting from (cyclopropylethynyl)trimethylsilane (56d) $(995 \mu \mathrm{L}, 5.50$ $\mathrm{mmol}$ ) and 3,3-bis(trifluoromethyl)-1 $\lambda^{3}$-benzo[d][1,2]iodaoxol-1(3H)-yl acetate (53) (2.14 g, 5.00 $\mathrm{mmol}$ ), afforded 1-(cyclopropylethynyl)-3,3-bis(trifluoromethyl)-1,3-dihydro-1 $\lambda^{3}$ benzo[d][1,2]iodaoxole (2d) as an off-white solid $\left.(873 \mathrm{mg}, 2.01 \mathrm{mmol}, 40 \%) .{ }^{1} \mathrm{H} \mathrm{NMR} \mathrm{(400} \mathrm{MHz}, \mathrm{CDCl}_{3}\right)$ $\delta 8.32-8.14(\mathrm{~m}, 1 \mathrm{H}, \mathrm{ArH}), 7.88-7.74(\mathrm{~m}, 1 \mathrm{H}, \mathrm{ArH}), 7.74-7.59(\mathrm{~m}, 2 \mathrm{H}, \operatorname{ArH}), 1.54(\mathrm{tt}, \mathrm{J}=8.2,5.0 \mathrm{~Hz}$, $1 \mathrm{H}, \mathrm{CHC} \equiv \mathrm{C}), 1.00-0.91$ (m, 2H, CH -cyclopropyl), $0.91-0.85$ (m, 2H, CH -cyclopropyl); ${ }^{13} \mathrm{C}$ NMR (101 $\left.\mathrm{MHz}, \mathrm{CDCl}_{3}\right) \delta 132.9,131.2,130.2,130.0,129.8,128.2,123.8(\mathrm{q}, J=290.8 \mathrm{~Hz}), 81.7(\mathrm{p}, J=29.5 \mathrm{~Hz}$ ), $39.4,9.5,1.0 ;{ }^{19} \mathrm{~F} \mathrm{NMR}\left(376 \mathrm{MHz}, \mathrm{CDCl}_{3}\right) \delta$-76.3. The values of the NMR spectra are in accordance with reported literature data. ${ }^{26}$

\footnotetext{
${ }^{25}$ X. Li, X. Xie, N. Sun and Y. Liu, Angew. Chem. Int. Ed. 2017, 56, 6994.

${ }^{26}$ J. Wu, X. Deng, H. Hirao and N. Yoshikai, J. Am. Chem. Soc. 2016, 138, 9105.
} 


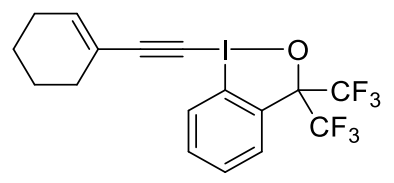

$2 e$

Following general procedure $C$, starting from (cyclohex-1-en-1-ylethynyl)trimethylsilane (56e) $(196 \mathrm{mg}$, $1.10 \mathrm{mmol}$ ) and 3,3-bis(trifluoromethyl)- $1 \lambda^{3}$-benzo[d] [1,2]iodaoxol-1(3H)-yl acetate (53) (428 mg, 1.00 $\mathrm{mmol}$ ), afforded 1-(cyclohex-1-en-1-ylethynyl)-3,3-bis(trifluoromethyl)-1,3-dihydro-1 $\lambda^{3}$ benzo[d] [1,2]iodaoxole (2e) as an off-white solid (213 mg, $0.450 \mathrm{mmol}, 45 \%) .{ }^{1} \mathrm{H} \mathrm{NMR}\left(400 \mathrm{MHz}, \mathrm{CDCl}_{3}\right)$ $\delta 8.29-8.13(\mathrm{~m}, 1 \mathrm{H}, \operatorname{ArH}), 7.91-7.78(\mathrm{~m}, 1 \mathrm{H}, \mathrm{ArH}), 7.73-7.60(\mathrm{~m}, 2 \mathrm{H}, \operatorname{ArH}), 6.36(\mathrm{p}, J=2.2 \mathrm{~Hz}, 1 \mathrm{H}$, $\mathrm{C}=\mathrm{CH}), 2.28-2.13\left(\mathrm{~m}, 4 \mathrm{H}, 2 \times \mathrm{CH}_{2} \mathrm{C}=\mathrm{C}\right), 1.75-1.54\left(\mathrm{~m}, 4 \mathrm{H}, \mathrm{CH}_{2} \mathrm{CH}_{2}\right) ;{ }^{13} \mathrm{C} \mathrm{NMR}\left(101 \mathrm{MHz}, \mathrm{CDCl}_{3}\right) \delta 140.1$, $132.9,129.8,129.6,128.1,127.8,123.4(q, J=290.3 \mathrm{~Hz}), 119.8,111.2,107.7,81.3(p, J=29.6 \mathrm{~Hz}), 50.5$, 28.7, 25.7, 21.9, 20.9; ${ }^{19} \mathrm{~F}$ NMR $\left(376 \mathrm{MHz}, \mathrm{CDCl}_{3}\right) \delta-76.2$. The values of the NMR spectra are in accordance with reported literature data. ${ }^{27}$

\section{1-((2-Bromophenyl)ethynyl)-3,3-bis(trifluoromethyl)-1,3-dihydro-1 $\lambda^{3}$-benzo[d][1,2]iodaoxole (2f)}

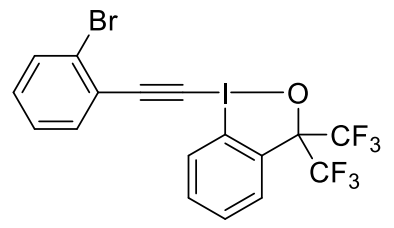

$2 f$

Following general procedure $\mathrm{C}$, starting from ((2-bromophenyl)ethynyl)trimethylsilane (56f) $(234 \mu \mathrm{L}$, $1.10 \mathrm{mmol}$ ) and 3,3-bis(trifluoromethyl)- $1 \lambda^{3}$-benzo[d][1,2]iodaoxol-1(3H)-yl acetate (53) (428 mg, 1.00 $\mathrm{mmol})$ afforded 1-((2-bromophenyl)ethynyl)-3,3-bis(trifluoromethyl)-1,3-dihydro- $1 \lambda^{3}$ benzo[d][1,2]iodaoxole (2f) as a white solid $(535 \mathrm{mg}, 0.970 \mathrm{mmol}, 97 \%) .{ }^{1} \mathrm{H}$ NMR $\left(400 \mathrm{MHz}, \mathrm{CDCl}_{3}\right) \delta$ $8.52-8.43(\mathrm{~m}, 1 \mathrm{H}, \operatorname{ArH}), 7.90-7.81(\mathrm{~m}, 1 \mathrm{H}, \operatorname{ArH}), 7.76-7.68(\mathrm{~m}, 2 \mathrm{H}, \operatorname{Ar} H), 7.66(\mathrm{dd}, J=8.0,1.1 \mathrm{~Hz}$, $1 \mathrm{H}, \operatorname{ArH}), 7.57(\mathrm{dd}, J=7.6,1.8 \mathrm{~Hz}, 1 \mathrm{H}, \mathrm{ArH}), 7.35(\mathrm{td}, J=7.6,1.3 \mathrm{~Hz}, 1 \mathrm{H}, \operatorname{ArH}), 7.32-7.24(\mathrm{~m}, 1 \mathrm{H}, \operatorname{ArH})$; ${ }^{13} \mathrm{C}$ NMR $\left(101 \mathrm{MHz}, \mathrm{CDCl}_{3}\right) \delta 134.5,133.2,132.9,131.4,131.2,130.2-129.9(\mathrm{~m}), 130.0,128.9,127.5$, $126.2,123.9,123.7$ (q, $J=290.6 \mathrm{~Hz}), 111.6,103.0,81.8(\mathrm{p}, J=29.8 \mathrm{~Hz}), 59.6 ;{ }^{19} \mathrm{~F} \mathrm{NMR}\left(376 \mathrm{MHz}, \mathrm{CDCl}_{3}\right)$ $\delta$-76.1. The values of the NMR spectra are in accordance with reported literature data. ${ }^{28}$

((6-(3,3-Bis(trifluoromethyl)-1 $\lambda^{3}$-benzo[d][1,2]iodaoxol-1(3H)-yl)hex-5-yn-1-yl)oxy)(tertbutyl)diphenylsilane $(2 \mathrm{~g})$

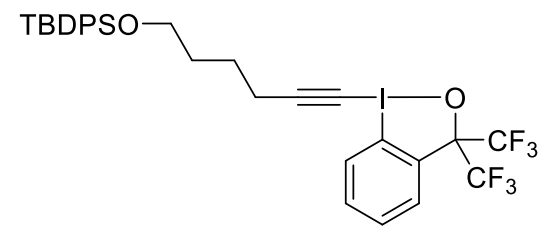

$2 \mathrm{~g}$

Following general procedure $C$, starting from tert-butyldiphenyl((6-(trimethylsilyl)hex-5-yn-1yl)oxy)silane (56g) $(450 \mathrm{mg}, 1.10 \mathrm{mmol})$ and 3,3-bis(trifluoromethyl)-1 $\lambda^{3}$-benzo[d][1,2]iodaoxol-1(3H)yl acetate $(53)(428 \mathrm{mg}, 1.00 \mathrm{mmol})$, afforded ((6-(3,3-bis(trifluoromethyl)-1 $1 \lambda^{3}$-benzo[d][1,2]iodaoxol-

${ }^{27}$ V. V. Zhdankin, C. J. Kuehl, A. P. Krasutsky, J. T. Bolz and A. J. Simonsen, J. Org. Chem. 1996, 61, 6547.

${ }^{28}$ Y. Yang, P. Antoni, M. Zimmer, K. Sekine, F. F. Mulks, L. Hu, L. Zhang, M. Rudolph, F. Rominger and A. S. K. Hashmi, Angew. Chem. Int. Ed. 2019, 58, 5129. 
1(3H)-yl)hex-5-yn-1-yl)oxy)(tert-butyl)diphenylsilane (2g) as a white solid (355 $\mathrm{mg}, 0.500 \mathrm{mmol}, 50 \%$ ). M.p. $110-112{ }^{\circ} \mathrm{C} ; R_{f}=0.40(E t O A c / p e n t a n e ~ 10: 90) ;{ }^{1} \mathrm{H} \mathrm{NMR}\left(400 \mathrm{MHz}, \mathrm{CDCl}_{3}\right) \delta 8.21(\mathrm{dd}, J=8.1,1.3 \mathrm{~Hz}$, $1 \mathrm{H}, \mathrm{ArH}), 7.86-7.78(\mathrm{~m}, 1 \mathrm{H}, \mathrm{ArH}), 7.72-7.58(\mathrm{~m}, 6 \mathrm{H}, \mathrm{ArH}), 7.46-7.32(\mathrm{~m}, 6 \mathrm{H}, \mathrm{ArH}), 3.77-3.66(\mathrm{~m}$, $\left.2 \mathrm{H}, \mathrm{CH} \mathrm{O}_{2} \mathrm{OTBDPS}\right), 2.59-2.49\left(\mathrm{~m}, 2 \mathrm{H}, \mathrm{CH}_{2} \mathrm{C} \equiv \mathrm{C}\right), 1.80-1.65$ (m, 4H, $\left.\mathrm{CH}_{2} \mathrm{CH}_{2} \mathrm{CH}_{2} \mathrm{O}\right), 1.06$ (s, 9H, tBu-Si); ${ }^{13} \mathrm{C}$ NMR $\left(101 \mathrm{MHz}, \mathrm{CDCl}_{3}\right) \delta$ 135.6, 133.8, 132.7, 131.1, 130.0, $129.9-129.7(\mathrm{~m}), 129.7,128.1,127.7$, $123.6(q, J=290.4 \mathrm{~Hz}), 110.9,107.7,81.5(\mathrm{p}, J=29.5 \mathrm{~Hz}), 63.2,43.7,31.6,26.9,25.0,20.2,19.3 ;{ }^{19} \mathrm{~F}$ NMR (376 MHz, CDCl $)_{3}$ ) -76.2; IR $\left(v_{\max }, \mathrm{cm}^{-1}\right) 2934(\mathrm{w}), 2855(\mathrm{w}), 2155(\mathrm{w}), 2071(\mathrm{~m}), 1695(\mathrm{w}), 1427$ $(\mathrm{m}), 1266(\mathrm{~m}), 1257(\mathrm{~m}), 1181(\mathrm{~s}), 1151(\mathrm{~s}), 1107(\mathrm{~m}), 966(\mathrm{~s}), 946(\mathrm{~s}), 762(\mathrm{~s}), 732(\mathrm{~s}), 701$ (s); HRMS (ESI/QTOF) m/z: [M+H] ${ }^{+}$Calcd. for $\mathrm{C}_{31} \mathrm{H}_{32} \mathrm{~F}_{6} \mathrm{IO}_{2} \mathrm{Si}^{+}$705.1115; Found 705.1114.

\section{1-(Phenylethynyl)-3,3-bis(trifluoromethyl)-1,3-dihydro-1 $\lambda^{3}$-benzo[d][1,2]iodaoxole $(2 \mathrm{~h})$}

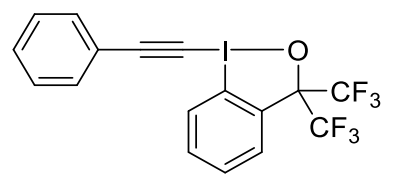

$2 \mathrm{~h}$

Following general procedure $C$, starting from trimethyl(phenylethynyl)silane (56h) (192 mg, 1.10 $\mathrm{mmol}$ ) and 3,3-bis(trifluoromethyl)-1 $\lambda^{3}$-benzo[d][1,2]iodaoxol-1(3H)-yl acetate (53) (428 mg, 1.00 mmol), afforded 1-(phenylethynyl)-3,3-bis(trifluoromethyl)-1,3-dihydro- $1 \lambda^{3}$-benzo[ $\left.d\right][1,2]$ iodaoxole (2h) as a white solid (395 mg, $\left.0.840 \mathrm{mmol}, 84 \%) .{ }^{1} \mathrm{H} \mathrm{NMR} \mathrm{(400} \mathrm{MHz}, \mathrm{CDCl}_{3}\right) \delta 8.34-8.24(\mathrm{~m}, 1 \mathrm{H}, \mathrm{ArH})$, 7.86 (ddt, $J=7.4,3.2,1.4 \mathrm{~Hz}, 1 \mathrm{H}, \mathrm{ArH}), 7.75-7.66(\mathrm{~m}, 2 \mathrm{H}, \mathrm{ArH}), 7.59-7.53(\mathrm{~m}, 2 \mathrm{H}, \mathrm{ArH}), 7.48-7.37$ (m, 3H, ArH); ${ }^{13} \mathrm{C}$ NMR (101 MHz, $\left.\mathrm{CDCl}_{3}\right) \delta 133.1,132.8,131.4,130.3,130.1,130.0,128.8,128.5,123.7$ $(q, J=289.8 \mathrm{~Hz}), 121.4,111.6,105.4,82.5-81.1(\mathrm{~m}), 54.5 ;{ }^{19} \mathrm{~F} \mathrm{NMR}\left(376 \mathrm{MHz}, \mathrm{CDCl}_{3}\right) \delta-76.2$. The values of the NMR spectra are in accordance with reported literature data. ${ }^{29}$

\section{1-(Thiophen-2-ylethynyl)-3,3-bis(trifluoromethyl)-1,3-dihydro-1 $\lambda^{3}$-benzo[d][1,2]iodaoxole (2i)}

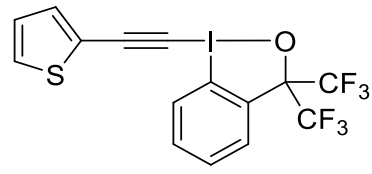

2i

Following general procedure $C$, starting from trimethyl(thiophen-2-ylethynyl)silane (56i) $(182 \mu \mathrm{L}, 1.10$ $\mathrm{mmol}$ ) and 3,3-bis(trifluoromethyl)-1 $\lambda^{3}$-benzo[d][1,2]iodaoxol-1(3H)-yl acetate (53) (428 mg, 1.00 $\mathrm{mmol}$ ), afforded 1-(thiophen-2-ylethynyl)-3,3-bis(trifluoromethyl)-1,3-dihydro- $1 \lambda^{3}$ benzo[d] [1,2]iodaoxole (2i) as an off-white solid (403 mg, $0.850 \mathrm{mmol}, 85 \%) .{ }^{1} \mathrm{H} \mathrm{NMR}\left(400 \mathrm{MHz}, \mathrm{CDCl}_{3}\right)$ $\delta 8.30-8.19(\mathrm{~m}, 1 \mathrm{H}, \mathrm{ArH}), 7.89-7.80(\mathrm{~m}, 1 \mathrm{H}, \mathrm{ArH}), 7.76-7.66(\mathrm{~m}, 2 \mathrm{H}, \operatorname{ArH}), 7.44-7.38(\mathrm{~m}, 2 \mathrm{H}, \operatorname{ArH})$, 7.07 (dd, J = 5.1, 3.7 Hz, $1 \mathrm{H}, \mathrm{ArH}) ;{ }^{13} \mathrm{C} N M R\left(101 \mathrm{MHz}, \mathrm{CDCl}_{3}\right) \delta 135.2,133.2,131.4,130.2,123.0,129.9$, $128.5,127.5,123.7(q, J=291.2 \mathrm{~Hz}), 121.3,111.8,98.4,81.8(\mathrm{p}, J=29.7 \mathrm{~Hz}), 59.7 ;{ }^{19} \mathrm{~F} \mathrm{NMR}(376 \mathrm{MHz}$, $\left.\mathrm{CDCl}_{3}\right) \delta-76.2$. The values of the NMR spectra are in accordance with reported literature data. ${ }^{29}$

\footnotetext{
${ }^{29}$ X. Wu, S. Shirakawa and K. Maruoka, Org. Biomol. Chem. 2014, 12, 5388.
} 


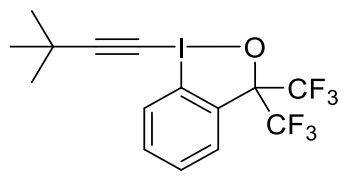

2j

Following general procedure $C$, starting from (3,3-dimethylbut-1-yn-1-yl)trimethylsilane (56j) $(229 \mu \mathrm{L}$, $1.10 \mathrm{mmol}$ ) and 3,3-bis(trifluoromethyl)-1 $\lambda^{3}$-benzo[d] [1,2]iodaoxol-1(3H)-yl acetate (53) $(428 \mathrm{mg}, 1.00$ $\mathrm{mmol}$ ), afforded 1-(3,3-dimethylbut-1-yn-1-yl)-3,3-bis(trifluoromethyl)-1,3-dihydro-1 $\lambda^{3}$ benzo[d][1,2]iodaoxole (2j) as a white solid $(350 \mathrm{mg}, 0.780 \mathrm{mmol}, 78 \%) .{ }^{1} \mathrm{H} \mathrm{NMR}\left(400 \mathrm{MHz}, \mathrm{CDCl}_{3}\right) \delta$ $8.24-8.14(\mathrm{~m}, 1 \mathrm{H}, \mathrm{ArH}), 7.89-7.78(\mathrm{~m}, 1 \mathrm{H}, \mathrm{ArH}), 7.74-7.64(\mathrm{~m}, 2 \mathrm{H}, \mathrm{ArH}), 1.34(\mathrm{~s}, 9 \mathrm{H}, t \mathrm{Bu}) ;{ }^{13} \mathrm{C} \mathrm{NMR}$ $\left(101 \mathrm{MHz}, \mathrm{CDCl}_{3}\right) \delta 132.7,131.1,130.3,130.0,128.0,123.9$ (q, $\left.J=290.3 \mathrm{~Hz}\right), 116.1,111.2,81.9(\mathrm{p}, J=$ $29.6 \mathrm{~Hz}), 42.0,30.8,29.5 ;{ }^{19} \mathrm{~F} \mathrm{NMR}\left(376 \mathrm{MHz}, \mathrm{CDCl}_{3}\right) \delta$-76.2. The values of the NMR spectra are in accordance with reported literature data. ${ }^{27}$

2-(6-(3,3-Bis(trifluoromethyl)-1 $\lambda^{3}$-benzo[d][1,2]iodaoxol-1(3H)-yl)hex-5-yn-1-yl)isoindoline-1,3dione (2k)

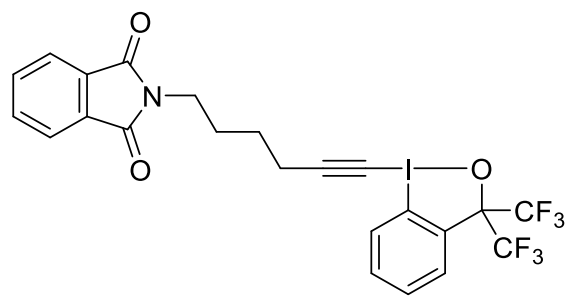

$2 k$

Following general procedure $C$, starting from 2-(6-(trimethylsilyl)hex-5-yn-1-yl)isoindoline-1,3-dione (56k) $(330 \mathrm{mg}, 1.10 \mathrm{mmol})$ and 3,3-bis(trifluoromethyl)-1 $\lambda^{3}$-benzo[d][1,2]iodaoxol-1(3H)-yl acetate (53) $(428 \mathrm{mg}, 1.00 \mathrm{mmol})$, afforded 2-(6-(3,3-bis(trifluoromethyl)-1 $\lambda^{3}$-benzo[d][1,2]iodaoxol-1(3H)yl)hex-5-yn-1-yl)isoindoline-1,3-dione (2k) as a white solid (590 mg, 0.990 mmol, 99\%). M.p. 139-141 ${ }^{\circ} \mathrm{C} ; \mathrm{R}_{\mathrm{f}}=0.13$ (EtOAc/pentane 15:85); ${ }^{1} \mathrm{H}$ NMR $\left(400 \mathrm{MHz}, \mathrm{CDCl}_{3}\right) \delta 8.22-8.15(\mathrm{~m}, 1 \mathrm{H}, \mathrm{ArH}), 7.89-7.78$ $(\mathrm{m}, 3 \mathrm{H}, \mathrm{ArH}), 7.77-7.65(\mathrm{~m}, 4 \mathrm{H}, \mathrm{ArH}), 3.75\left(\mathrm{t}, J=7.0 \mathrm{~Hz}, 2 \mathrm{H}, \mathrm{CH}_{2} \mathrm{NPhth}\right), 2.59\left(\mathrm{t}, J=7.0 \mathrm{~Hz}, 2 \mathrm{H}, \mathrm{CH}_{2} \mathrm{C} \equiv \mathrm{C}\right)$, $1.87\left(\mathrm{tt}, J=7.7,6.4 \mathrm{~Hz}, 2 \mathrm{H}, \mathrm{CH}_{2} \mathrm{CH}_{2} \mathrm{~N}\right), 1.73-1.62\left(\mathrm{~m}, 2 \mathrm{H}, \mathrm{CH}_{2} \mathrm{CH}_{2} \mathrm{C} \equiv \mathrm{C}\right) ;{ }^{13} \mathrm{C} \mathrm{NMR}\left(101 \mathrm{MHz}, \mathrm{CDCl}_{3}\right) \delta$ $168.5,134.2,133.0,132.2,131.2,130.1,130.0-129.8(\mathrm{~m}), 128.4,123.7$ (q, $J=290.4 \mathrm{~Hz}), 123.4,111.0$, 106.9, $81.7(\mathrm{p}, J=29.4 \mathrm{~Hz}), 44.6,37.4,27.8,25.6,20.0 ;{ }^{19} \mathrm{~F} \mathrm{NMR}\left(376 \mathrm{MHz}, \mathrm{CDCl}_{3}\right) \delta-76.2 ; \mathrm{IR}\left(\mathrm{v}_{\max }, \mathrm{cm}^{-}\right.$ 1) $2355(\mathrm{w}), 2164(\mathrm{w}), 2099(\mathrm{w}), 1707(\mathrm{~m}), 1393(\mathrm{~m}), 1264(\mathrm{~m}), 1180(\mathrm{~s}), 1154(\mathrm{~s}), 965(\mathrm{~m}), 947(\mathrm{~s}), 767$ (s), 751 (s), 714 (s) HRMS (ESI/QTOF) m/z: [M+H] $]^{+}$Calcd. for $\mathrm{C}_{23} \mathrm{H}_{17} \mathrm{~F}_{6} \mathrm{INO}_{3}{ }^{+}$596.0152; Found 596.0157. 
7. Spectra of new compounds

${ }^{1} \mathbf{H}-\mathrm{NMR}\left(400 \mathrm{MHz}, \mathrm{CDCl}_{3}\right)$ of compound $\mathbf{5 a}$

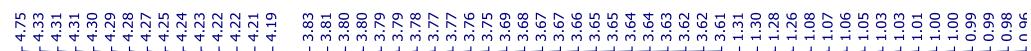
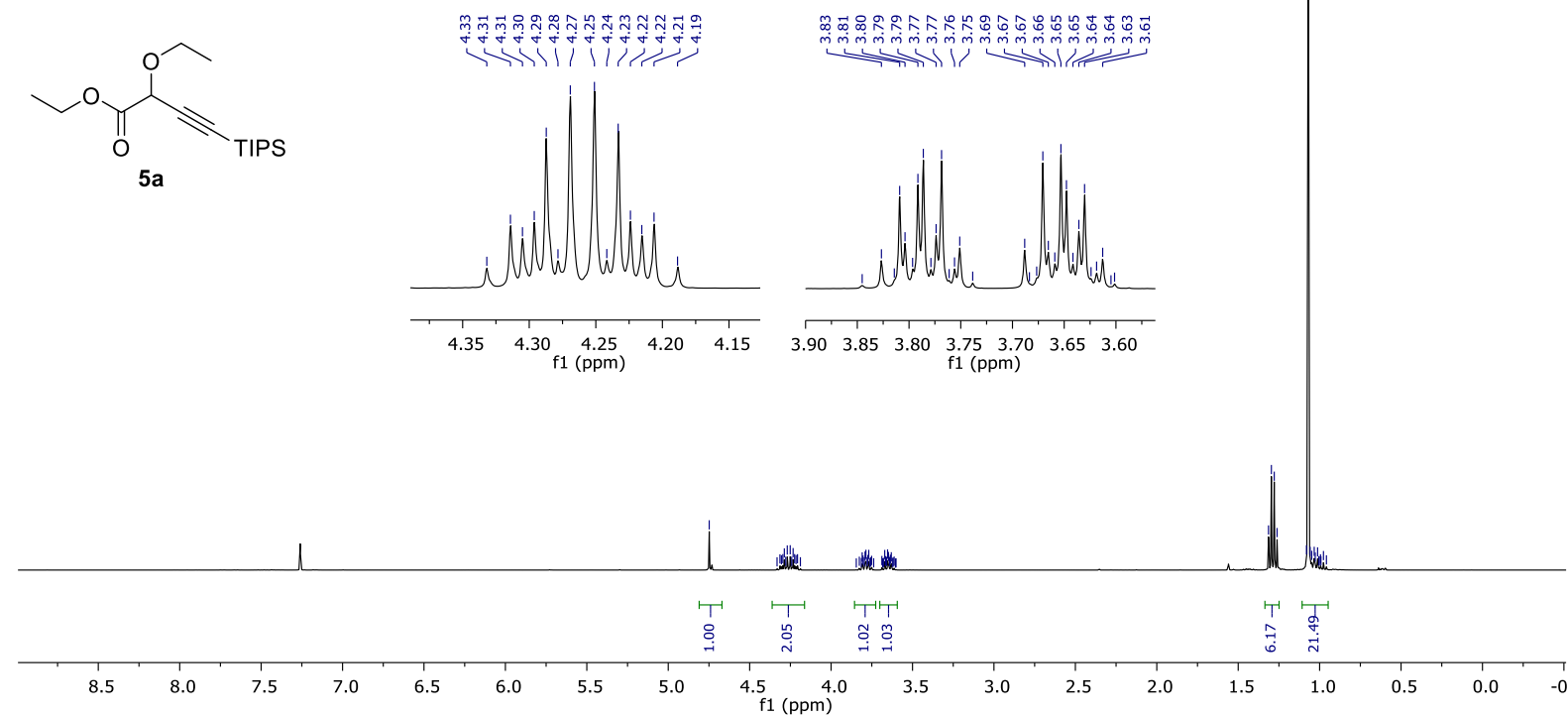

${ }^{13}$ C-NMR (101 MHz, $\mathrm{CDCl}_{3}$ ) of compound 5a 㐫<smiles>CC#CC(OCC)C(=O)OCC</smiles>

$5 a$

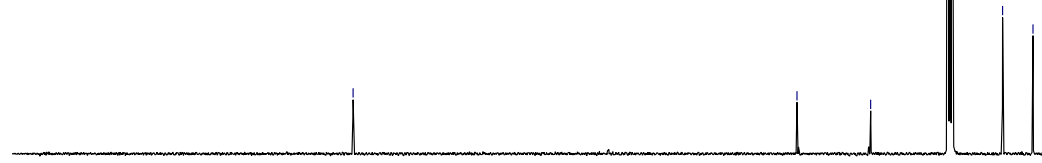


${ }^{1} \mathbf{H}-\mathrm{NMR}\left(400 \mathrm{MHz}, \mathrm{CDCl}_{3}\right)$ of compound $\mathbf{5 b}$

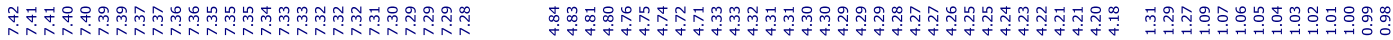
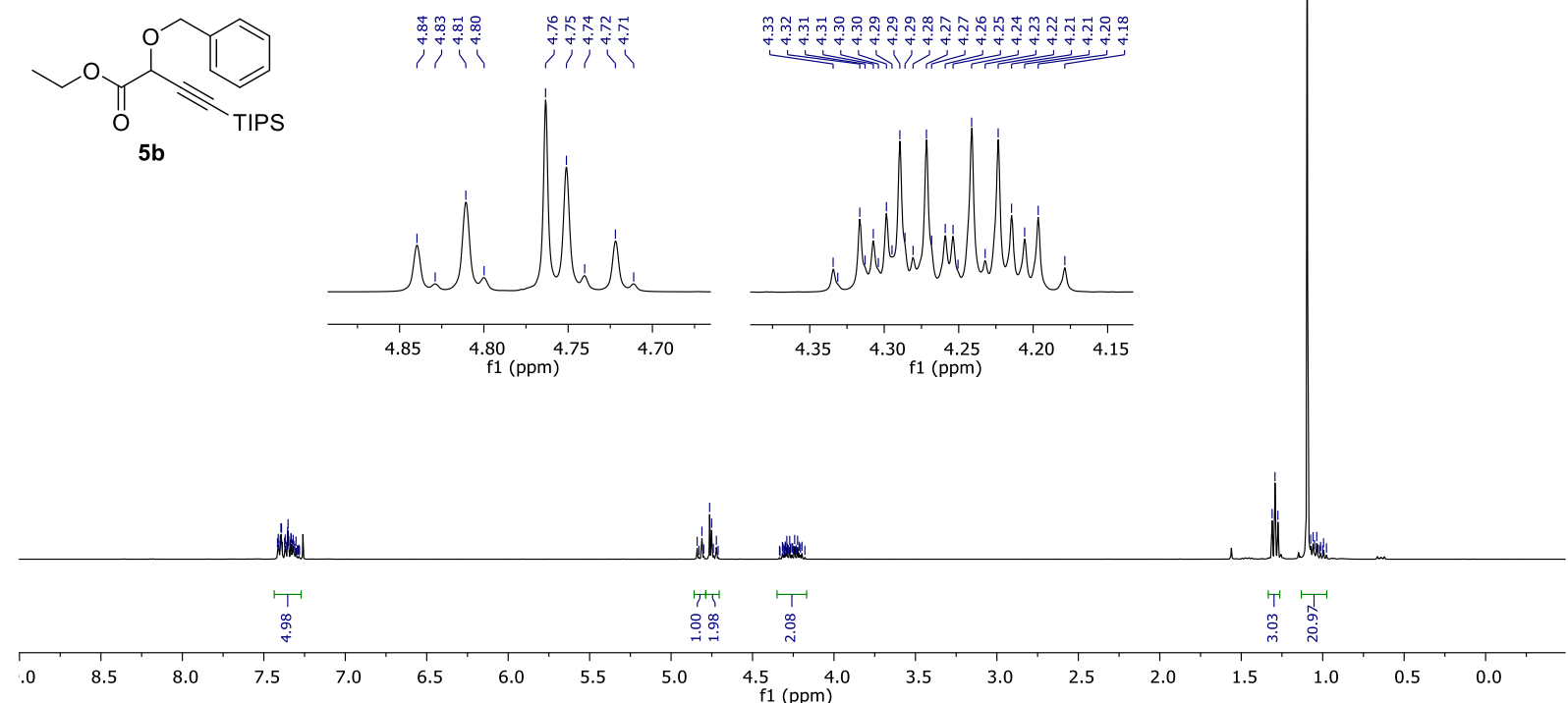

${ }^{13} \mathbf{C}-\mathrm{NMR}\left(101 \mathrm{MHz}, \mathrm{CDCl}_{3}\right)$ of compound $\mathbf{5 b}$

要
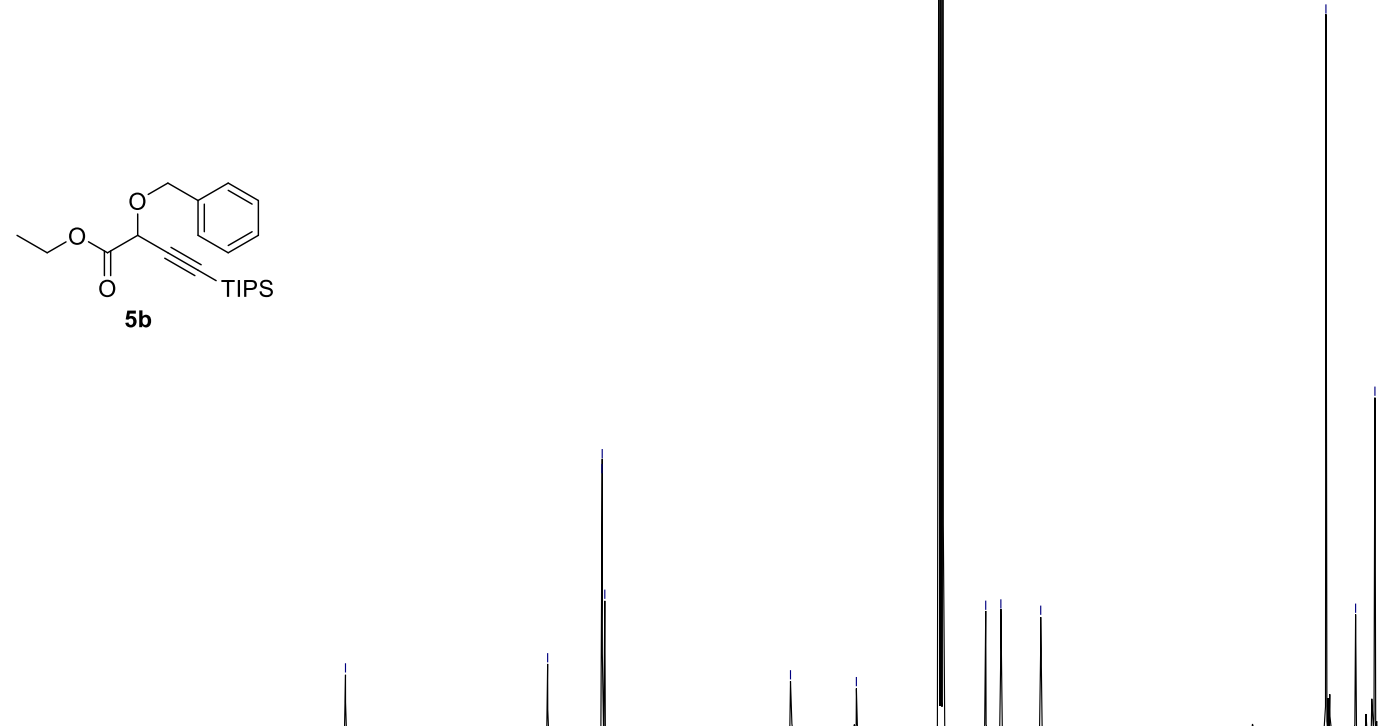

$\begin{array}{llllllllllllllllllllllllll}210 & 200 & 190 & 180 & 170 & 160 & 150 & 140 & 130 & 120 & 110 & \begin{array}{c}100 \\ \mathrm{f} 1(\mathrm{ppm})\end{array} & 90 & 80 & 70 & 60 & 50 & 40 & 30 & 20 & 10 & 0 & -10\end{array}$ 
${ }^{1} \mathrm{H}-\mathrm{NMR}\left(400 \mathrm{MHz}, \mathrm{CDCl}_{3}\right.$ ) of compound $\mathbf{5 c}$

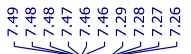

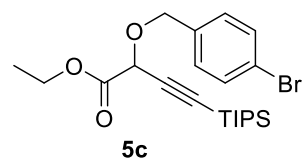

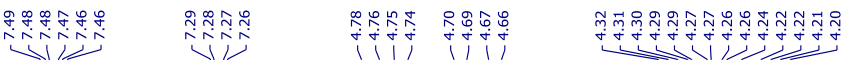
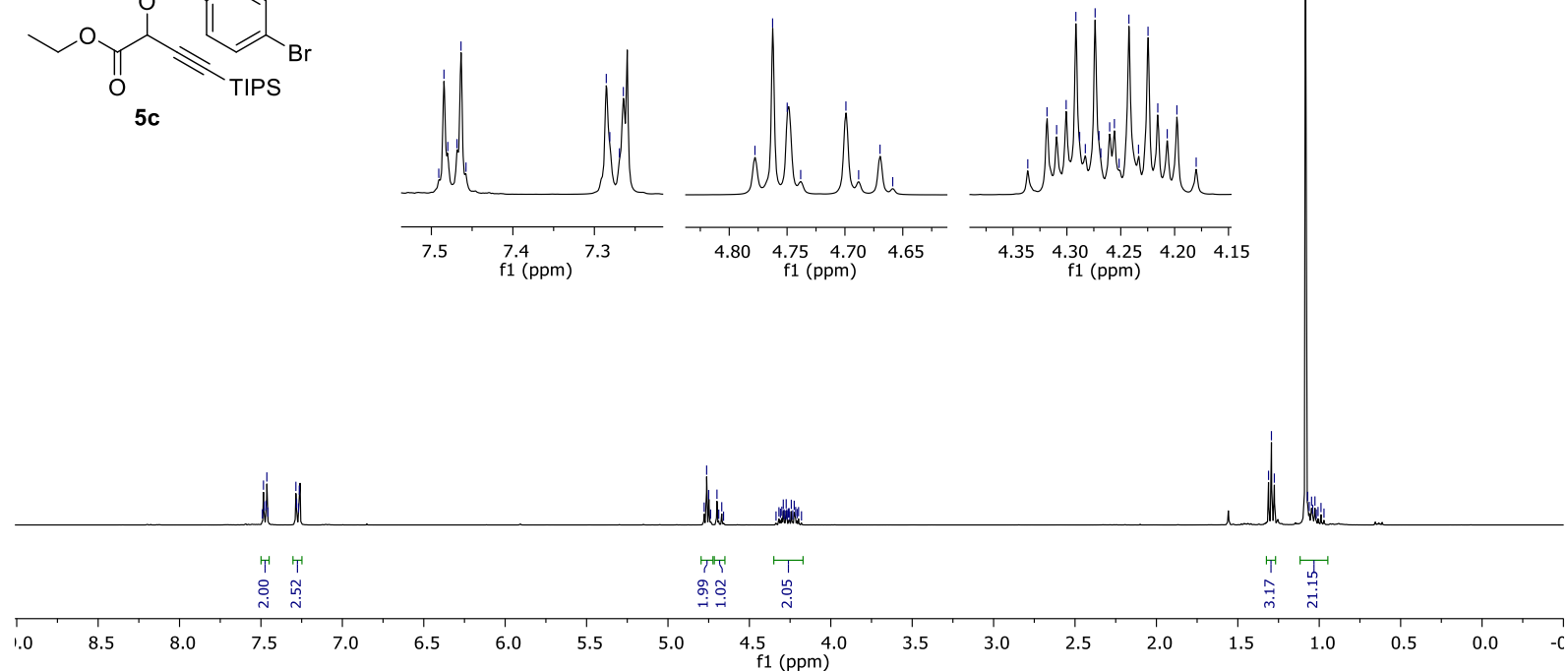

${ }^{13}$ C-NMR (101 MHz, $\left.\mathrm{CDCl}_{3}\right)$ of compound 5c

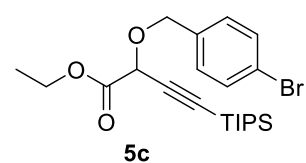

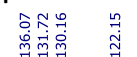


${ }^{1} \mathbf{H}-\mathrm{NMR}\left(400 \mathrm{MHz}, \mathrm{CDCl}_{3}\right)$ of compound $\mathbf{5 d}$

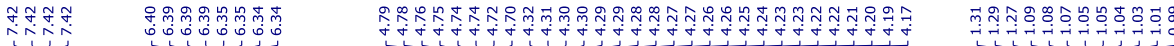
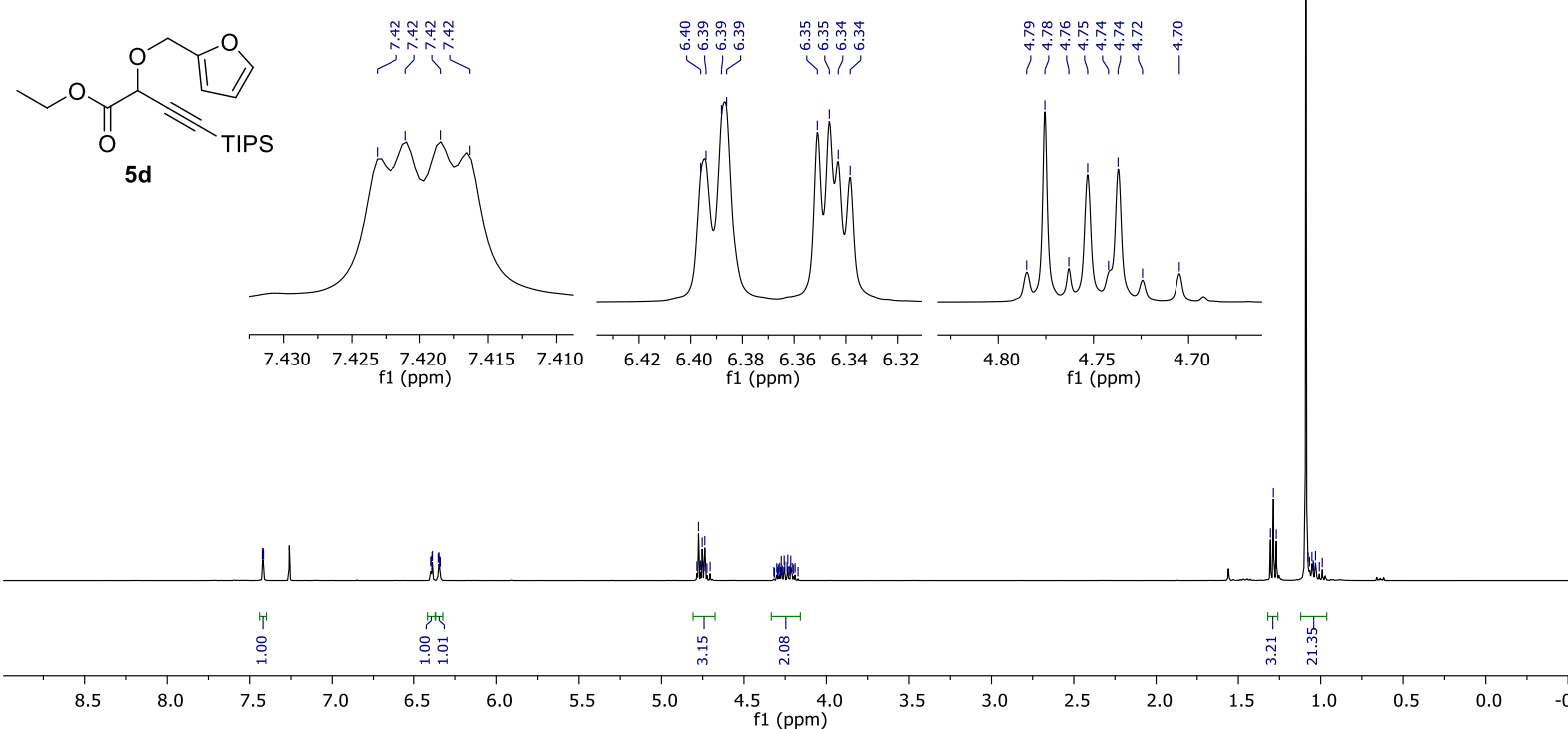

${ }^{13}$ C-NMR (101 MHz, $\left.\mathrm{CDCl}_{3}\right)$ of compound $\mathbf{5 d}$

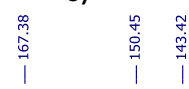

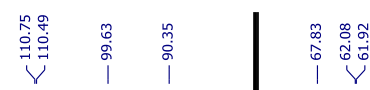

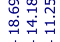
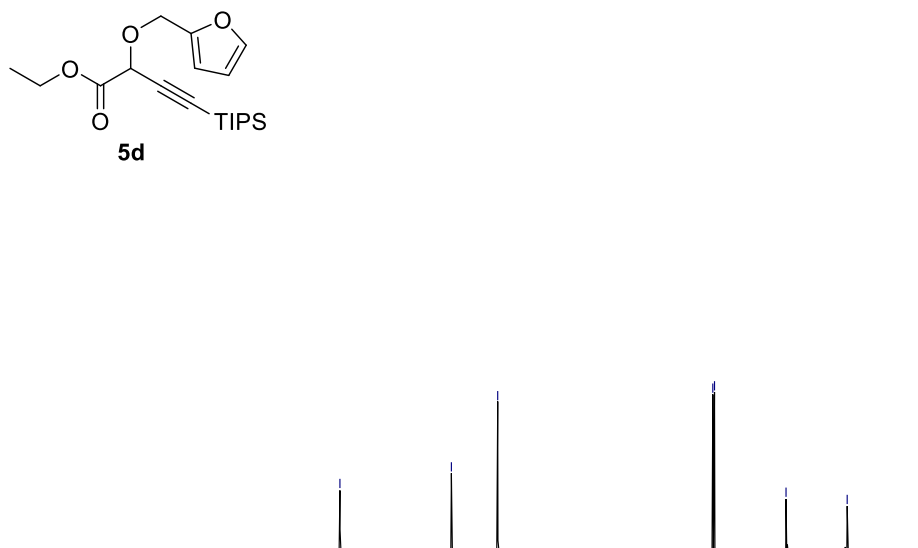

个

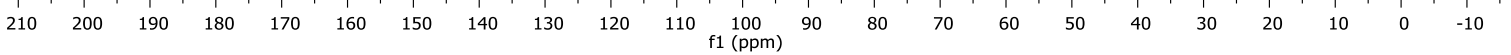


${ }^{1} \mathbf{H}-\mathrm{NMR}\left(400 \mathrm{MHz}, \mathrm{CDCl}_{3}\right)$ of compound $\mathbf{5 e}$

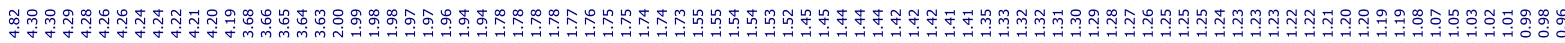
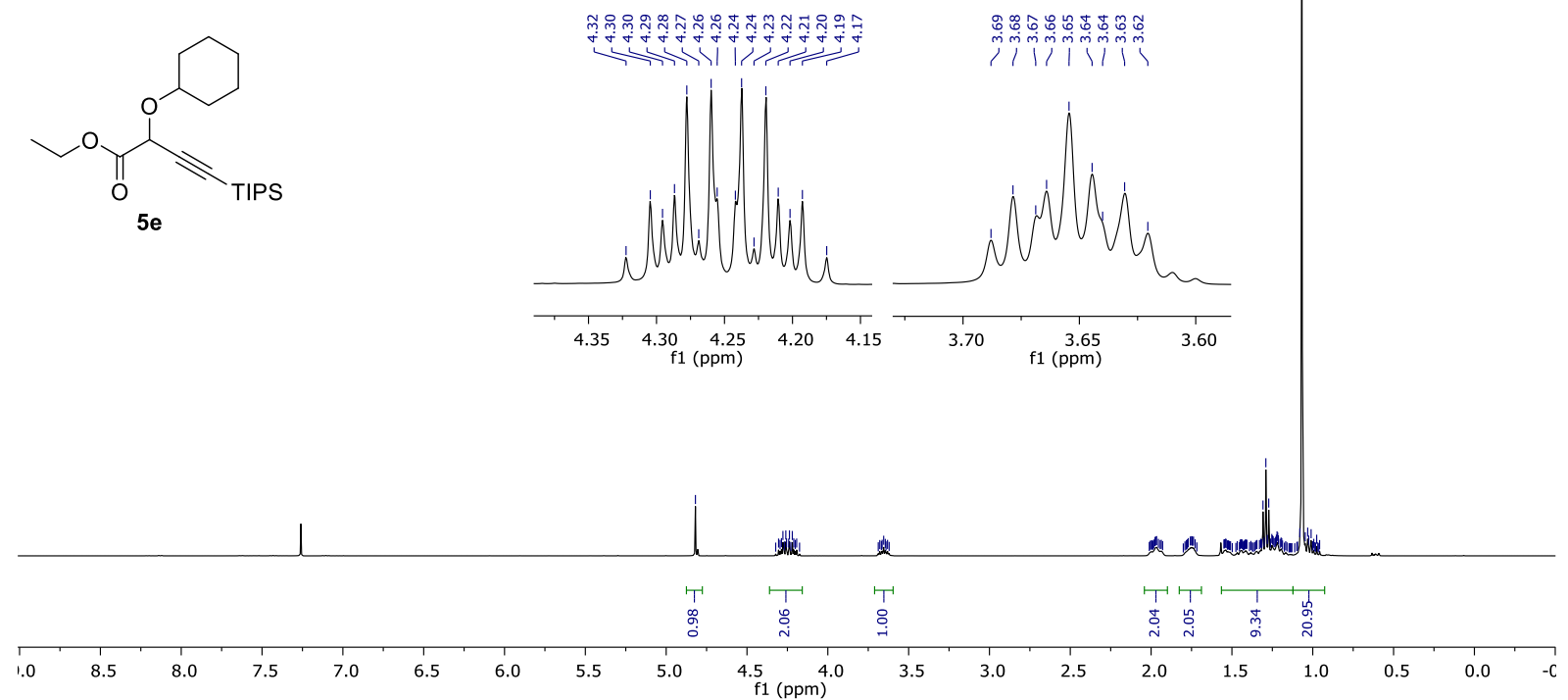

${ }^{13} \mathrm{C}-\mathrm{NMR}\left(101 \mathrm{MHz}, \mathrm{CDCl}_{3}\right)$ of compound $\mathbf{5 e}$

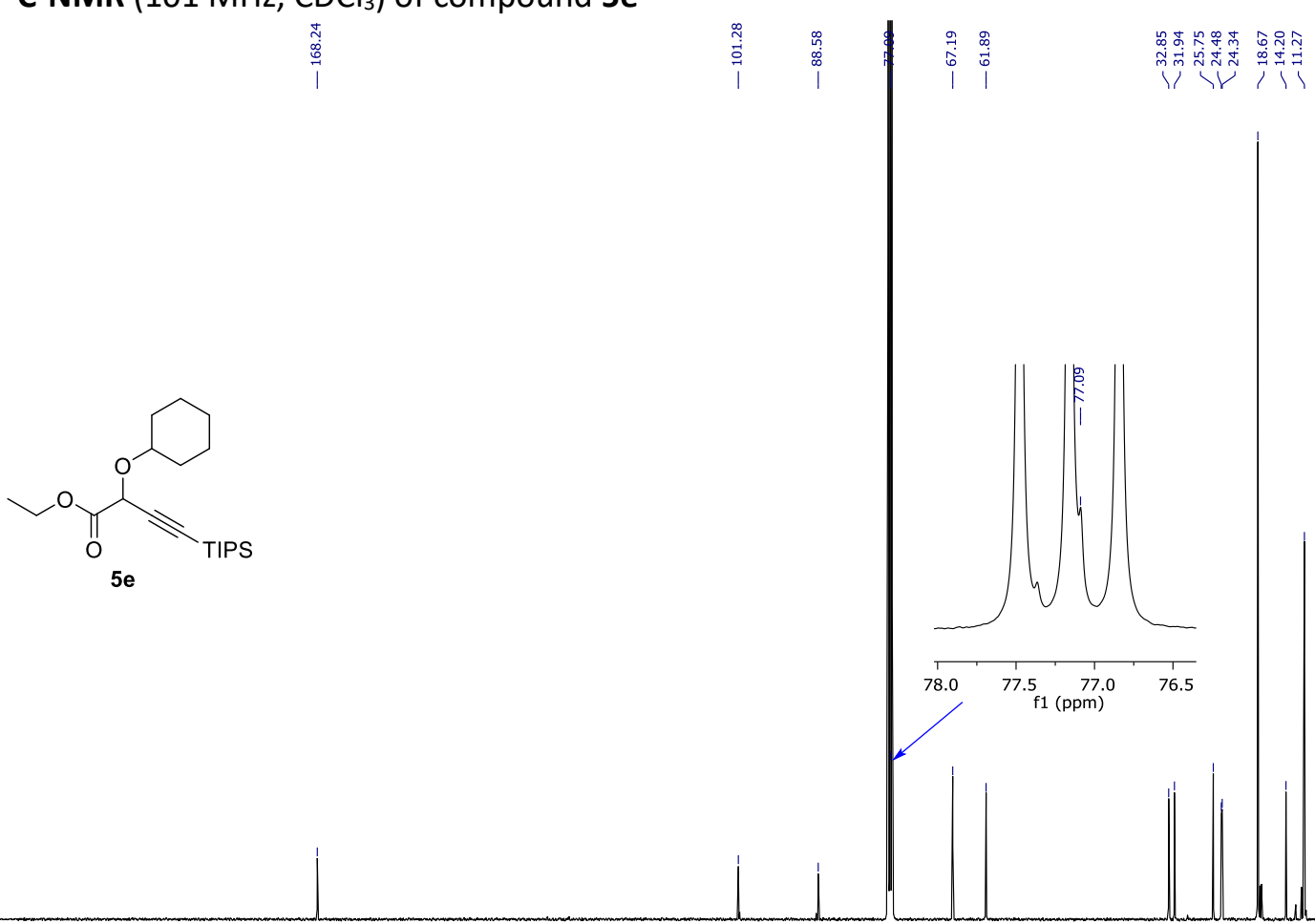

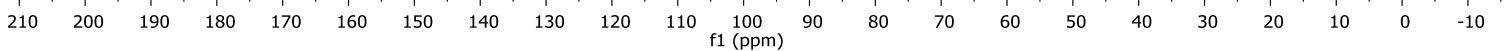


${ }^{1} \mathbf{H}-\mathrm{NMR}\left(400 \mathrm{MHz}, \mathrm{CDCl}_{3}\right)$ of compound $\mathbf{5 f}$

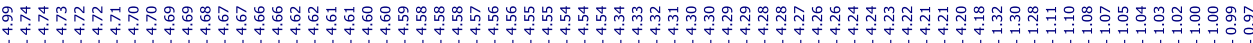
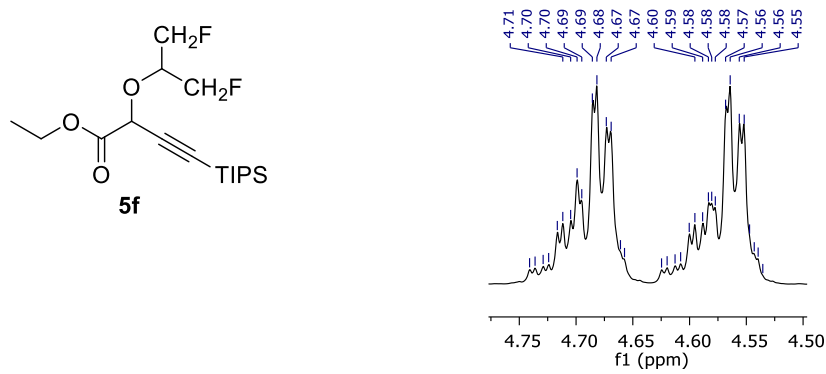

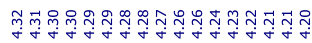
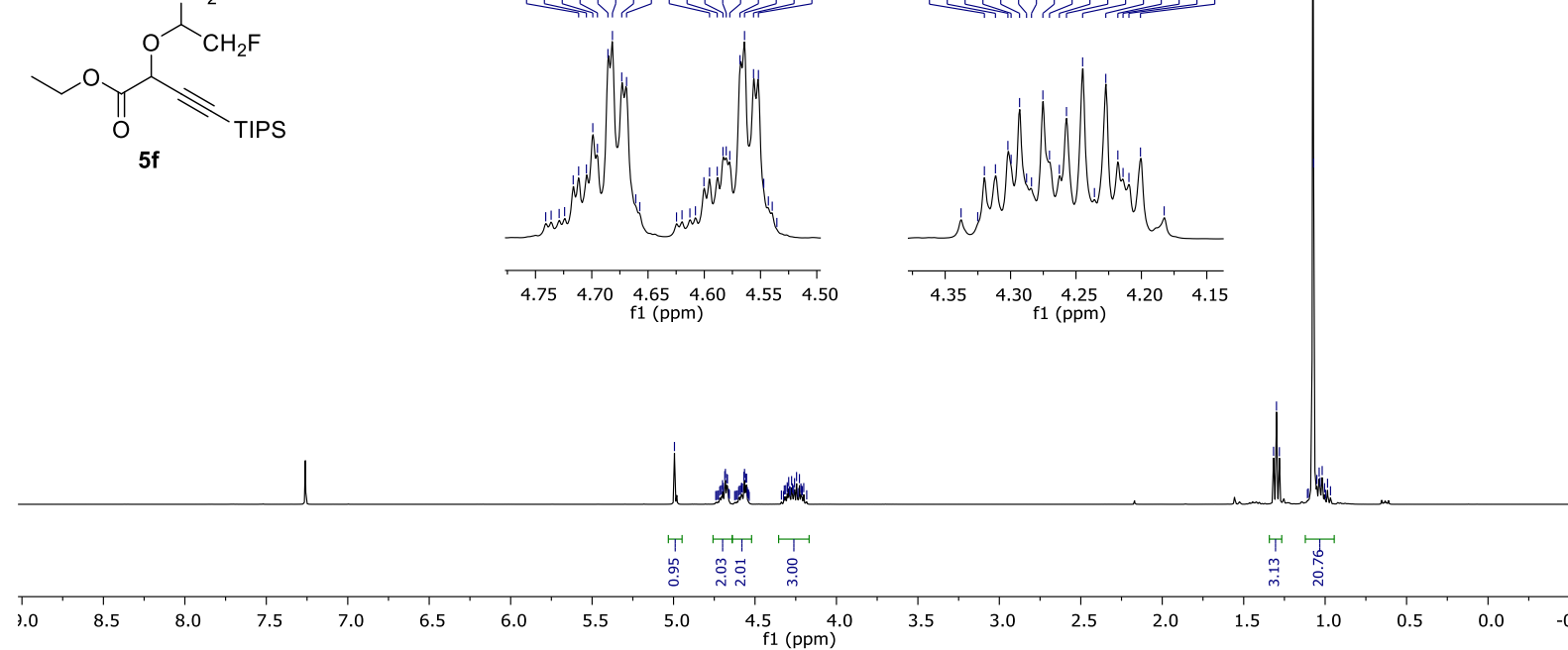

${ }^{13} \mathrm{C}-\mathrm{NMR}\left(101 \mathrm{MHz}, \mathrm{CDCl}_{3}\right)$ of compound $\mathbf{5 f}$

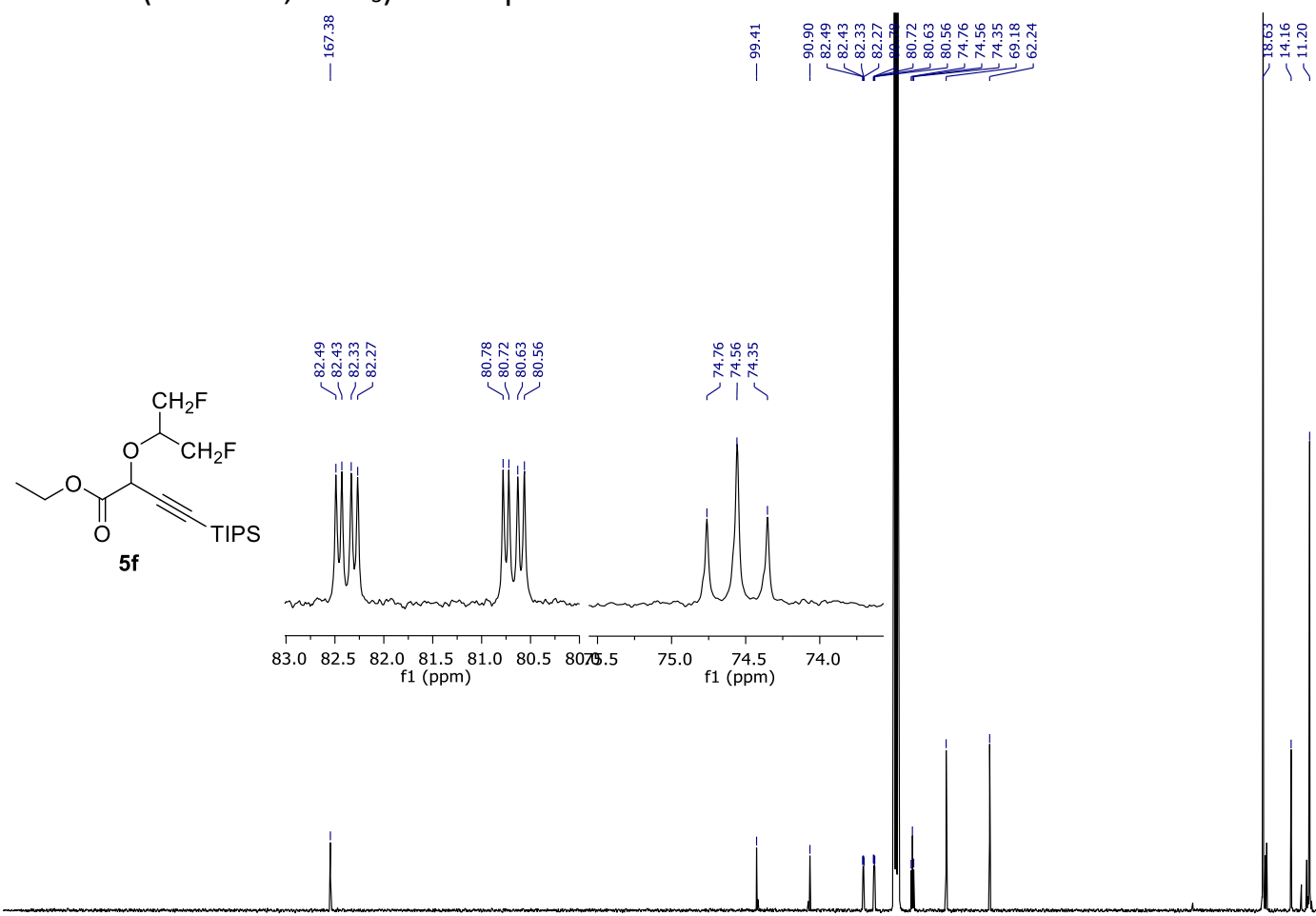

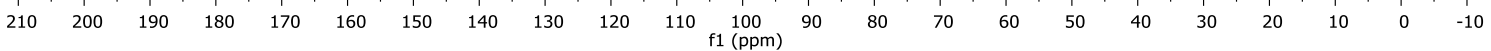


${ }^{19}$ F-NMR (376 MHz, $\mathrm{CDCl}_{3}$ ) of compound $\mathbf{5 f}$

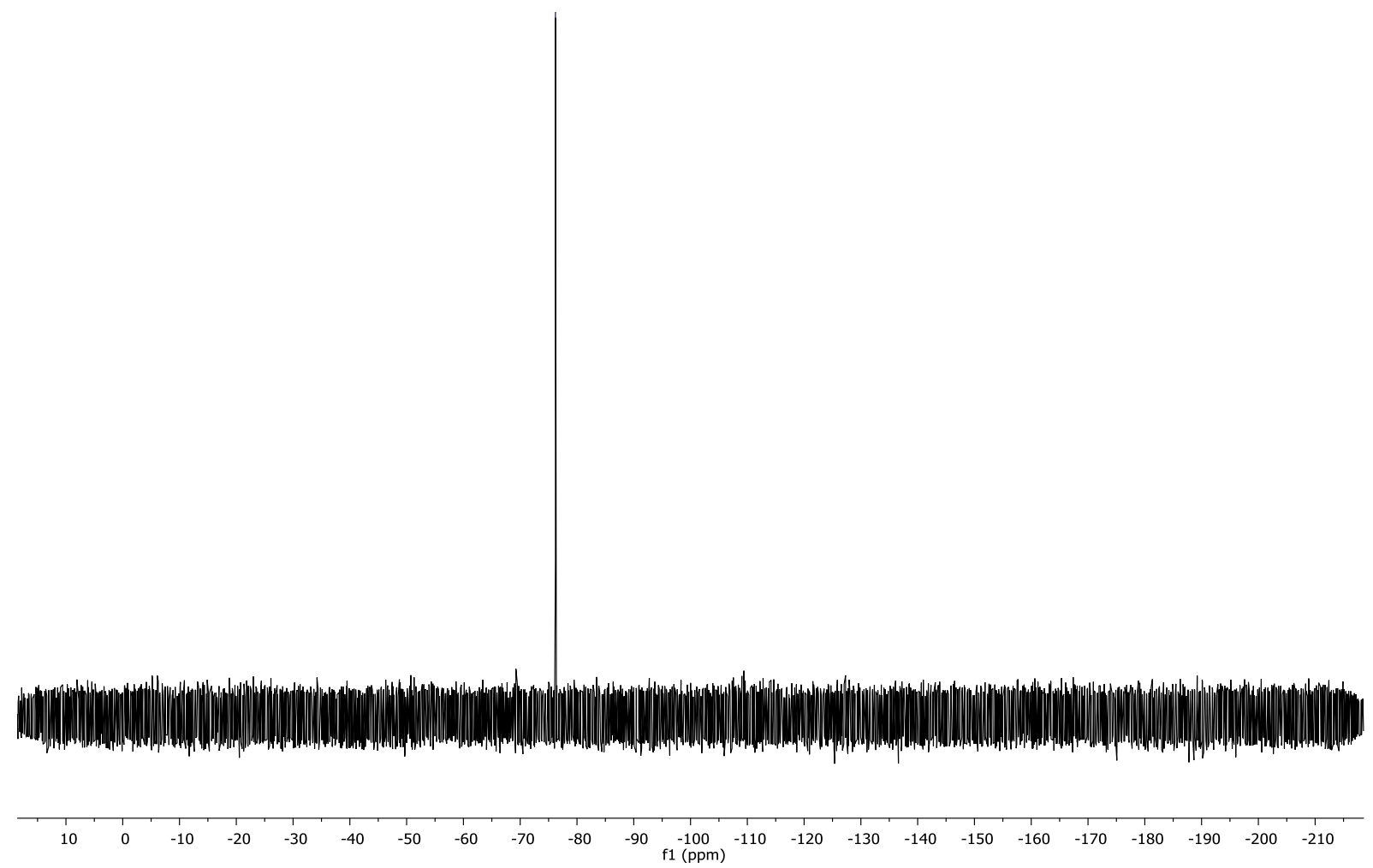




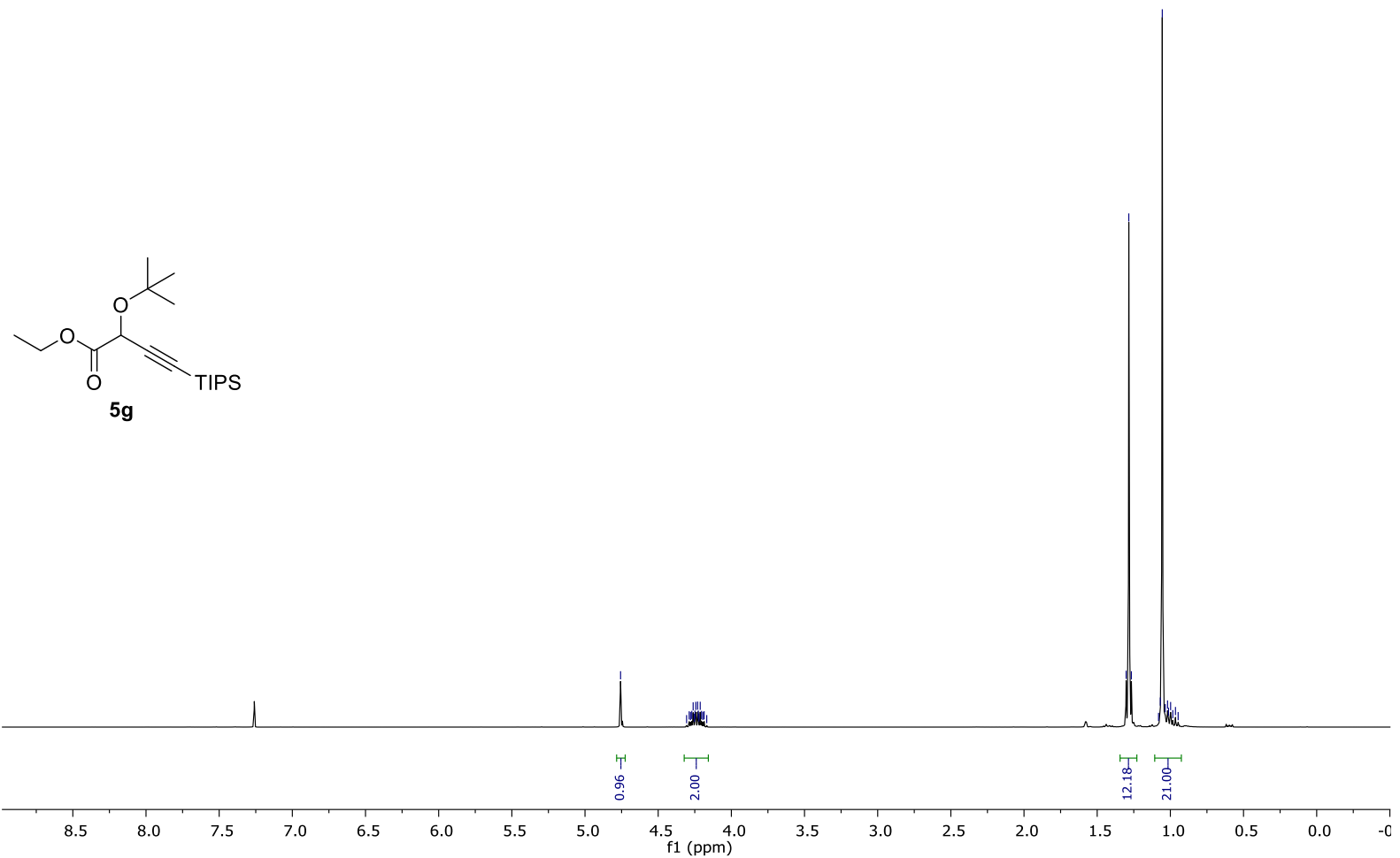

${ }^{13} \mathrm{C}-\mathrm{NMR}\left(101 \mathrm{MHz}, \mathrm{CDCl}_{3}\right)$ of compound $\mathbf{5 g}$

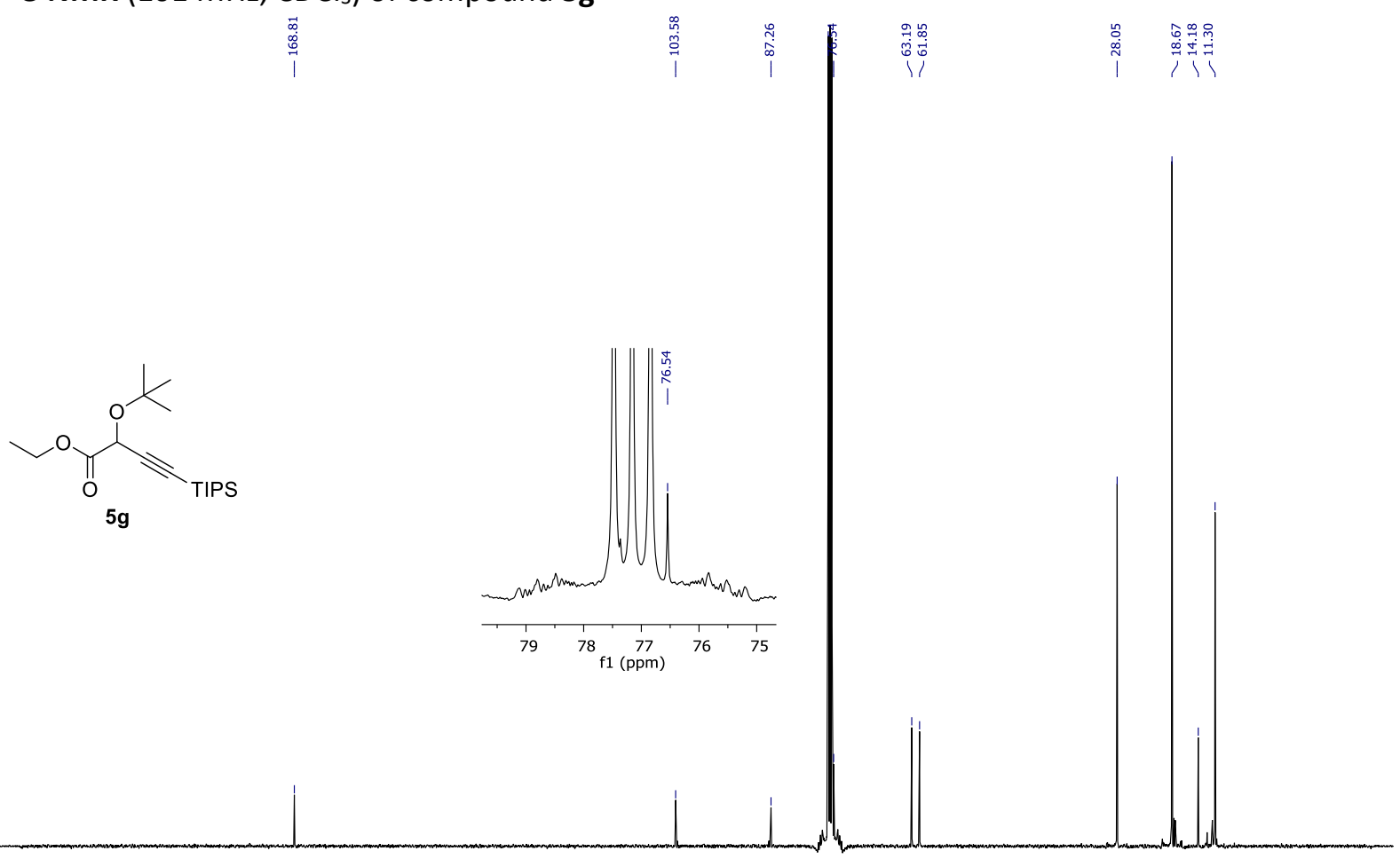

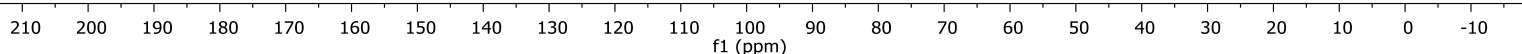


${ }^{1} \mathbf{H}-\mathrm{NMR}\left(400 \mathrm{MHz}, \mathrm{CDCl}_{3}\right)$ of compound $\mathbf{5 h}$

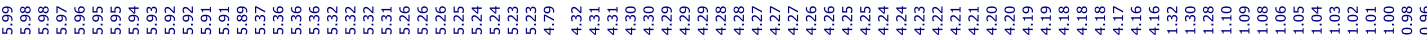
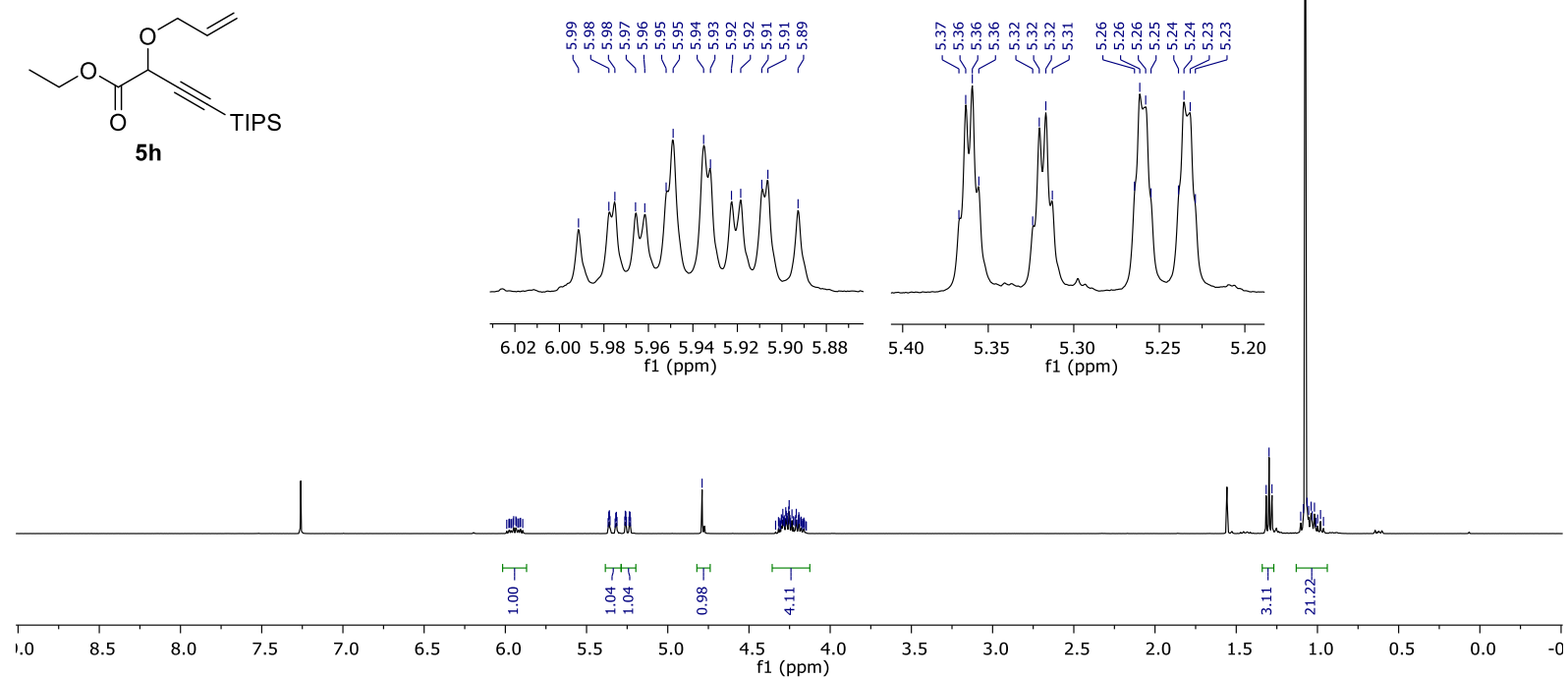

${ }^{13} \mathrm{C}-\mathrm{NMR}\left(101 \mathrm{MHz}, \mathrm{CDCl}_{3}\right)$ of compound $\mathbf{5 h}$

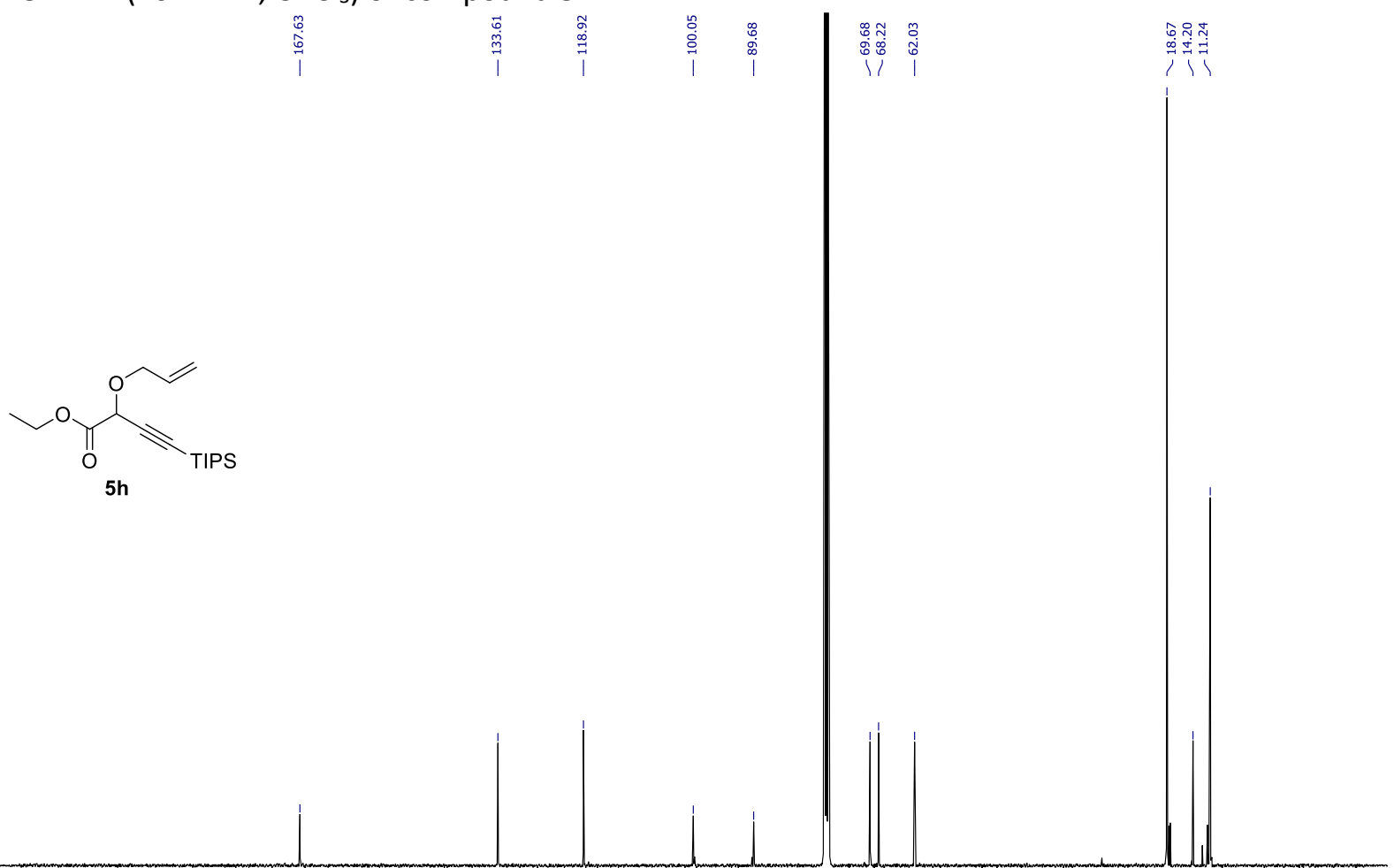

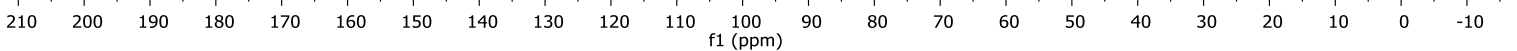


${ }^{1} \mathbf{H}-\mathrm{NMR}\left(400 \mathrm{MHz}, \mathrm{CDCl}_{3}\right)$ of compound $\mathbf{5 i}$

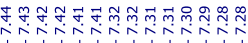

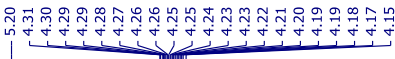

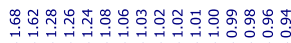

जो
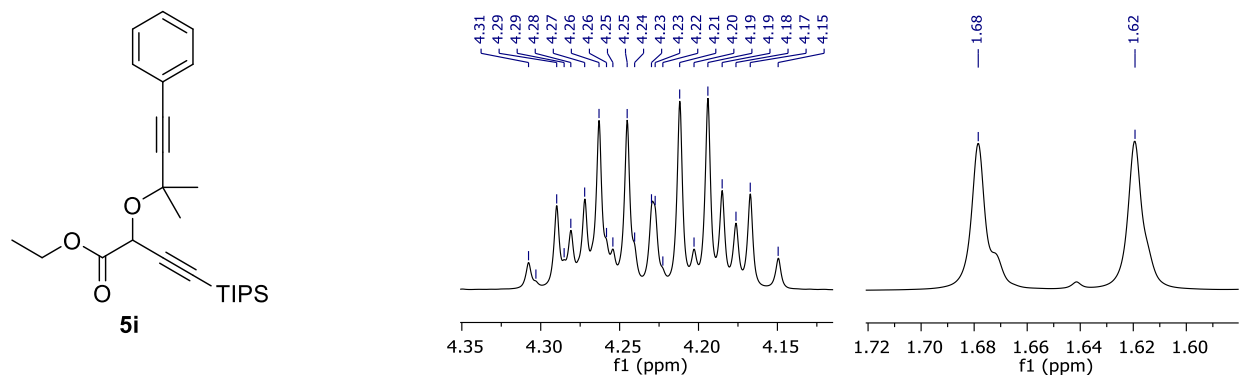

$\begin{array}{lllllllllllll}4.35 & 4.30 & 4.25 & 4.20 & 4.15 & & 1.72 & 1.70 & 1.68 & 1.66 & 1.64 & 1.62 & 1.60 \\ \mathrm{f} 1(\mathrm{ppm})\end{array}$

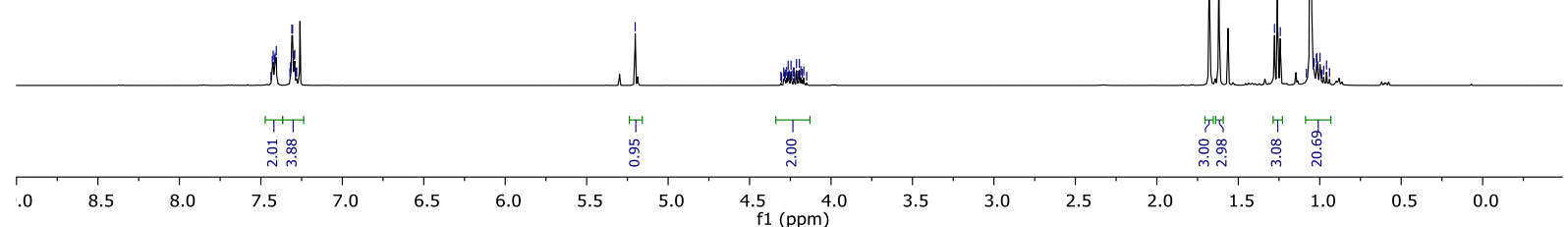

${ }^{13}$ C-NMR (101 MHz, $\mathrm{CDCl}_{3}$ ) of compound $\mathbf{5 i}$

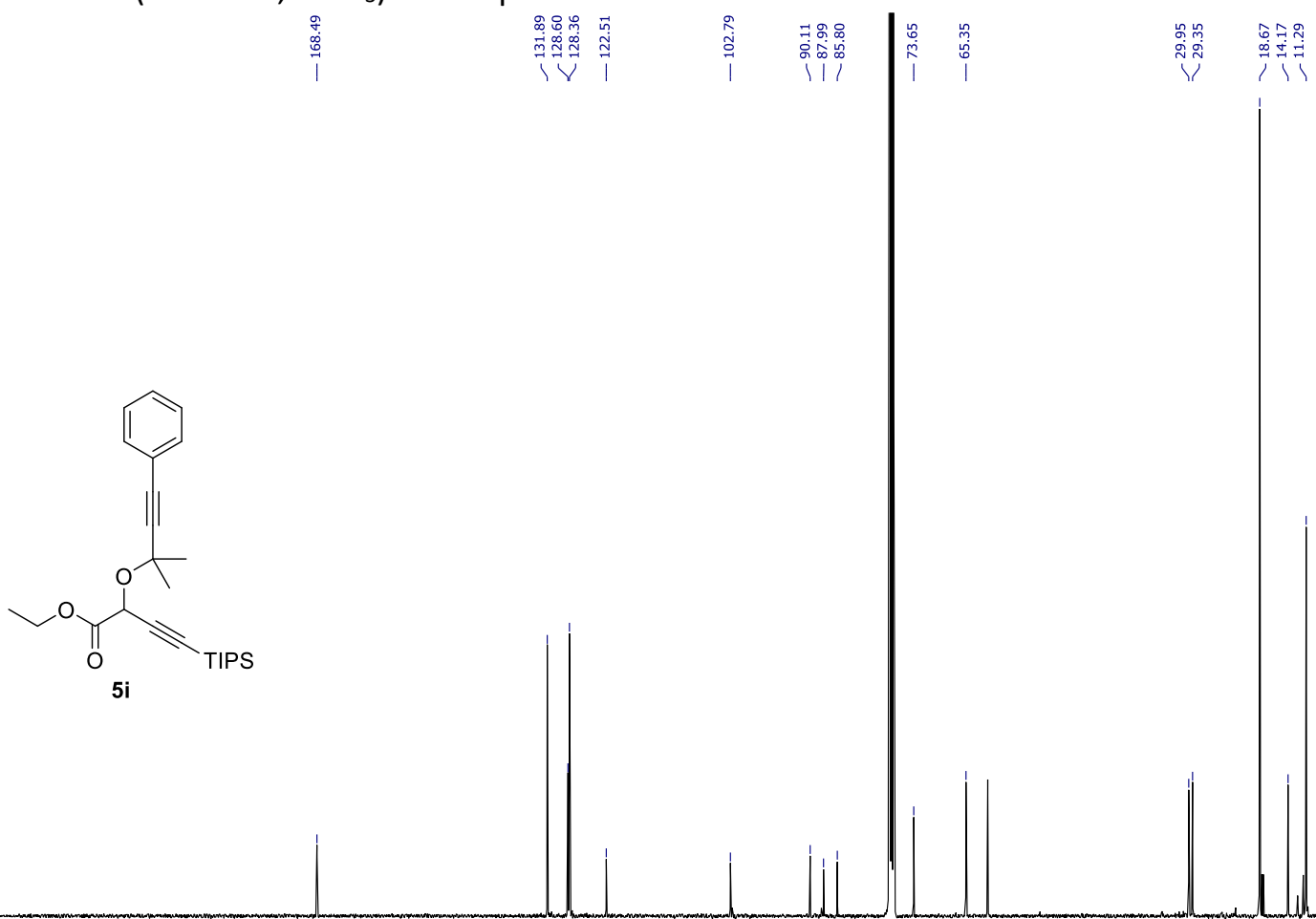

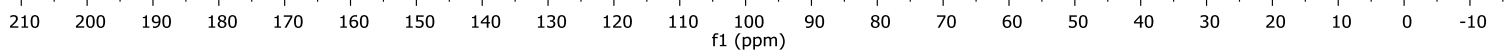


${ }^{1} \mathbf{H}-\mathrm{NMR}\left(400 \mathrm{MHz}, \mathrm{CDCl}_{3}\right)$ of compound $\mathbf{5 j}$
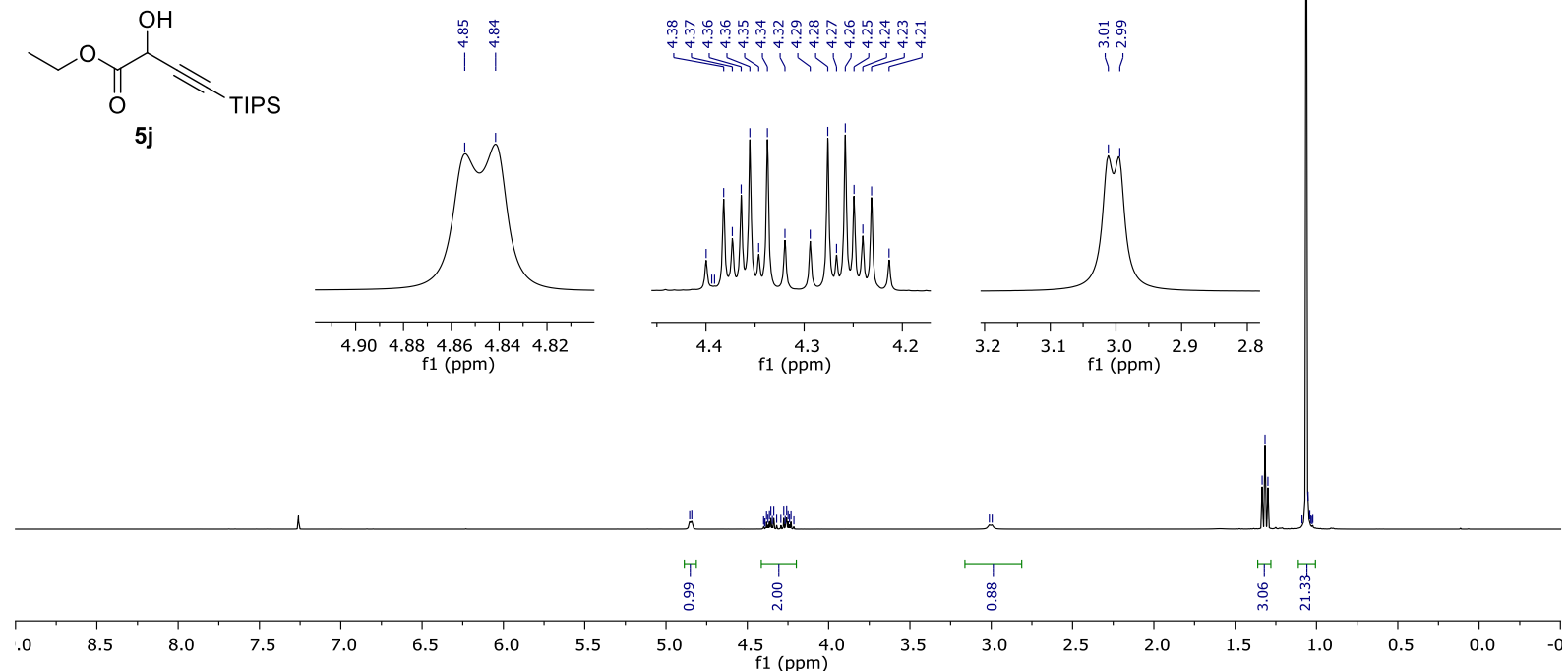

${ }^{13}$ C-NMR (101 MHz, $\mathrm{CDCl}_{3}$ ) of compound $\mathbf{5 j}$ 乫

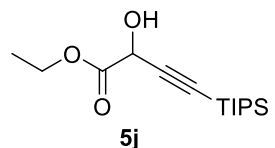




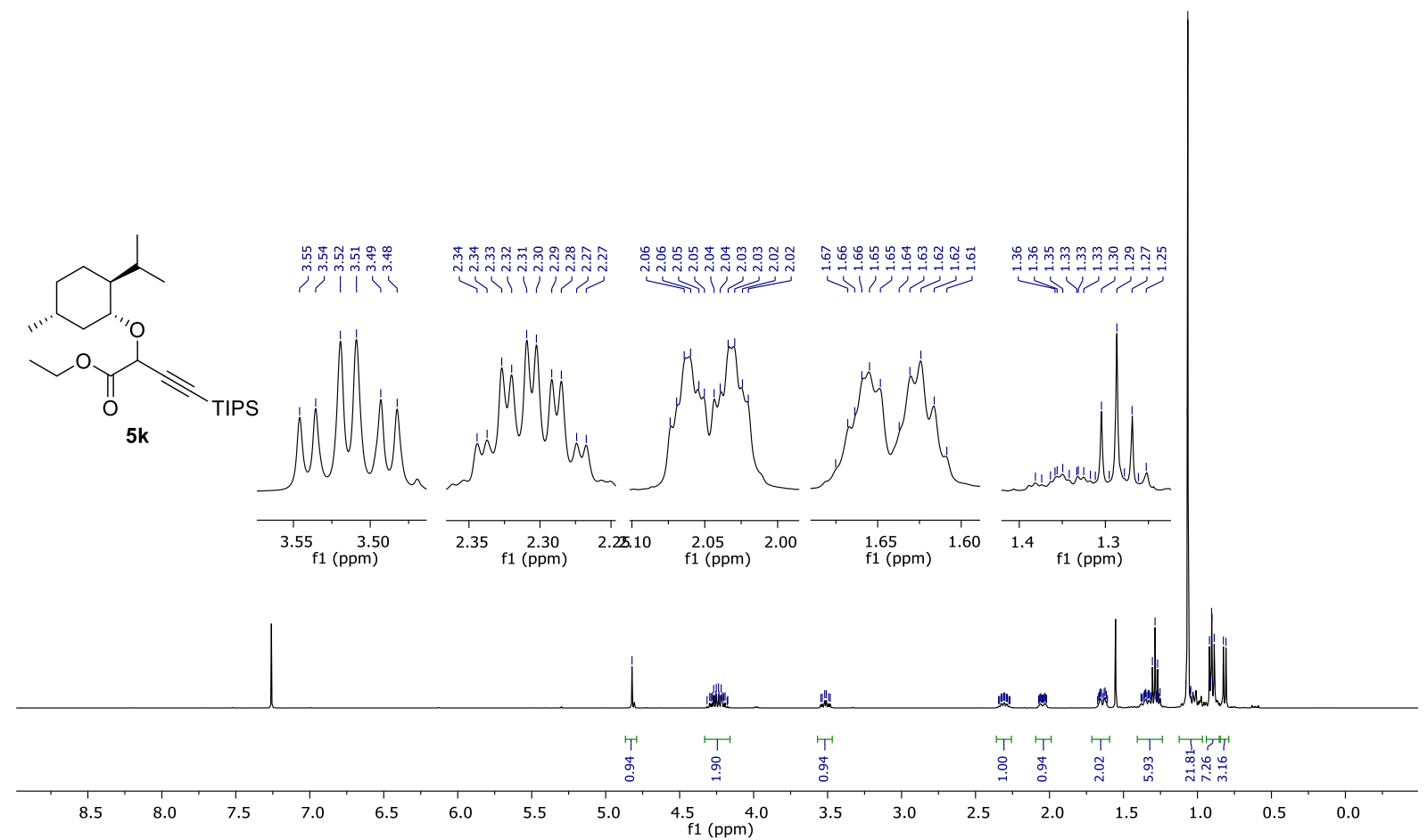

${ }^{13} \mathbf{C}-\mathrm{NMR}\left(101 \mathrm{MHz}, \mathrm{CDCl}_{3}\right.$ ) of compound $\mathbf{5 k}$ (first diastereoisomer)

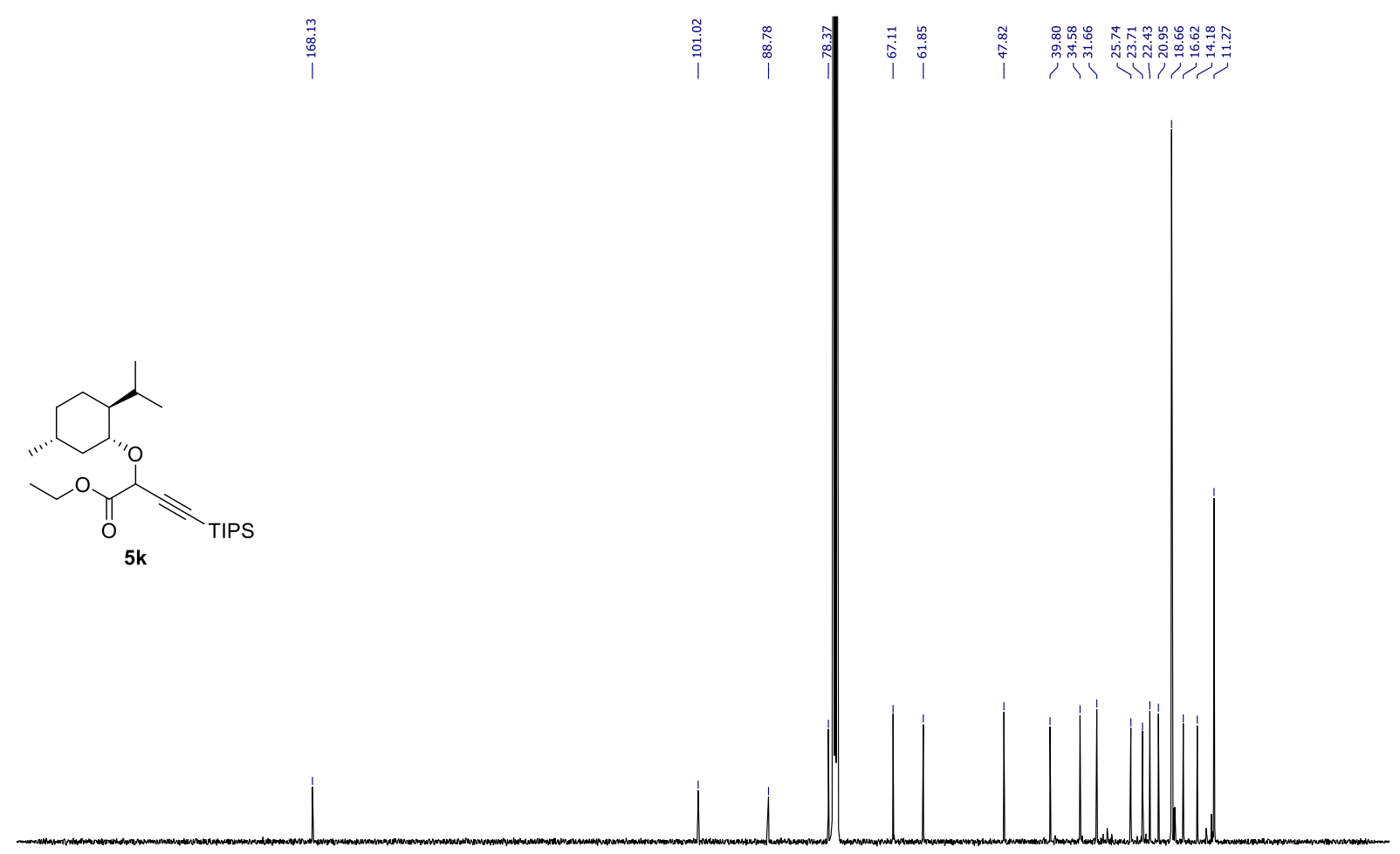

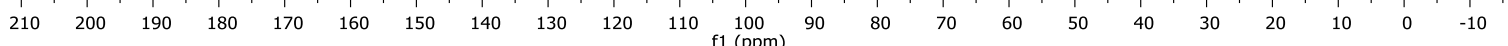




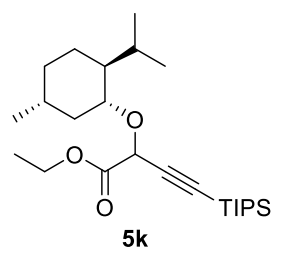

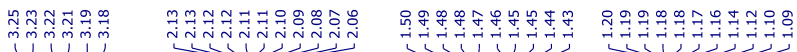
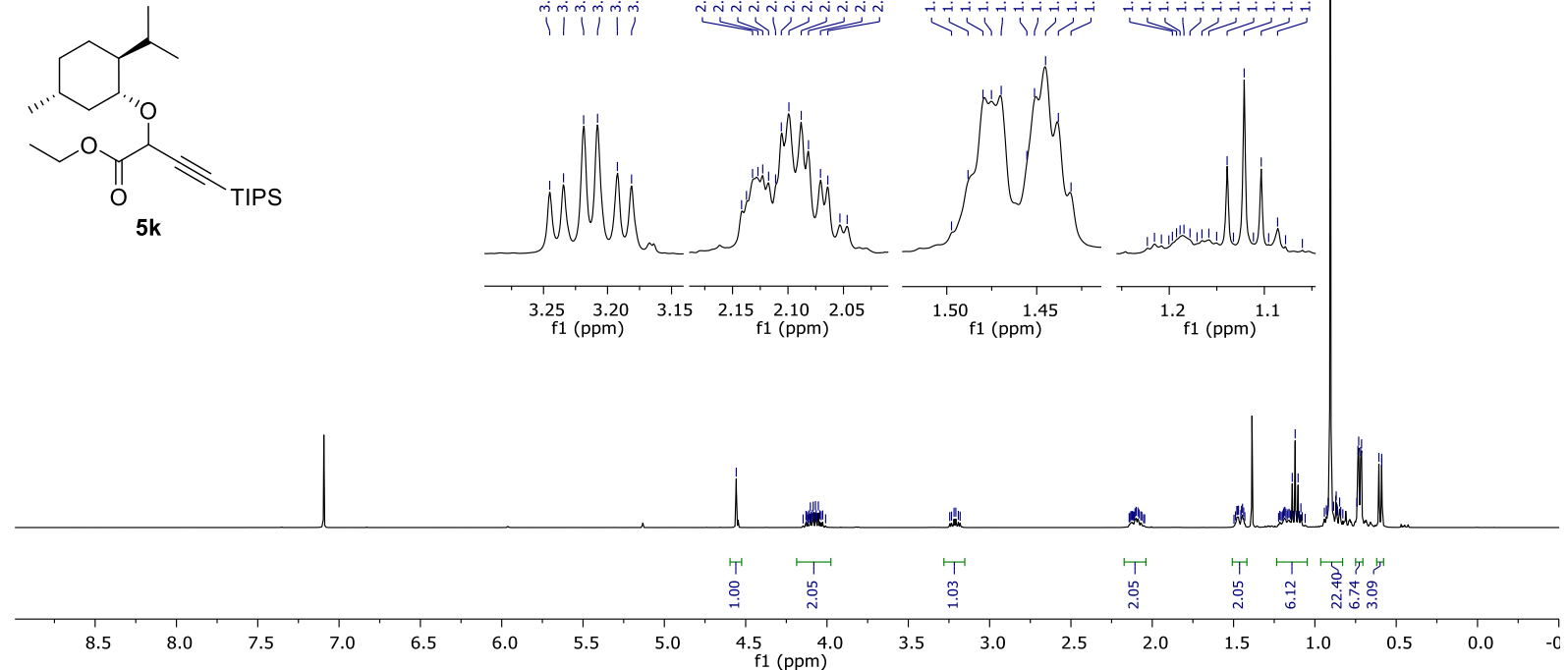

${ }^{13} \mathrm{C}-\mathrm{NMR}\left(101 \mathrm{MHz}, \mathrm{CDCl}_{3}\right)$ of compound $\mathbf{5 k}$ (second diastereoisomer)

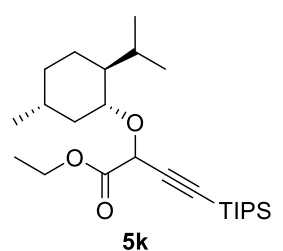

$5 \mathbf{k}$

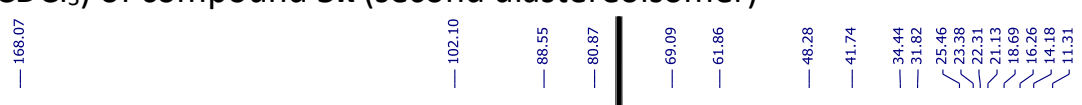

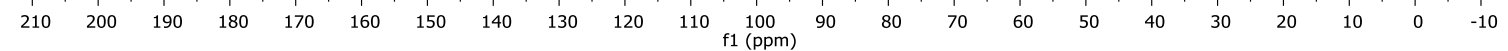


${ }^{1}$ H-NMR $\left(400 \mathrm{MHz}, \mathrm{CDCl}_{3}\right)$ of compound $5 \mathbf{I}$

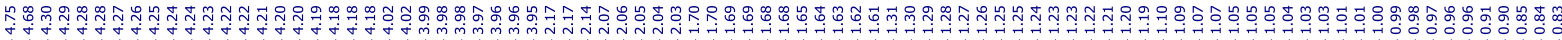
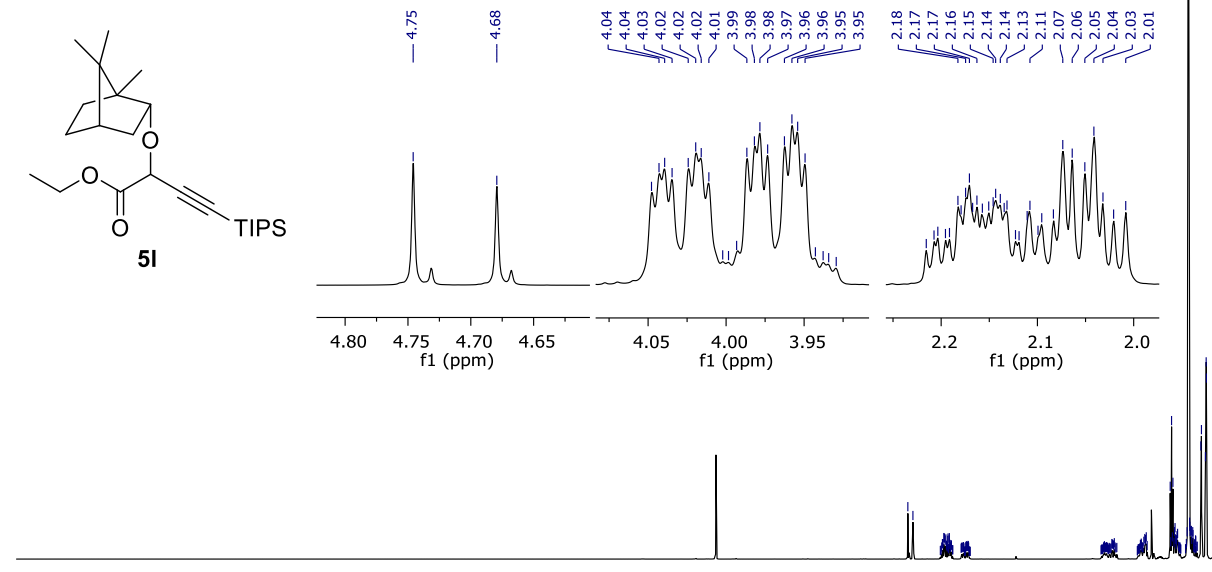

Tी श't

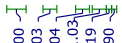

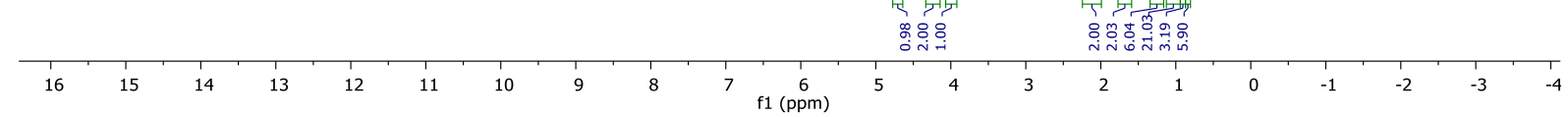

${ }^{13}$ C-NMR (101 MHz, $\left.\mathrm{CDCl}_{3}\right)$ of compound 5 I

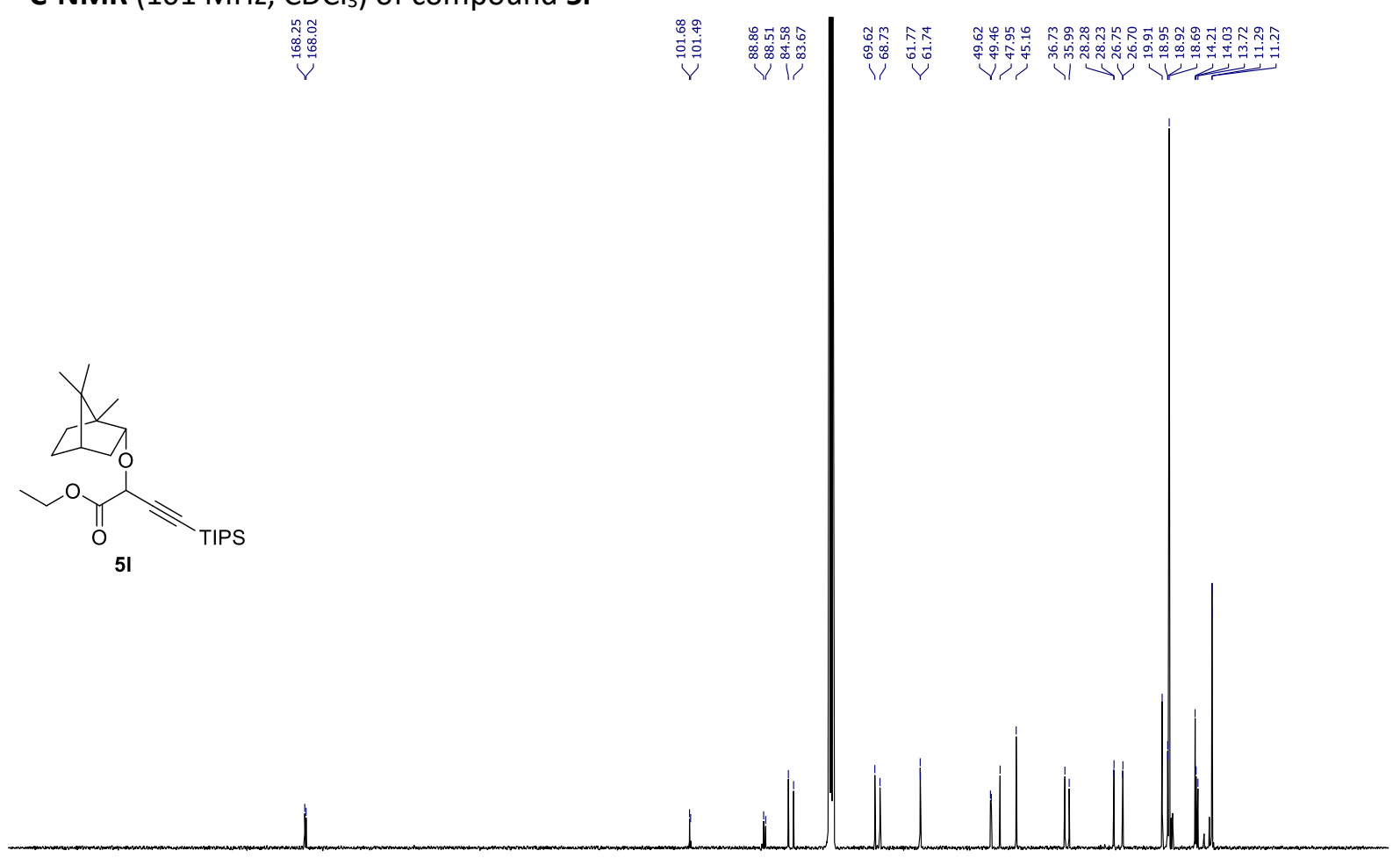

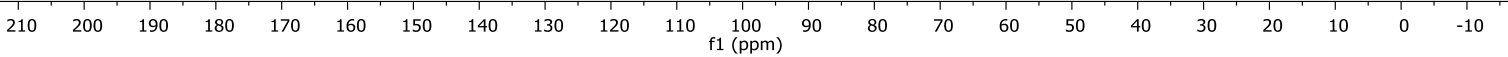



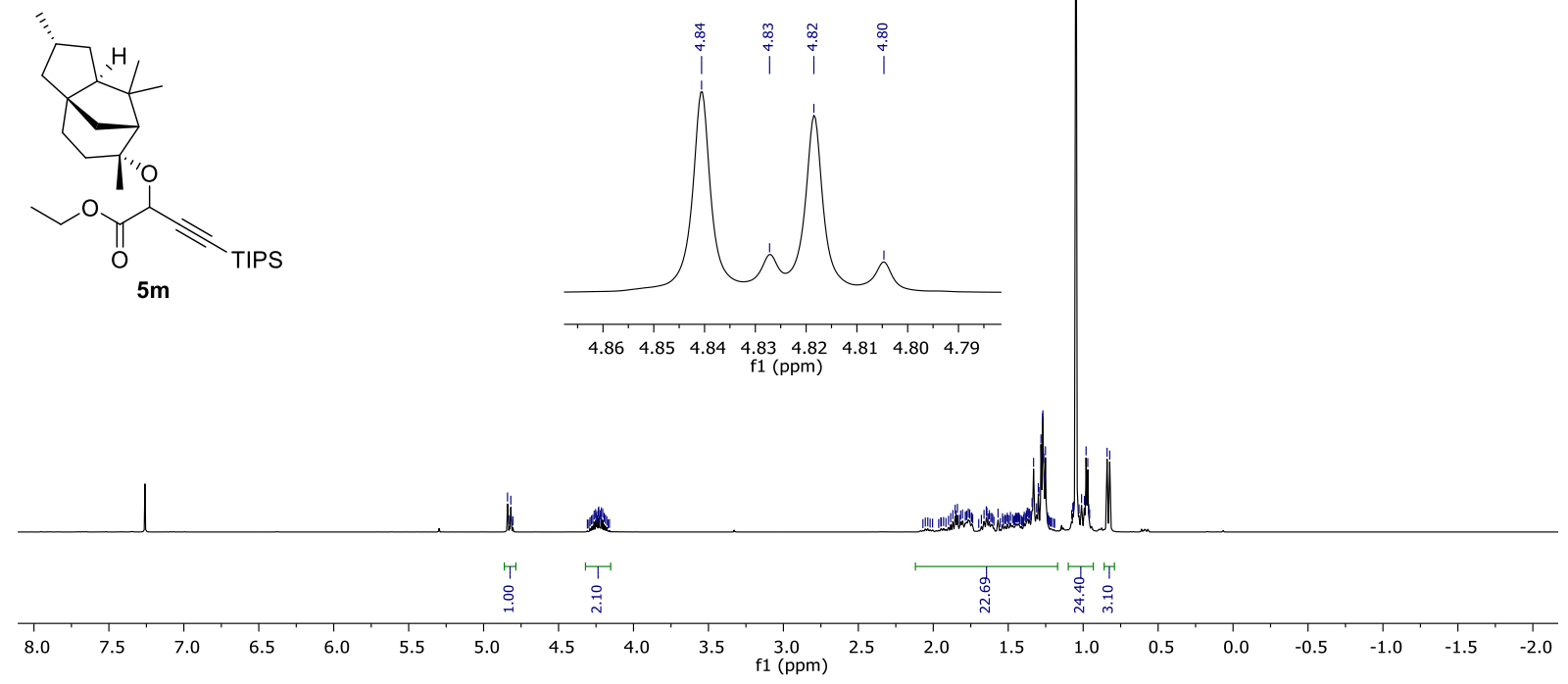

${ }^{13} \mathrm{C}-\mathrm{NMR}\left(101 \mathrm{MHz}, \mathrm{CDCl}_{3}\right)$ of compound $\mathbf{5 m}$ 产密
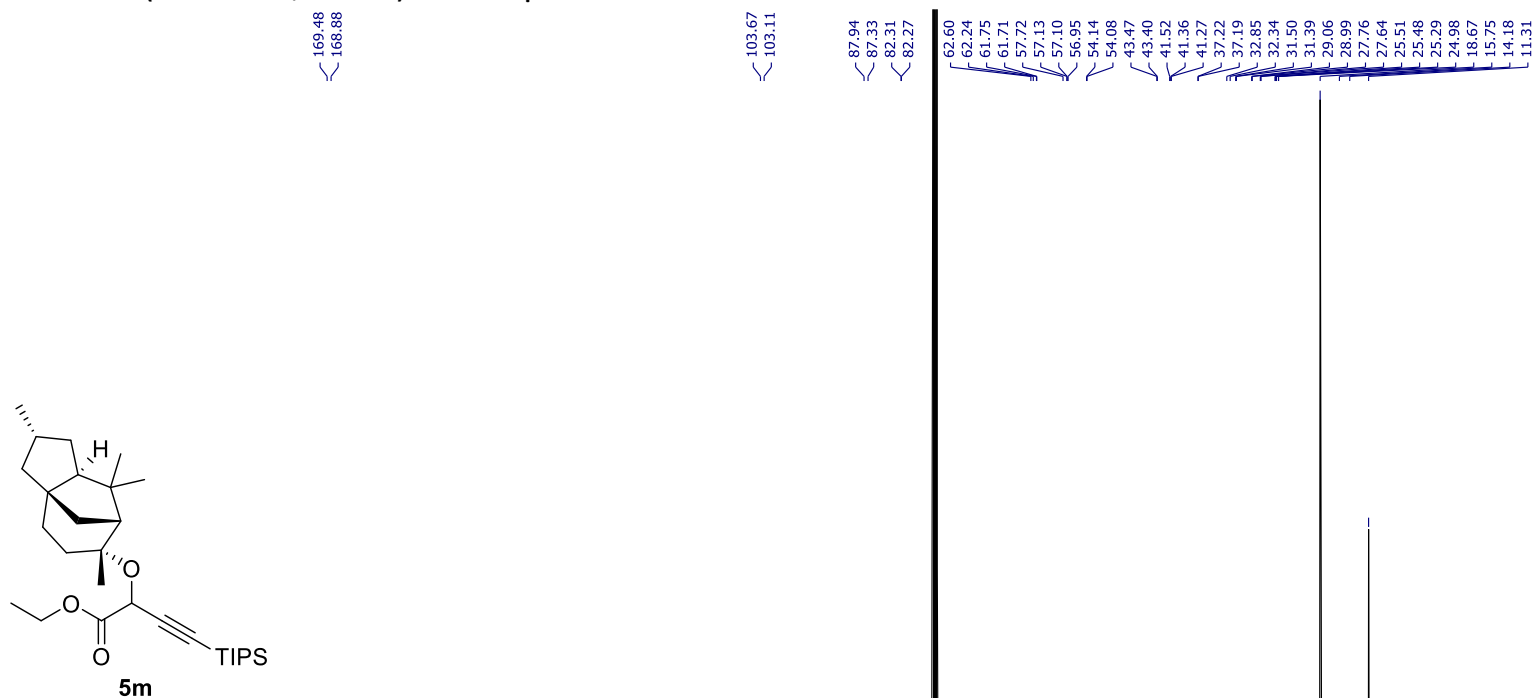

$5 \mathrm{~m}$ 
${ }^{1} \mathbf{H}-\mathrm{NMR}\left(400 \mathrm{MHz}, \mathrm{CDCl}_{3}\right)$ of compound $\mathbf{5 n}$

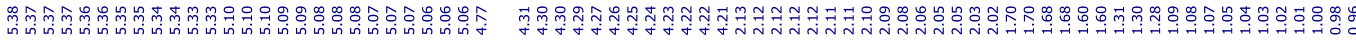

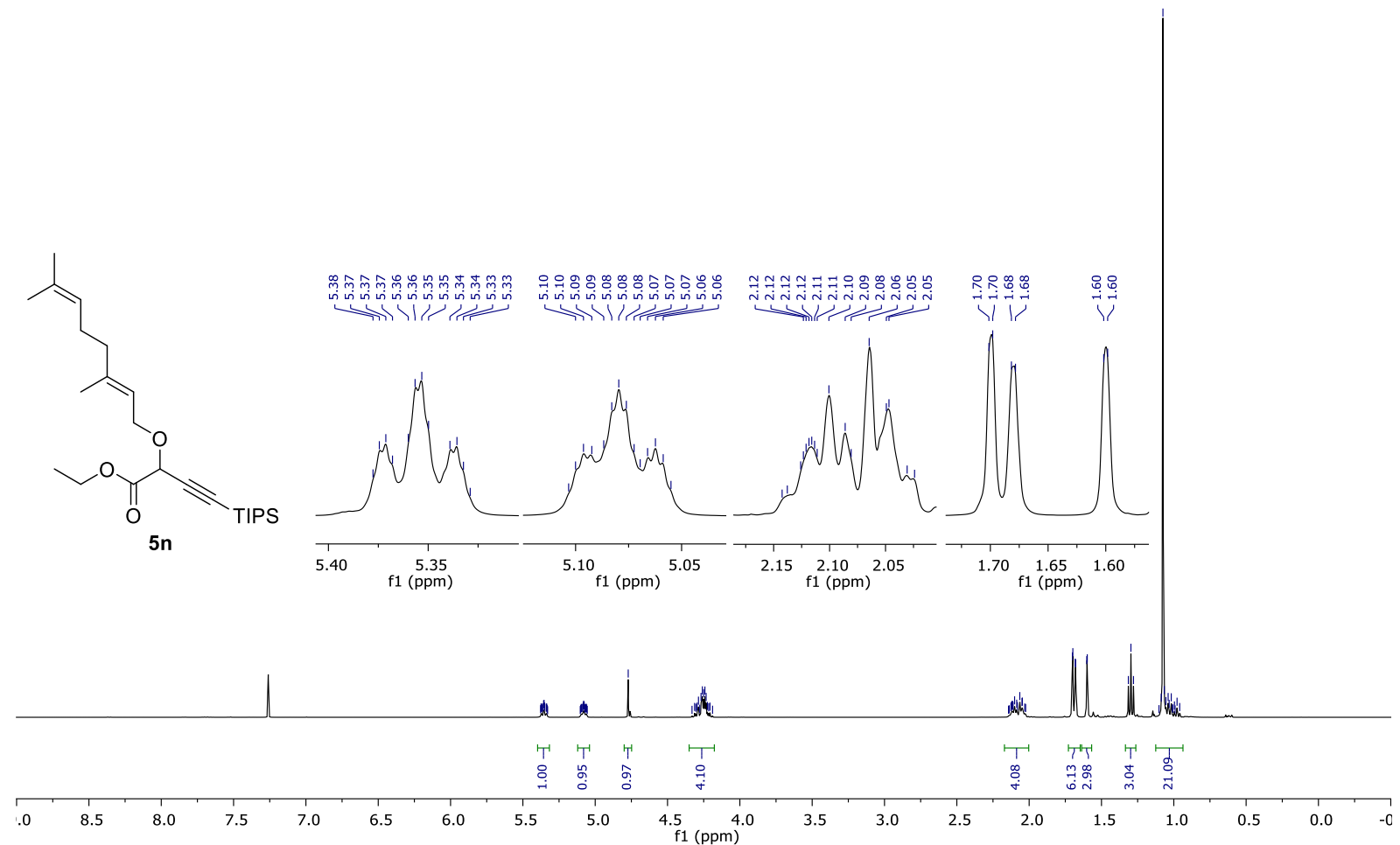

${ }^{13} \mathrm{C}-\mathrm{NMR}\left(101 \mathrm{MHz}, \mathrm{CDCl}_{3}\right)$ of compound $\mathbf{5 n}$

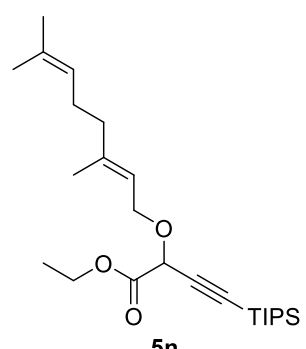

$5 n$

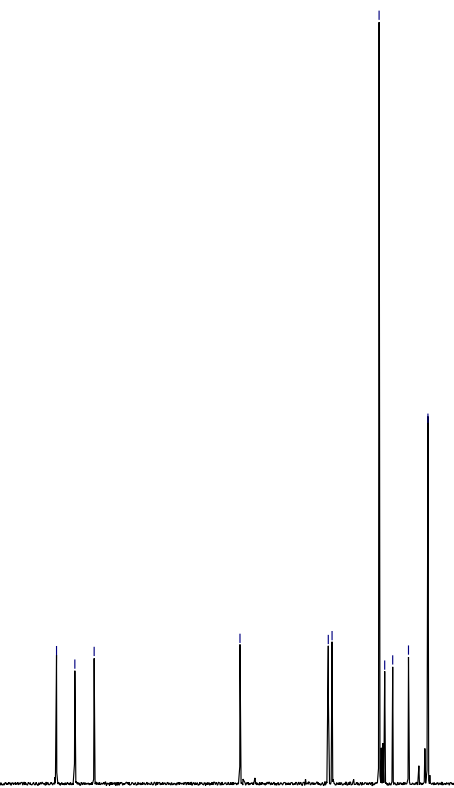

$\begin{array}{lllllllllll}210 & 200 & 190 & 180 & 170 & 160 & 150 & 140 & 130 & 120 & 110 \begin{array}{c}100 \\ \mathrm{f} 1(\mathrm{ppm})\end{array}\end{array}$ 
${ }^{1} \mathrm{H}-\mathrm{NMR}\left(400 \mathrm{MHz}, \mathrm{CDCl}_{3}\right)$ of compound 50

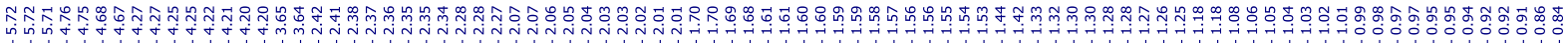
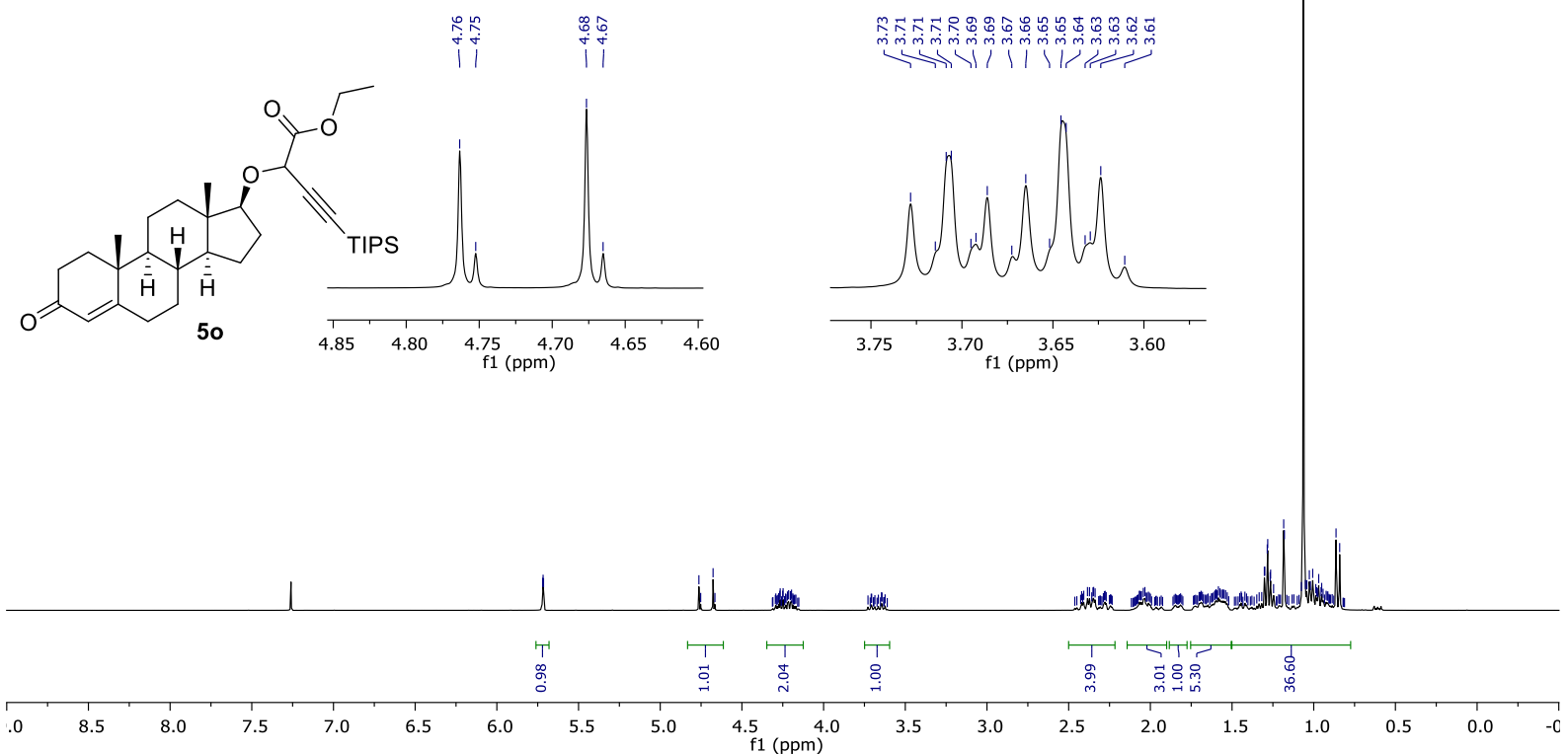

${ }^{13}$ C-NMR (101 MHz, $\mathrm{CDCl}_{3}$ ) of compound 50

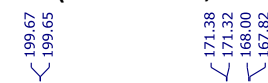

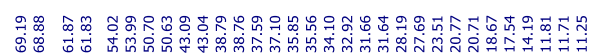

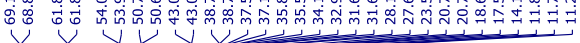<smiles>CC#CC(OC1CCC2C3CC4=CC(=O)CCC4(C)C3CCC12C)C(=O)OCC</smiles>

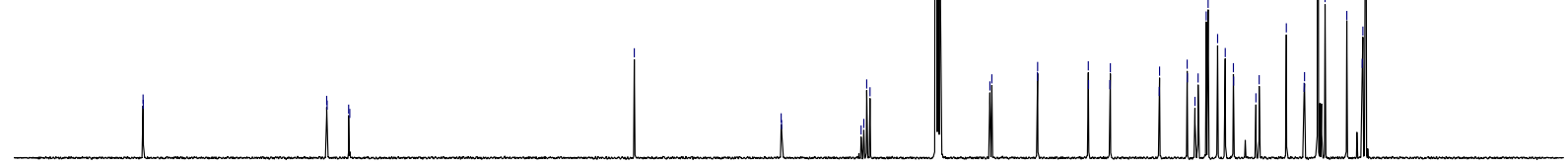

$\begin{array}{llllllllllllllllllllllllll}210 & 200 & 190 & 180 & 170 & 160 & 150 & 140 & 130 & 120 & 110 & \begin{array}{c}100 \\ \mathrm{f} 1(\mathrm{ppm})\end{array} & 90 & 80 & 70 & 60 & 50 & 40 & 30 & 20 & 10 & 0 & -10\end{array}$ 
${ }^{1} \mathrm{H}-\mathrm{NMR}\left(400 \mathrm{MHz}, \mathrm{CDCl}_{3}\right)$ of compound $\mathbf{5 p}$

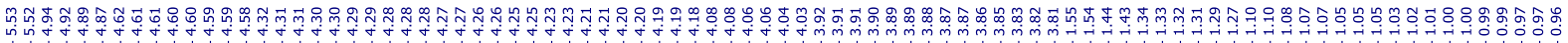
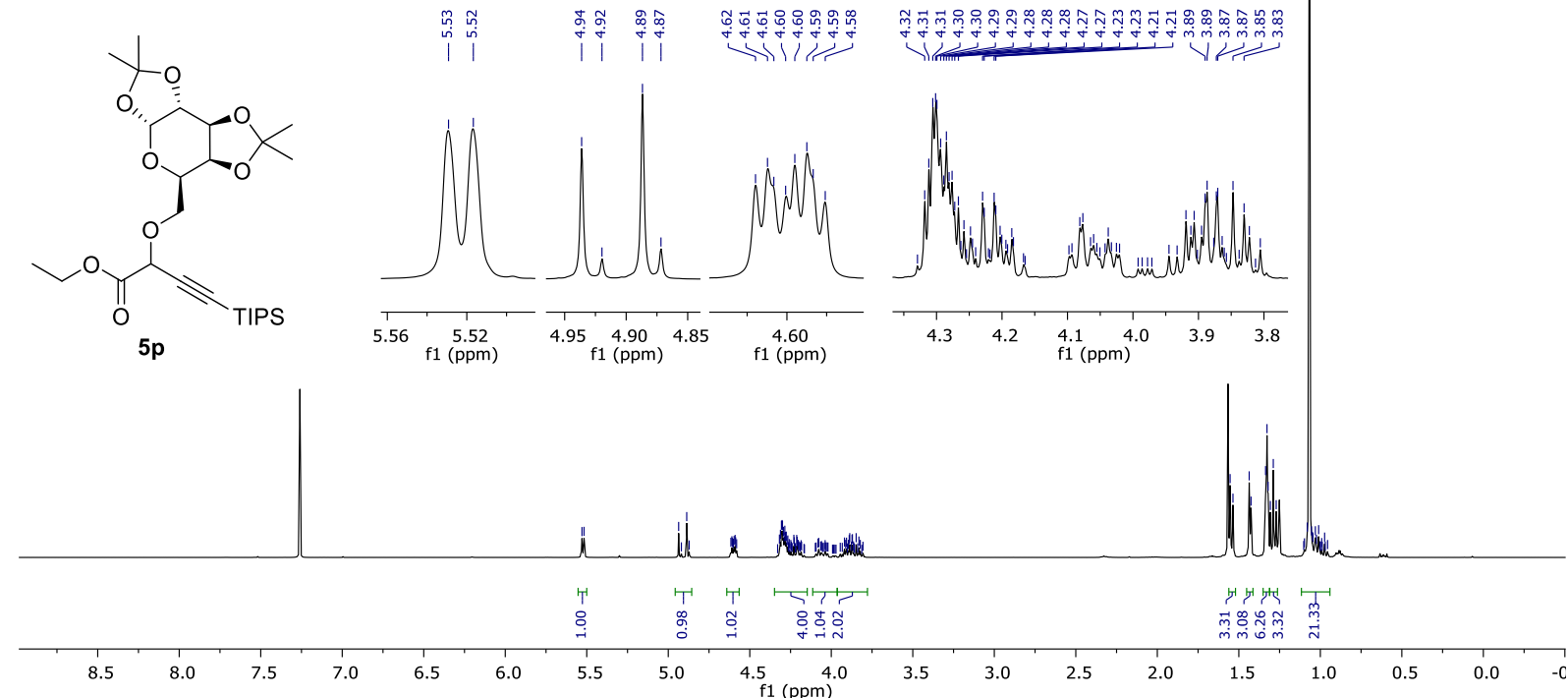

${ }^{13} \mathrm{C}-\mathrm{NMR}\left(101 \mathrm{MHz}, \mathrm{CDCl}_{3}\right)$ of compound $\mathbf{5 p}$

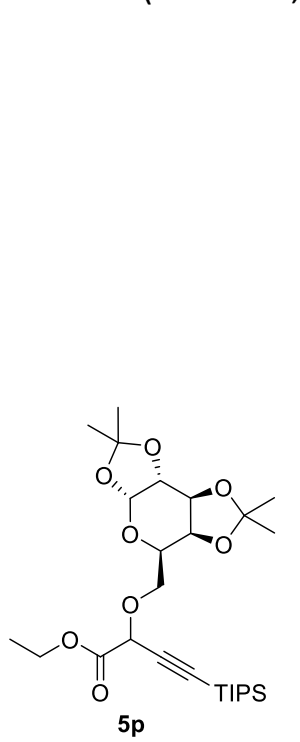

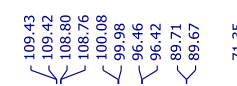

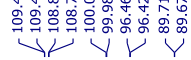

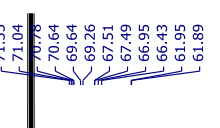

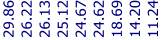

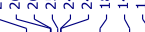

$\begin{array}{lllllllllll}210 & 200 & 190 & 180 & 170 & 160 & 150 & 140 & 130 & 120 & 110 \underset{\mathrm{f} 1(\mathrm{ppm})}{100}\end{array}$
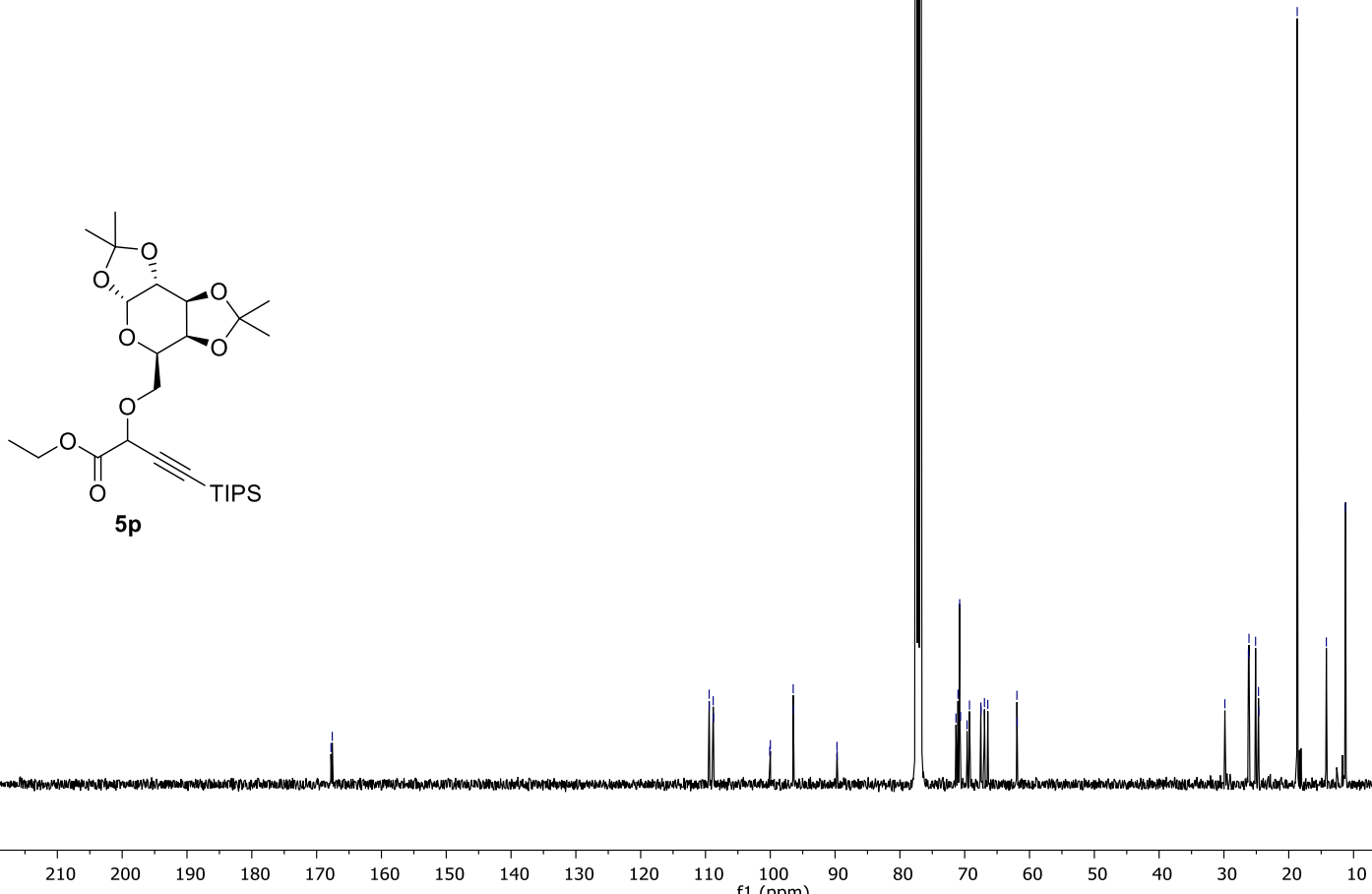

70

50

30

10 
${ }^{1} \mathbf{H}-\mathrm{NMR}\left(400 \mathrm{MHz}, \mathrm{CDCl}_{3}\right)$ of compound $\mathbf{5 q}$

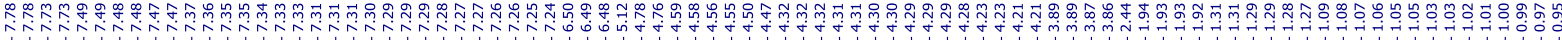

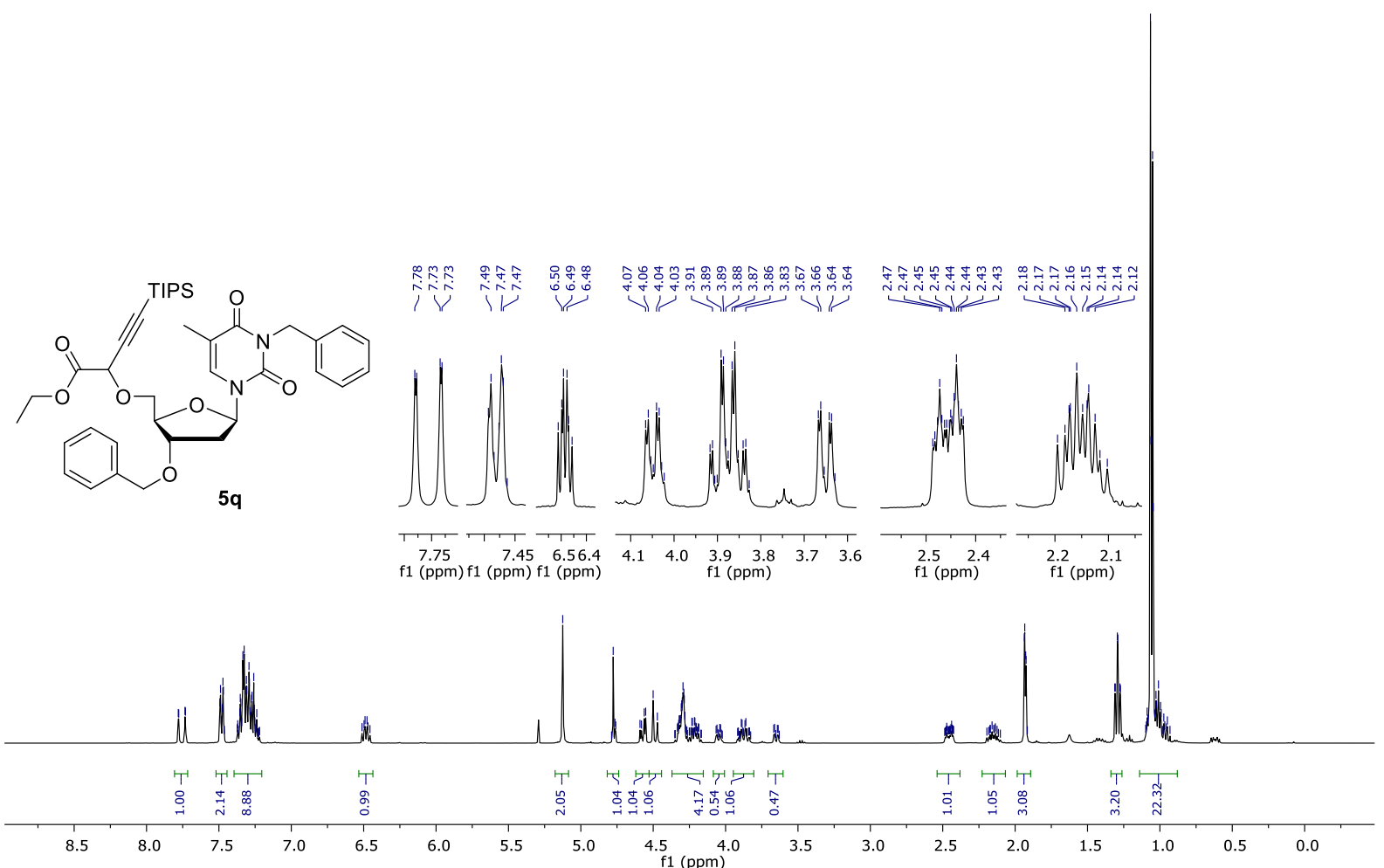

${ }^{13} \mathrm{C}-\mathrm{NMR}\left(101 \mathrm{MHz}, \mathrm{CDCl}_{3}\right)$ of compound $\mathbf{5 q}$

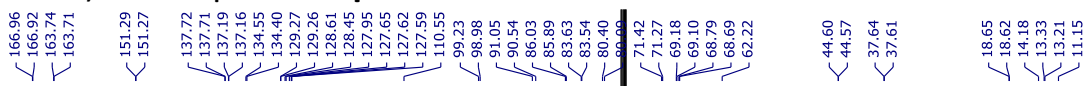

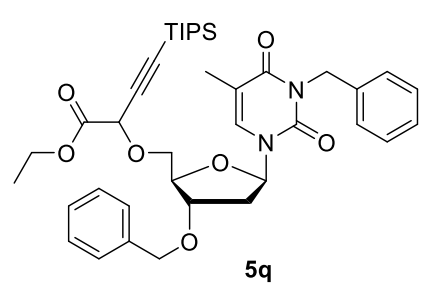


${ }^{1}$ H-NMR $\left(400 \mathrm{MHz}, \mathrm{CDCl}_{3}\right)$ of compound $\mathbf{5 r}$

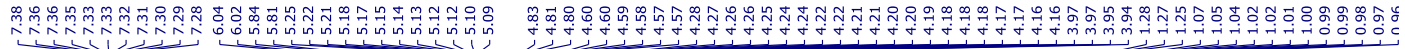
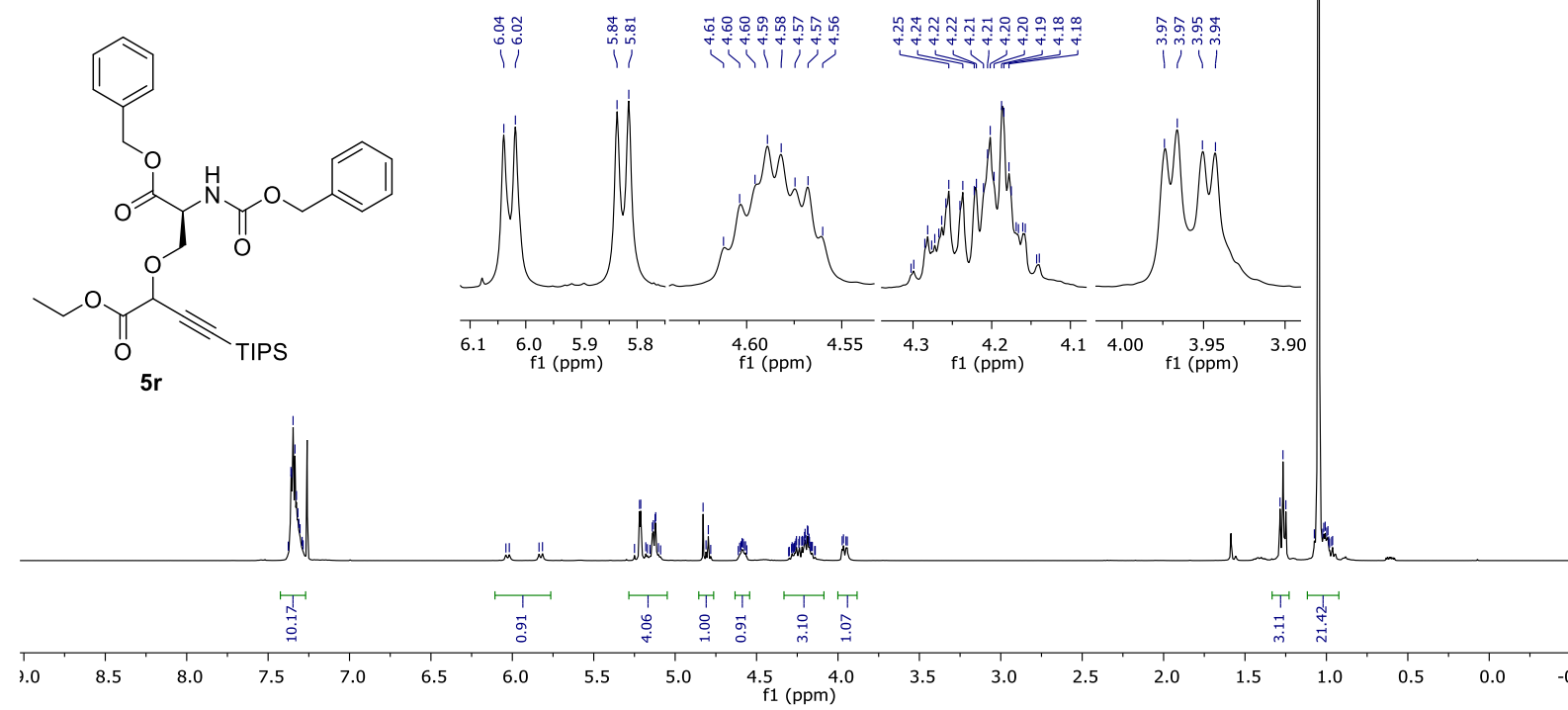

${ }^{13} \mathrm{C}$-NMR $\left(101 \mathrm{MHz}, \mathrm{CDCl}_{3}\right.$ ) of compound $5 \mathrm{r}$

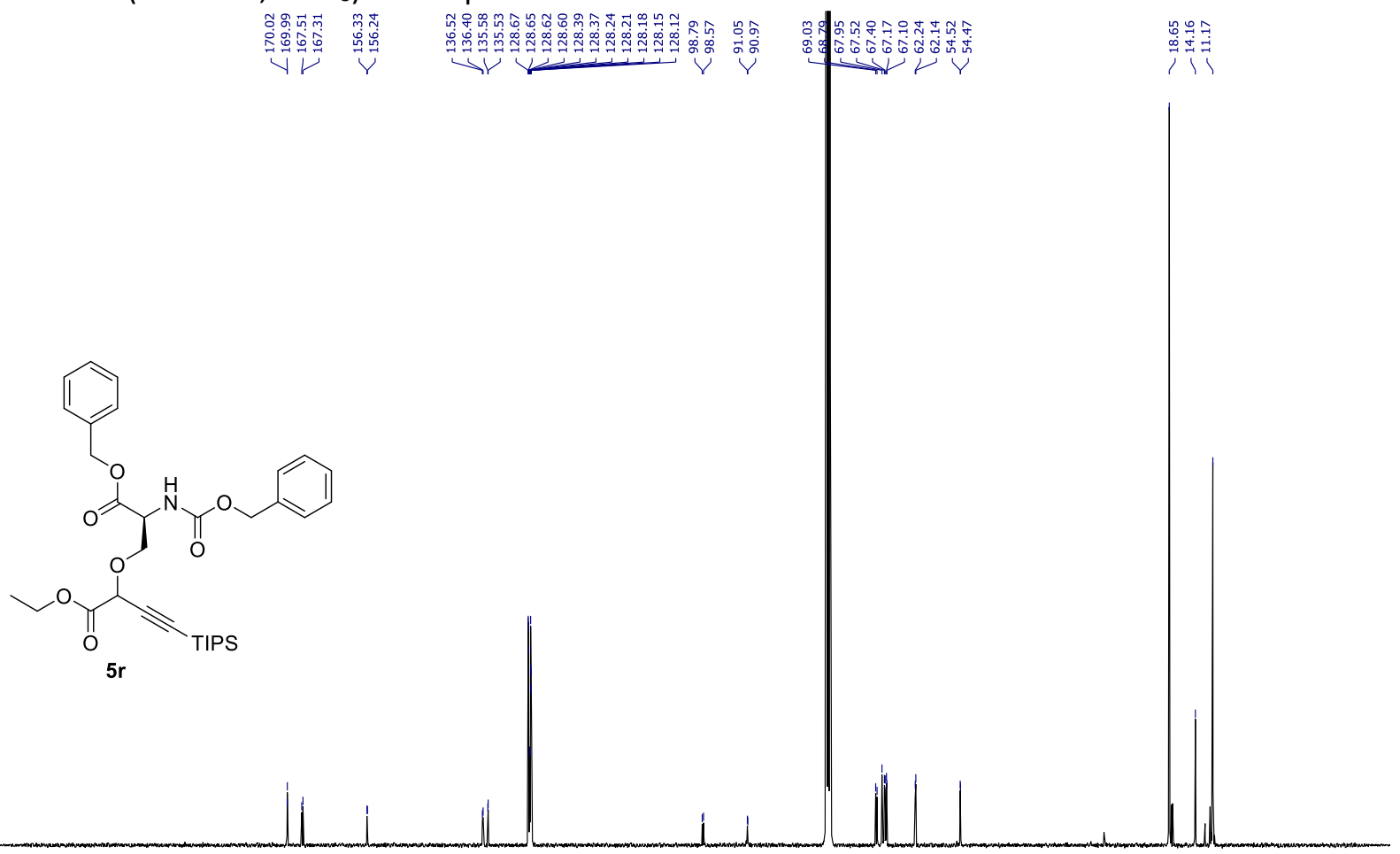

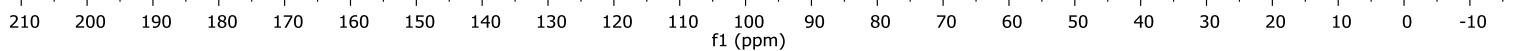


${ }^{1}$ H-NMR (400 MHz, $\mathrm{CDCl}_{3}$ ) of compound 5 s

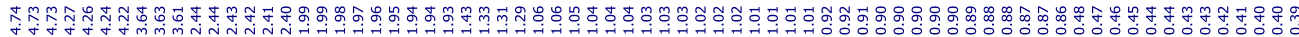

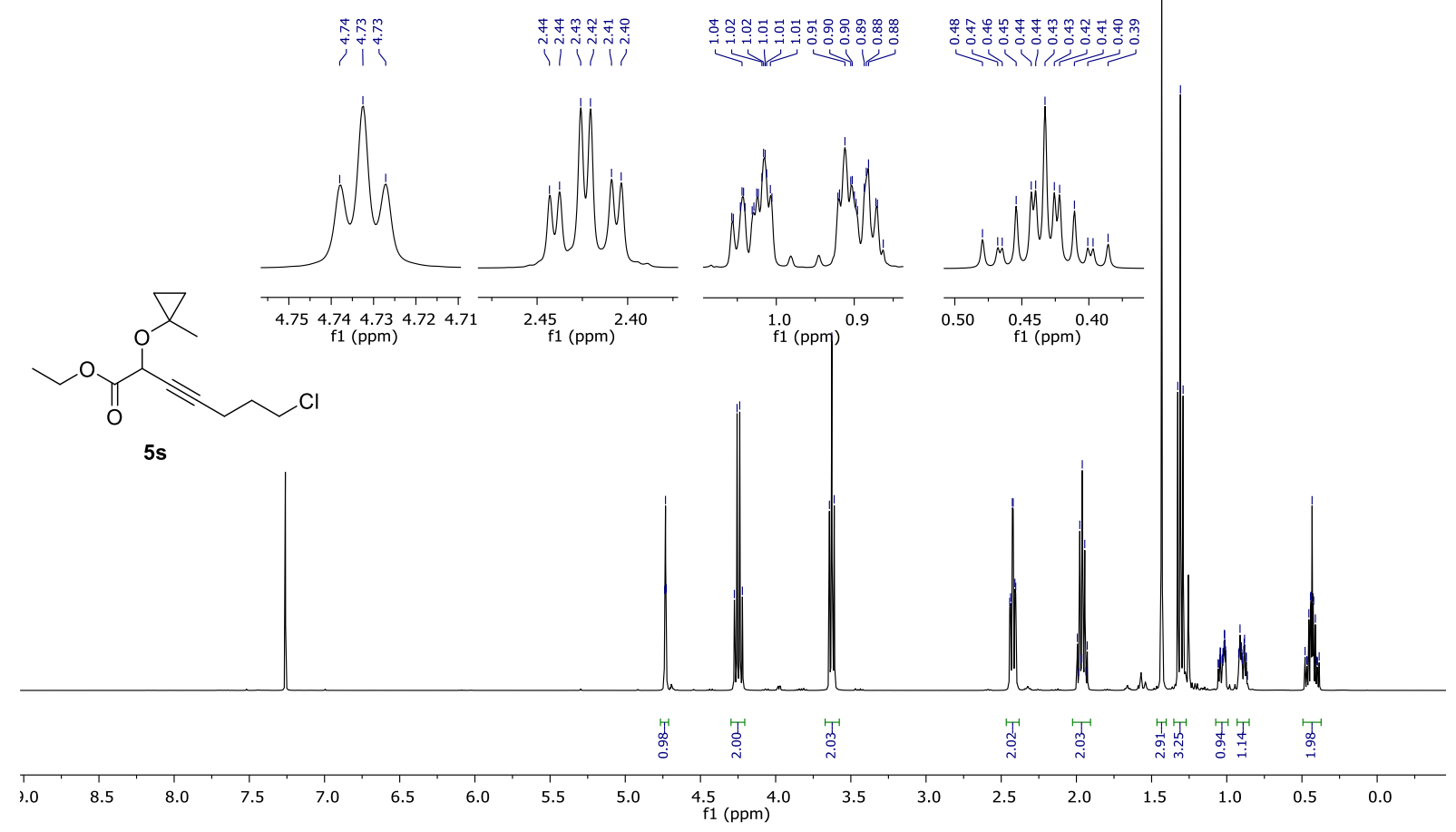

${ }^{13} \mathrm{C}-\mathrm{NMR}\left(101 \mathrm{MHz}, \mathrm{CDCl}_{3}\right)$ of compound $\mathbf{5 s}$

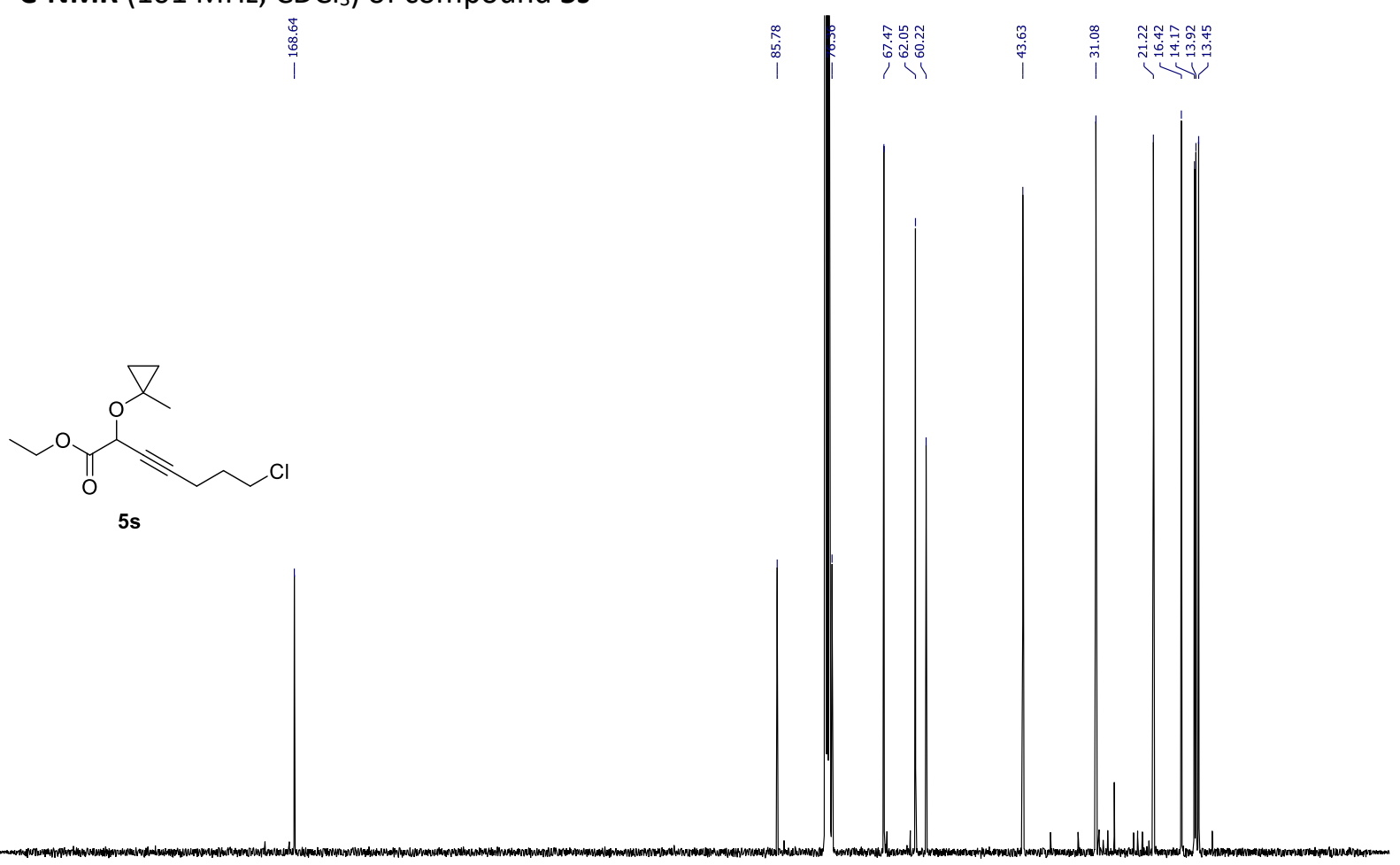

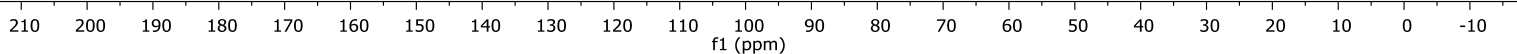


${ }^{1} \mathbf{H}-\mathrm{NMR}\left(400 \mathrm{MHz}, \mathrm{CDCl}_{3}\right)$ of compound $\mathbf{5 t}$

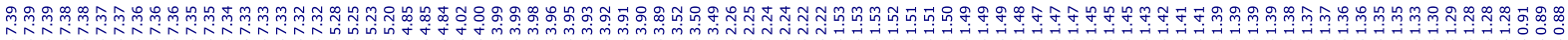

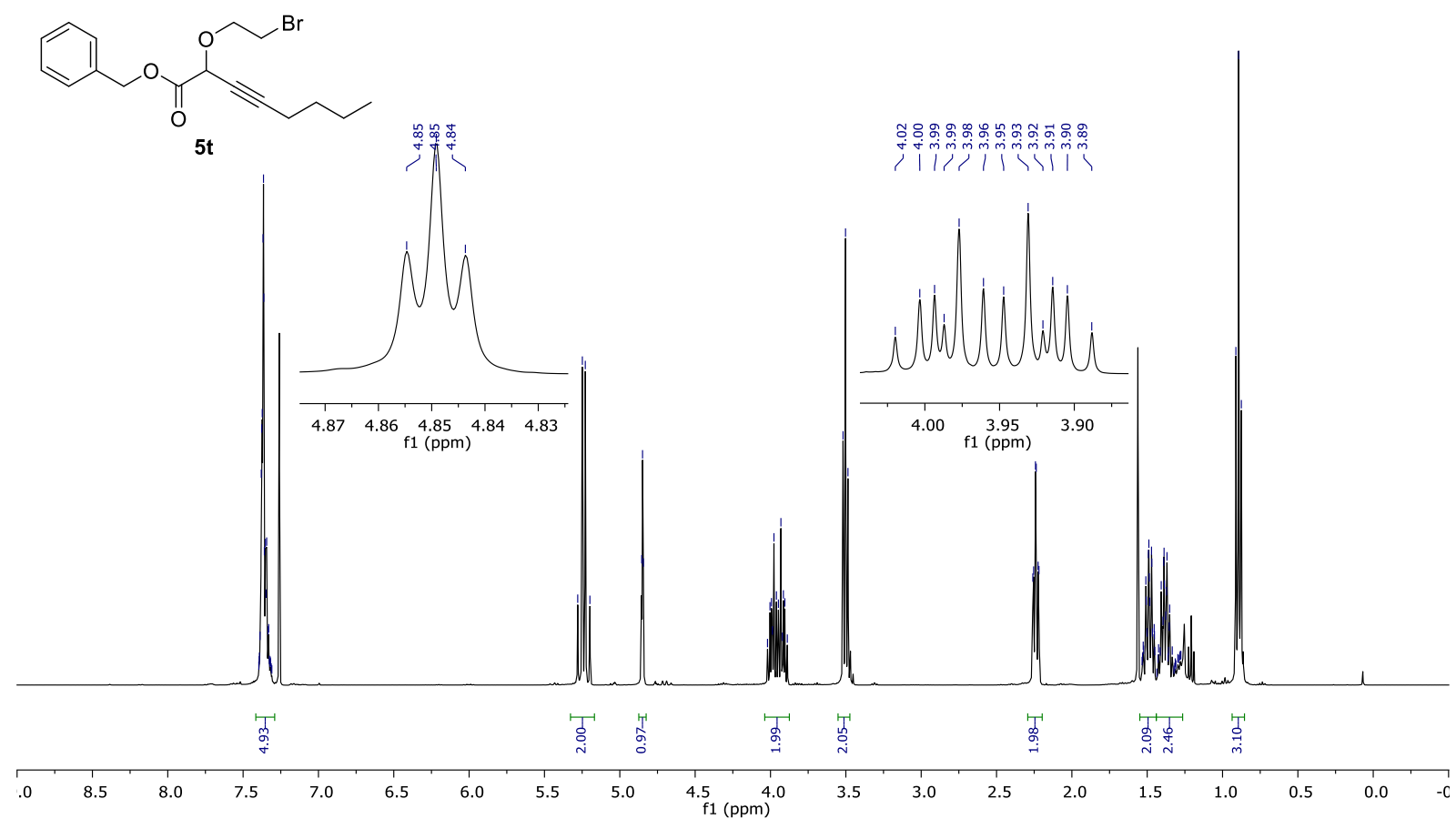

${ }^{13} \mathrm{C}-\mathrm{NMR}\left(101 \mathrm{MHz}, \mathrm{CDCl}_{3}\right)$ of compound $\mathbf{5 t}$

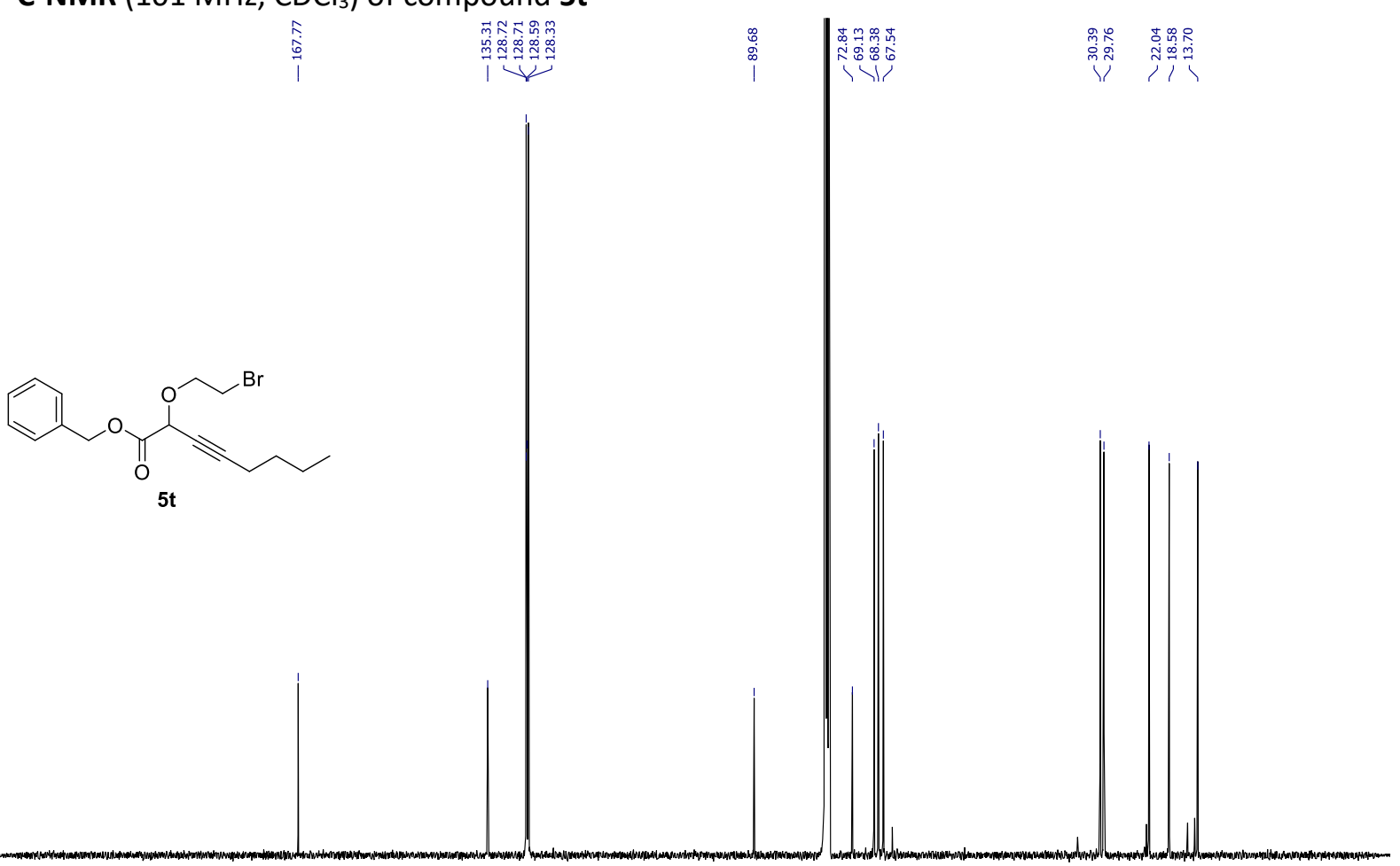

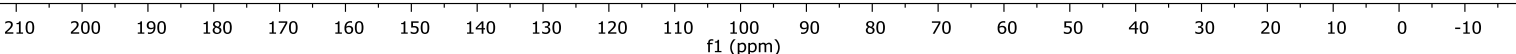


${ }^{1} \mathbf{H}-\mathrm{NMR}\left(400 \mathrm{MHz}, \mathrm{CDCl}_{3}\right)$ of compound $\mathbf{5 u}$

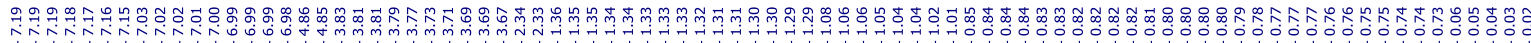<smiles>CCCCOC(C#CC1CC1)C(=O)Oc1ccc(C)cc1</smiles>
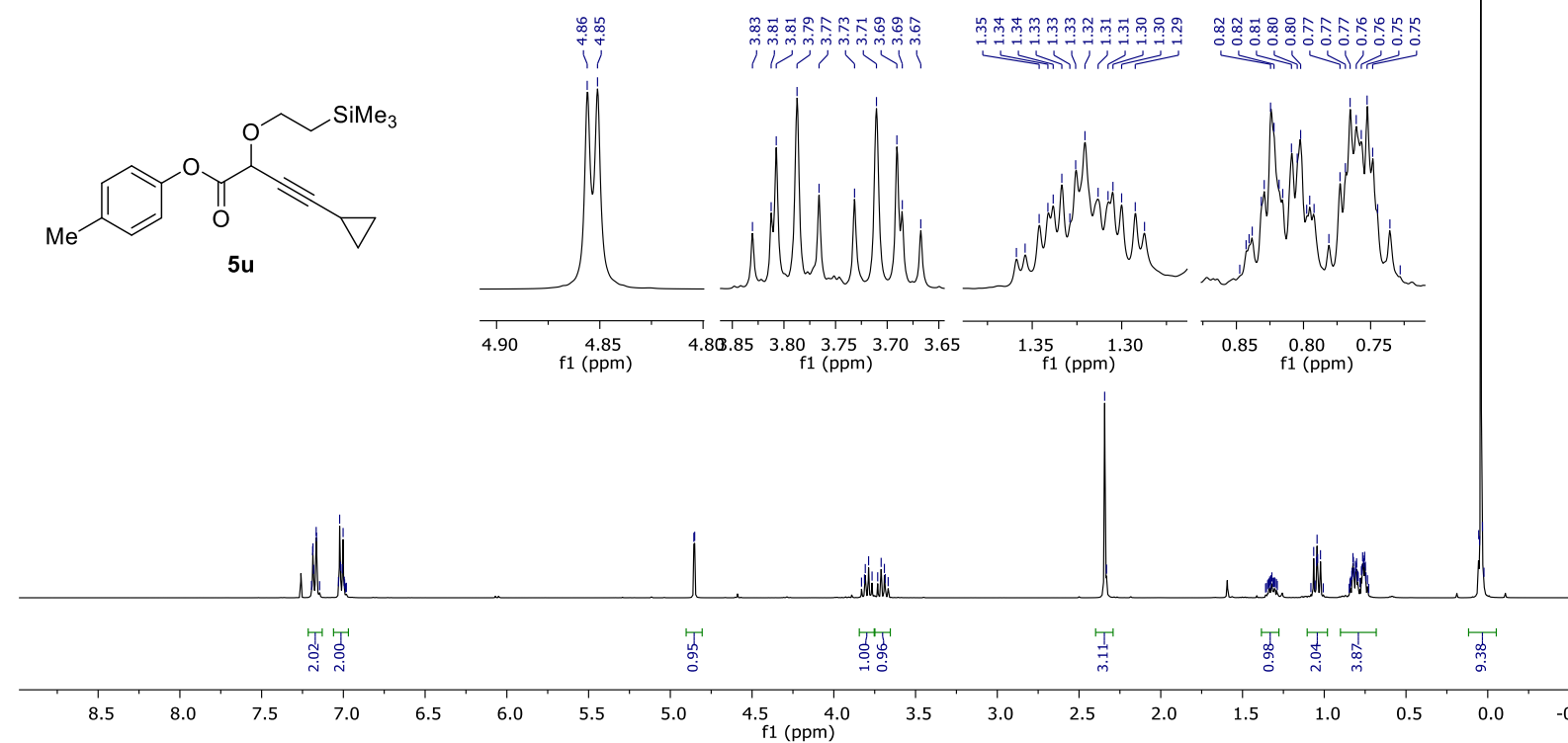

${ }^{13}$ C-NMR (101 MHz, $\mathrm{CDCl}_{3}$ ) of compound $\mathbf{5 u}$

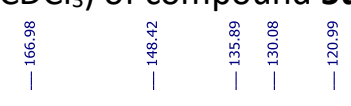

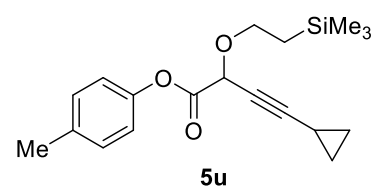

5u

$\begin{array}{lllllllllll}210 & 200 & 190 & 180 & 170 & 160 & 150 & 140 & 130 & 120 & 110 \\ \mathrm{f} 1(\mathrm{ppm})\end{array}$ 
${ }^{1} \mathbf{H}-\mathrm{NMR}\left(400 \mathrm{MHz}, \mathrm{CDCl}_{3}\right.$ ) of compound $\mathbf{5 v}$ 节
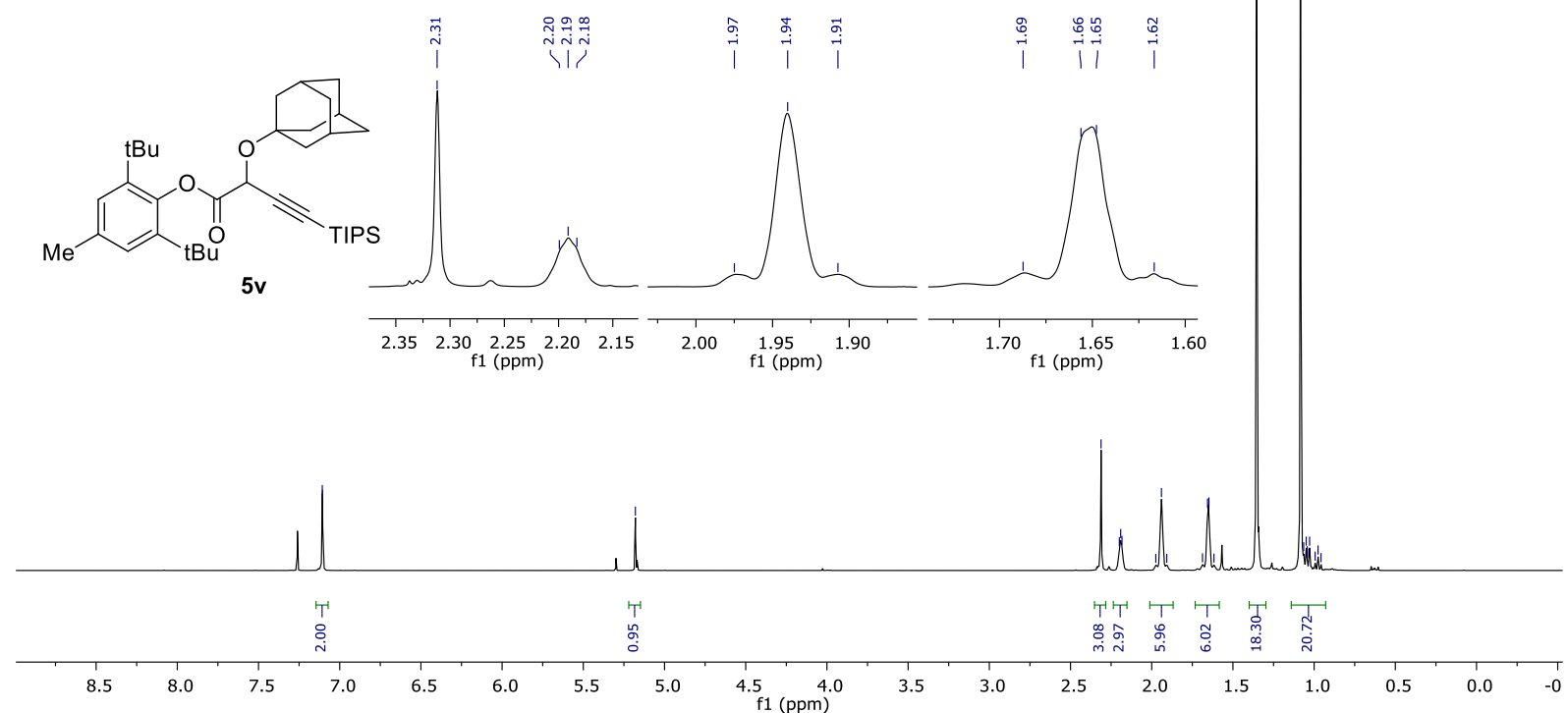

${ }^{13}$ C-NMR (101 MHz, $\mathrm{CDCl}_{3}$ ) of compound 5v

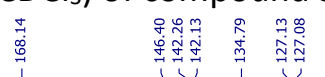<smiles>Cc1cc(Br)c(OC(=O)C(C#CC(=O)c2ccccc2)OC2=CC3CCC(C2)C3)c(Br)c1</smiles>

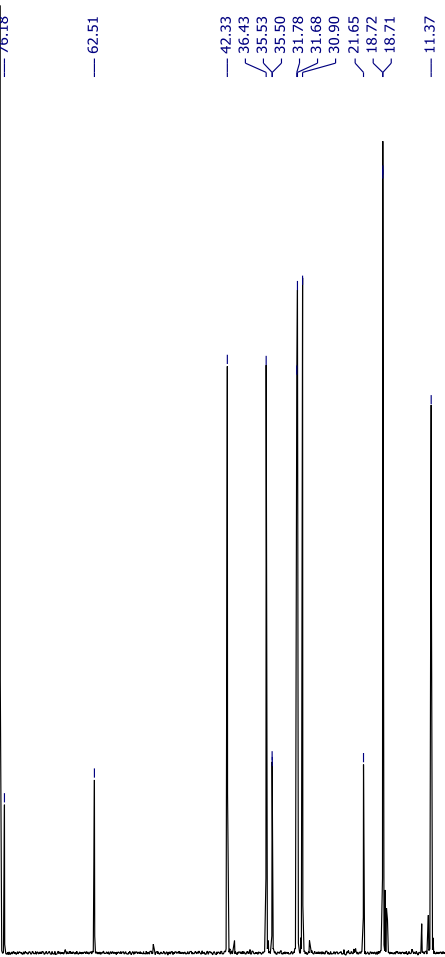

$\begin{array}{lllllllllll}210 & 200 & 190 & 180 & 170 & 160 & 150 & 140 & 130 & 120 & 110 \\ \begin{array}{l}100 \\ (\mathrm{ppm})\end{array}\end{array}$ 

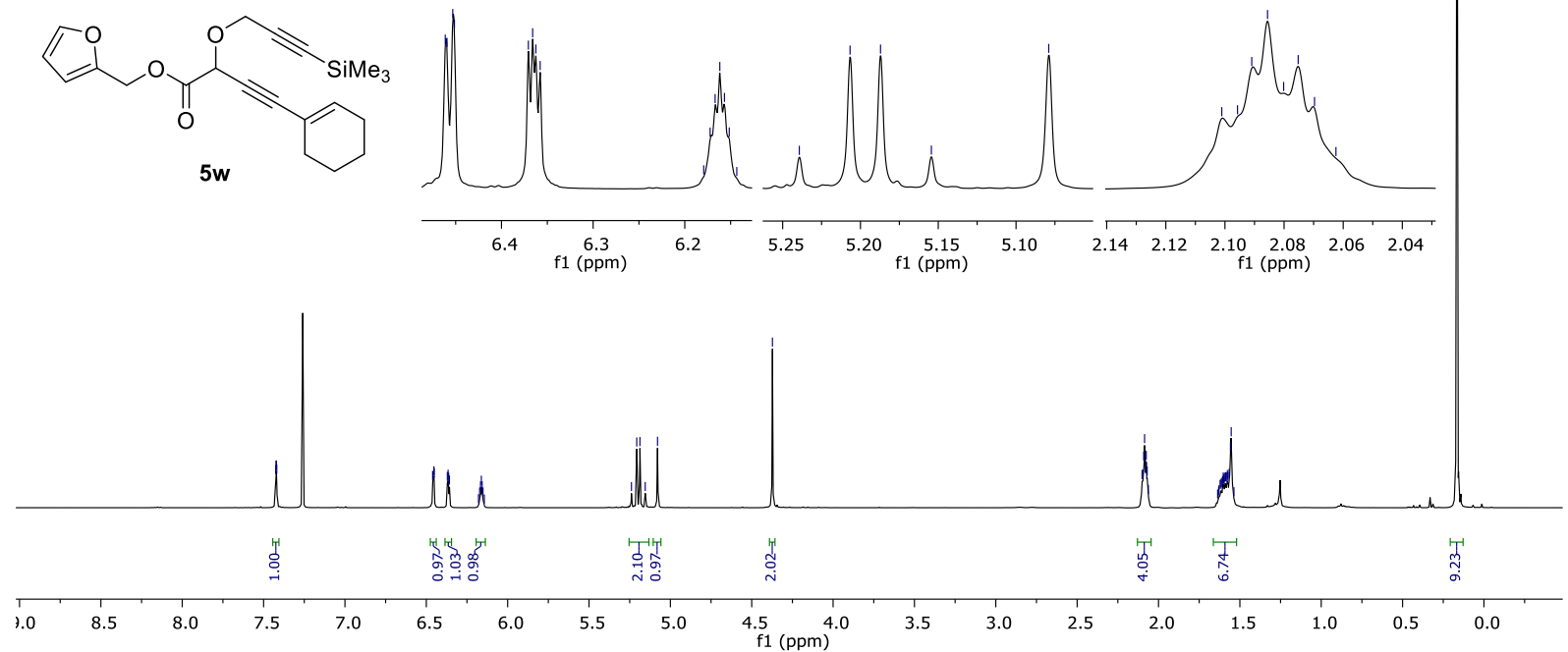

${ }^{13} \mathrm{C}-\mathrm{NMR}\left(101 \mathrm{MHz}, \mathrm{CDCl}_{3}\right.$ ) of compound $\mathbf{5 w}$

$$
\text { ind }
$$

$5 w$
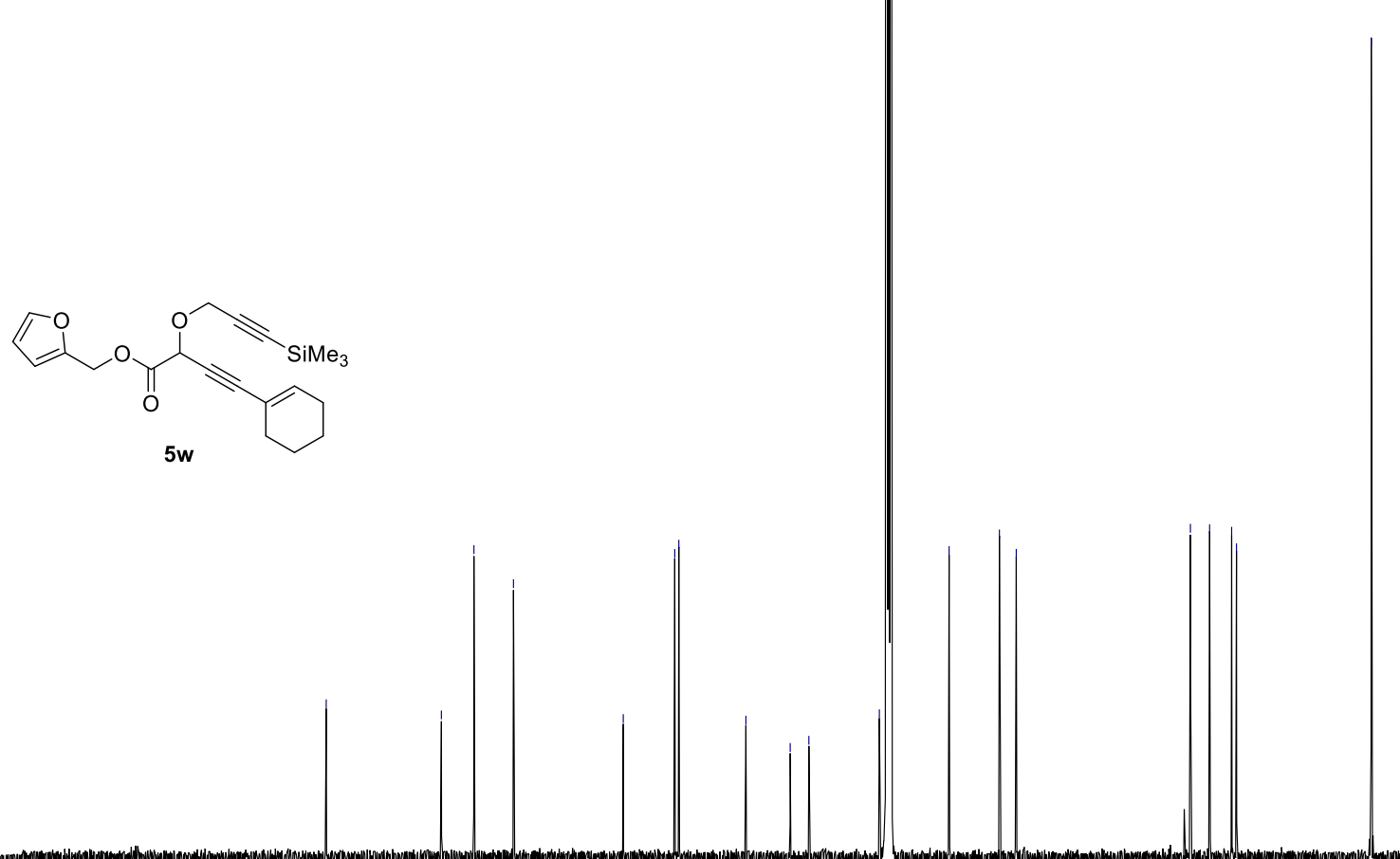

$\begin{array}{lllllllllll}210 & 200 & 190 & 180 & 170 & 160 & 150 & 140 & 130 & 120 & 110 \\ \mathrm{f} 1(\mathrm{ppm})\end{array}$ 
${ }^{1} \mathbf{H}-\mathrm{NMR}\left(400 \mathrm{MHz}, \mathrm{CDCl}_{3}\right)$ of compound $\mathbf{5 x}$

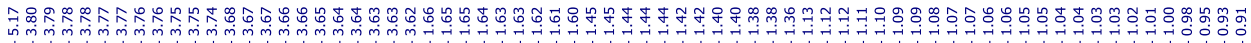

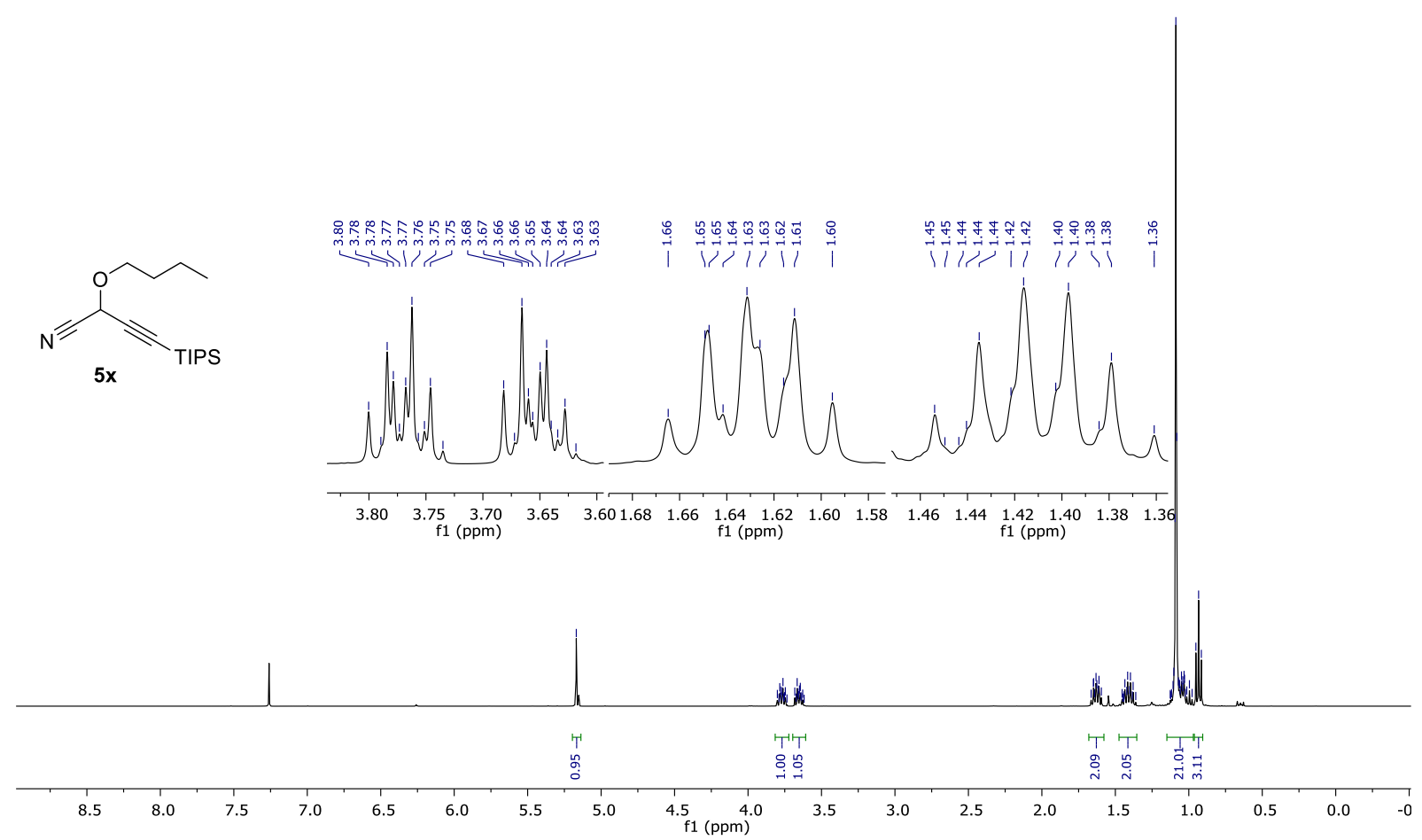

${ }^{13} \mathrm{C}-\mathrm{NMR}\left(101 \mathrm{MHz}, \mathrm{CDCl}_{3}\right.$ ) of compound $\mathbf{5 x}$

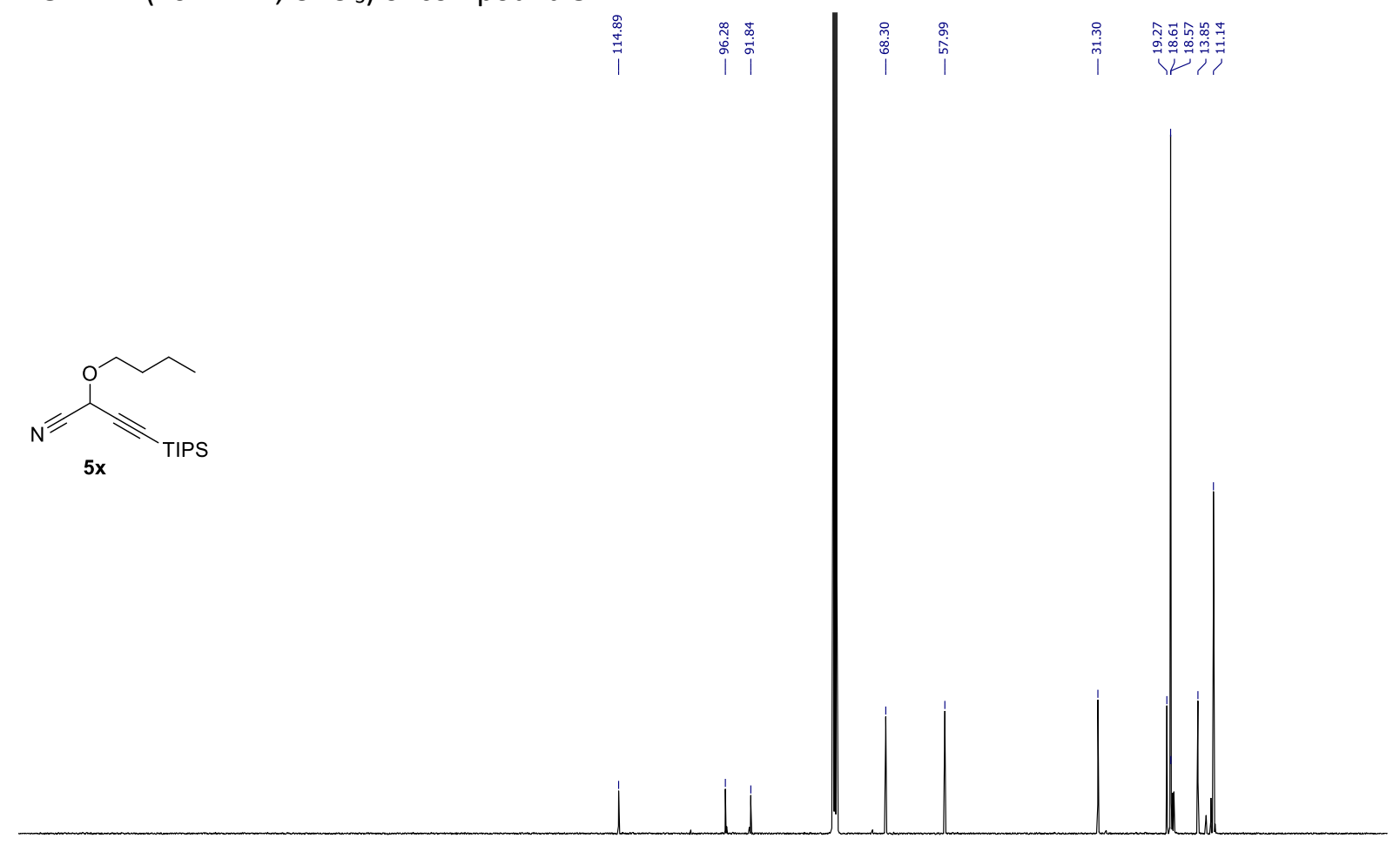

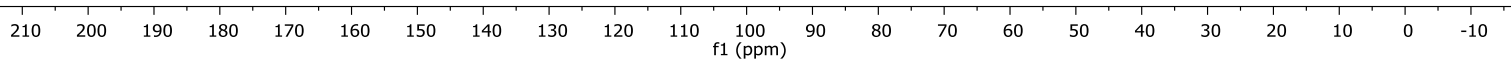


${ }^{1} \mathbf{H}-\mathrm{NMR}\left(400 \mathrm{MHz}, \mathrm{CDCl}_{3}\right)$ of compound $\mathbf{5 y}$

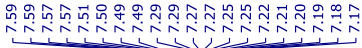

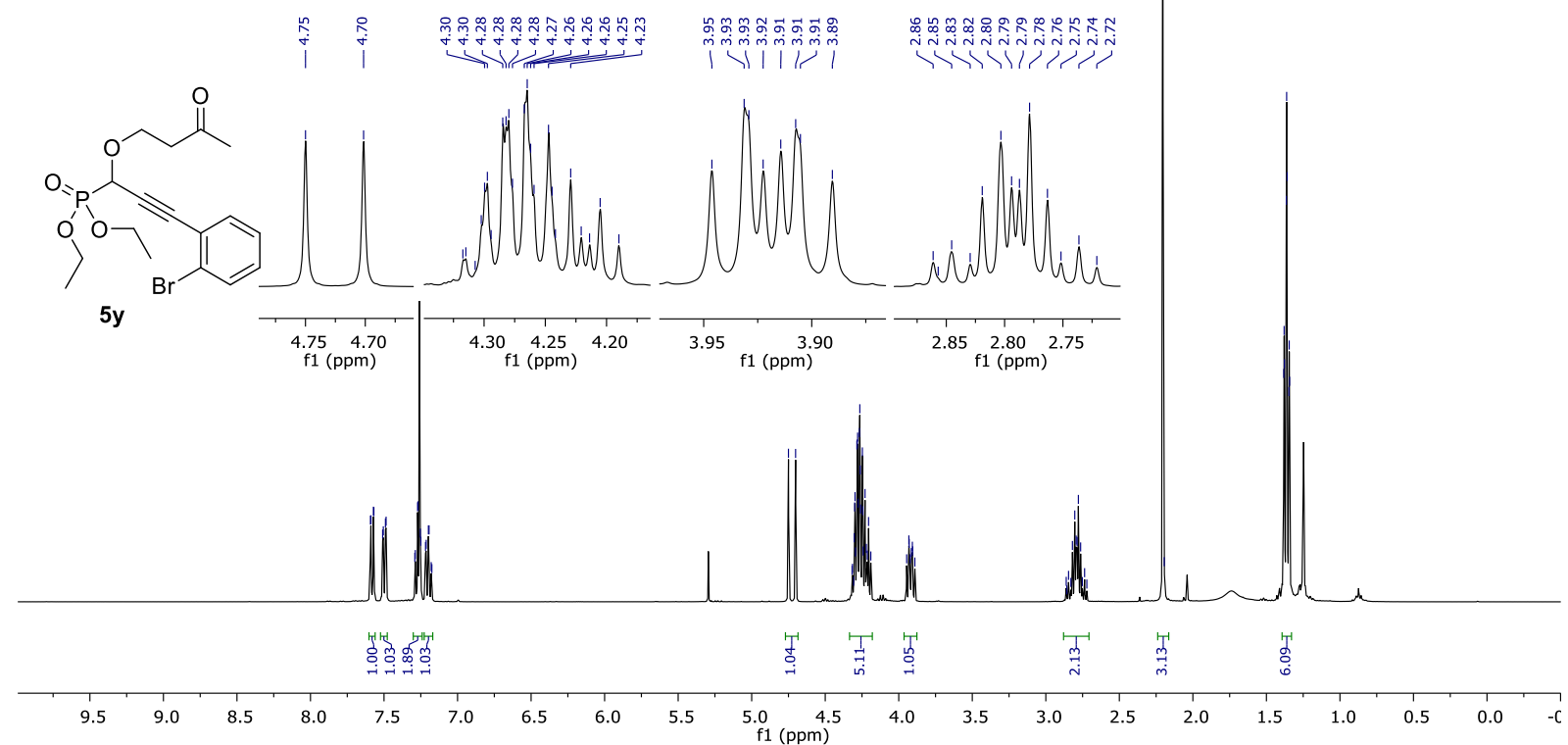

${ }^{13} \mathrm{C}$-NMR (101 MHz, $\mathrm{CDCl}_{3}$ ) of compound 5y
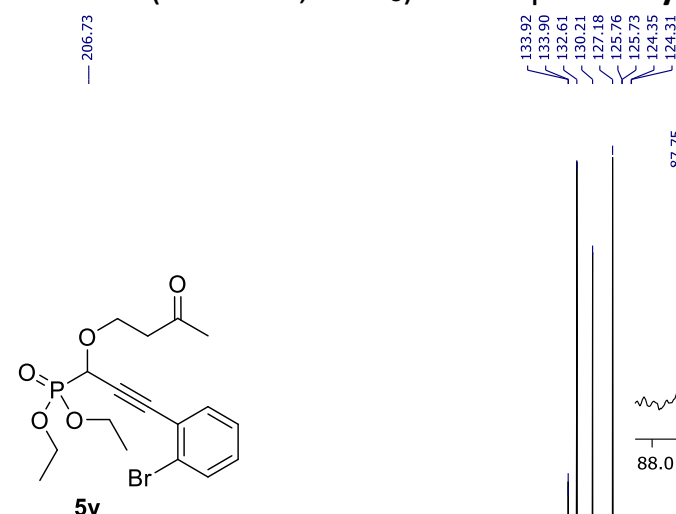

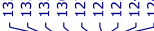

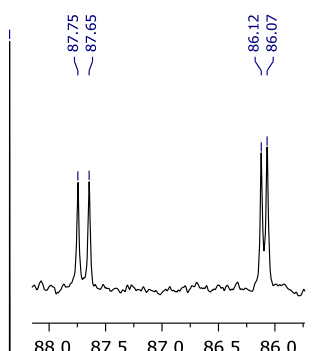

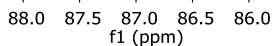

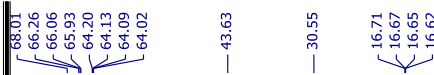

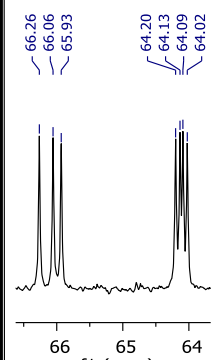

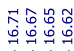

111

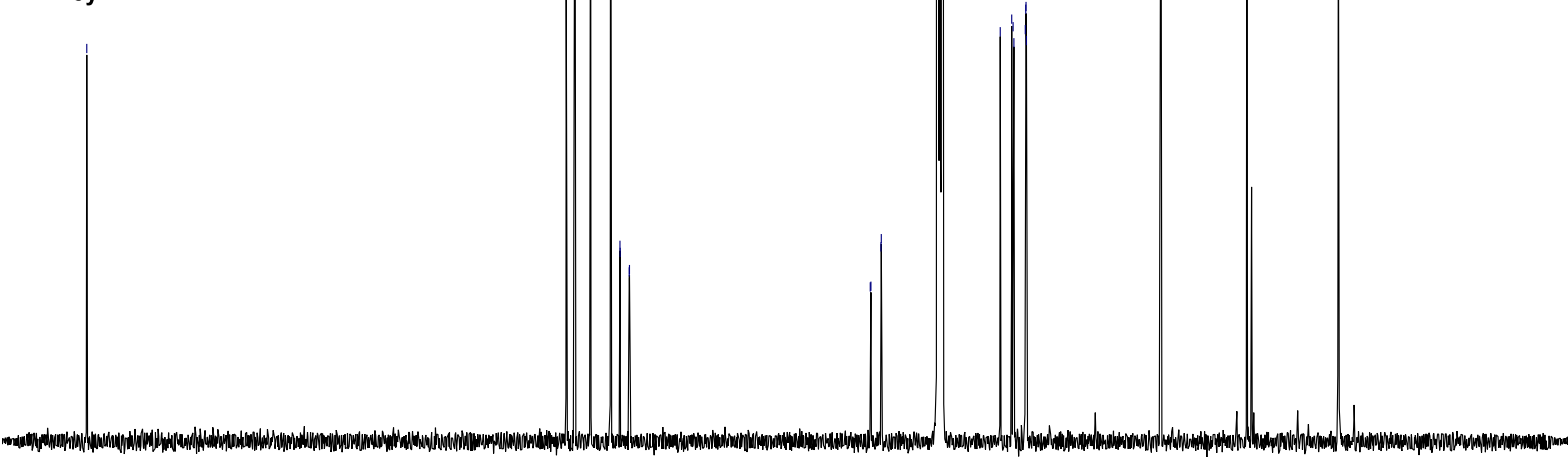

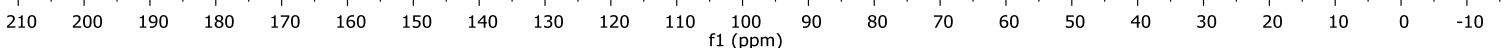


${ }^{31}$ P-NMR (162 MHz, $\mathrm{CDCl}_{3}$ ) of compound 5y

$\stackrel{8}{1}$

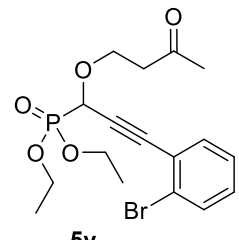

$5 y$

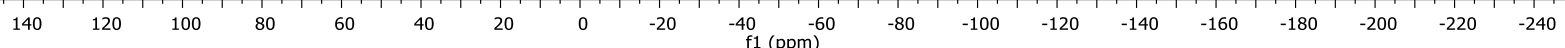




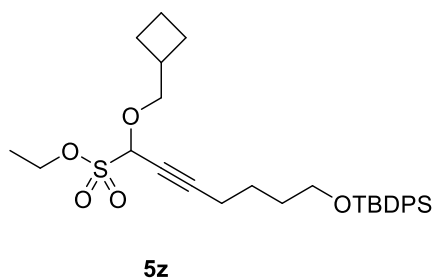

$5 z$

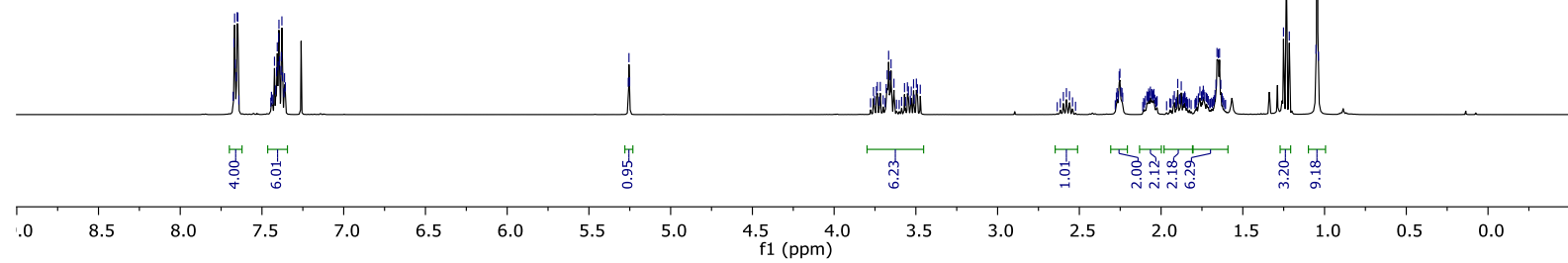

${ }^{13} \mathrm{C}-\mathrm{NMR}\left(101 \mathrm{MHz}, \mathrm{CDCl}_{3}\right)$ of compound $\mathbf{5 z}$

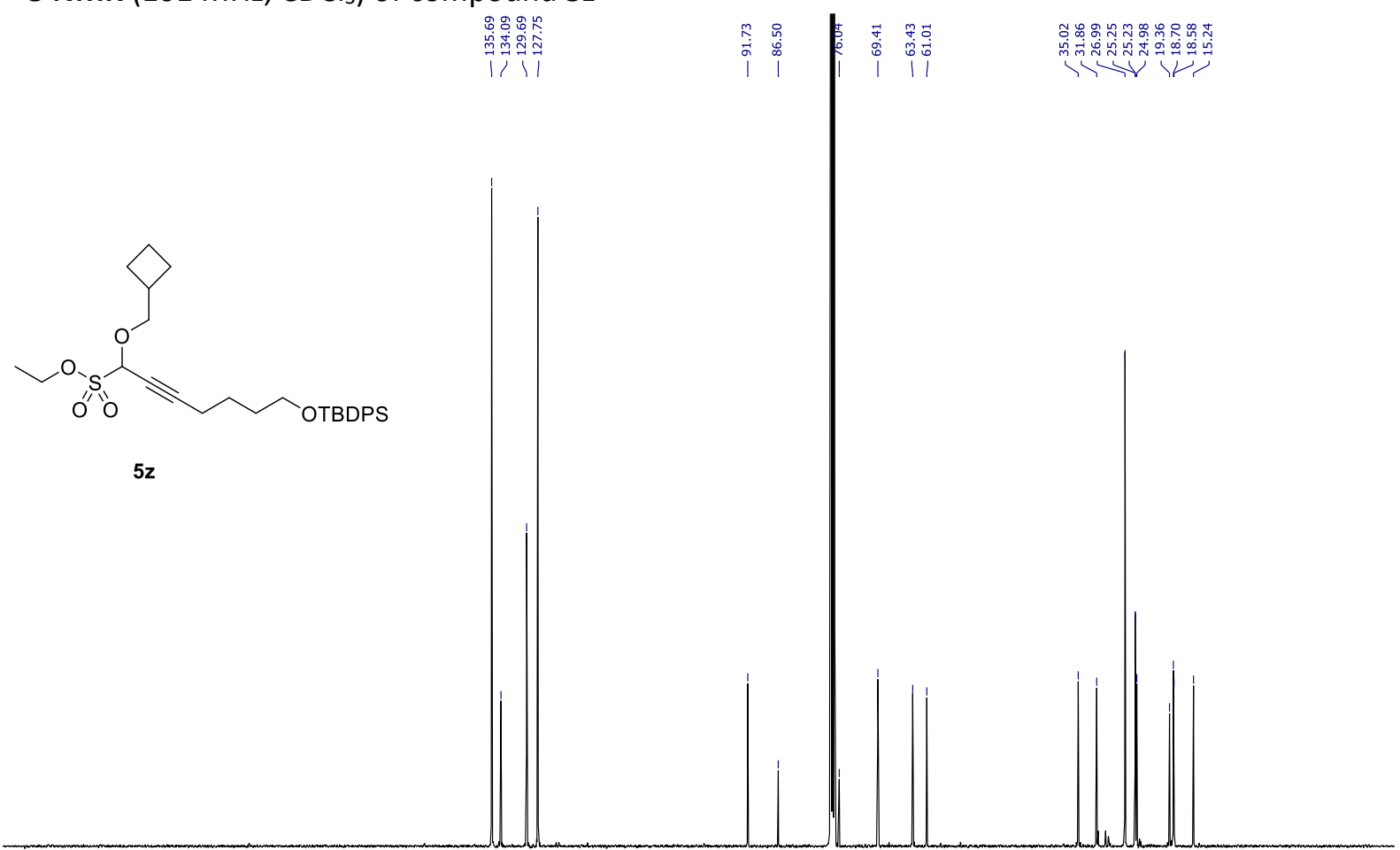

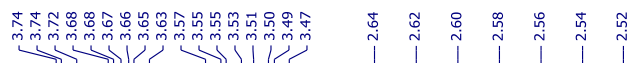

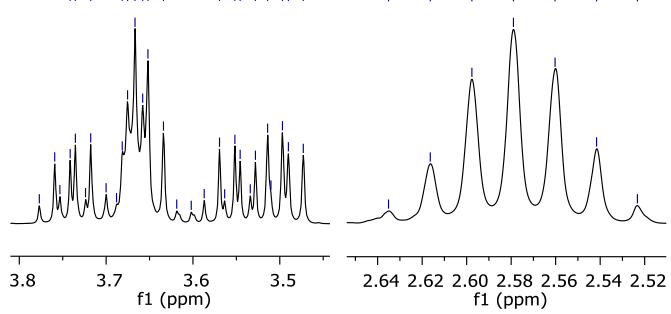


${ }^{1} \mathrm{H}-\mathrm{NMR}\left(400 \mathrm{MHz}, \mathrm{CDCl}_{3}\right.$ ) of compound 5 aa

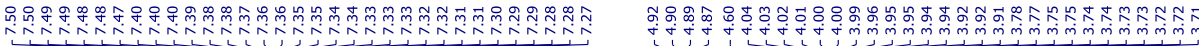

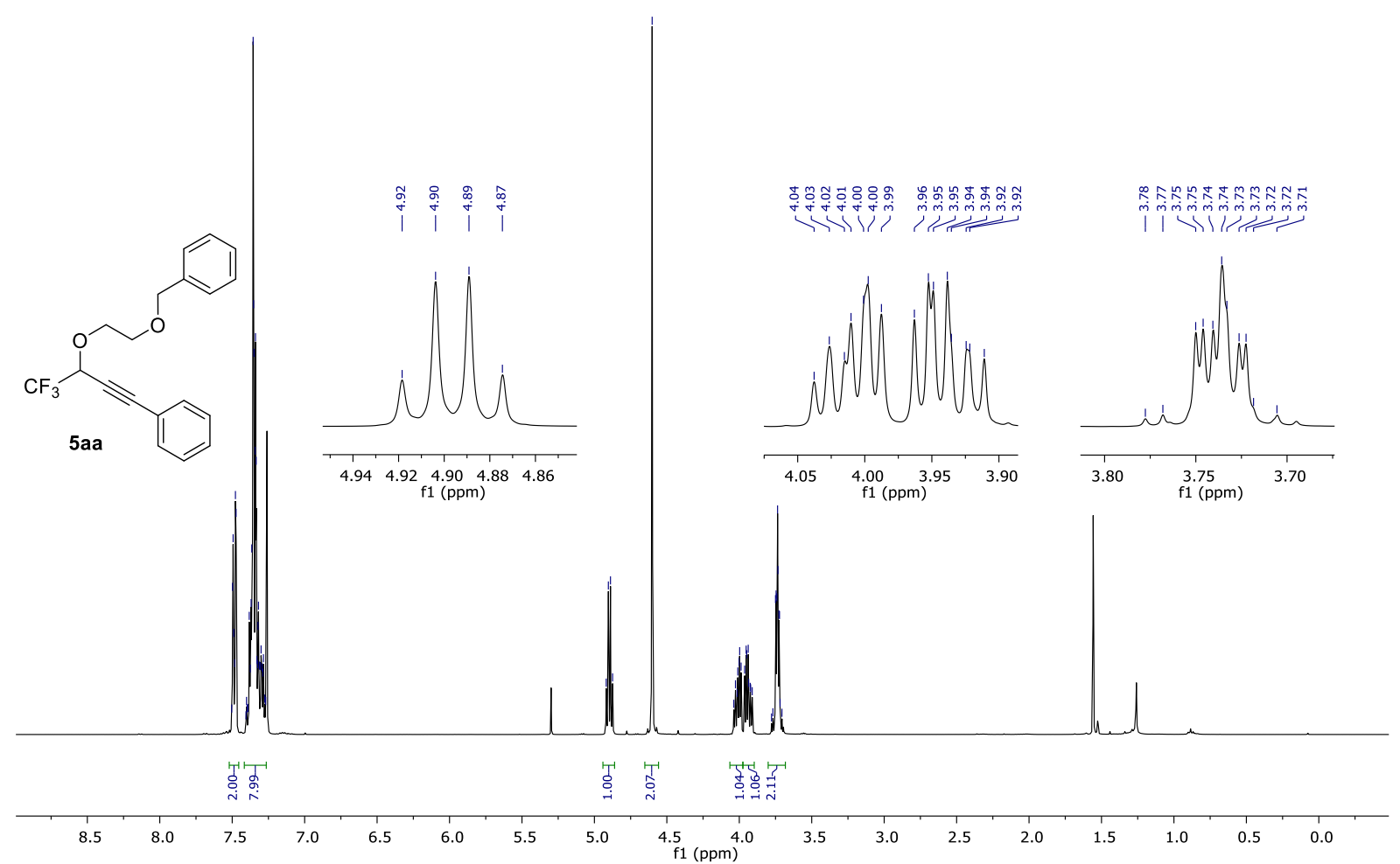

${ }^{13} \mathrm{C}$-NMR (101 MHz, $\mathrm{CDCl}_{3}$ ) of compound 5 aa

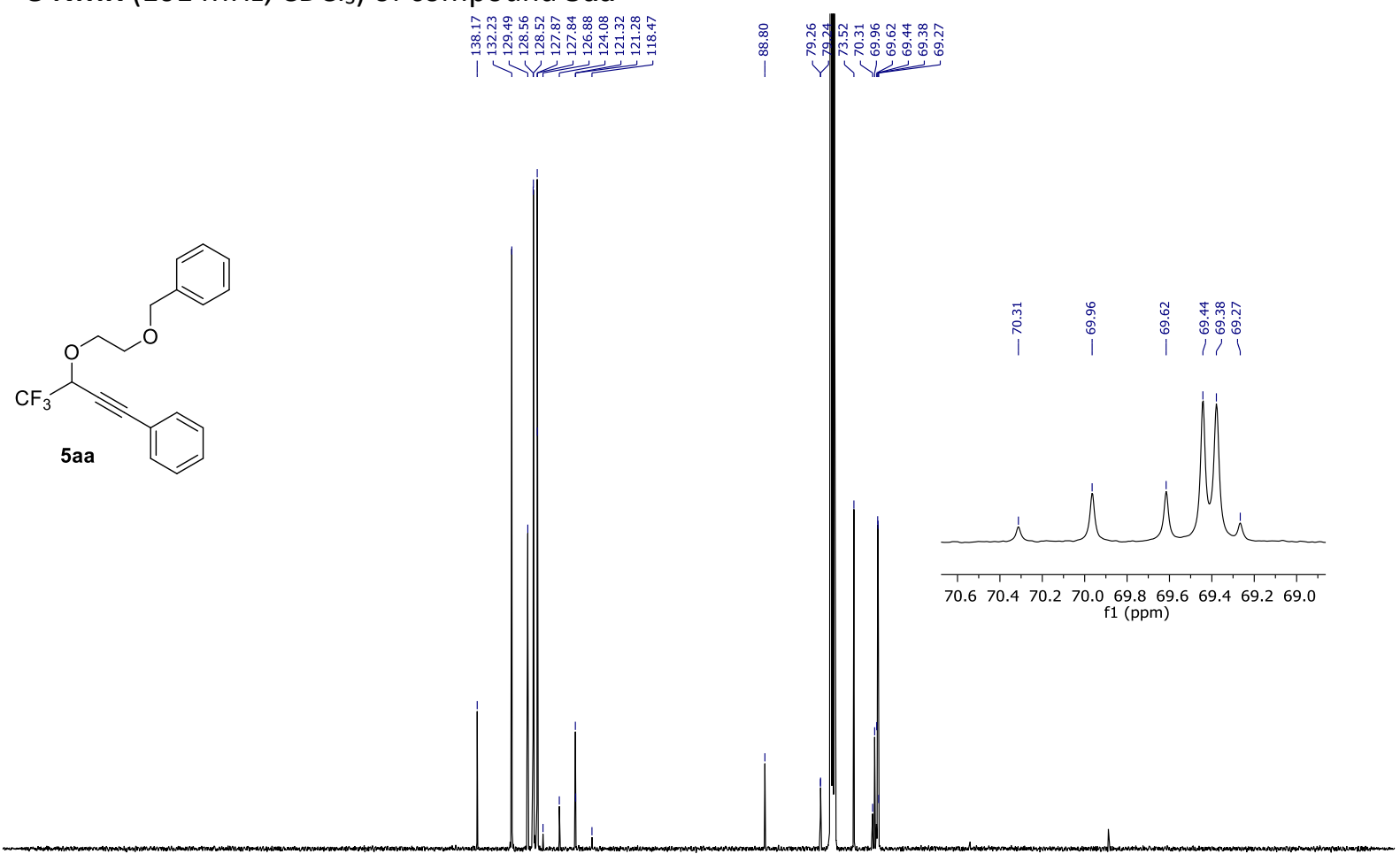

$\begin{array}{lllllllllllllllllllllll}210 & 200 & 190 & 180 & 170 & 160 & 150 & 140 & 130 & 120 & 110 & \begin{array}{c}100 \\ \mathrm{f} 1(\mathrm{ppm})\end{array} & 90 & 80 & 70 & 60 & 50 & 40 & 30 & 20 & 10 & 0 & -10\end{array}$ 
${ }^{19} \mathrm{~F}-\mathrm{NMR}\left(376 \mathrm{MHz}, \mathrm{CDCl}_{3}\right.$ ) of compound $5 \mathrm{aa}$<smiles>CC(C#Cc1ccccc1)OCCOc1ccccc1</smiles>

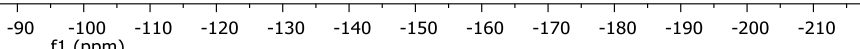


${ }^{1} \mathbf{H}-\mathrm{NMR}\left(400 \mathrm{MHz}, \mathrm{CDCl}_{3}\right.$ ) of compound $\mathbf{5 a b}$

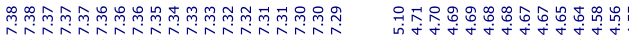
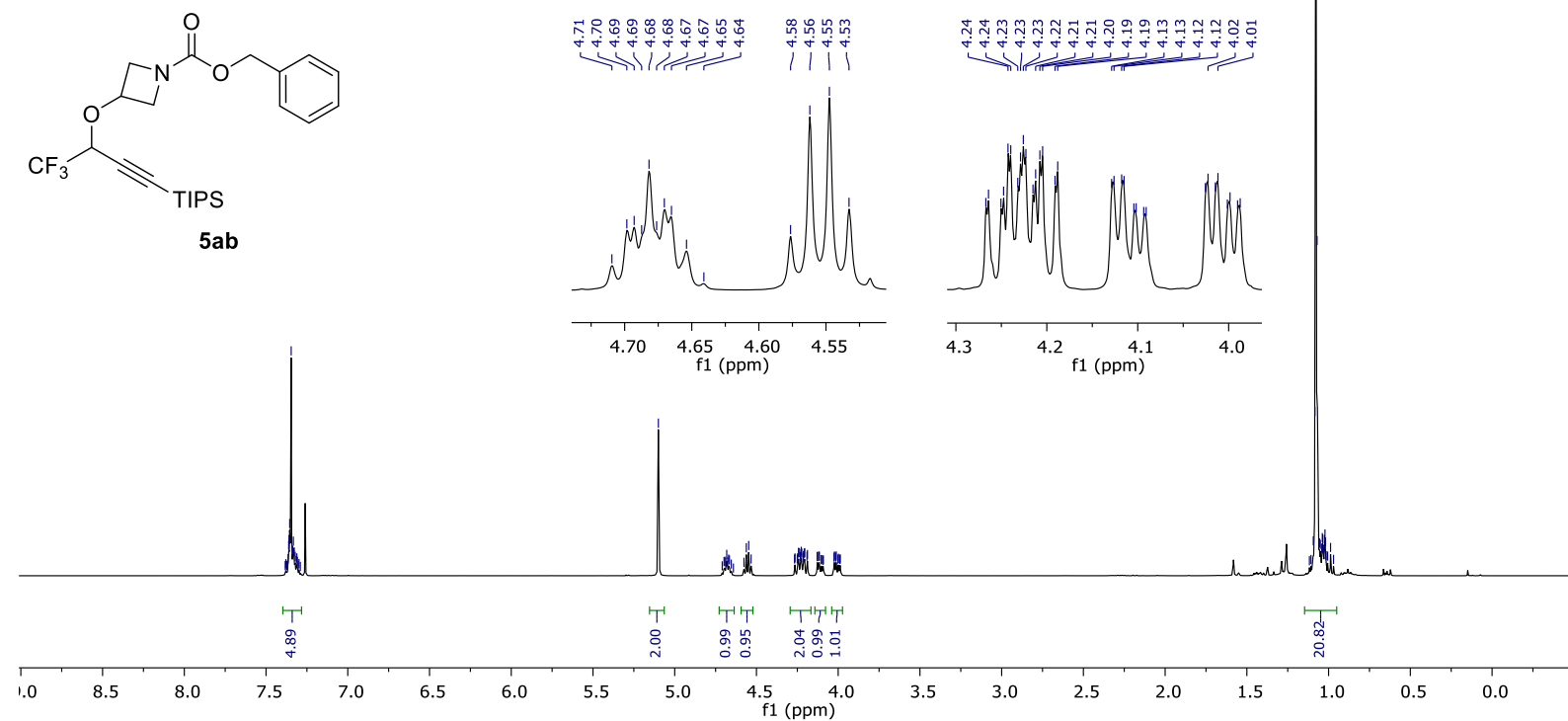

${ }^{13} \mathrm{C}$-NMR (101 MHz, $\mathrm{CDCl}_{3}$ ) of compound $\mathbf{5 a b}$

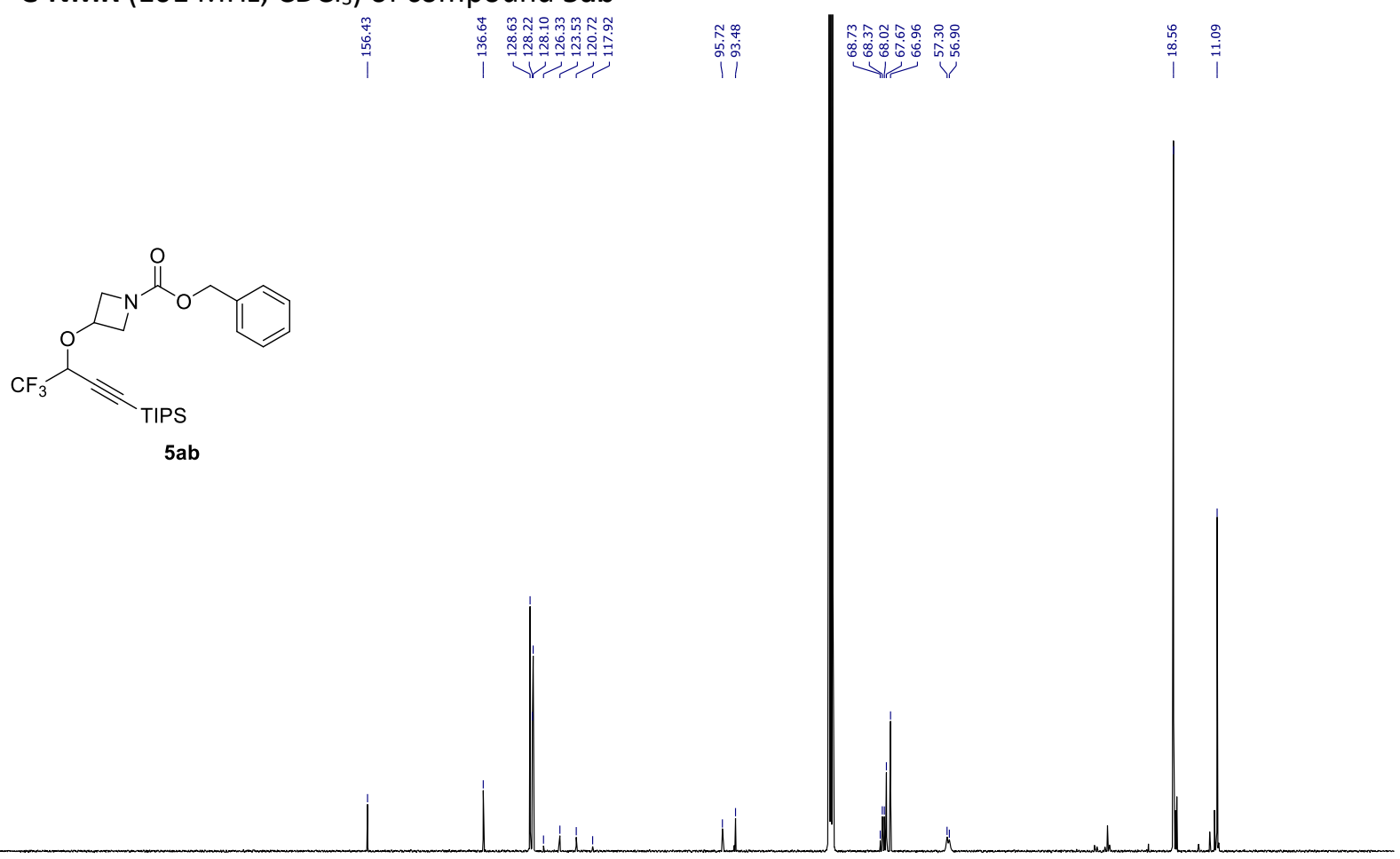

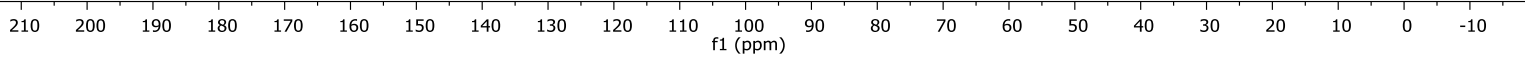


${ }^{19} \mathrm{~F}-\mathrm{NMR}\left(376 \mathrm{MHz}, \mathrm{CDCl}_{3}\right.$ ) of compound $\mathbf{5 a b}$

$\stackrel{\vec{i}}{i}$<smiles>CCCC#CC(OC1CN(C(=O)OCc2ccccc2)C1)C(F)(F)F</smiles>

$5 a b$

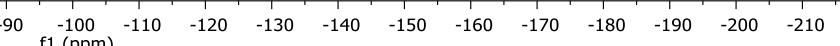


${ }^{1} \mathrm{H}-\mathrm{NMR}\left(400 \mathrm{MHz}, \mathrm{CDCl}_{3}\right.$ ) of compound $\mathbf{5 a c}$

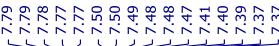

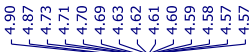

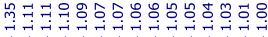
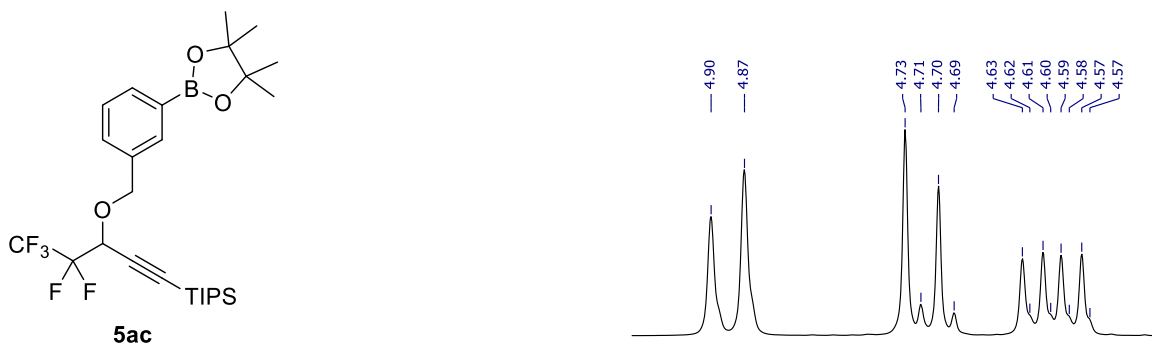

$\begin{array}{lllllllll}4.95 & 4.90 & 4.85 & 4.80 & 4.75 & 4.70 & 4.65 & 4.60 & 4.55\end{array}$

Low

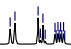

w.

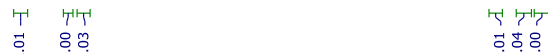

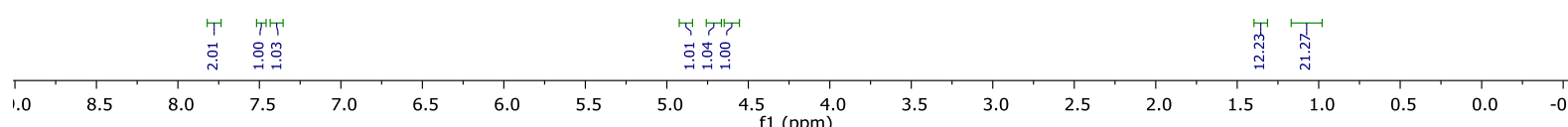

${ }^{13} \mathrm{C}$-NMR (101 MHz, $\mathrm{CDCl}_{3}$ ) of compound $5 \mathrm{ac}$

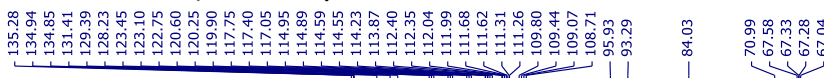

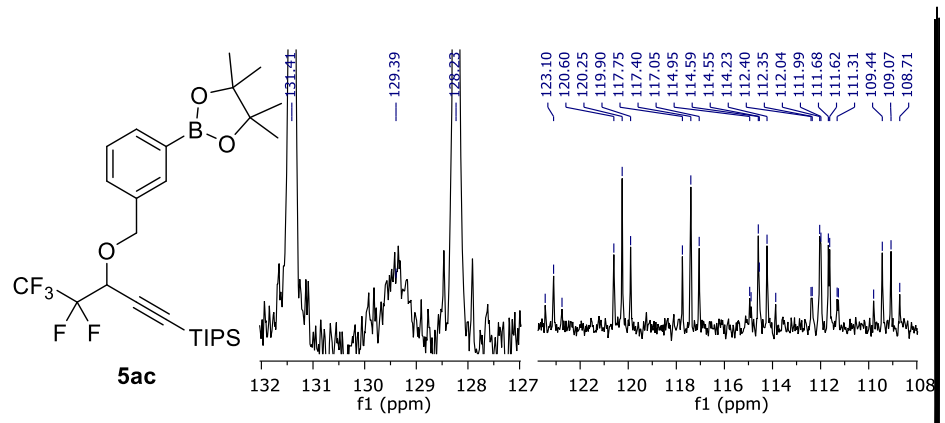

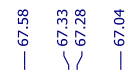

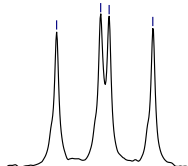

$\begin{array}{ll}130 & 129 \\ \mathrm{f} 1(\mathrm{ppm})\end{array}$ 
${ }^{19}$ F-NMR (376 MHz, $\mathrm{CDCl}_{3}$ ) of compound 5 ac

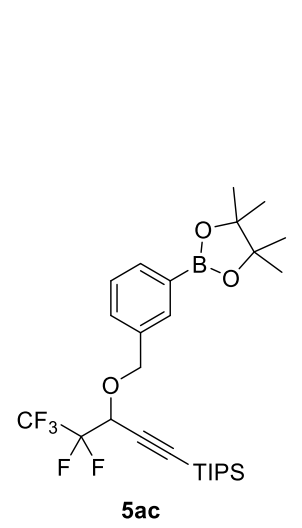

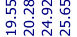

पर

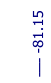

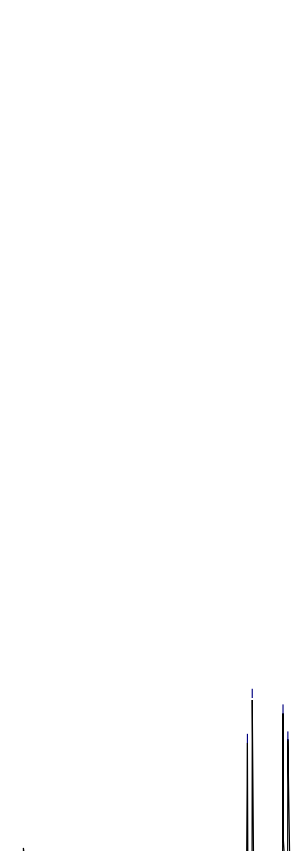

10

$-90 \underset{f 1(p p m)}{-100}-11$

$\begin{array}{llllllllllll}10 & -120 & -130 & -140 & -150 & -160 & -170 & -180 & -190 & -200 & -210\end{array}$ 
${ }^{1} \mathrm{H}-\mathrm{NMR}\left(400 \mathrm{MHz}, \mathrm{CDCl}_{3}\right.$ ) of compound $\mathbf{5 a d}$

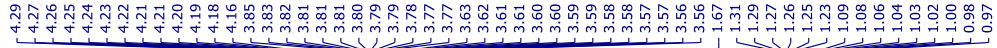
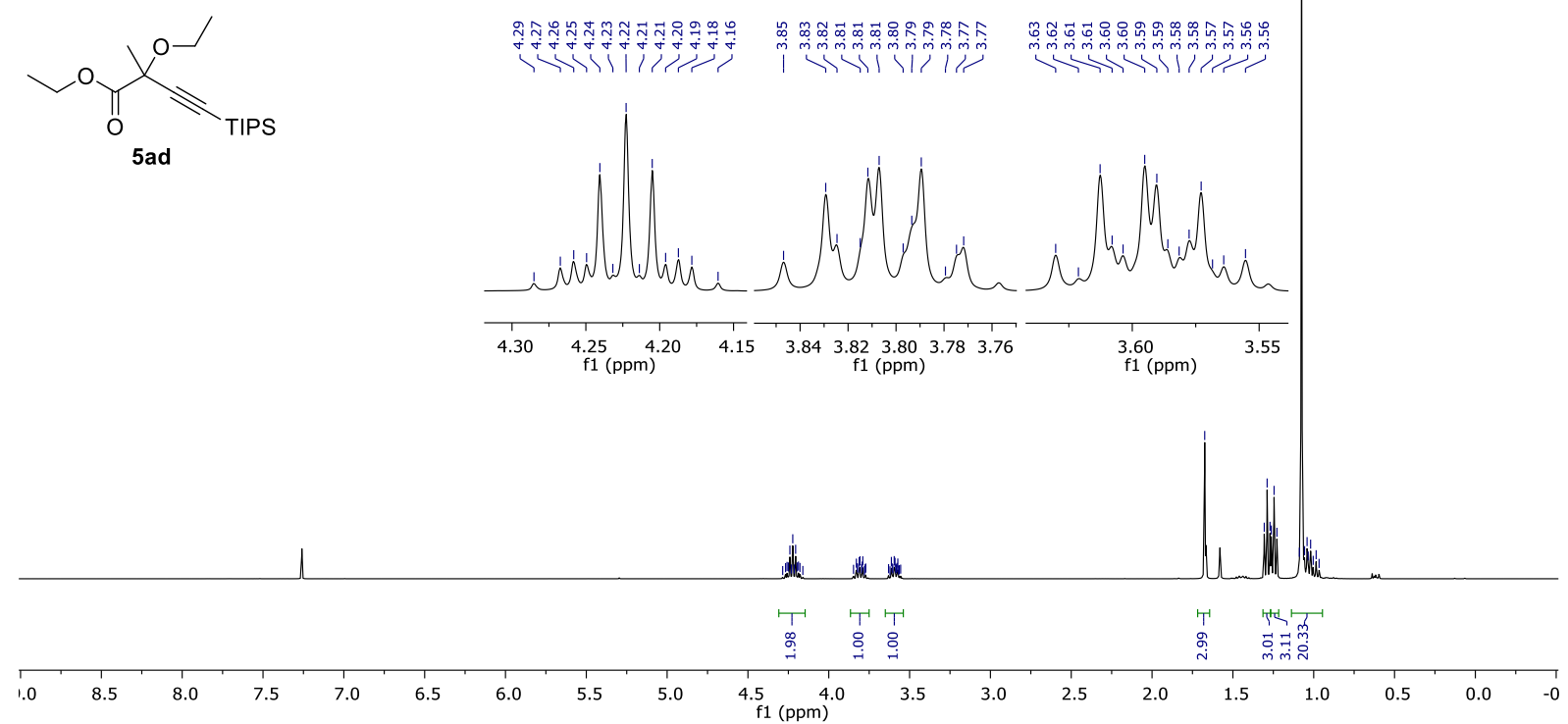

${ }^{13} \mathrm{C}$-NMR (101 MHz, $\mathrm{CDCl}_{3}$ ) of compound 5 ad

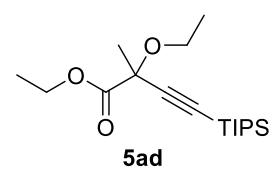
年

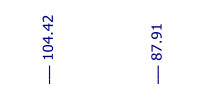

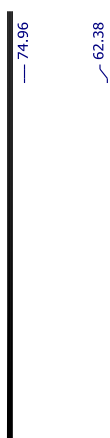

|

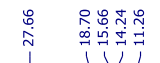

i 11,1

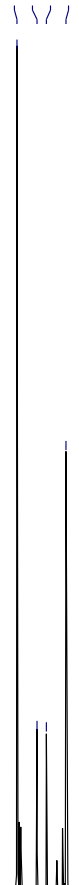

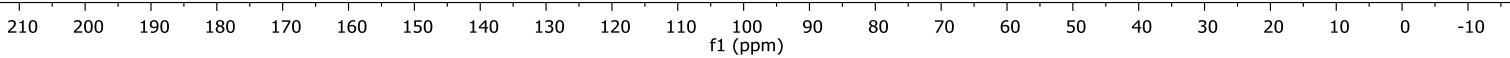


${ }^{1} \mathrm{H}-\mathrm{NMR}\left(400 \mathrm{MHz}, \mathrm{CDCl}_{3}\right.$ ) of compound 5 ae

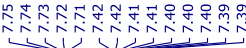

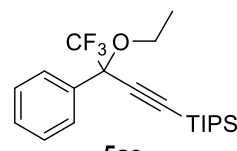

$5 a{ }^{2}$

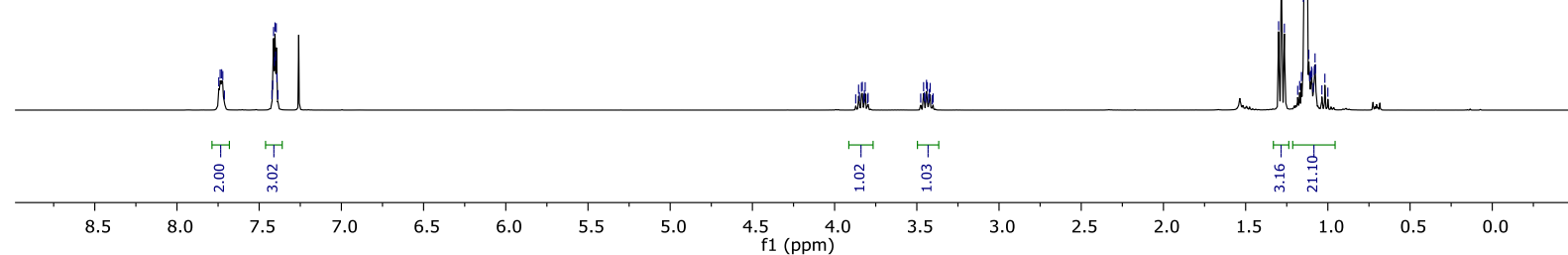

${ }^{13} \mathrm{C}-\mathrm{NMR}\left(101 \mathrm{MHz}, \mathrm{CDCl}_{3}\right)$ of compound 5ae

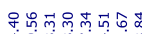

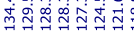

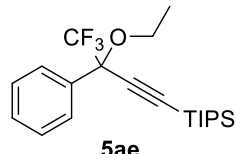

$5 \mathrm{ae}$

IPS
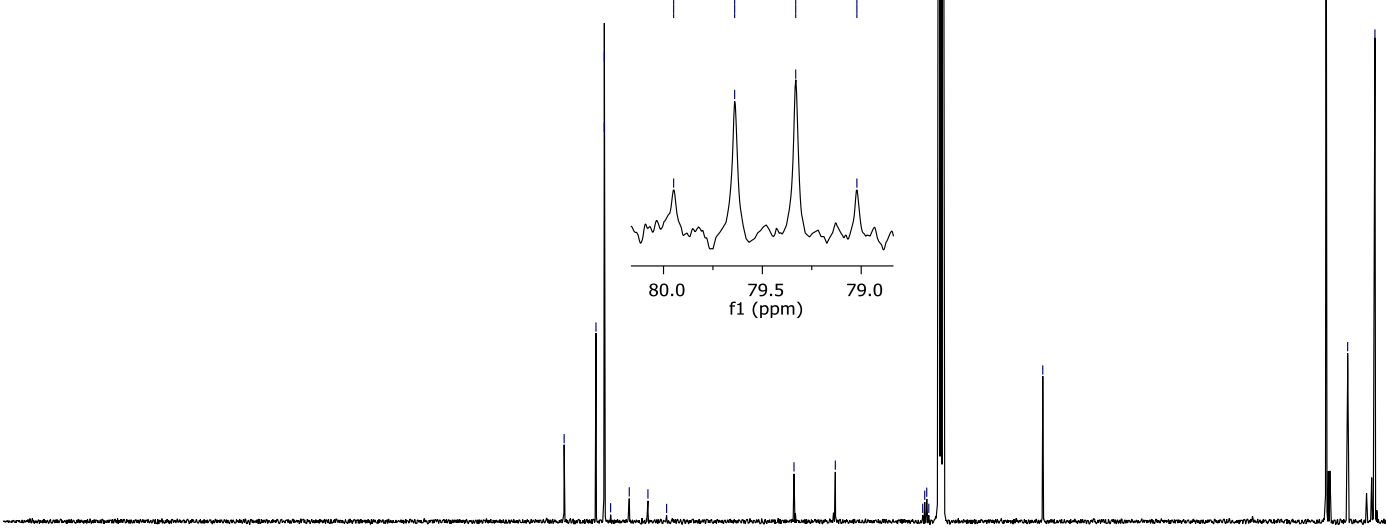

$\begin{array}{lllllllllll}210 & 200 & 190 & 180 & 170 & 160 & 150 & 140 & 130 & 120 & 110 \underset{\mathrm{f} 1(\mathrm{ppm})}{100} 90\end{array}$ 
${ }^{19}$ F-NMR (376 MHz, $\mathrm{CDCl}_{3}$ ) of compound 5 ae

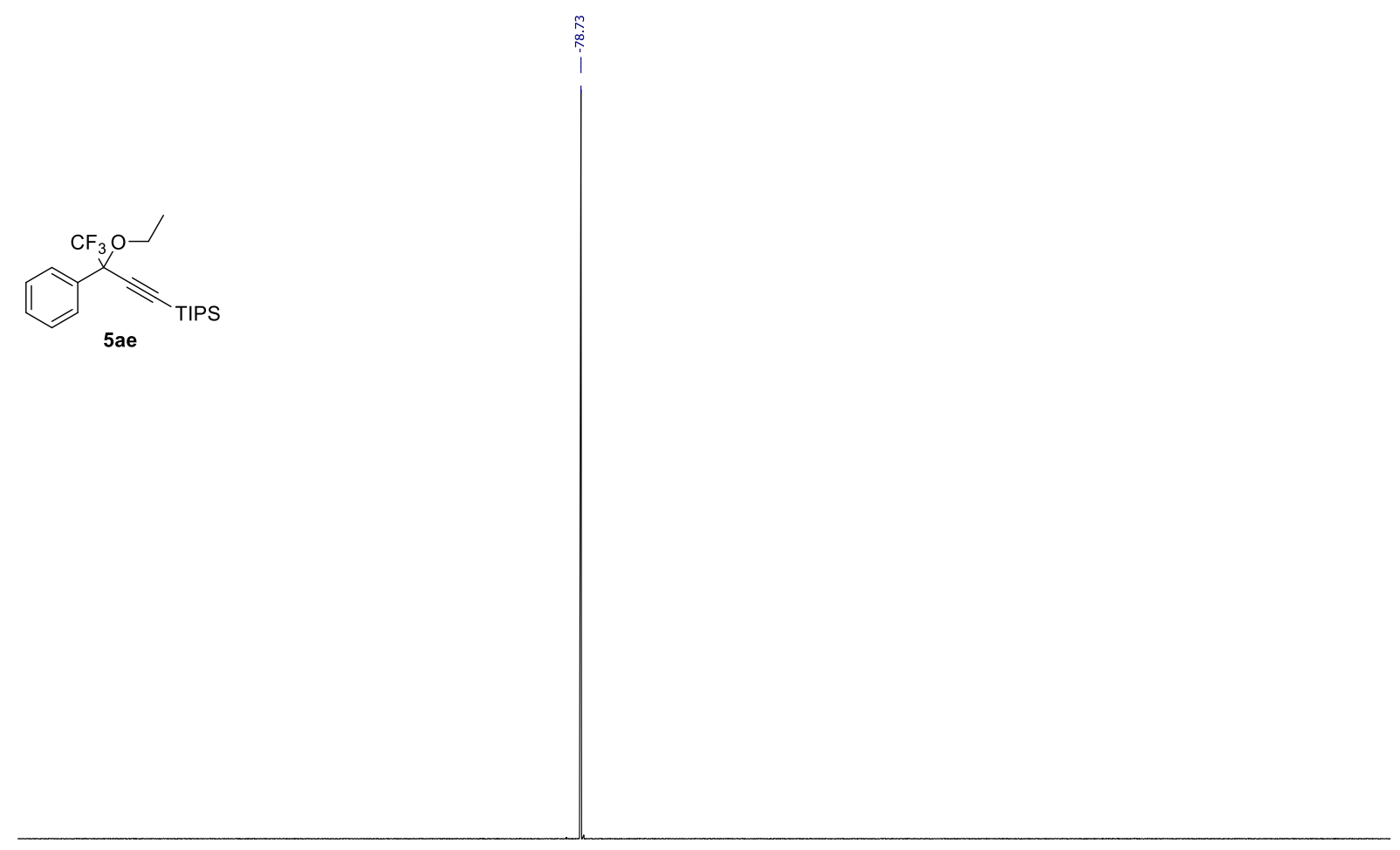

$\begin{array}{lllllllllllllllllllllllllllllll}10 & 0 & -10 & -20 & -30 & -40 & -50 & -60 & -70 & -80 & -90 & -100 & -110 & -120 & -130 & -140 & -150 & -160 & -170 & -180 & -190 & -200 & -210\end{array}$ 

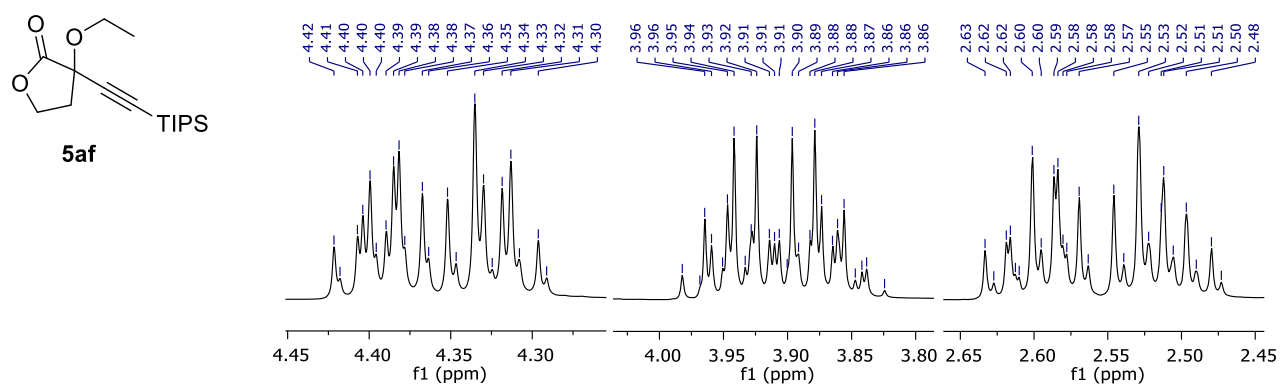

5af

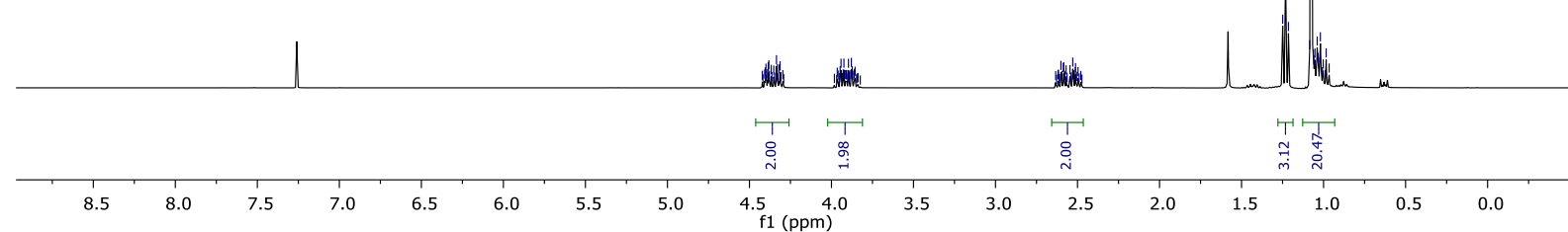

${ }^{13} \mathrm{C}$-NMR (101 MHz, $\mathrm{CDCl}_{3}$ ) of compound 5af

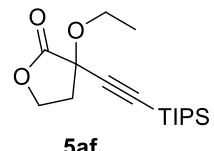

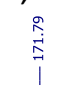

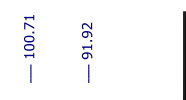

|

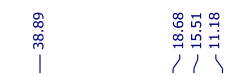

\footnotetext{
.
}


${ }^{1}$ H-NMR $\left(400 \mathrm{MHz}, \mathrm{CDCl}_{3}\right)$ of compound $5 \mathrm{ag}$

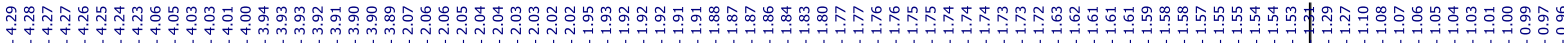

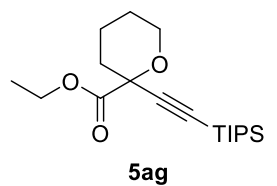

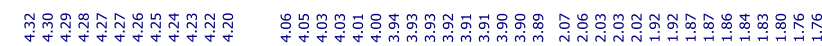
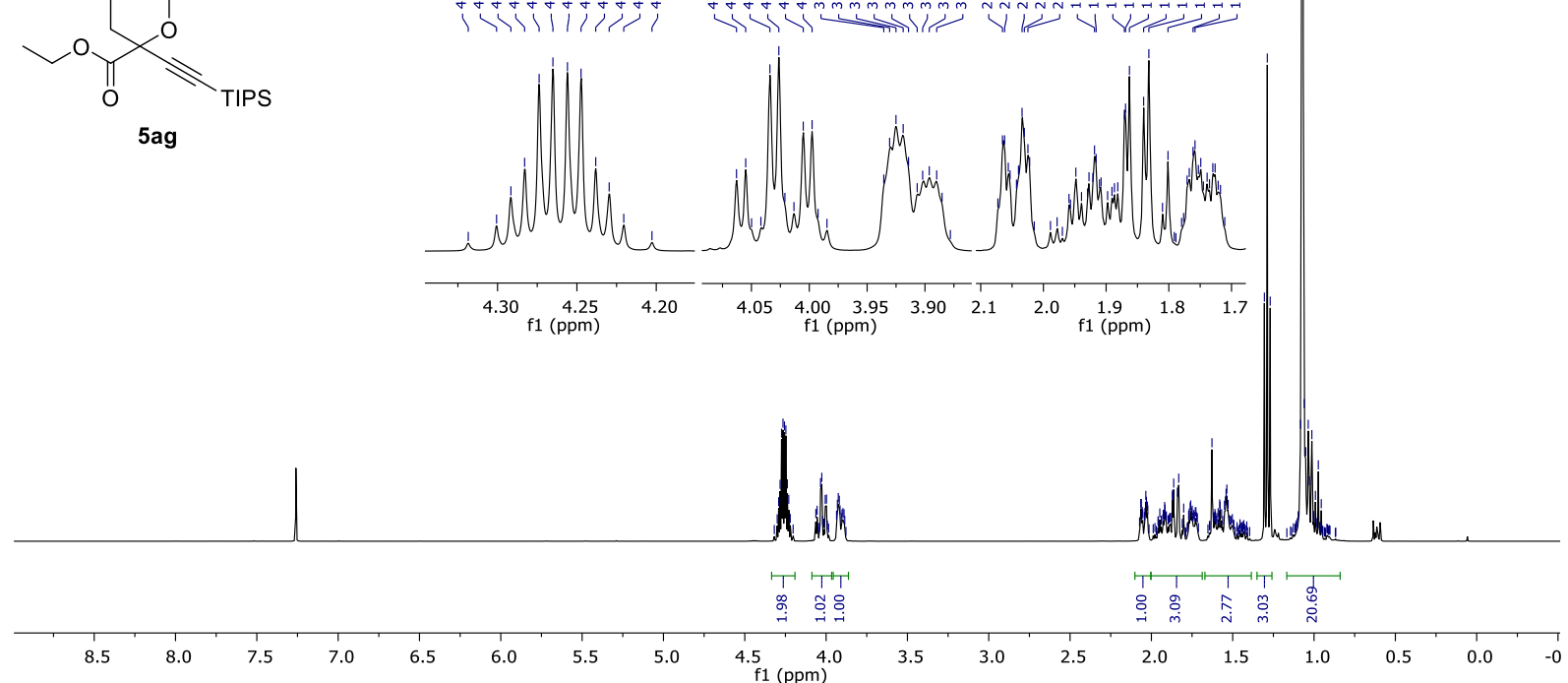

${ }^{13} \mathrm{C}-\mathrm{NMR}\left(101 \mathrm{MHz}, \mathrm{CDCl}_{3}\right)$ of compound $\mathbf{5 a g}$ i
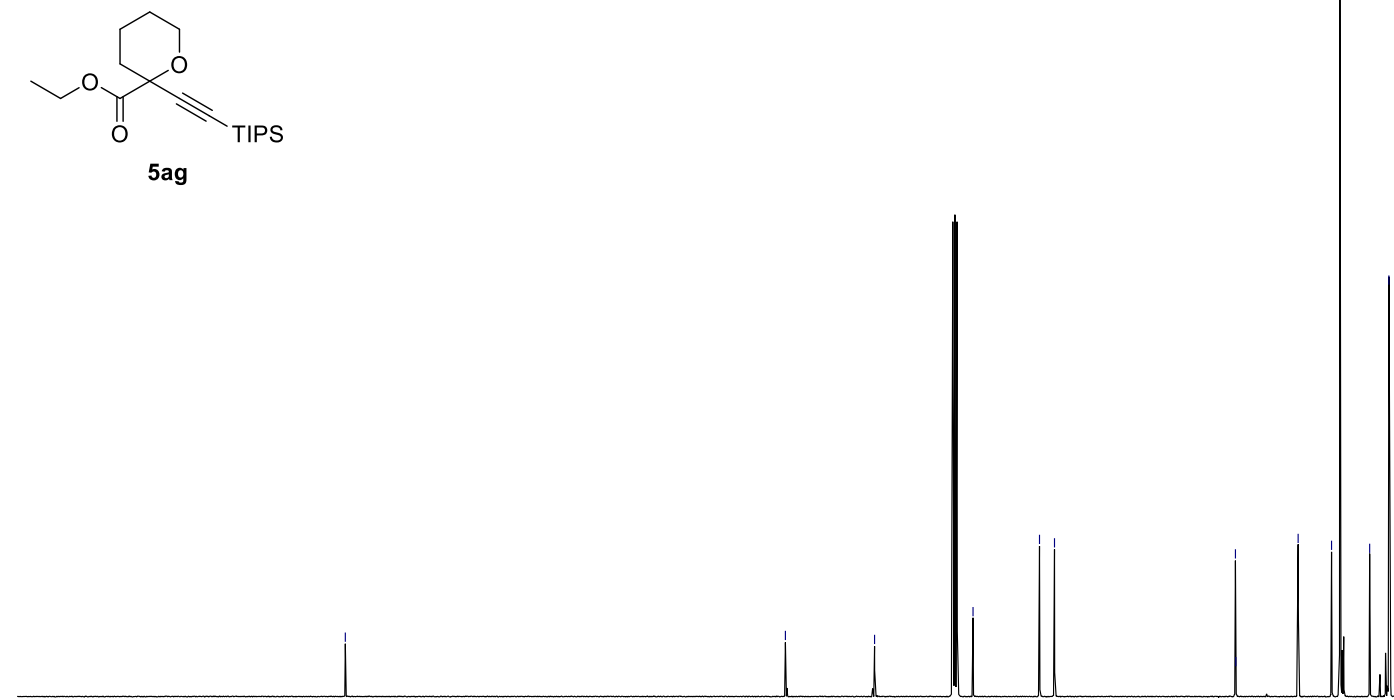

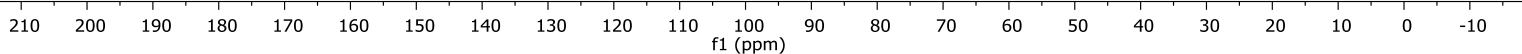


${ }^{1}$ H-NMR (400 MHz, $\mathrm{CDCl}_{3}$ ) of compound 5ai

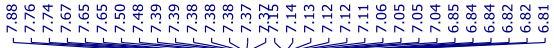

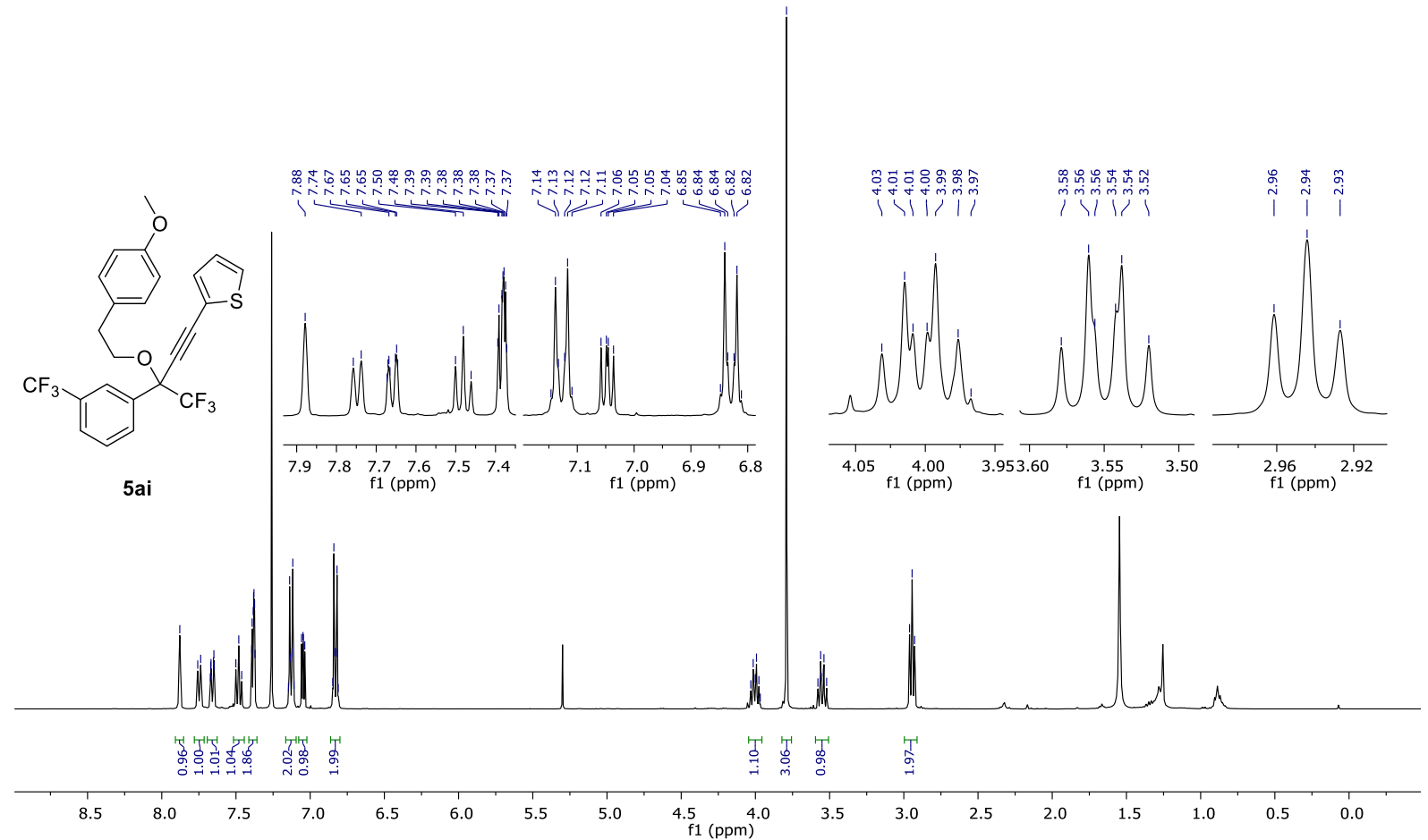

${ }^{13} \mathrm{C}$-NMR $\left(101 \mathrm{MHz}, \mathrm{CDCl}_{3}\right)$ of compound 5 ai

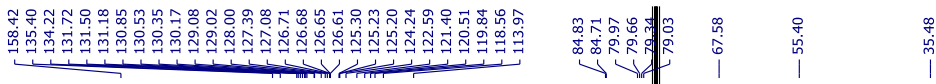

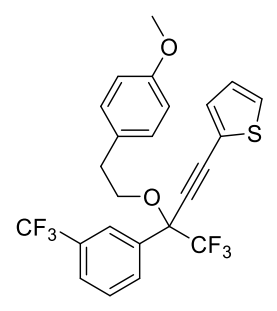

5ai

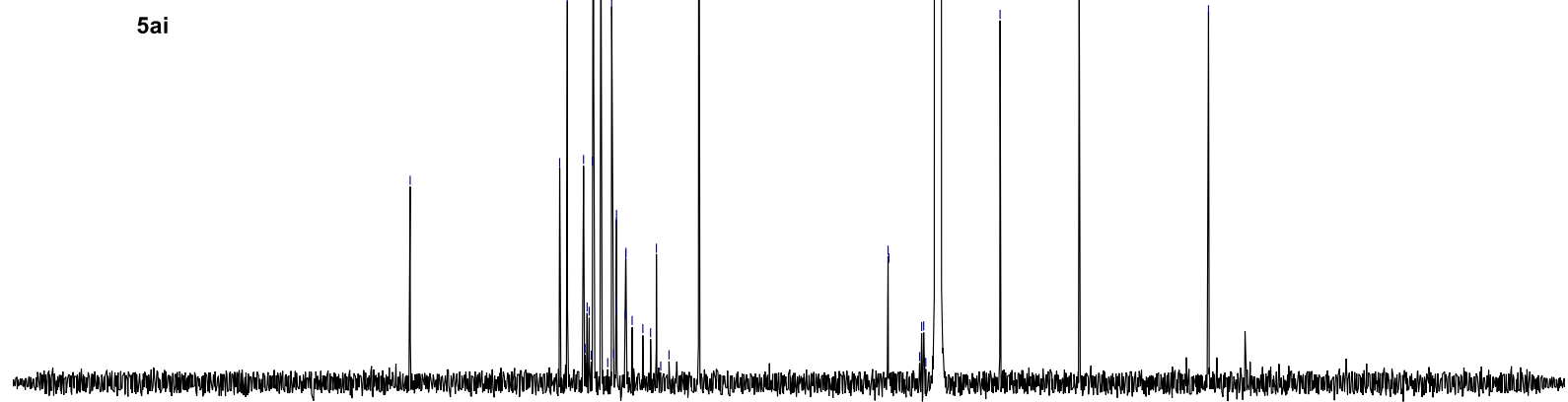

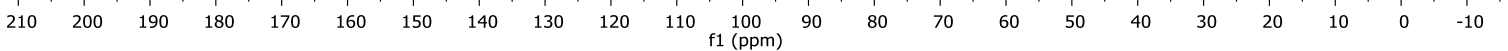


${ }^{19}$ F-NMR (376 MHz, $\mathrm{CDCl}_{3}$ ) of compound 5ai

$\stackrel{\substack{+0}}{\substack{n \\ 0}}$
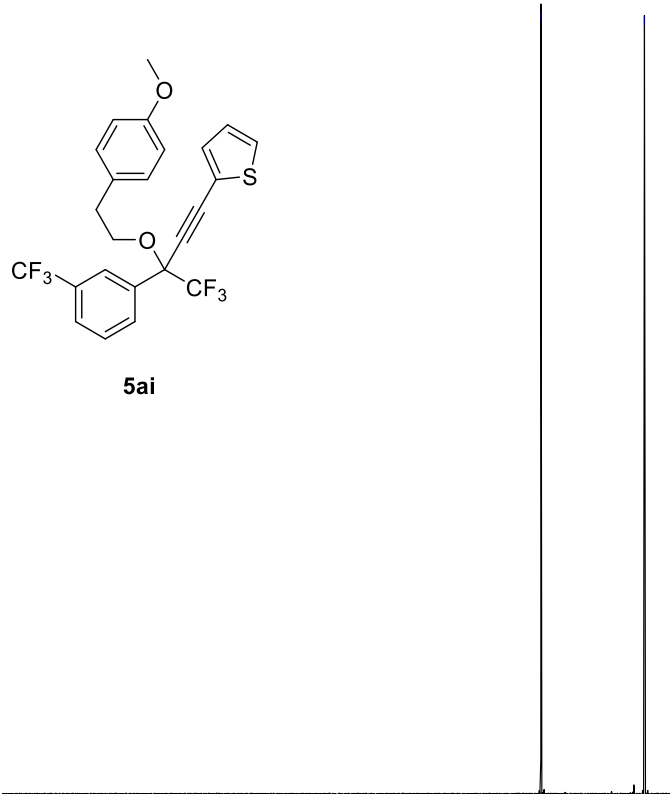

$\begin{array}{lllllllllllllllllllllllll}10 & 0 & -10 & -20 & -30 & -40 & -50 & -60 & -70 & -80 & -90 & -100 & -110 & -120 & -130 & -140 & -150 & -160 & -170 & -180 & -190 & -200 & -210\end{array}$ 


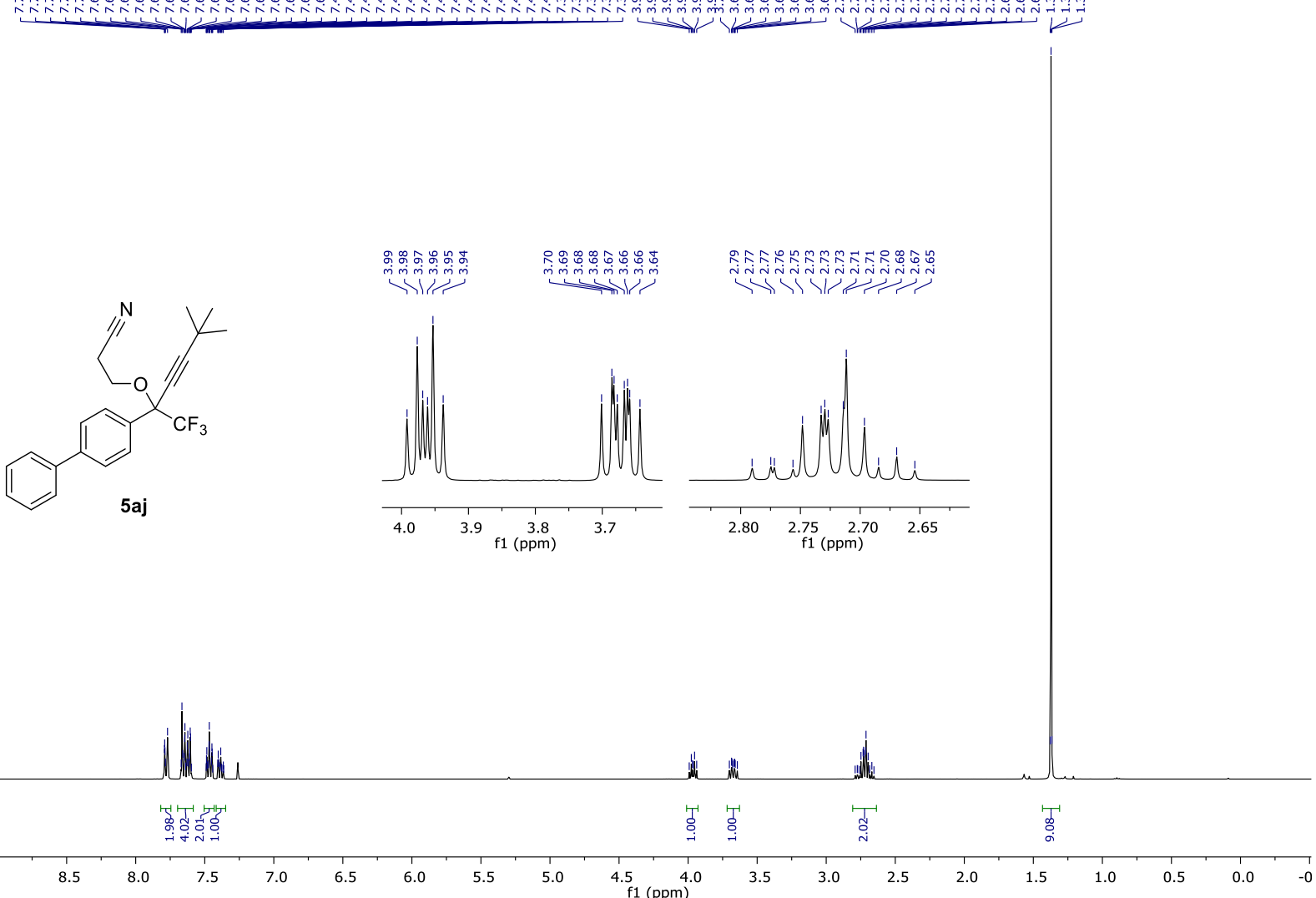

${ }^{13} \mathrm{C}-\mathrm{NMR}\left(101 \mathrm{MHz}, \mathrm{CDCl}_{3}\right)$ of compound 5aj

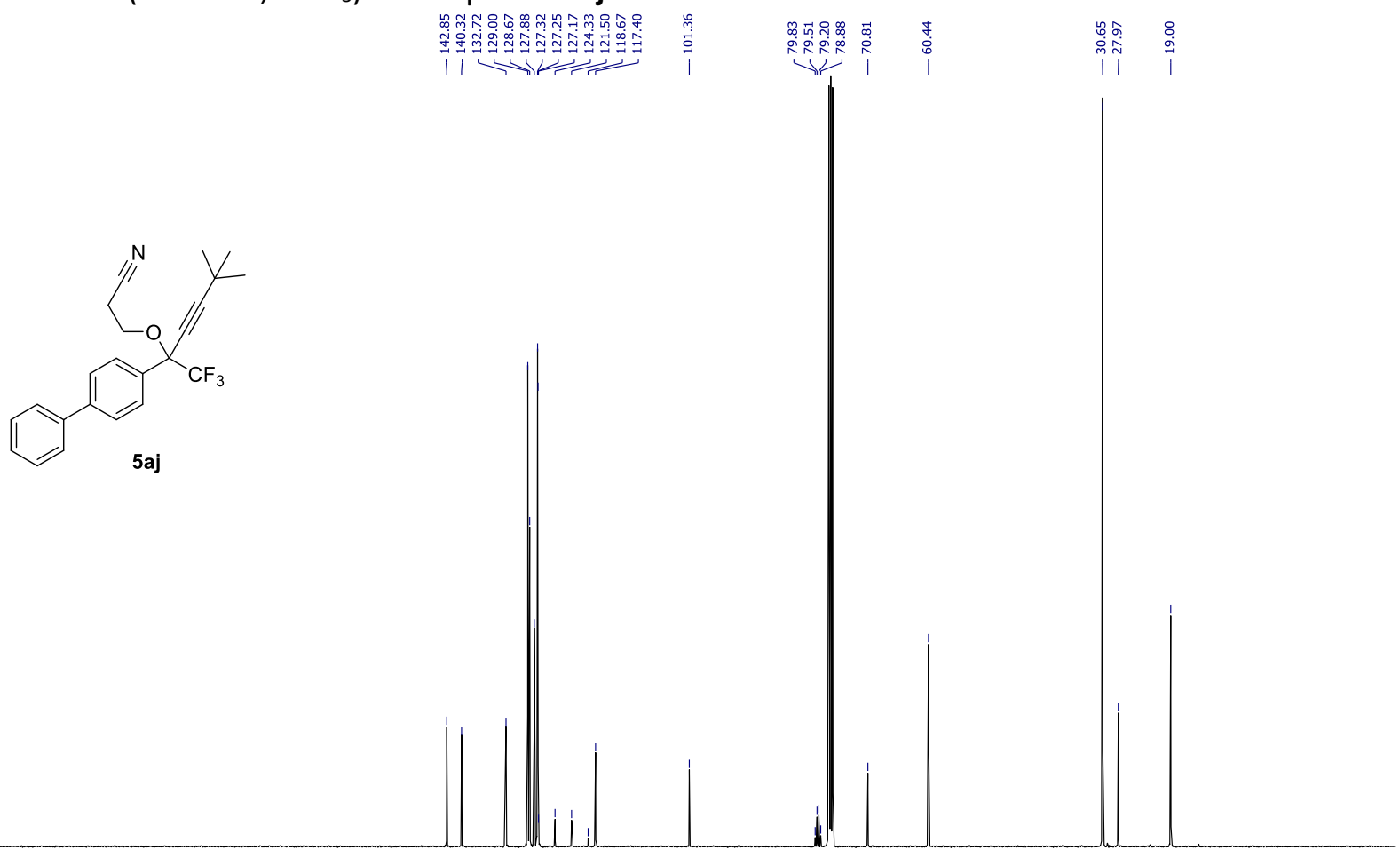

$\begin{array}{llllllllllllllllllllllllllll}210 & 200 & 190 & 180 & 170 & 160 & 150 & 140 & 130 & 120 & 110 & 100 & 90 & 80 & 70 & 60 & 50 & 40 & 30 & 20 & 10 & 0 & -10\end{array}$ 
${ }^{19}$ F-NMR (376 MHz, $\mathrm{CDCl}_{3}$ ) of compound 5aj

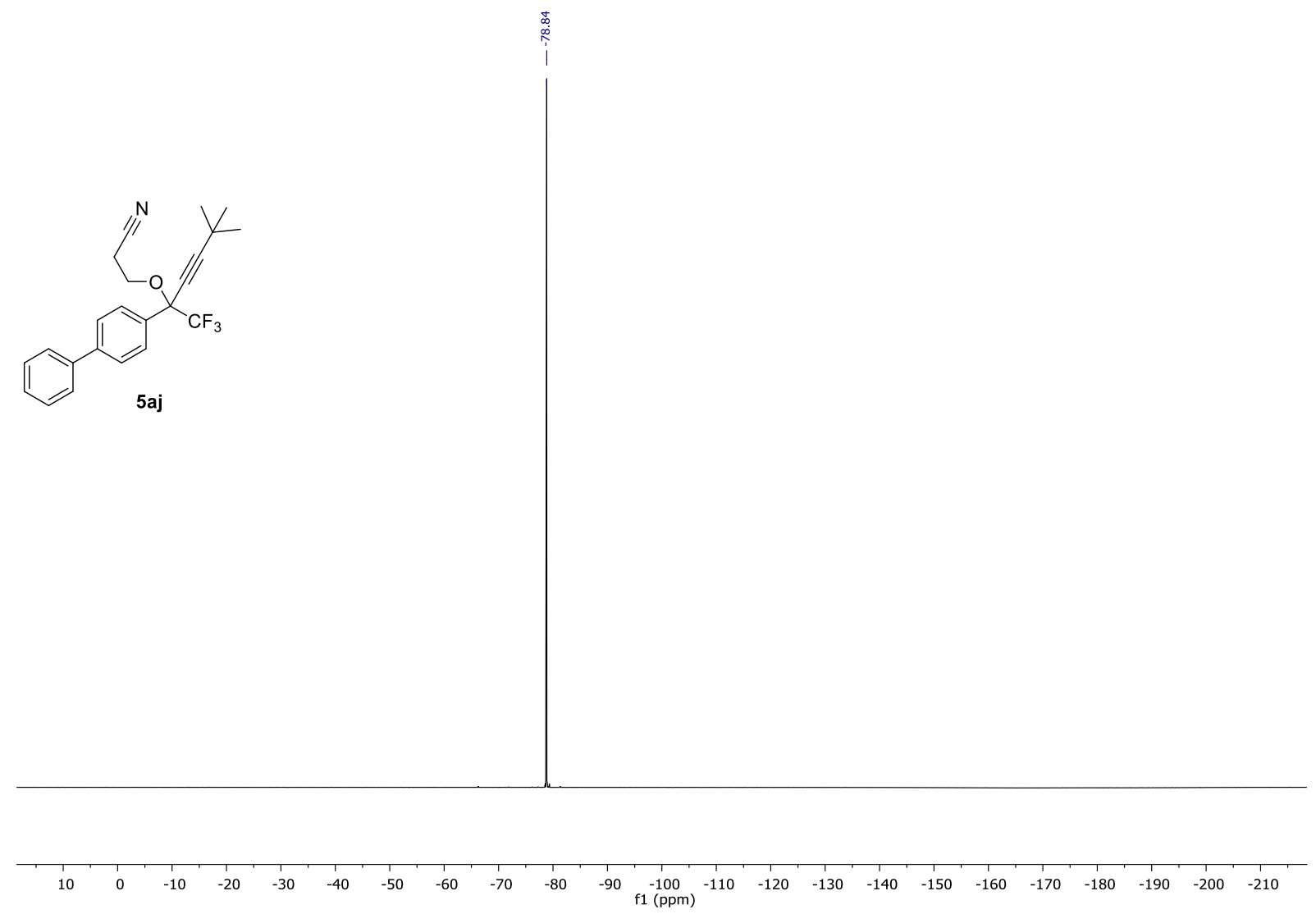


${ }^{1}$ H-NMR (400 MHz, $\mathrm{CDCl}_{3}$ ) of compound 5ak 等

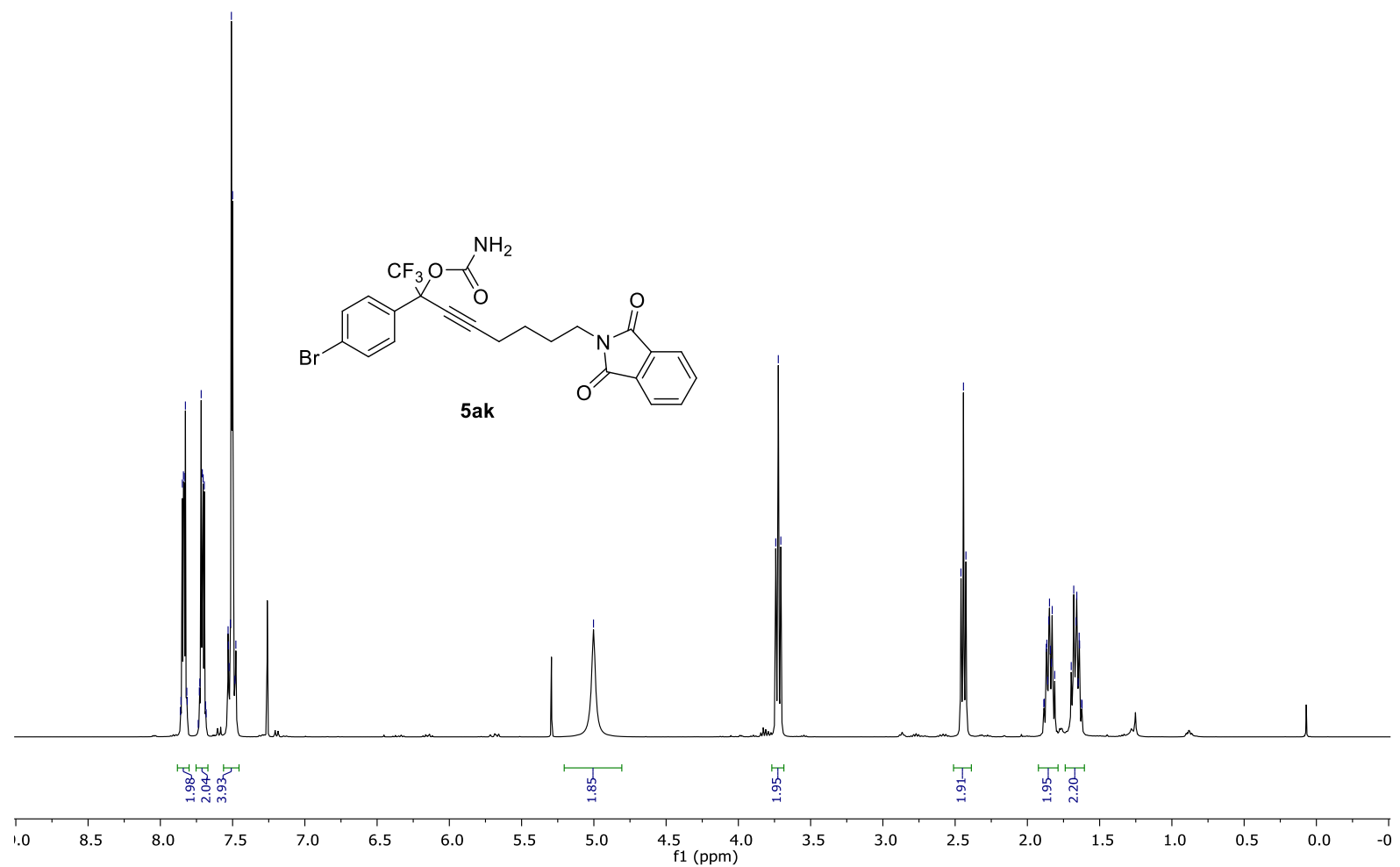

${ }^{13} \mathrm{C}$-NMR (101 MHz, $\mathrm{CDCl}_{3}$ ) of compound 5ak

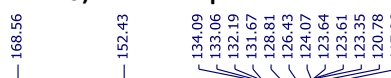<smiles>CC(C)(OC(N)=O)c1ccc(Br)cc1</smiles>
5 ak
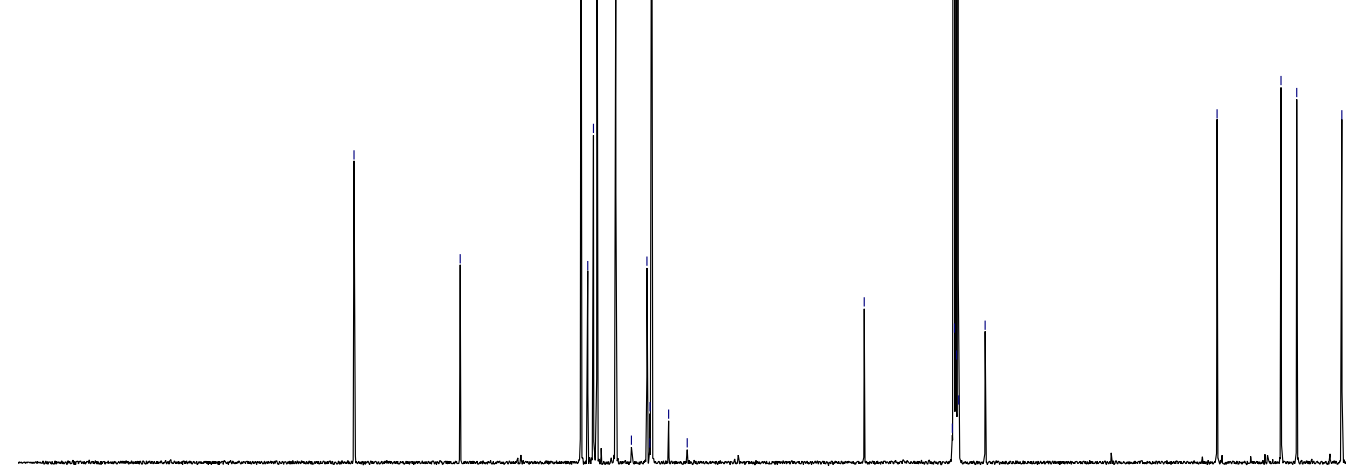
${ }^{19}$ F-NMR (376 MHz, $\mathrm{CDCl}_{3}$ ) of compound 5ak

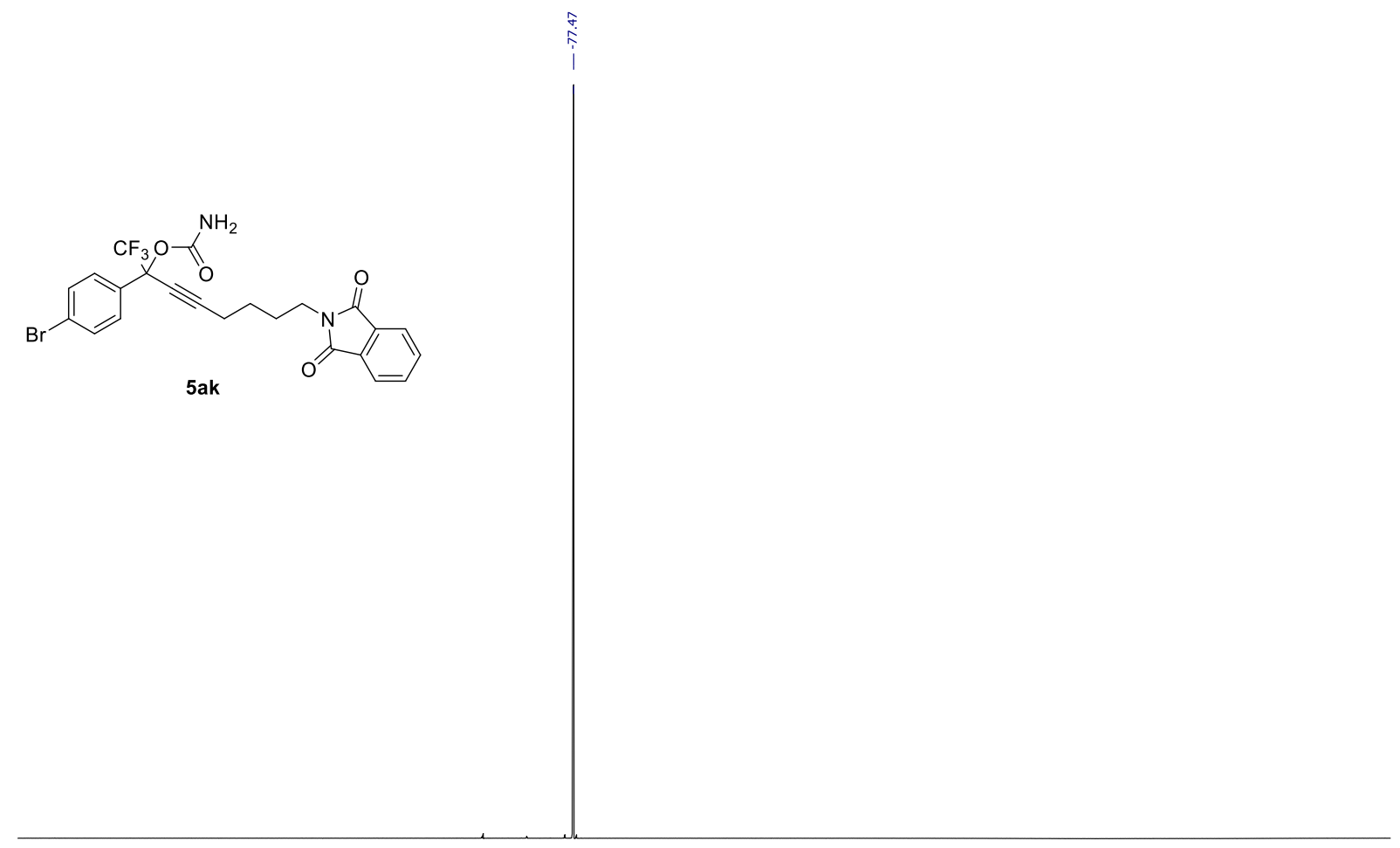

$\begin{array}{lllllllllllllllllllllllllll}10 & 0 & -10 & -20 & -30 & -40 & -50 & -60 & -70 & -80 & -90 & -100 & -110 & -120 & -130 & -140 & -150 & -160 & -170 & -180 & -190 & -200 & -210\end{array}$ 
${ }^{1} \mathrm{H}-\mathrm{NMR}\left(400 \mathrm{MHz}, \mathrm{CDCl}_{3}\right)$ of compound $\mathbf{3 C}$

等

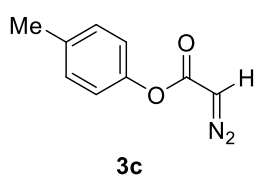

$3 c$

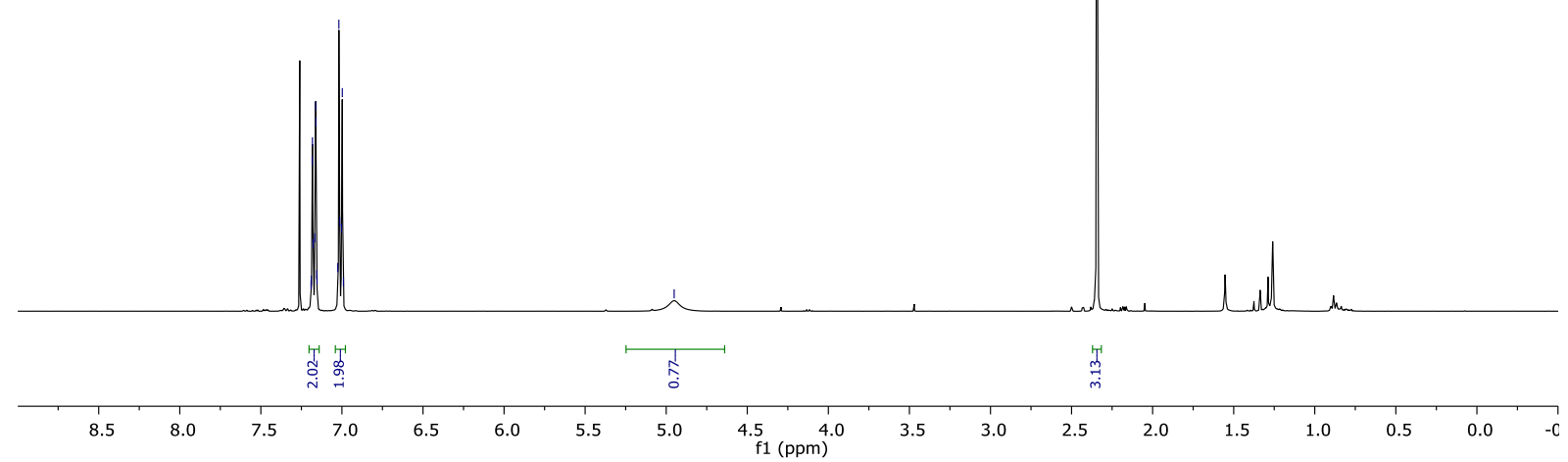

${ }^{13} \mathrm{C}$-NMR (101 MHz, $\mathrm{CDCl}_{3}$ ) of compound 3c

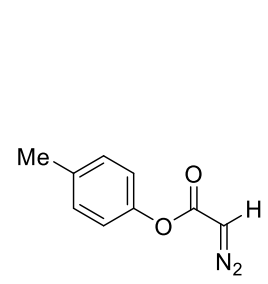

3c

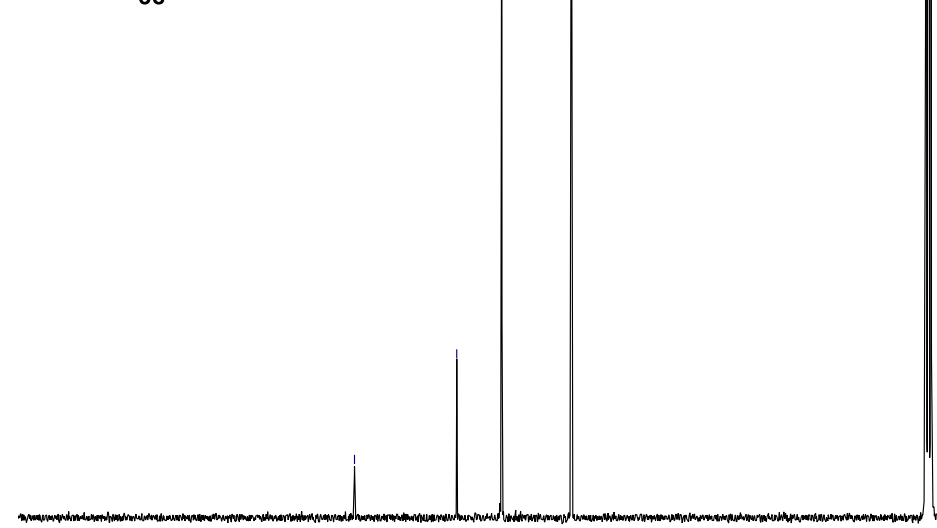

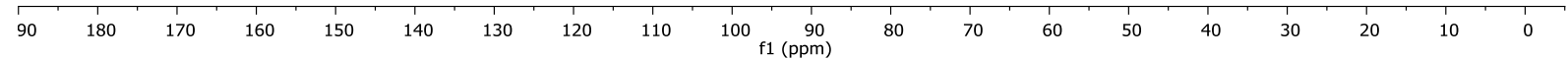


${ }^{1} \mathbf{H}-\mathrm{NMR}\left(400 \mathrm{MHz}, \mathrm{CDCl}_{3}\right)$ of compound $\mathbf{3 n}$

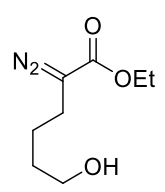

3n

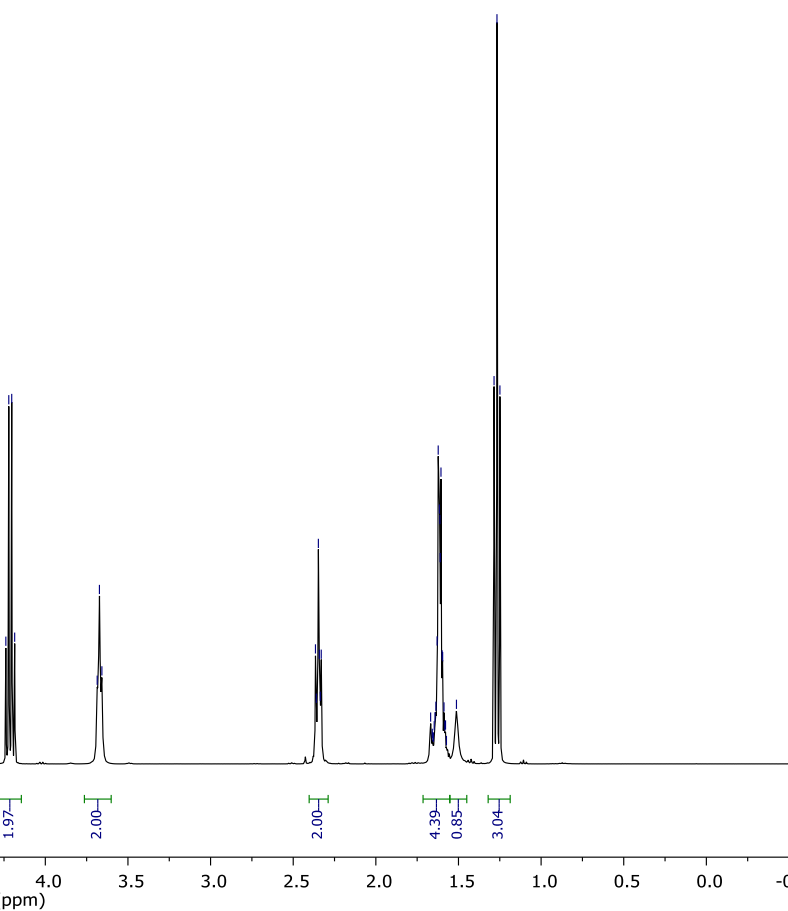

${ }^{13}$ C-NMR (101 MHz, $\mathrm{CDCl}_{3}$ ) of compound 3n

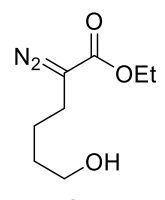

$3 n$ 
${ }^{1}$ H-NMR (400 MHz, $\mathrm{CDCl}_{3}$ ) of compound 30

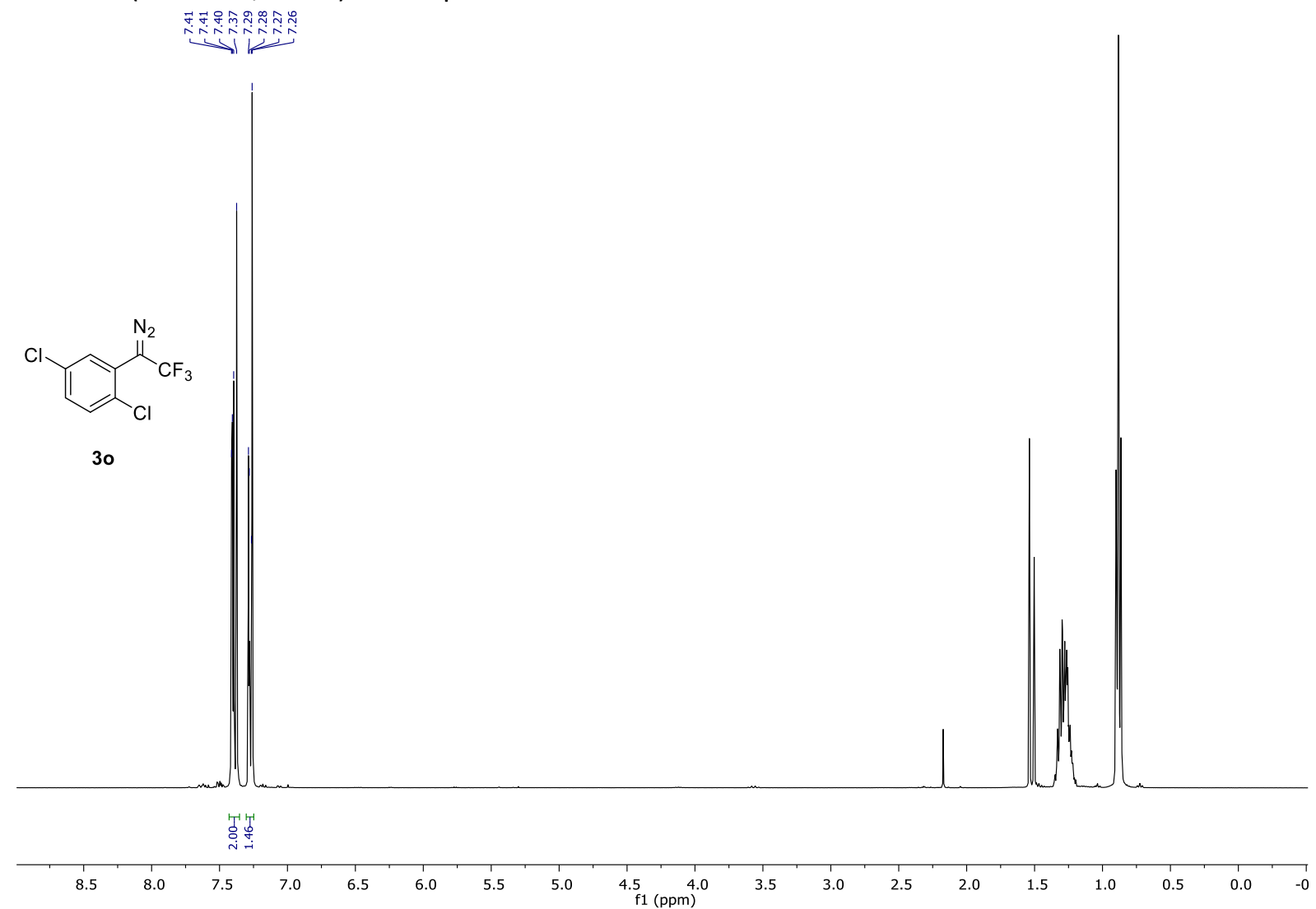

${ }^{13}$ C-NMR (101 MHz, $\mathrm{CDCl}_{3}$ ) of compound 30

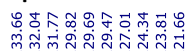

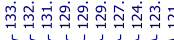

$\mathrm{Cl}_{\mathrm{CF}_{3}}^{\mathrm{N}_{2}}$

30

(1)

$\begin{array}{lllllllllll}210 & 200 & 190 & 180 & 170 & 160 & 150 & 140 & 130 & 120 & 110 \\ \mathrm{f} 1(\mathrm{ppm}) & 900\end{array}$

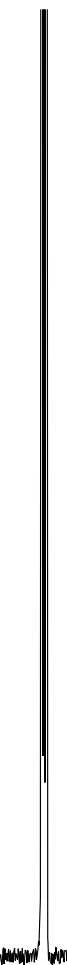


${ }^{19}$ F-NMR (376 MHz, $\mathrm{CDCl}_{3}$ ) of compound 30

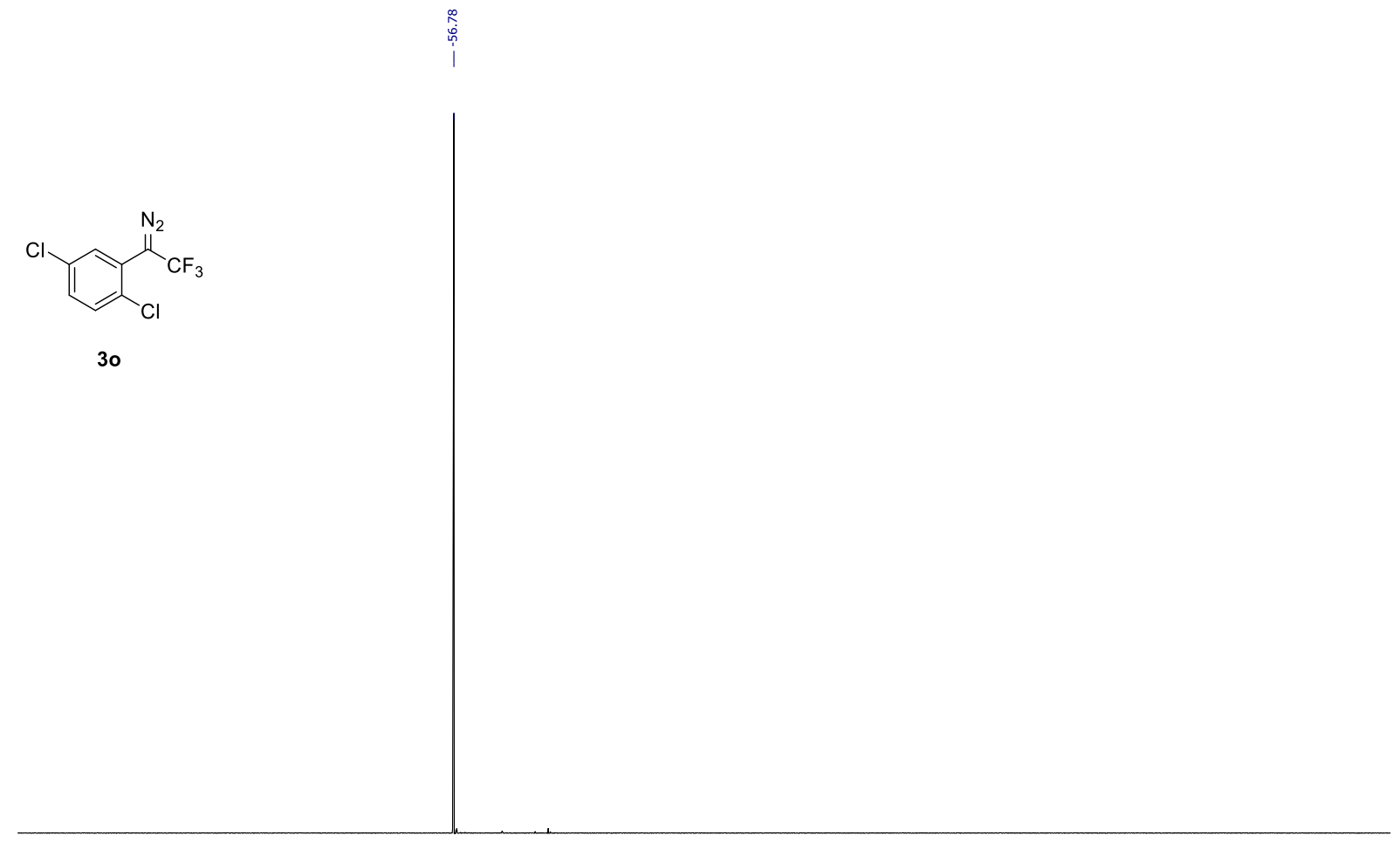

$\begin{array}{lllllllllllllllllllllllllllllll}10 & 0 & -10 & -20 & -30 & -40 & -50 & -60 & -70 & -80 & -90 & -100 & -110 & -120 & -130 & -140 & -150 & -160 & -170 & -180 & -190 & -200 & -210\end{array}$ 
${ }^{1} \mathrm{H}-\mathrm{NMR}\left(400 \mathrm{MHz}, \mathrm{CDCl}_{3}\right.$ ) of compound 3p

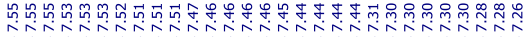

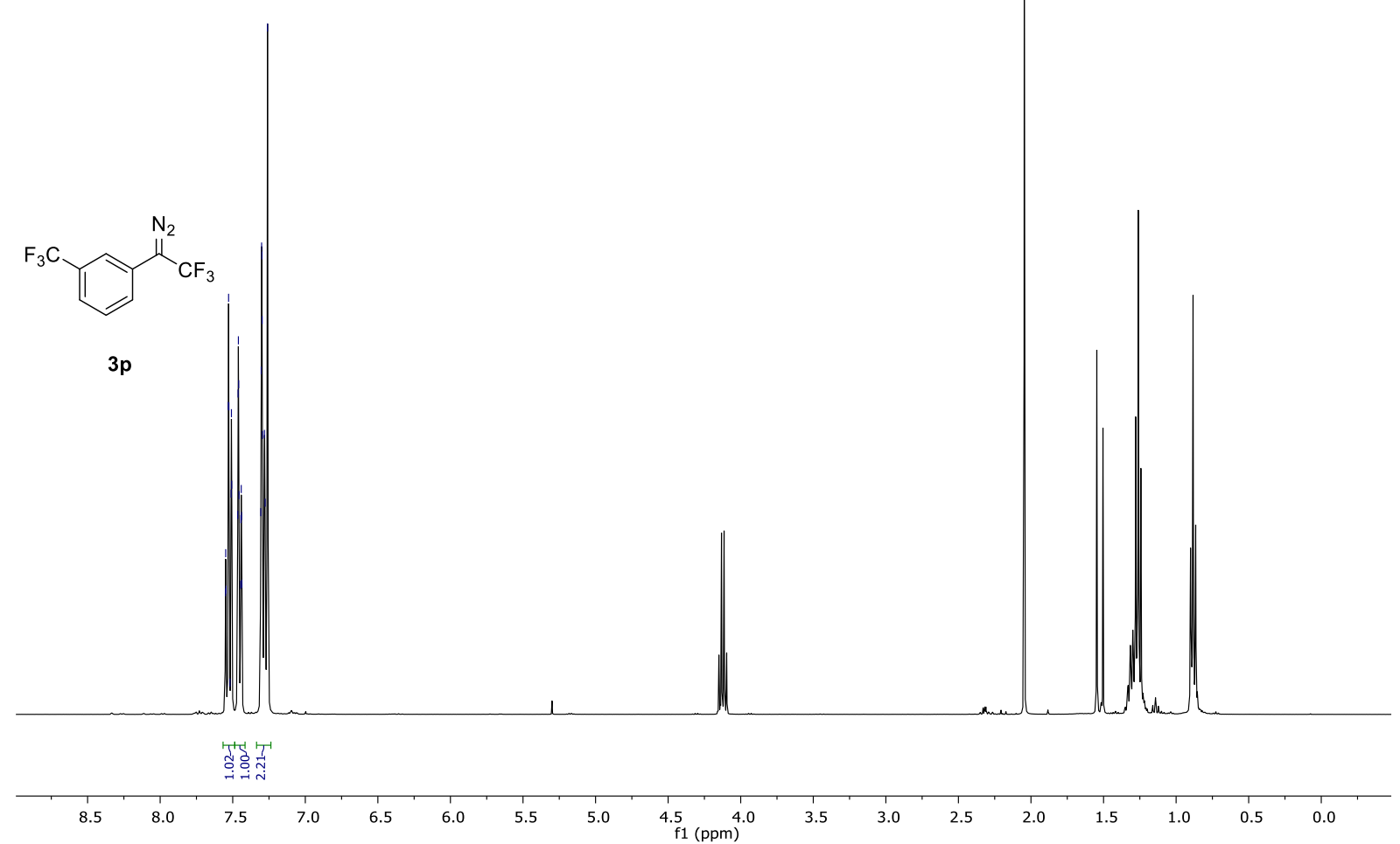

${ }^{13}$ C-NMR (101 MHz, $\mathrm{CDCl}_{3}$ ) of compound 3p

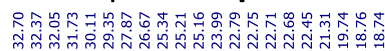

更

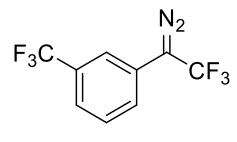

$3 p$

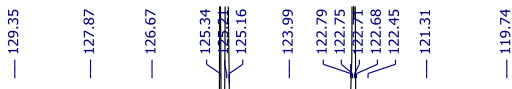
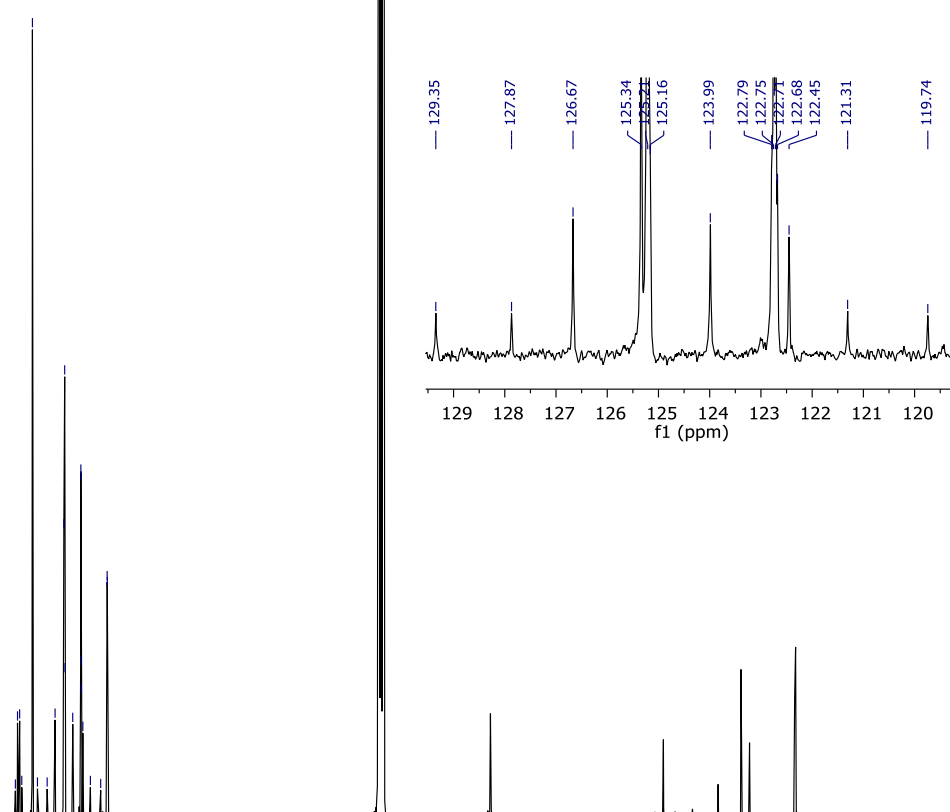

$\begin{array}{llllllllll}129 & 128 & 127 & 126 & \begin{array}{l}125 \\ \mathrm{f} 1(\mathrm{ppm})\end{array} & 123 & 122 & 121 & 120\end{array}$

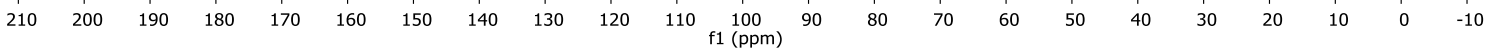


${ }^{19}$ F-NMR (376 MHz, $\mathrm{CDCl}_{3}$ ) of compound 3p

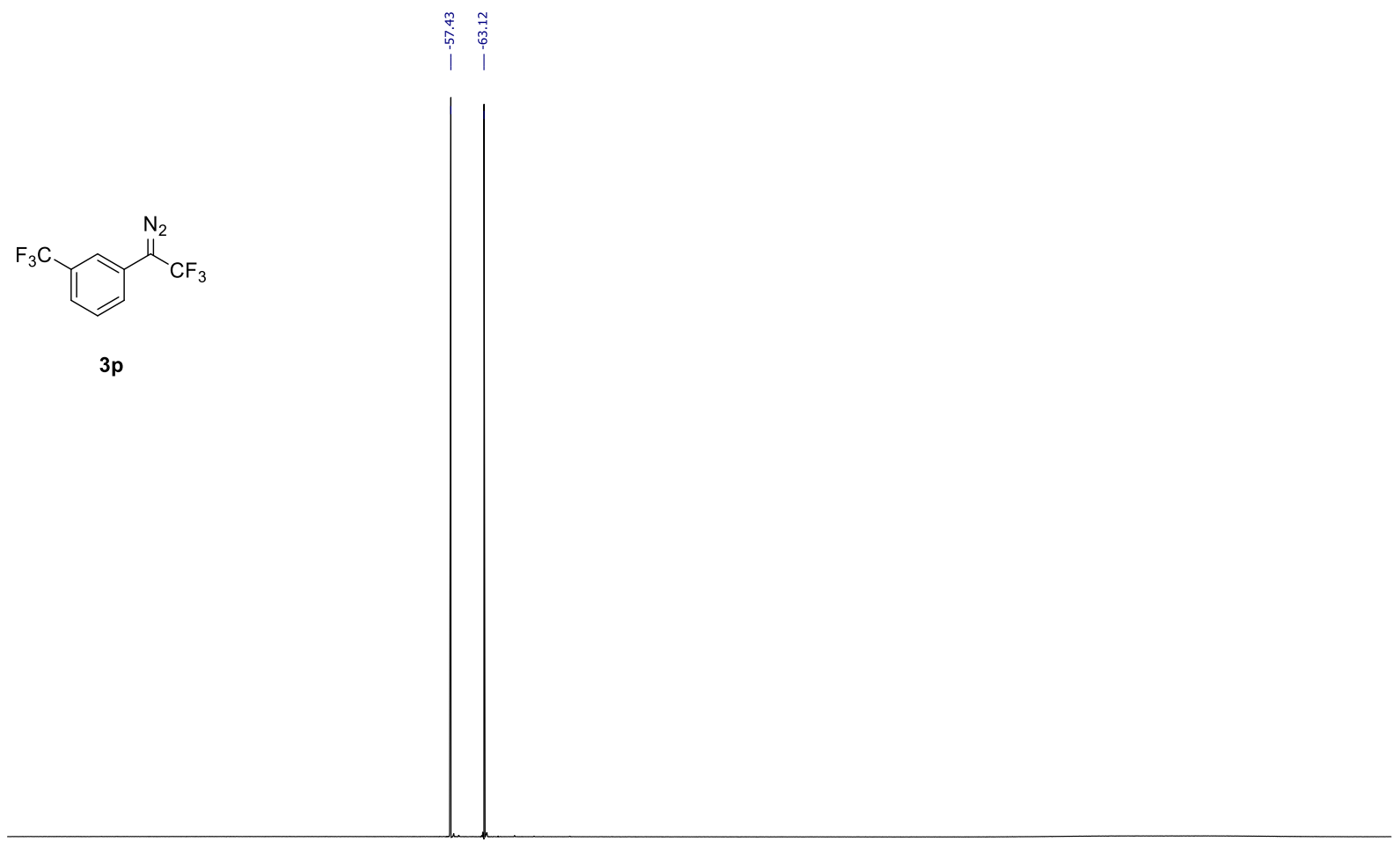

$\begin{array}{llllllllllllllllllllllllllll}10 & 0 & -10 & -20 & -30 & -40 & -50 & -60 & -70 & -80 & -90 & -100 & -110 & -120 & -130 & -140 & -150 & -160 & -170 & -180 & -190 & -200 & -210\end{array}$ 
${ }^{1} \mathbf{H}-\mathbf{N M R}\left(400 \mathrm{MHz}, \mathrm{CDCl}_{3}\right.$ ) of compound $\mathbf{2 b}$

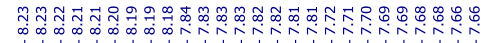

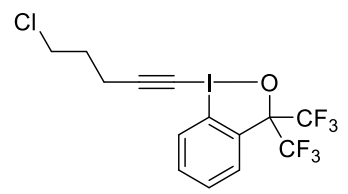

2b

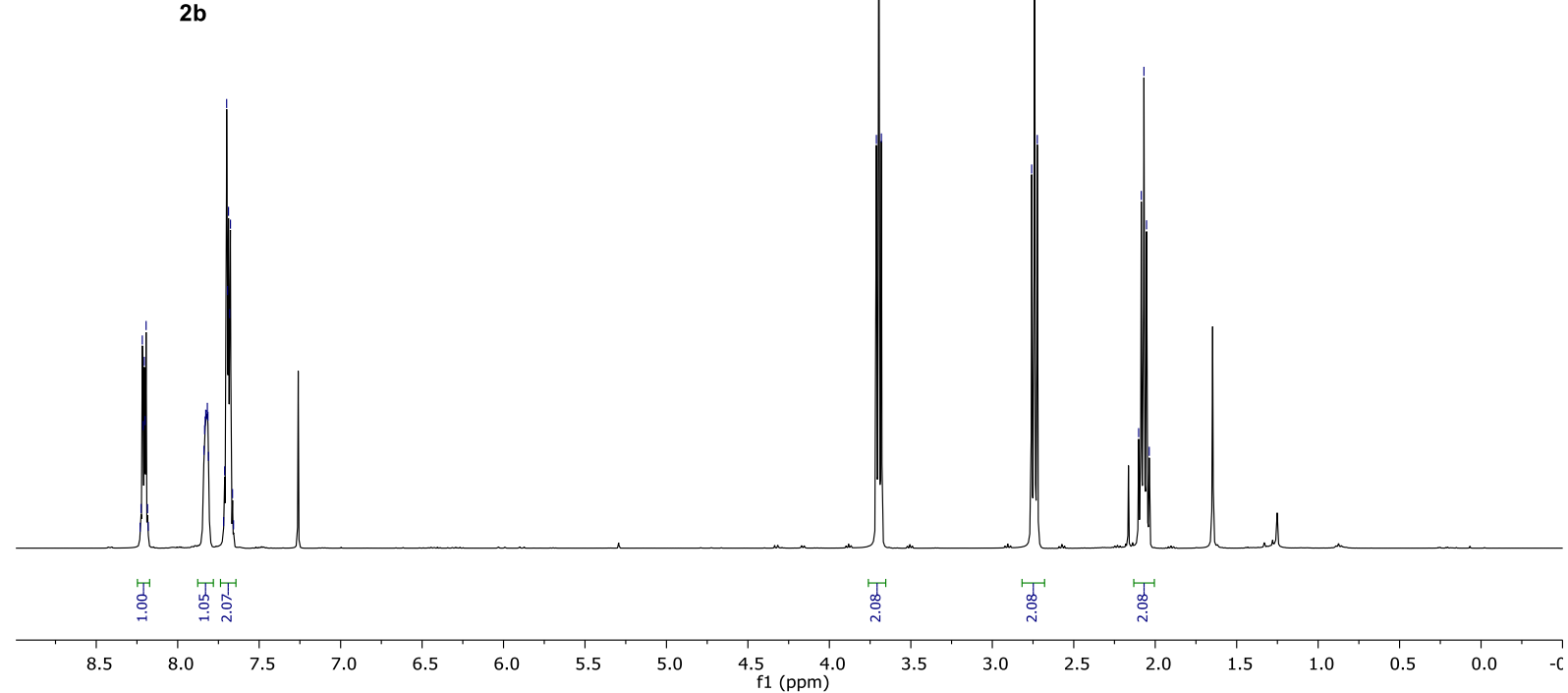

${ }^{13} \mathbf{C}$-NMR (101 MHz, $\mathrm{CDCl}_{3}$ ) of compound $\mathbf{2 b}$

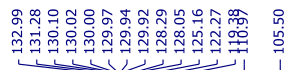

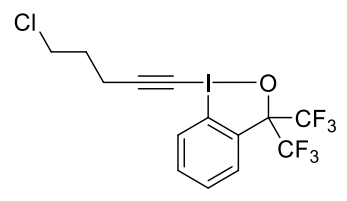

$2 \mathbf{b}$
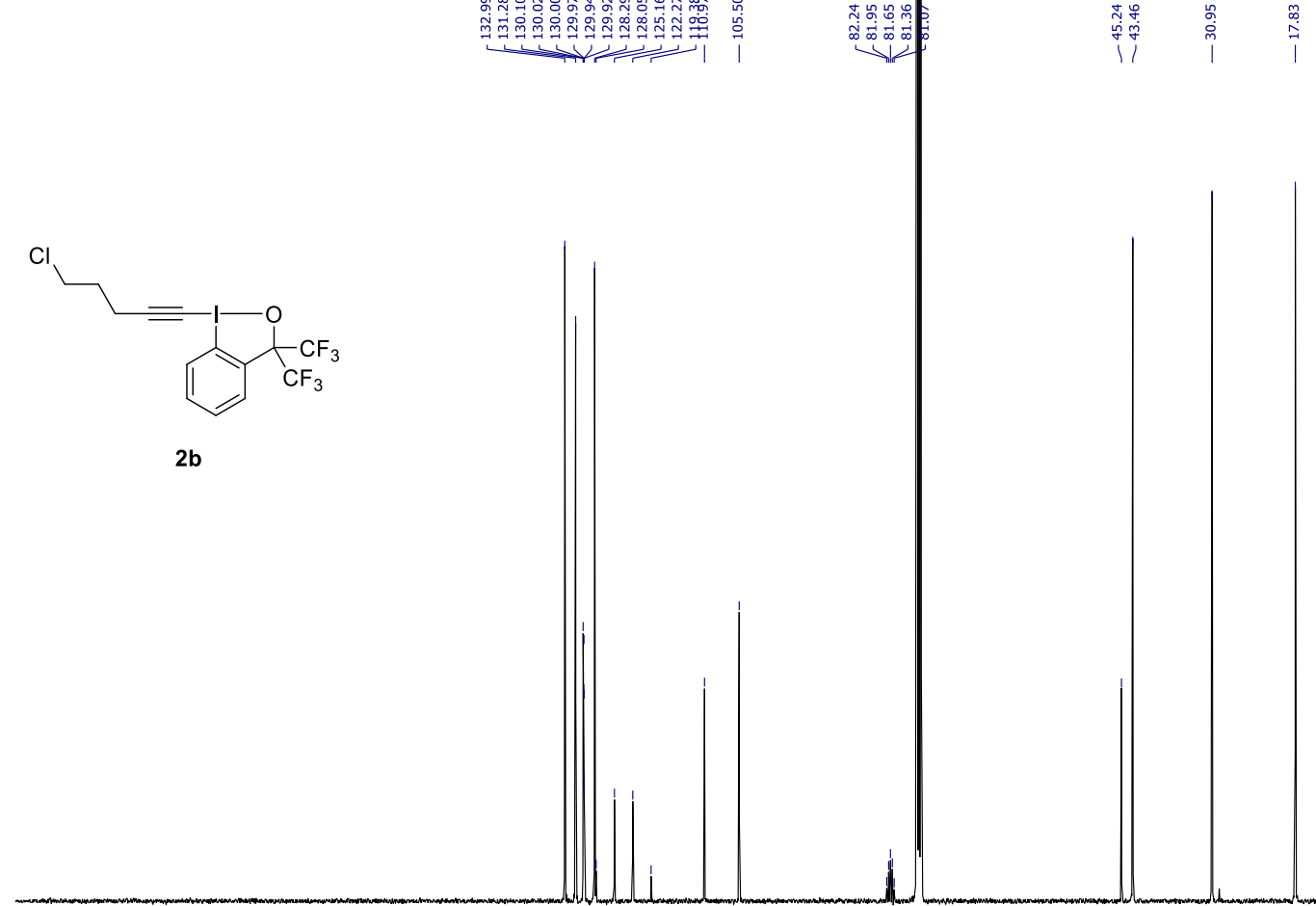

$\begin{array}{lllllllllll}210 & 200 & 190 & 180 & 170 & 160 & 150 & 140 & 130 & 120 & 110 \begin{array}{c}100 \\ \mathrm{f} 1(\mathrm{ppm})\end{array}\end{array}$ 
${ }^{19}$ F-NMR (376 MHz, CDCl $)$ of compound $\mathbf{2 b}$

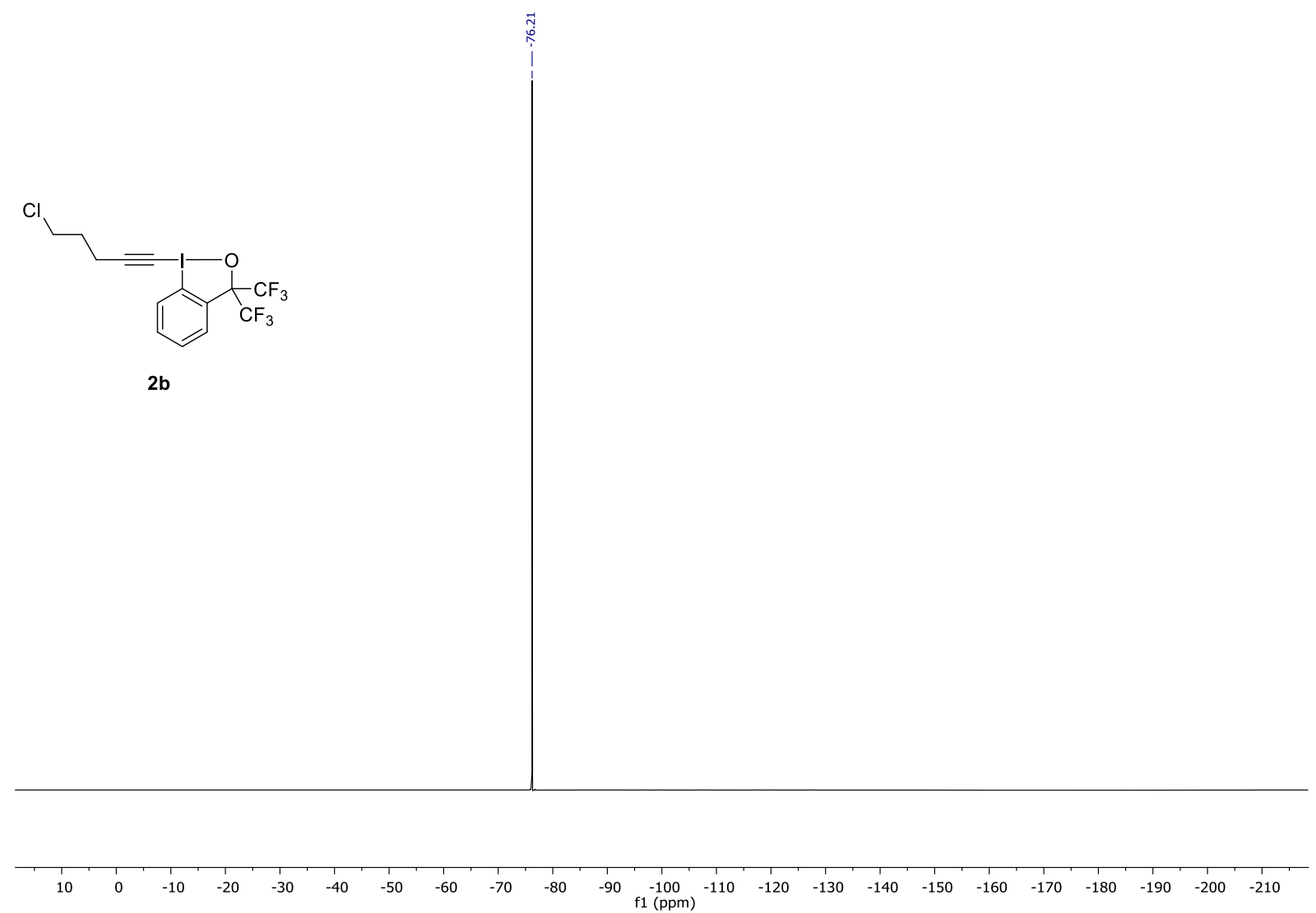




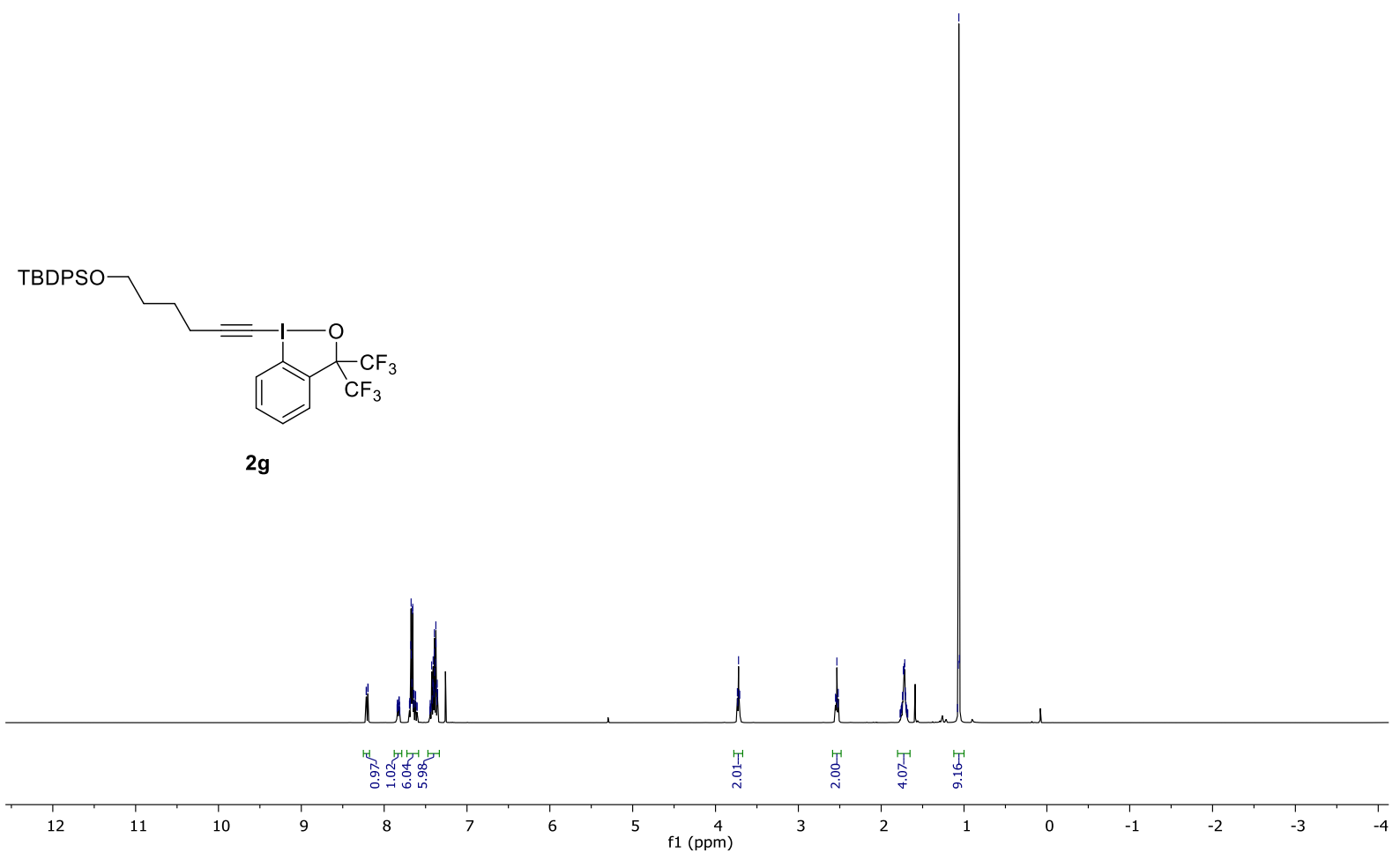

${ }^{13}$ C-NMR (101 MHz, $\mathrm{CDCl}_{3}$ ) of compound $\mathbf{2 g}$

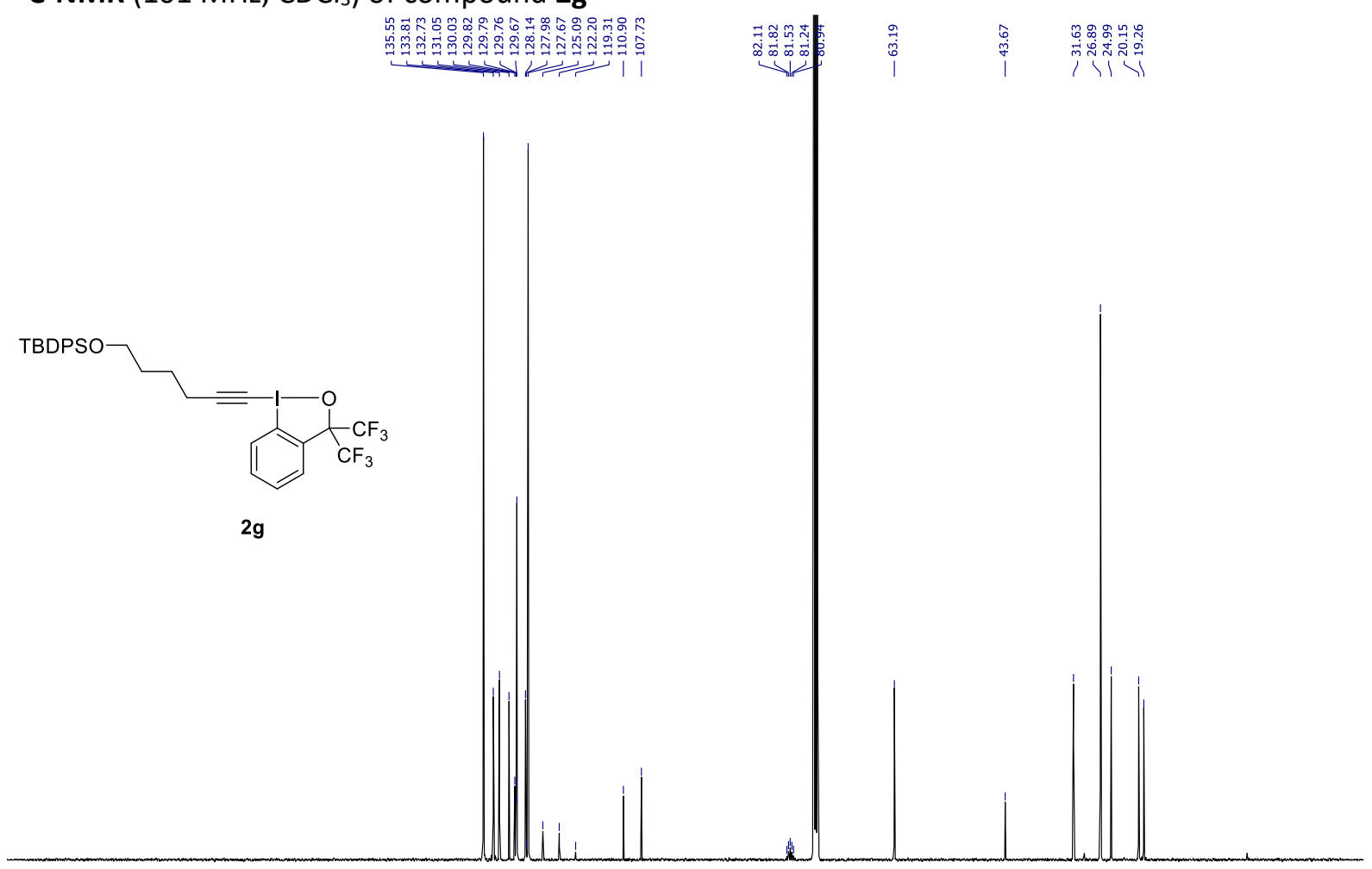

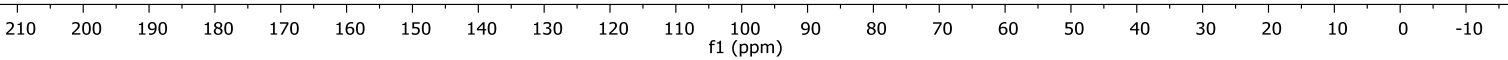


${ }^{19}$ F-NMR (376 MHz, CDCl $)$ of compound $\mathbf{2 g}$

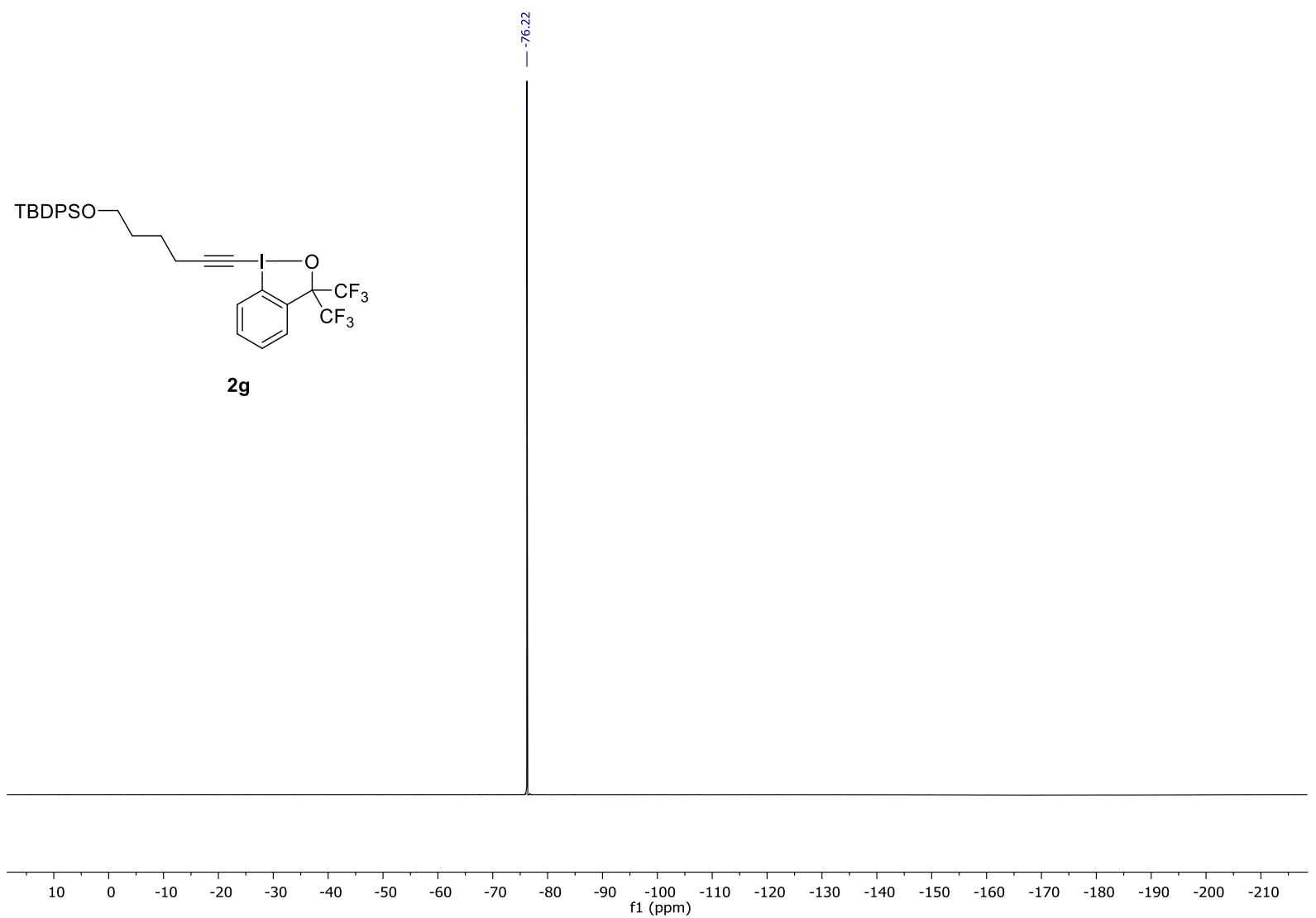




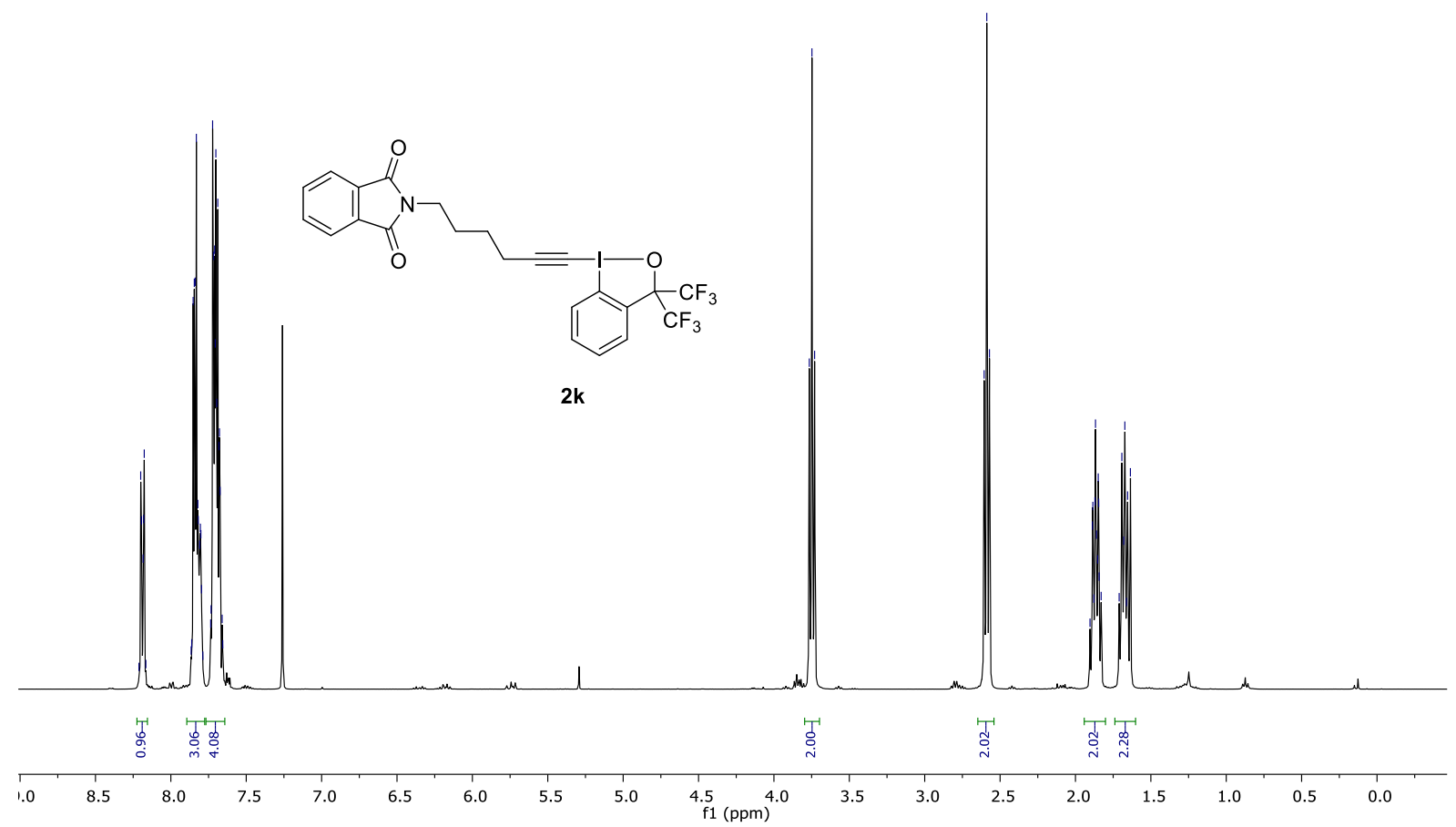

${ }^{13} \mathrm{C}-\mathrm{NMR}\left(101 \mathrm{MHz}, \mathrm{CDCl}_{3}\right)$ of compound $\mathbf{2 k}$

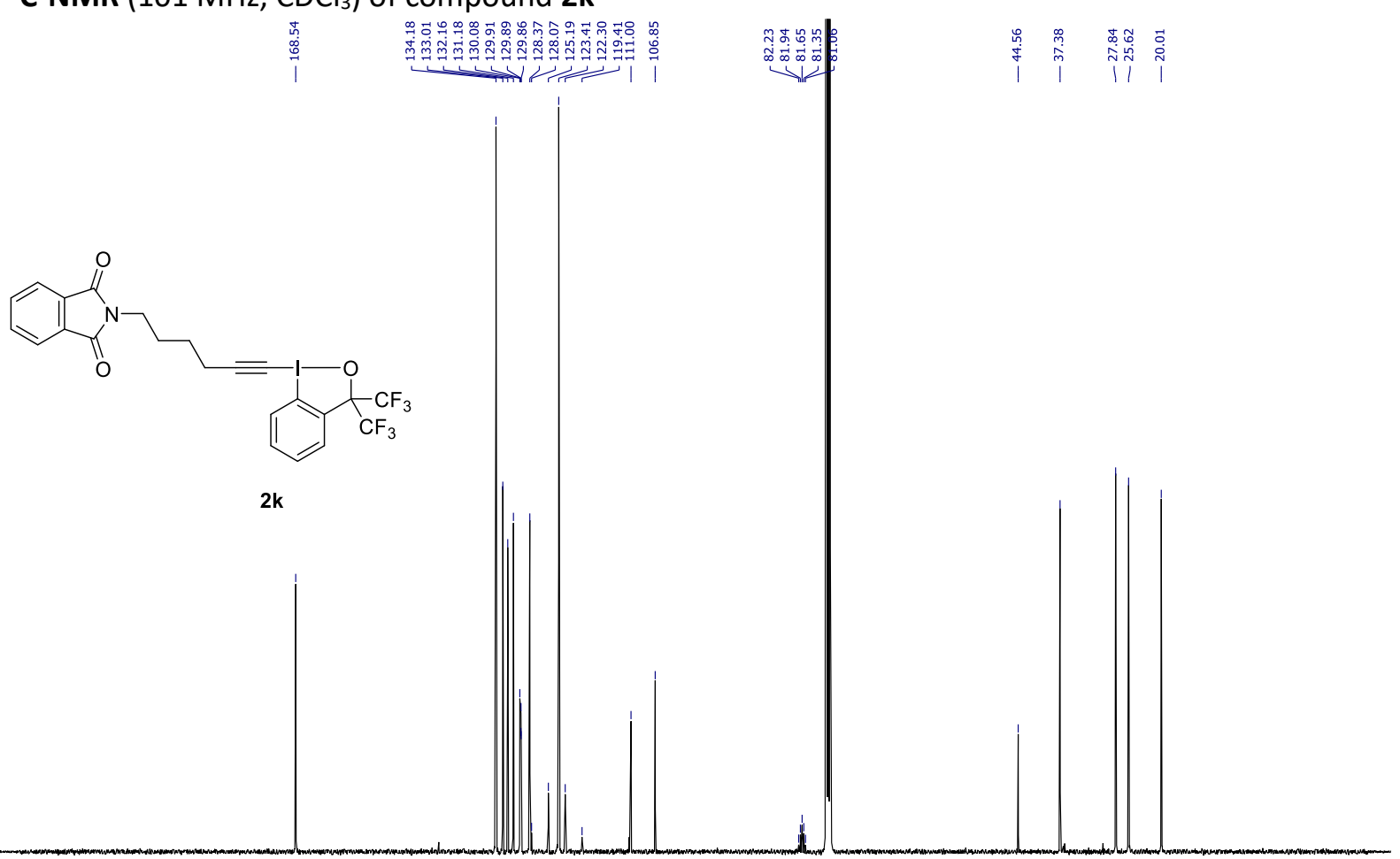

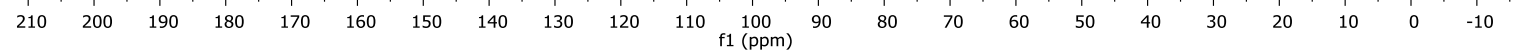


${ }^{19}$ F-NMR (376 MHz, $\mathrm{CDCl}_{3}$ ) of compound $\mathbf{2 k}$

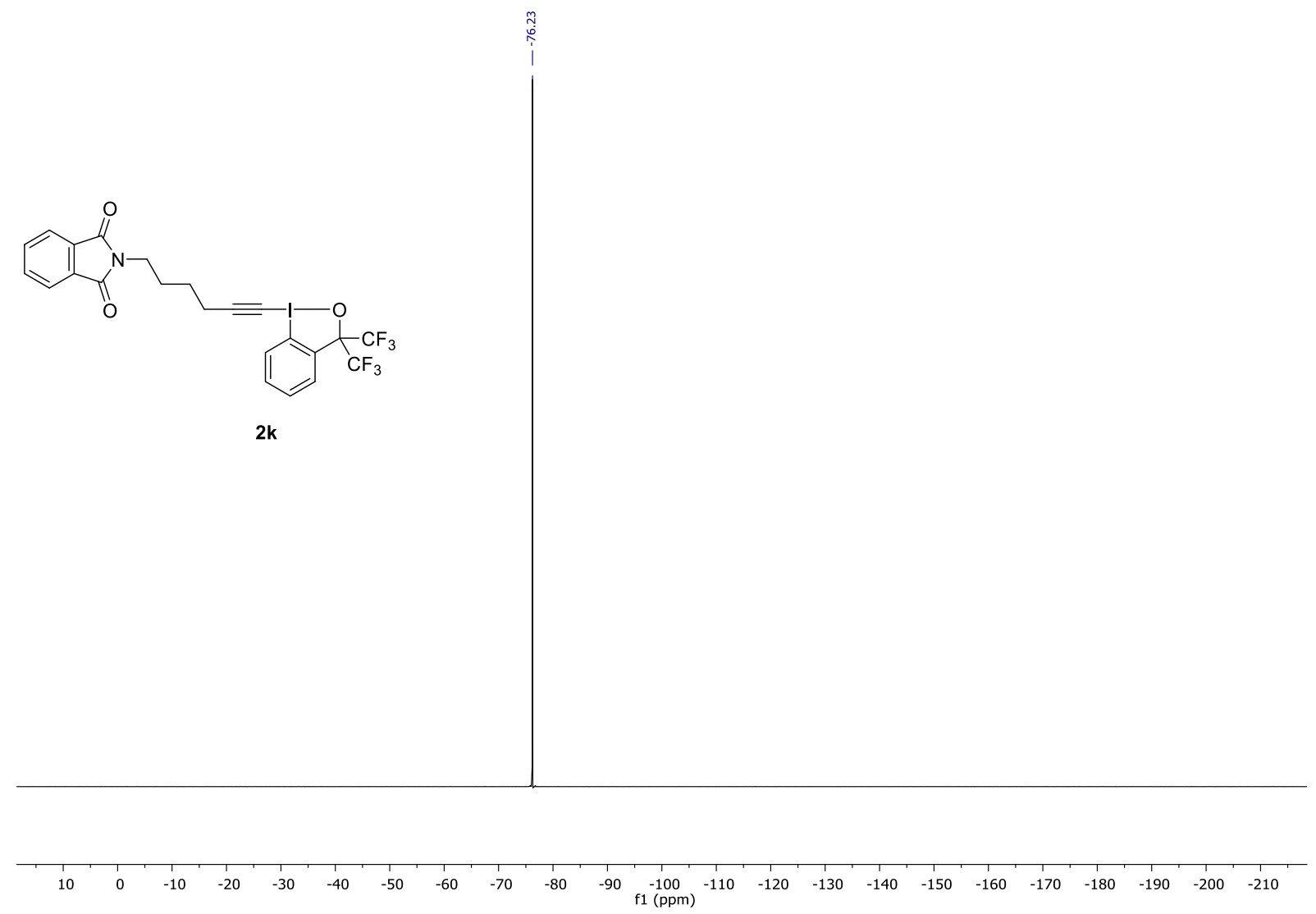


${ }^{1} \mathrm{H}-\mathrm{NMR}\left(400 \mathrm{MHz}, \mathrm{CDCl}_{3}\right)$ of compound 39

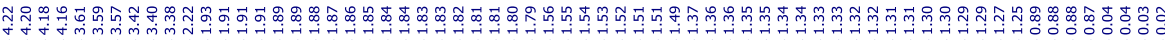<smiles>CCOC(=O)OCCCCC(C(C)=O)C(=O)OCC</smiles>

39

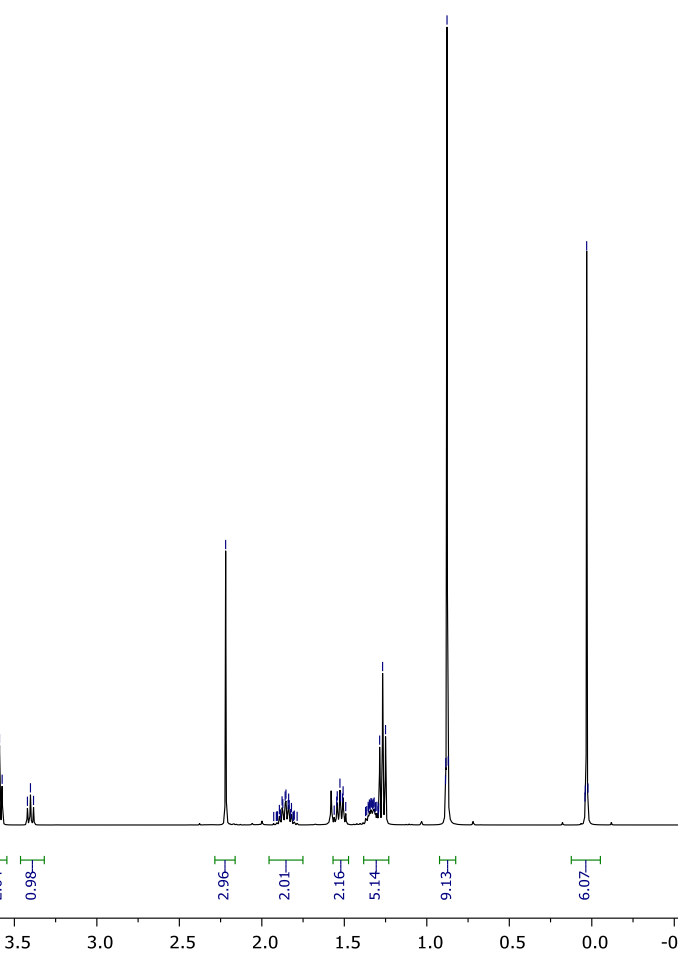

${ }^{13} \mathrm{C}-\mathrm{NMR}\left(101 \mathrm{MHz}, \mathrm{CDCl}_{3}\right.$ ) of compound 39

$$
\text { i }
$$<smiles>CCOCCCCC(C(C)=O)C(=O)OCC</smiles>

39
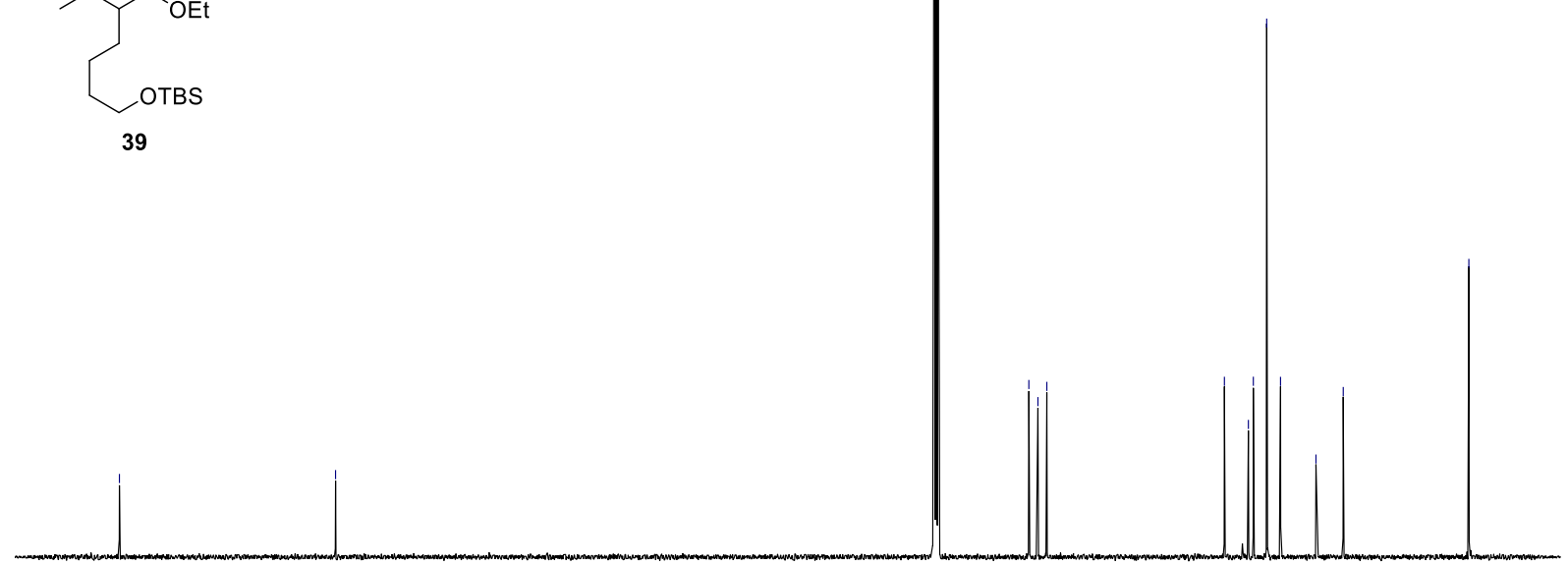
${ }^{1} \mathrm{H}-\mathrm{NMR}$ (400 MHz, $\mathrm{CDCl}_{3}$ ) of compound $\mathbf{4 0}$<smiles>CCOC(=O)C(CCCC(=O)O)C(C)=O</smiles>

40

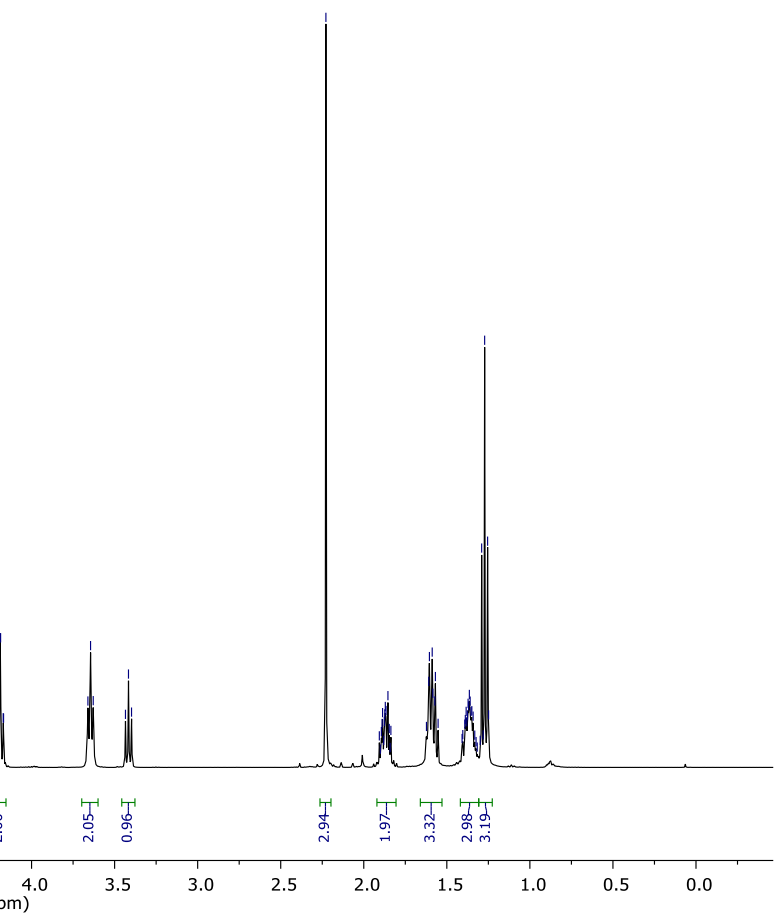

${ }^{13} \mathrm{C}-\mathrm{NMR}\left(101 \mathrm{MHz}, \mathrm{CDCl}_{3}\right)$ of compound 40

I :<smiles>CCOC(=O)C(CCCO)C(C)=O</smiles>

40
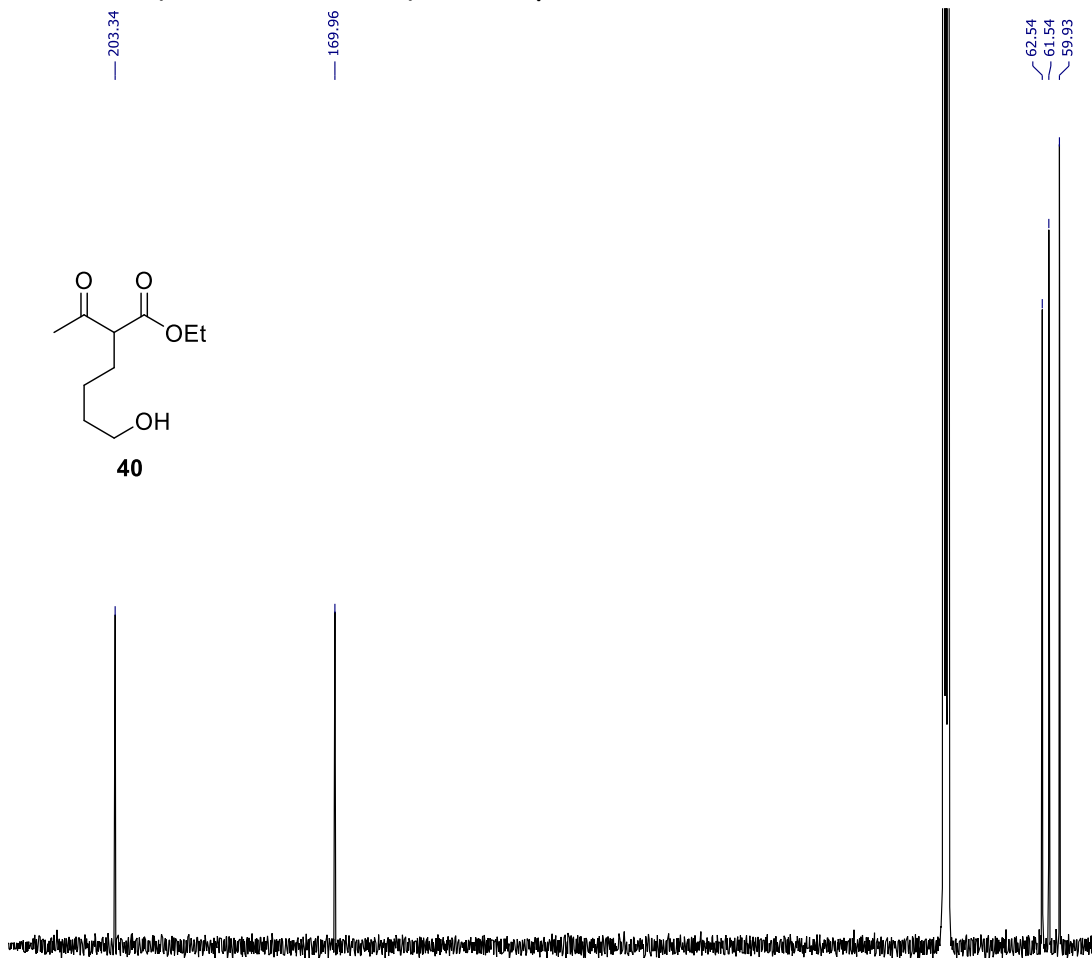

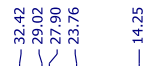

$\begin{array}{llllllllllll}210 & 200 & 190 & 180 & 170 & 160 & 150 & 140 & 130 & 120 & 110 & \begin{array}{l}100 \\ \mathrm{f} 1(\mathrm{ppm})\end{array}\end{array}$ 
${ }^{1} \mathbf{H}-\mathrm{NMR}\left(400 \mathrm{MHz}, \mathrm{CDCl}_{3}\right.$ ) of compound $\mathbf{5 6 k}$

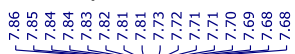
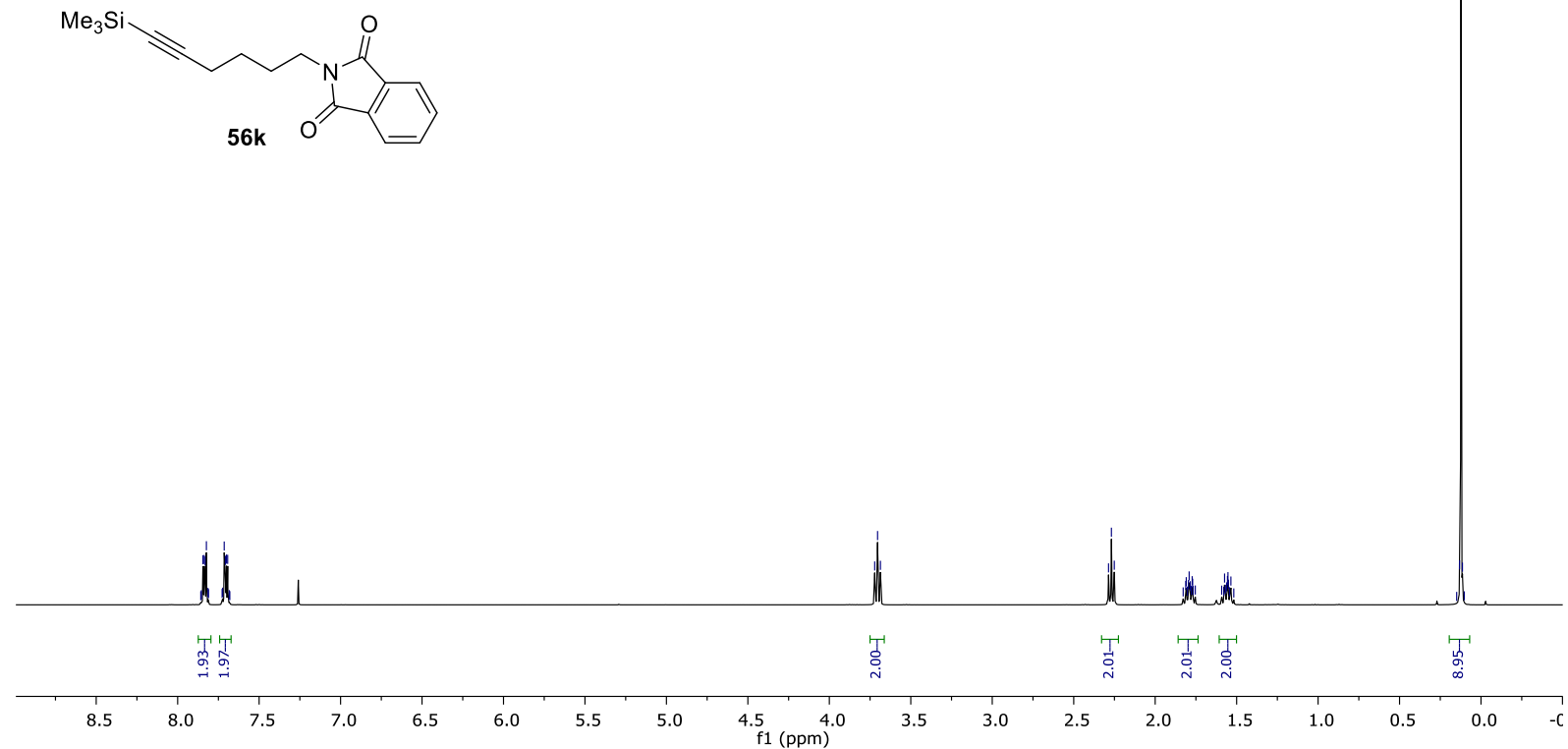

${ }^{13}$ C-NMR (101 MHz, $\mathrm{CDCl}_{3}$ ) of compound 56k

兽
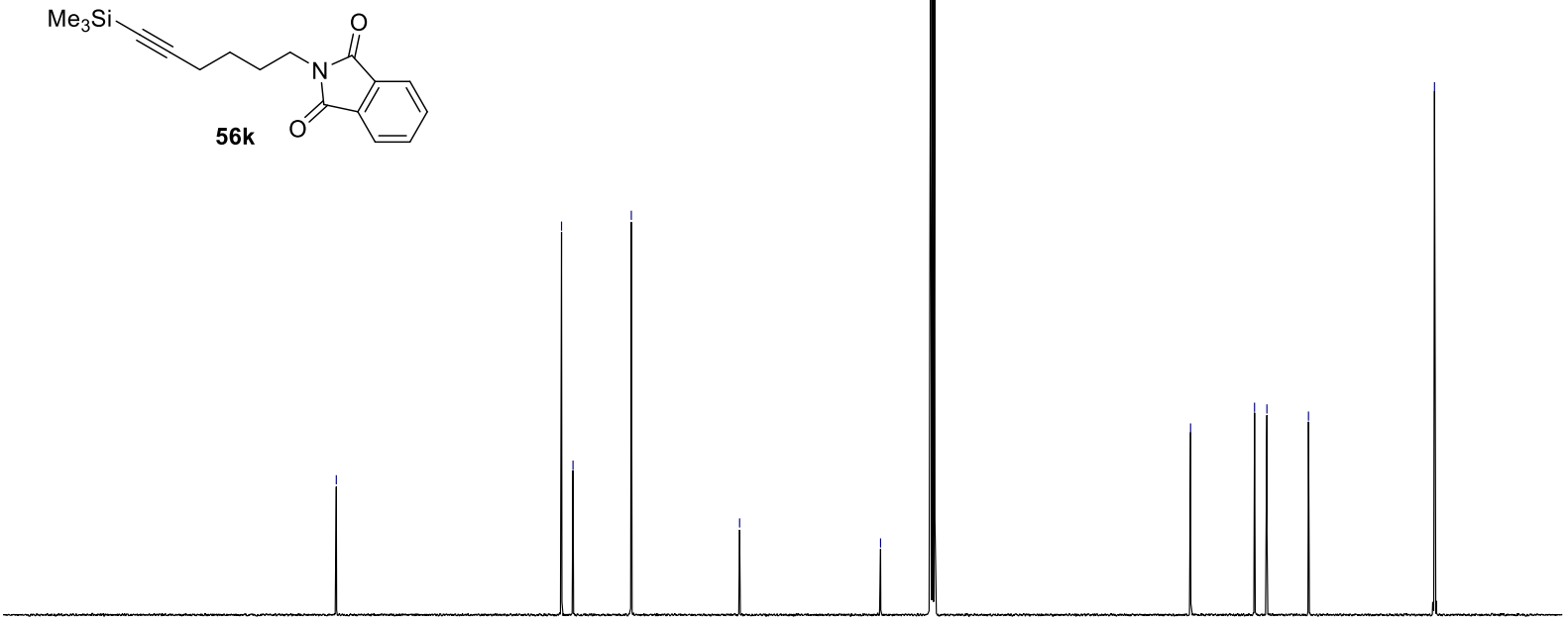

$\begin{array}{lllllllllllllllllllllllllllll}210 & 200 & 190 & 180 & 170 & 160 & 150 & 140 & 130 & 120 & 110 & 100 & 90 & 80 & 70 & 60 & 50 & 40 & 30 & 20 & 10 & 0 & -10\end{array}$ 
8. X-ray diffraction parameters and data for 5 aj
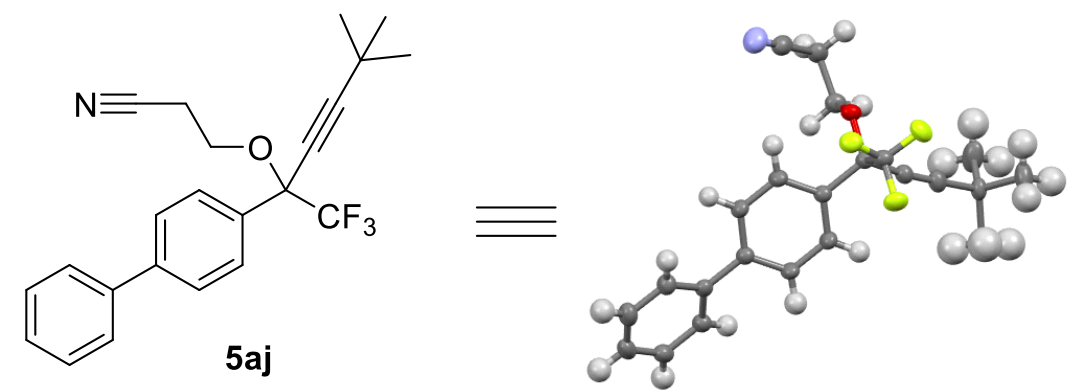

Empirical formula

$\mathrm{C}_{23} \mathrm{H}_{22} \mathrm{~F}_{3} \mathrm{NO}$

Formula weight

385.41

Temperature

$140.00(10) \mathrm{K}$

Wavelength

$1.54184 \AA$

Crystal system

Orthorhombic

Space group

Unit cell dimensions

Pbca

$\begin{array}{ll}\mathrm{a}=8.81133(9) \AA & \alpha=90^{\circ} . \\ \mathrm{b}=20.9653(2) \AA & \beta=90^{\circ} . \\ \mathrm{c}=22.3585(3) \AA & \gamma=90^{\circ} .\end{array}$

Volume

$4130.33(8) \AA^{3}$

Z

8

Density (calculated)

Absorption coefficient

$1.240 \mathrm{Mg} / \mathrm{m}^{3}$

$0.785 \mathrm{~mm}^{-1}$

$\mathrm{F}(000)$

Crystal size

1616

$0.440 \times 0.151 \times 0.120 \mathrm{~mm}^{3}$

$\Theta$ range for data collection

3.954 to $72.795^{\circ}$.

$-10 \leq h \leq 10,-25 \leq k \leq 25,-27 \leq 1 \leq 27$

ndex ranges

32007

$4078\left[R_{\text {int }}=0.0354\right]$

$99.8 \%$

Gaussian

1.000 and 0.548

Max. and min. transmission

Refinement method

Full-matrix least-squares on $\mathrm{F}^{2}$

Data / restraints / parameters

4078 / 133 / 349

Goodness-of-fit on $\mathrm{F}^{2}$

1.053

Final $R$ indices $[I>2 \sigma(I)]$

$R_{1}=0.0308, w R_{2}=0.0838$

$R$ indices (all data)

$R_{1}=0.0374, w R_{2}=0.0864$

Extinction coefficient

$0.00022(6)$

Largest diff. peak and hole

0.275 and -0.189 e. $\AA^{-3}$ 